\author{
Universidade de São Paulo \\ Instituto de Física
}

\title{
Uma proposta experimental para o teletransporte bicolor de estados quânticos da luz
}

\author{
Paula Sampaio Meirelles
}

Orientador: Prof. Dr. Marcelo Martinelli

Tese de doutorado apresentada ao

Instituto de Física para a obtenção do

título de Doutor em Ciências.

(Versão corrigida)

Banca examinadora:

Prof. Dr. Marcelo Martinelli (IFUSP)

Prof. Dr. André Bohomoletz Henriques (IFUSP)

Prof. Dr. Antônio Martins Figueiredo Neto (IFUSP)

Prof. Dr. Celso Jorge Villas Boas (UFSCar)

Prof. Dr. Reinaldo Luiz Cavasso Filho (UFABC)

São Paulo - 2015 
FICHA CATALOGRÁFICA

Preparada pelo Serviço de Biblioteca e Informação do Instituto de Física da Universidade de São Paulo

Meirelles, Paula Sampaio

Uma proposta experimental para o teletransporte bicolor de estados quânticos da luz. São Paulo, 2015.

Tese (Doutorado) - Universidade de São Paulo.

Instituto de Física. Departamento de Física Experimental

Orientador: Prof. Dr. Marcelo Martinelli

Área de Concentração: Ótica

Unitermos: 1. Ótica quântica; 2. Física experimental; 3. Informação quântica.

USP/IF/SBI-110/2015 


\section{Agradecimentos}

Gostaria de agradecer tudo o que aprendi e vivenciei ao longo desses desafiantes anos. Apesar da frustração de não ter realizado o teletransporte quântico, fico imensamente feliz em perceber toda a minha evolução pessoal e espiritual durante essa jornada.

Sou grata à Capes por ter me financiado. Realmente, sinto-me uma privilegiada por ter recebido para aprender tanto e me divertir no laboratório. Que eu seja capaz de retornar cada centavo investido para ajudar o país a crescer;

Sou profundamente grata a todo o pessoal do LMCAL, tanto aos professores, Marcelo e Paulo, quanto aos pós-docs e alunos com os quais tive contato durante esse tempo: Renné, Ashok, Márcio, Alessandro, Katiúscia, Felippe, Antônio, Hans, Carlos, Harold, Klara, Igor, Rayssa, Túlio, Renato, Flávio e Luiz. O convívio com vocês me fez, sem dúvida, uma pessoa melhor e muito mais consciente dos meus propósitos de vida;

Agradeço, em especial, ao Renné, parceiro de experimento que me ensinou muito das práticas de laboratório e me fez perder o medo de manipular um laser intenso. Agradeço também por ter cedido seu código para a realização de algumas das análises de dados, enquanto o meu código não estava finalizado;

Ao professor Piza, pela longa e excelente discussão em sua sala em pleno Carnaval. Foi um dos momentos mais felizes do meu doutorado onde me senti, de fato, ouvida e considerada. Gratidão!

Aos queridos técnicos da mecânica, eletrônica e vácuo com os quais trabalhei: Marcos, Júnior, Pedro, Alex, Betão, Wilson, Eduardo, Celso e João pelo profissionalismo e por todos os ensinamentos. Vocês foram também meus professores em vários momentos, orientando-me em questões práticas que jamais imaginei lidar. Muita gratidão!

Aos funcionários e amigos: Edi, Juliane, Luiz, Sérgio e Pedro (da gráfica) pela paciência, torcida e palavras de conforto sempre que o experimento apresentava algum problema;

À minha mãe amada e exemplo na minha vida, Nevinha, pelo amor e 
apoio incondicional;

À minha irmã Daniela, ao meu cunhado Marcelo e às minhas pequenas Marina e Rafaela pelo carinho e por encher minha vida de felicidade e esperança;

Ao meu pai Paulo por me colocar nesse maravilhoso caminho da busca pelo conhecimento;

À toda minha família pela torcida, especialmente, às minhas tias Zaira, Tânia e Aline pelas orações;

Ao meu primo André por ter financiado quase toda a minha educação durante o ensino fundamental e médio. Sinto-me, mais uma vez, uma privilegiada por ter estudado em boas escolas em Salvador, isso fez toda a diferença na minha formação. Certamente, sem o seu financiamento dificilmente estaria na USP. Minha eterna gratidão!!!!

Aos meus amigos, Mariana, Nataly, Telma, Ghrasy, Fernanda, Néia, Tiago, Ísis, Maria Clara, Juliana, Sérgio, Raimundo, Ângelo e Maicon pela amizade e por me sentir tão querida;

E, o mais importante de todos os agradecimentos, ao DIVINO por me guiar pela escuridão e me dar sabedoria para lidar com situações pantanosas. 


\section{Resumo}

Propomos um esquema inovador para o teletransporte em variáveis contínuas usando um OPO, oscilador paramétrico ótico, como fonte de estados emaranhados e desenvolvemos a instrumentação necessária para implementá-lo. O OPO é utilizado em um regime de operação onde dois feixes de comprimentos de onda distintos são produzidos, o que permitirá que o estado de uma dada cor seja aniquilado e que sua recriação seja em uma cor diferente.

A realização dessa proposta permite teletransportar estados quânticos da luz entre cores distintas do espectro eletromagnético. Devido a sintonizabilidade do OPO, será possível a comunicação quântica entre diferentes tipos de sistemas que servirão como elementos de processamento na manipulação da informação quântica.

A fidelidade deste processo é tanto maior quanto maior for o emaranhamento do par empregado, dessa forma, o maior desafio para a realização do teletransporte é justamente a construção de um canal quântico com o maior nível de emaranhamento possível experimentalmente.

Várias tentativas de construção do canal quântico foram feitas, porém nenhuma delas apresentou-se, até o momento, adequada para a realização com sucesso do protocolo. Em uma das tentativas, um OPO duplamente ressonante, alcançou-se uma compressão de ruído de intensidade, nunca antes medida na história do nosso laboratório, porém o excesso de ruído de fase medido, originado do laser, degradou o emaranhamento, impossibilitando o prosseguimento da montagem.

Atualmente, estamos investindo em um OPO triplamente ressonante como provável canal quântico do nosso protocolo. Medidas preliminares de emaranhamento desse novo OPO foram realizadas, entretanto ainda não se mostrou suficiente para o teletransporte. 



\section{Abstract}

This work has aimed to implement and develop the instrumentation needed in an innovative approach of the teleportation in continuous variables using an OPO, optical parametric oscillator, as source of entangled states. OPO is used in a type of operation where two intense beams of different wavelengths are produced. It allows that the state of a given color is annihilated and reconstructed in a different color.

The realization of the bicolor teleport will make possible the interaction of fields with other entanglement experiments such atoms that operate at different frequencies. Due to the tunable OPO, quantum communication between different types of systems, which serve as processing elements in the manipulation of quantum information, will be possible.

The protocol's fidelity depends on the level of entanglement between the beams so the greatest challenge of our set up is the construction of a quantum channel with the highest level of entanglement experimentally achieved.

Several attempts of quantum channel were constructed, but none of them appeared suitable for carrying out successfully the protocol. In one of the attempts, a doubly resonant OPO reached a intensity noise compression that has never been measured in the history of our laboratory but the excess phase noise measured, originated from the commercial laser, degraded entanglement and prevented the continuation of the set up.

We are working now with a triply resonant OPO as a probable quantum channel of the protocol. Preliminary measurements of entanglement of this new OPO were done, although it still was not enough to implement teleportation. 



\section{Sumário}

$\begin{array}{ll}\text { Agradecimentos } & 3\end{array}$

$\begin{array}{ll}\text { Resumo } & 5\end{array}$

$\begin{array}{ll}\text { Abstract } & 7\end{array}$

1 Introdução 13

2 Conceitos introdutórios em Ótica Quântica 17

2.1 Descrição quântica da luz . . . . . . . . . . . . . . . 17

2.1.1 Estados de Fock e quadraturas do campo . . . . . . . . 18

2.1.2 Estados coerentes . . . . . . . . . . . . . . 20

2.1.3 Estados comprimidos . . . . . . . . . . . . 24

3 Medidas das quadraturas do campo eletromagnético 27

3.1 O fotodector e a geração das fotocorrentes . . . . . . . . . . . 27

3.1.1 Fotodiodo . . . . . . . . . . . . . 28

3.1.2 Eficiência quântica . . . . . . . . . . . . . . . 32

3.1.3 Circuito simplificado do detector . . . . . . . . . 34

3.2 Detecção homodina balanceada . . . . . . . . . . . . . . . 35

3.3 Rotação da elipse de ruído . . . . . . . . . . . . . . . . . . . 42

3.4 A calibração do "shot noise" . . . . . . . . . . . . . . . . 48

3.5 Considerações sobre medidas e teoria de flutuação . . . . . . . 53

3.5.1 Funções de probabilidade dos processos estocásticos . . 54

3.5.2 Processos estocásticos estacionários . . . . . . . . . 57

3.5.3 A densidade espectral do processo e sua medida . . . . 58

3.6 A portadora e as bandas laterais . . . . . . . . . . . . . 60

3.6.1 Modulação de amplitude . . . . . . . . . . . . . 62

3.6.2 Modulação de frequência . . . . . . . . . . . . . . 65

3.7 Cavidades Óticas . . . . . . . . . . . . . . . . . . . 70 
4 Teletransporte Quântico $\quad 77$

4.1 O teorema da não-clonagem . . . . . . . . . . . . . . 77

4.2 O protocolo original proposto . . . . . . . . . . . . . . 80

4.3 A fidelidade do teletransporte . . . . . . . . . . . . 86

4.4 Teletransporte em variáveis discretas . . . . . . . . . . . . 87

4.4.1 O papel do BS na medida de Bell . . . . . . . . . 88

4.4.2 A estação de Bob e os resultados obtidos . . . . . . . . . 90

4.5 Teletransporte em variáveis contínuas . . . . . . . . . . . . . 91

4.5.1 Fonte de estados emaranhados . . . . . . . . . . . . 92

4.5.2 A "medida de Bell" em Alice . . . . . . . . . . . . . 96

4.5.3 A reconstrução do estado em Bob . . . . . . . . . . . . 98

4.5.4 "Quantum duties" e resultados . . . . . . . . . . . . 102

5 Proposta do teletransporte quântico bicolor 107

5.1 Motivação da ideia do teletransporte bicolor . . . . . . . . . . 107

5.2 Visões gerais das propostas . . . . . . . . . . . . . . . . . 109

5.2 .1 Proposta inicial com um DROPO . . . . . . . . . 110

5.2.2 Proposta atual com um TROPO . . . . . . . . 115

6 Montagem experimental e resultados preliminares $\quad 119$

6.1 Caracterização dos detectores . . . . . . . . . . . . . . . 120

6.1.1 Eficiência medida e saturação do detector . . . . . . 120

6.1.2 "Shot noise" e o ruído eletrônico . . . . . . . . . . . . 121

6.2 Sistema de refrigeração e montagem . . . . . . . . . . . . . . . 125

6.2.1 Câmara de vácuo . . . . . . . . . . . . . . . . . 128

6.2.2 Montagem no interior da câmara . . . . . . . . . . . . 129

6.3 O canal clássico EOM e a sua caracterização . . . . . . . . . . 133

6.3.1 Moduladores eletro-óticos . . . . . . . . . . . 135

6.4 Caracterização dos EOMs . . . . . . . . . . . . . . . . 137

6.4.1 Interferômetro de Mach-Zehnder (MZ) . . . . . . . . 138

6.4.2 Interferômetro de MZ com um EOM . . . . . . . . . . 141

6.4.3 Montagem experimental e resultados . . . . . . . . . 144

6.5 Construção do canal quântico: tentativas . . . . . . . . . . . . 148

6.5.1 Considerações gerais sobre um OPO . . . . . . . . 148

6.5.2 Tentativa 1: DROPO1 .............. 153

6.5.3 Tentativa 2: DROPO2 . . . . . . . . . . 154

6.5.4 Cavidade de filtro para o bombeio . . . . . . . . . . 170

6.5.5 Tentativa 3: TROPO1 . . . . . . . . . . . . . . . 172

6.5.6 Tentativa 4: TROPO2 . . . . . . . . . . . 174 
A O papel da eletrônica 181

A.1 Filtros eletrônicos: RC passa-alta, baixa e banda . . . . . . . . 181

A.2 Amplificador operacional e ganho dos circuitos . . . . . . . . . 186

A.3 Função de transferência e gráfico de Bode . . . . . . . . . . . 190

B Amplificação e segunda filtragem do sinal 197

$\begin{array}{ll}\text { Referências Bibliográficas } & 201\end{array}$ 


\section{Capítulo 1}

\section{Introdução}

O emaranhamento é uma forma de correlação intrinsecamente quântica que tem como principal propriedade o fato de que a informação contida no estado global de um dado sistema é maior que a soma das informações contidas em cada uma de suas partes. Devido a essa curiosa propriedade, ele representa o elemento principal na implementação de protocolos que visam transmitir a informação contida em sistemas quânticos, protocolo conhecido como teletransporte, ou mesmo que visam a transmissão mais segura de informação clássica.

Estados emaranhados em sistemas de variáveis contínuas, referente a dimensionalidade infinita do espaço de Hilbert em estudo, foram demonstrados experimentalmente através do uso de Osciladores Paramétricos Óticos (OPO) operando acima do limiar de oscilação $[1,2,3,4]$.

Devido às correlações em fase e em amplitude entre os feixes produzidos, o OPO serve como fonte de estados emaranhados, requisito fundamental para a realização do teletransporte quântico.

O grupo do Laboratório de Manipulação de Átomos e Luz (LMCAL) tem estudado sistematicamente o OPO que, oscilando acima do seu limiar, é capaz de gerar feixes de cores distintas, correlacionados quanticamente em intensidade e fase.

Desde a construção do nosso primeiro OPO [5], importantes resultados foram obtidos como: a obtenção do emaranhamento bipartite dos feixes gêmeos [1], a previsão teórica do emaranhamento tripartite [2], as primeiras medidas de correlação quântica entre os feixes (bombeamento, sinal e complementar) [4], e a obtenção do emaranhamento tripartite [6]. Todos esses resultados abriram a possibilidade para a aplicação do OPO em um esquema de teletransporte de estados quânticos da luz.

Baseado no primeiro trabalho experimental de teletransporte em variáveis contínuas [7], foi proposto um esquema para a implementação desse protocolo 
no LMCAL que utilizará o estado emaranhado produzido por um OPO para realizar o teletransporte entre cores distintas do espectro eletromagnético. Grande parte do trabalho realizado durante o presente doutorado foi desenvolver a instrumentação necessária para a realização desse esquema.

O teletransporte é realizado quando a informação contida em um dado estado desconhecido é transportada, com o auxílio de canais clássicos e quântico, de uma estação, operada por Alice, para outra, operada por Bob. No caso da proposta do LMCAL, o estado a ser teletransportado é produzido em uma estação, conhecida como Victor, através da modulação de fase de um estado coerente e é enviado à estação operada por Alice.

Os feixes gêmeos produzidos pelo OPO são separados por um PBS (divisor polarizante de feixe) e um desses feixes incide numa cavidade ótica, chamada de filtro, para separar o feixe coerente de suas flutuações associadas ao excesso de ruído proviniente do emaranhamento. Esse feixe coerente serve de base para Victor produzir o estado a ser teletransportado. Com o uso de um EOM (modulador eletro-ótico), Victor cria esse estado através da modulação do campo clássico e, assim, introduz flutuações de fase.

Alice recebe as flutuações do sinal refletidas pela cavidade de filtro e o estado preparado por Victor e os interfere em um BS 50 : 50. As quadraturas $x$ e $p$ são medidas e enviadas para Bob, através do canal clássico, que, usando o feixe complementar recebido da fonte, reconstrói o estado. Por fim, Victor verifica o estado teletransportado. O LMCAL utiliza outro método para medir as flutuações de fase dos campos. São utilizadas cavidades óticas, denominadas de cavidades de análise, em que flutuações de fase do campo incidente são transformadas em flutuações de intensidade do feixe refletido pela cavidade [8].

A realização desse projeto permitirá teletransportar estados quânticos da luz entre cores distintas do espectro eletromagnético e tornará possível o uso desse esquema proposto na interação com outros experimentos de emaranhamento de campos e átomos, ou íons, que trabalham com diferentes frequências. Assim, após a conclusão desse experimento será factível, devido a sintonizabilidade do OPO, a comunicação quântica entre diferentes tipos de sistemas.

A tese divide-se em 6 capítulos e está estruturada da seguinte forma: No capítulo 2, encontra-se uma revisão resumida de alguns conceitos fundamentais de Ótica Quântica, como a quantização do campo eletromagnético e a definição de observáveis. No capítulo 3, muita atenção é dada aos métodos de medida, que compreende tanto a teoria que fundamenta essas técnicas quanto a eletrônica envolvida no processo. O objetivo desse extenso capítulo é tornar mais acessível o entendimento desses dispositivos eletrônicos tão imprecindíveis na realização de experimentos em Ótica Quântica. 
No capítulo 4, é analisado o protocolo do teletransporte originalmente proposto e sua implementação experimental em sistemas de variáveis discretas e contínuas. No capítulo 5, são descritas as nossas duas propostas de implementação do teletransporte bicolor usando, inicialmente, um OPO duplamente ressonante e, depois, um triplamente ressonante.

Finalmente, no capítulo 6, é abordada a caracterização dos detectores, a montagem mecânica e o sistema de refrigeração do OPO, a caracterização do canal clássico do protocolo, assim como as quatro tentativas de construção de um canal quântico com o máximo de compressão de ruído realizadas no período do meu doutorado. 


\section{Capítulo 2}

\section{Conceitos introdutórios em Ótica Quântica}

Antes de lançar-se propriamente na questão do teletransporte de estados quânticos de sistemas de variáveis discretas e contínuas, faz-se necessário primeiro identificar e definir exatamente quais propriedades da luz são utilizadas no processo e de que forma elas são acessadas experimentalmente. Nesse sentido, esse capítulo trata, de forma breve e sucinta, da descrição quântica da luz e, o capítulo seguinte, dos métodos para medir observáveis quânticos da luz.

\subsection{Descrição quântica da luz}

Na teoria eletromagnética clássica, a manipulação adequada das equações de Maxwell fornece as equações de onda para os campos elétrico e magnético, cuja solução, no espaço livre, é uma onda eletromagnética se propagando com a velocidade da luz.

Uma solução possível para o campo elétrico da luz é uma onda plana monocromática, descrita matematicamente por:

$$
\mathbf{E}(\mathbf{r}, t)=\left[E(t) e^{i(\mathbf{k} \cdot \mathbf{r}-\omega t)}+E(t)^{*} e^{-i(\mathbf{k} \cdot \mathbf{r}-\omega t)}\right] \hat{\mathbf{e}}
$$

em que $E(t)$ é a amplitude complexa, $\mathbf{k}$ o vetor de onda, $\omega$ a frequência angular da onda e ê o vetor de polarização da onda.

Contudo, do ponto de vista da teoria quântica, demonstra-se $[9,10,11,12]$ que a quantização do campo descrito por (2.1), resulta em um hamiltoniano cuja estrutura algébrica assemelha-se ao hamiltoniano do oscilador harmônico 
quântico:

$$
\hat{H}=\hbar \omega\left(\hat{a}^{\dagger} \hat{a}+\frac{1}{2}\right)
$$

em que $\hat{a}$ e $\hat{a}^{\dagger}$ são os operadores não hermitianos conhecidos como aniquilação e criação que obedecem as relações de comutação:

$$
\left[\hat{a}, \hat{a}^{\dagger}\right]=1 \quad e \quad[\hat{a}, \hat{a}]=\left[\hat{a}^{\dagger}, \hat{a}^{\dagger}\right]=0
$$

Considerado dentro desse panorama quântico, o campo eletromagnético passa, então, a ser entendido como um modo de um oscilador harmônico quântico, com uma frequência própria $\omega$, em que o fóton é interpretado como um pacote de energia elementar do campo de energia $\hbar \omega$, sensível à atuação dos operadores $\hat{a}^{\dagger}$ e $\hat{a}$.

\subsubsection{Estados de Fock e quadraturas do campo}

Os autoestados da hamiltoniana do campo (2.2) são denominados estados de Fock $(|n\rangle)$, ou estados número, também autoestados do operador denominado número $\hat{N}$ igual a:

$$
\hat{N}=\hat{a}^{\dagger} \hat{a}
$$

De maneira similar ao oscilador harmônico quântico, os operadores $\hat{a}$ e $\hat{a}^{\dagger}$ atuam sobre os estados da base $|n\rangle$ de (2.2) de acordo com as seguintes equações:

$$
\begin{gathered}
\hat{a}|n\rangle=\sqrt{n}|n-1\rangle \\
\hat{a}^{\dagger}|n\rangle=\sqrt{n+1}|n+1\rangle
\end{gathered}
$$

Assim, a equação de autovalor para a hamiltoniana (2.2), cujos autoestados são $|n\rangle$ e os autovalores de energia $E_{n}$, é dada por:

$$
\hat{H}|n\rangle=\hbar \omega\left(\hat{a}^{\dagger} \hat{a}+\frac{1}{2}\right)|n\rangle=\hbar \omega\left(n+\frac{1}{2}\right)|n\rangle=E_{n}|n\rangle,
$$

em que $E_{n}$ é o autovalor correspondente ao estado $|n\rangle$. O conjunto $E_{n}$ forma um espectro discreto de autovalores.

No contexto ótico, os operadores $\hat{a}$ e $\hat{a}^{\dagger}$ atuam sobre os vetores da base de (2.2) de forma que, $\hat{a}^{\dagger}$ cria um fóton de energia $\hbar \omega$, e $\hat{a}$ aniquila um fóton.

Apesar de ser difícil de produzir, em termos experimentais, estados em que o número de fótons seja bem determinado, essa base é bastante útil em problemas teóricos por ser uma base ortonomal, além de autoestados da hamiltoniana do campo.

Quando o número de fótons do modo é nulo, o estado é representado por $|0\rangle$ e caracteriza o estado de vácuo. Mesmo na ausência de fótons, (2.7) 
mostra que a energia do campo é $\frac{1}{2} \hbar \omega$, responsável pelo que é comumente denominado de flutuações do vácuo.

Os operadores não hermitianos $\hat{a}$ e $\hat{a}^{\dagger}$ substituem as amplitudes complexas $E(t)$ e $E^{*}(t)$ respectivamente e (2.1) dá lugar ao operador $\hat{E}(t)$ na representação de Heisenberg:

$$
\hat{E}(t)=\hat{a}(t) e^{-i \omega t}+\hat{a}^{\dagger}(t) e^{i \omega t}
$$

Conforme dito anteriormente, os operadores $\hat{a}$ e $\hat{a}^{\dagger}$ obedecem as relações de comutação dadas por (2.3). Porém, no caso, da representação de Heisenberg desses operadores, o comutador $\left[\hat{a}, \hat{a}^{\dagger}\right]$ torna-se igual a:

$$
\left[\hat{a}(t), \hat{a}^{\dagger}(t)\right]=\delta\left(t-t^{\prime}\right)
$$

Nos experimentos de ótica quântica em variáveis contínuas, as grandezas detectadas pelos fotodetectores, e depois medidas, são a intensidade e a fase da luz. Na teoria quântica, essas grandezas são associadas aos operadores quadraturas do campo eletromagnético $\left(\hat{X}_{\phi}, \hat{Y}_{\phi}\right)$, definidos por:

$$
\left\{\begin{array}{l}
\hat{X}_{\phi}=\hat{a}^{\dagger}(t) e^{i \phi}+\hat{a}(t) e^{-i \phi} \\
\hat{Y}_{\phi}=-i\left(\hat{a}(t) e^{-i \phi}-\hat{a}^{\dagger}(t) e^{i \phi}\right)
\end{array}\right.
$$

em que $\phi$ é uma fase que define as quadraturas.

As quadraturas do campo são análogas aos operadores posição $\hat{x}$ e momento $\hat{p}$ e satisfazem as mesmas relações de comutação nas representações de Schrödinger e Heisenberg respectivamente:

$$
\begin{gathered}
{\left[\hat{X}_{\phi}, \hat{Y}_{\phi}\right]=i} \\
{\left[\hat{X}_{\phi}(t), \hat{Y}_{\phi}\left(t^{\prime}\right)\right]=i \delta\left(t-t^{\prime}\right)}
\end{gathered}
$$

Caso as matrizes que representam esses tipos de operadores apresentem um espectro contínuo de autovalores reais, associados às medidas propriamente, o sistema que elas descrevem é dito ser de variáveis contínuas.

Tendo em vista as equações (2.5) e (2.6), calcula-se a média e a variância das quadraturas do campo sobre os estados de Fock:

$$
\begin{gathered}
\left\langle n\left|\hat{X}_{\phi}\right| n\right\rangle=\left\langle n\left|\hat{Y}_{\phi}\right| n\right\rangle=0 \\
\left\langle n\left|\Delta^{2} \hat{X}_{\phi}\right| n\right\rangle=\left\langle n\left|\hat{X}_{\phi}^{2}\right| n\right\rangle-\left(\left\langle n\left|\hat{X}_{\phi}\right| n\right\rangle\right)^{2}=\left\langle n\left|\Delta^{2} \hat{Y}_{\phi}\right| n\right\rangle=2 n+1
\end{gathered}
$$


Isso significa que, para estados quânticos com número bem determinado de fótons (estados número), a média e a variância (ou incerteza) de todas as quadraturas do campo são iguais e a variância depende linearmente do número de fótons presentes no estado.

Se o estado em questão for o estado de vácuo $|0\rangle$, as incertezas em ambas as quadraturas serão mínimas e:

$$
\left\langle 0\left|\Delta^{2} \hat{X}_{\phi}\right| 0\right\rangle=\left\langle 0\left|\Delta^{2} \hat{Y}_{\phi}\right| 0\right\rangle=1
$$

\subsubsection{Estados coerentes}

Foi Schrödinger quem primeiro introduziu os estados quânticos de mínima incerteza aplicados ao estudo do oscilador harmônico quântico. Porém, foi somente mais tarde, com os trabalhos de Glauber [13], que tais estados foram aplicados ao campo eletromagnético e denominados de estados coerentes. Por serem estados com menor incerteza associada, são os estados quânticos que mais se parecem com os estados clássicos.

Os estados coerentes, denotados por $|\alpha\rangle$, são definidos como auto estados do operador aniquilação $\hat{a}$ e formam uma base contínua do espaço de Hilbert:

$$
\hat{a}|\alpha\rangle=\alpha|\alpha\rangle,
$$

em que $\alpha$ é um número complexo, escrito na forma polar como:

$$
\alpha=|\alpha| e^{i \phi}
$$

É interessante escrever $|\alpha\rangle$ na base de estados de Fock:

$$
|\alpha\rangle=\sum_{n=0}^{\infty} c_{n}|n\rangle,
$$

em que $c_{n}=\langle n \mid \alpha\rangle$.

Atuando-se o operador aniquilação $\hat{a}$ sobre o seu auto estado $|\alpha\rangle$ :

$$
\hat{a}|\alpha\rangle=\sum_{n=0}^{\infty} c_{n} \hat{a}|n\rangle
$$

Através da utilização da equação (2.5), é obtida a seguinte equação:

$$
\hat{a}|\alpha\rangle=\sum_{n=1}^{\infty} c_{n} \sqrt{n}|n-1\rangle
$$


Da igualdade das equações (2.18) e (2.19) aplicadas repetidas vezes, é obtida a seguinte relação para o coeficiente $c_{n}$ :

$$
c_{n}=\frac{\alpha^{n} c_{0}}{\sqrt{n !}}
$$

Assim, reescreve-se (2.17) como:

$$
|\alpha\rangle=c_{0} \sum_{n=0}^{\infty} \frac{\alpha^{n}}{\sqrt{n !}}|n\rangle,
$$

e o bra associado:

$$
\langle\alpha|=c_{0}^{*} \sum_{n=0}^{\infty} \frac{\left(\alpha^{n}\right)^{*}}{\sqrt{n !}}\langle n|
$$

O coeficiente $c_{0}$ é calculado a partir da condição de normalização do estado coerente:

$$
\langle\alpha \mid \alpha\rangle=1
$$

Dessa forma, dada a condição (2.23), o produto escalar de (2.22) e (2.21) é:

$$
\left|c_{0}\right|^{2} \sum_{n=0}^{\infty} \sum_{n^{\prime}=0}^{\infty} \frac{\left(\alpha^{n}\right)^{*} \alpha^{n^{\prime}}}{\sqrt{n !} \sqrt{n^{\prime} !}}\left\langle n \mid n^{\prime}\right\rangle=\left|c_{0}\right|^{2} \sum_{n=0}^{\infty} \frac{|\alpha|^{2 n}}{n !}
$$

Como é sabido, a expansão em série da função exponencial complexa é dada por:

$$
e^{x}=\sum_{n=0}^{\infty} \frac{x^{n}}{n !}
$$

Identificando-se (2.25) em (2.24) e substituindo o valor encontrado para $c_{0}$ em (2.21), encontra-se a expressão para os estados coerentes normalizados:

$$
|\alpha\rangle=\exp \left(-\frac{1}{2}|\alpha|^{2}\right) \sum_{n=0}^{\infty} \frac{\alpha^{n}}{\sqrt{n !}}|n\rangle
$$

Dada a representação do estado coerente $|\alpha\rangle$ na base discreta de Fock em (2.26), percebe-se que o estado coerente não possui um número de fótons bem determinado. Essa característica, aliada ao fato dos estados coerentes serem os estados quânticos mais próximos dos clássicos, torna a base de estados coerentes mais conveniente, do ponto de vista experimental, de ser usada do que a base de estados de Fock. 
É possível calcular a probabilidade $P_{n}$ de detectar $n$ fótons do campo projetando o estado coerente (2.26) no bra $\langle n|$ :

$$
P_{n}=|\langle n \mid \alpha\rangle|^{2}=e^{-|\alpha|^{2}} \frac{|\alpha|^{2 n}}{n !}
$$

A distribuição de probabilidade (2.27) obtida é do tipo Poisson, cuja média é $|\alpha|^{2}$, e demonstra que a intensidade do campo flutua de acordo com a variância dessa distribuição. Isso significa que um detector não percebe um fluxo contínuo e regular de fótons, mas um fluxo aleatório, conhecido como shot noise ou ruído quântico padrão. Por ser um ruído próprio da natureza corpuscular da luz, ele sempre estará presente na detecção do estado coerente, e é normalmente usado como referência nas medidas de ruído de estados quânticos.

Semelhante ao que foi feito anteriormente com os estados de Fock, calculase a média e a variância de $\hat{X}_{\phi}$ e $\hat{Y}_{\phi}$ para o estado coerente $|\alpha\rangle$, dada a equação (2.15):

$$
\begin{gathered}
\left\langle\alpha\left|\hat{X}_{\phi}\right| \alpha\right\rangle=\alpha+\alpha^{*} \quad \text { e } \quad\left\langle\alpha\left|\hat{Y}_{\phi}\right| \alpha\right\rangle=-i\left(\alpha-\alpha^{*}\right) \\
\left\langle\alpha\left|\Delta^{2} \hat{X}_{\phi}\right| \alpha\right\rangle=\left\langle\alpha\left|\Delta^{2} \hat{Y}_{\phi}\right| \alpha\right\rangle=1
\end{gathered}
$$

Observa-se de (2.29) e (2.14) que as incertezas das quadraturas do campo elétrico para o estado coerente são iguais e as mesmas do estado de vácuo, mostrando que o estado coerente também é um estado de incerteza mínima. Como o estado de vácuo $|0\rangle$ também é um estado coerente, em que $\alpha=0$, e qualquer estado coerente $|\alpha\rangle$ tem as mesmas incertezas nas quadraturas do campo, então pode-se concluir que as flutuações quânticas de um estado coerente originam-se das flutuações do vácuo [13].

Como os operadores quadratura $\hat{X}_{\phi}$ e $\hat{Y}_{\phi}$ são observáveis, então eles obedecem a relação de incerteza ${ }^{1}$

$$
\left\langle\Delta^{2} \hat{X}_{\phi}\right\rangle\left\langle\Delta^{2} \hat{Y}_{\phi}\right\rangle \geq\left|\left\langle\left[\hat{X}_{\phi}, \hat{Y}_{\phi}\right]\right\rangle\right|^{2}
$$

Do comutador (2.10), resulta que a relação de incerteza entre as quadraturas do campo é dada pela seguinte inequação:

$$
\left\langle\Delta^{2} \hat{X}_{\phi}\right\rangle\left\langle\Delta^{2} \hat{Y}_{\phi}\right\rangle \geq 1
$$

\footnotetext{
${ }^{1} \mathrm{~A}$ relação de incerteza é dada por: dados dois observáveis $\hat{A}$ e $\hat{B}$ quaisquer, então para qualquer estado, eles obedecem a desigualdade $\left\langle\Delta^{2} \hat{A}\right\rangle\left\langle\Delta^{2} \hat{B}\right\rangle \geq \frac{1}{4}|\langle[\hat{A}, \hat{B}]\rangle|^{2}$. O fator $\frac{1}{4}$ foi omitido, visto que os operadores quadratura do campo podem ser definidos como $\hat{X}_{\phi}=\frac{1}{2}\left(\hat{a}+\hat{a}^{\dagger}\right)$ e $\hat{Y}_{\phi}=\frac{1}{2 i}\left(\hat{a}-\hat{a}^{\dagger}\right)$. O fator $\frac{1}{2}$ não foi levado em consideração nas definições desses operadores apenas para facilitar os cálculos.
} 
Como a variância de um operador diz respeito à incerteza sobre o conhecimento preciso da sua medida, a (2.31) mostra que é impossível saber precisamente e ao mesmo tempo a intensidade e a fase do campo de um dado estado quântico da luz.

Assim, as medidas da fase e da intensidade da luz são correlacionadas e, por causa da limitação física dada por (2.31), menos flutuações na medida em uma delas implica necessariamente no aumento das flutuações na outra. Ver-se-á mais adiante que estados desse tipo são ditos estados comprimidos da luz.

De forma geral, a visão clássica da luz, expressa na equação (2.1), de que ela é uma onda eletromagnética com fase e amplitude bem determinadas, é substituída pela visão quântica que demonstra que tais grandezas são sujeitas às flutuações intrínsecas à própria natureza da luz.

Uma forma ilustrativa de visualizar as incertezas das quadraturas $\hat{X}_{\phi}$ e $\hat{Y}_{\phi}$ para os estados de vácuo e estados coerente é representá-los em um espaço de fase complexo, mais conhecido como diagrama de Fresnel, em que as quadraturas são colocadas nos eixos.
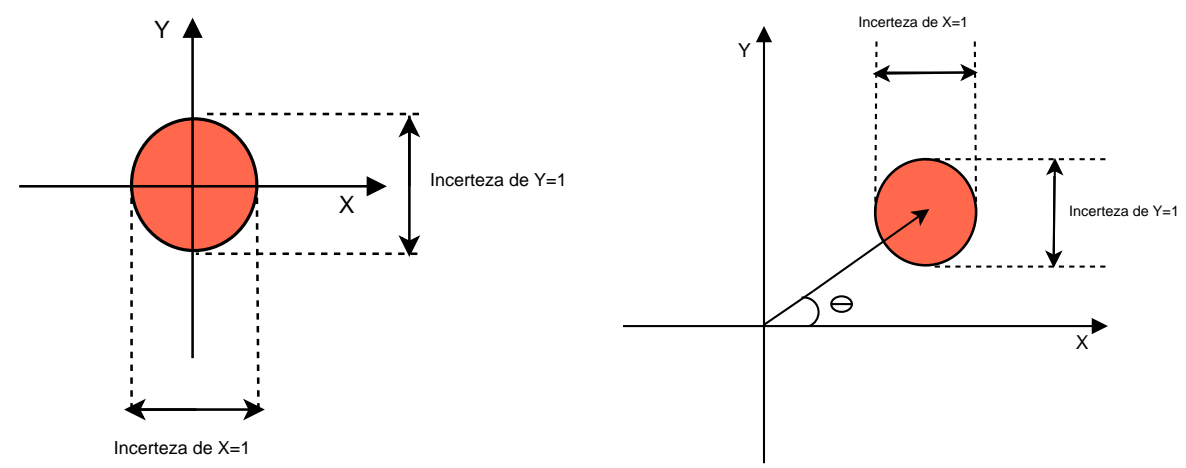

Figura 2.1: Representação no diagrama de Fresnel dos estados de vácuo e coerente, respectivamente. Ambos os estados são estados de incerteza mínima. O estado de vácuo deslocado no diagrama corresponde ao estado coerente.

Os diagramas ilustram que as incertezas das quadraturas são igualmente distribuídas nas direções $\hat{X}$ e $\hat{Y}$ para os estados de vácuo e coerente. Além disso, como as incertezas do estado coerente são as mesmas do estado de vácuo, os estados coerentes são considerados como estados de vácuo deslocados da origem do espaço de fase de $|\alpha|$, associado à amplitude do campo elétrico (2.1).

Outra forma de definir os estados coerentes refere-se justamente à esse deslocamento do estado de vácuo no espaço de fase que é realizado através da atuação de um operador $\hat{D}(\alpha)$, denominado deslocamento, definido como: 


$$
\hat{D}(\alpha)=e^{\alpha \hat{a}^{\dagger}-\alpha^{*} \hat{a}}
$$

É possível mostrar $[10,12]$ que a atuação do operador $\hat{D}(\alpha)$, definido em (2.32), sobre o estado de vácuo, de fato, produz o estado coerente $|\alpha\rangle$ :

$$
|\alpha\rangle=\hat{D}(\alpha)|0\rangle
$$

O estado quântico da luz produzida por um laser, por exemplo, é um estado formado a partir do deslocamento do vácuo no espaço de fase [14], por isso ela é dita ser coerente e se assemelhar aos estados ditos clássicos.

Uma propriedade muito relevante do operador deslocamento é que ele é um operador unitário:

$$
\hat{D}(\alpha) \hat{D}^{\dagger}(\alpha)=\hat{D}^{\dagger}(\alpha) \hat{D}(\alpha)=1
$$

\subsubsection{Estados comprimidos}

Um estado do campo é dito comprimido nas quadraturas $\hat{X}_{\phi}$ e $\hat{Y}_{\phi}$, quando uma delas apresenta uma redução no seu valor, abaixo do valor limite 1, dado em (2.31):

$$
\left\langle\Delta^{2} \hat{X}_{\phi}\right\rangle<1 \quad \text { ou } \quad\left\langle\Delta^{2} \hat{Y}_{\phi}\right\rangle<1
$$

Devido a (2.29), não é possível que as duas quadraturas estejam comprimidas ao mesmo tempo. A figura 2.2 representa um exemplo de estado comprimido no espaço de fases: o vácuo comprimido.

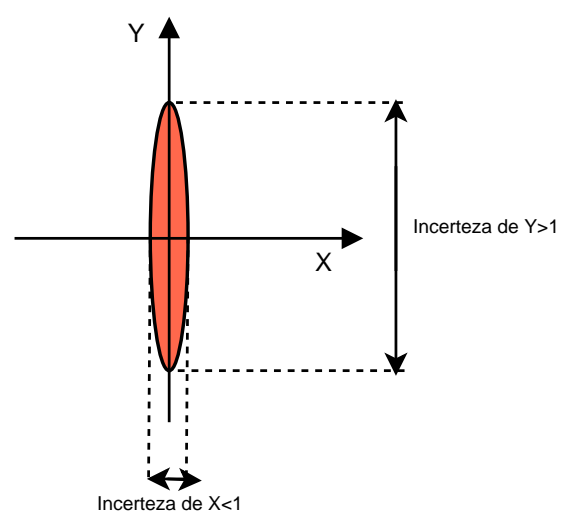

Figura 2.2: Representação no diagrama de Fresnel do estado de vácuo comprimido $|\gamma\rangle$ na quadratura $X_{\phi}$ quando $\phi=0$.

Estados comprimidos são gerados através da atuação de um operador unitário, chamado de operador de compressâo $\hat{S}(\gamma)$, semelhante ao operador 
deslocamento. O operador $\hat{S}(\gamma)$ corresponde a ocorrência do processo físico responsável pela compressão do estado e é definido por:

$$
\hat{S}(\gamma)=e^{\frac{1}{2}\left(\gamma^{*} \hat{a}^{2}-\gamma \hat{a}^{\dagger 2}\right)},
$$

em que $\gamma=r e^{i \phi}$. O parâmetro $r$ é conhecido como parâmetro de compressão e indica o quão comprimida encontra-se a quadratura de um dado estado. Ele pode assumir valores que variam de 0 a infinito.

A atuação do operador $\hat{S}(\gamma)$ em um dado vetor $|\psi\rangle$ de estado, resulta em vetor no vetor de estado comprimido $\left.\psi^{\prime}\right\rangle$ :

$$
\left|\psi^{\prime}\right\rangle=\hat{S}(\gamma)|\psi\rangle
$$

Para o calcular as variâncias dos operadores quadraturas, é necessario o uso das seguintes relações, obtidas com a aplicação do Lemma de Baker-Hausdorf [10]:

$$
\begin{gathered}
\hat{S}^{\dagger}(\gamma) \hat{a} \hat{S}(\gamma)=\hat{a} \cosh (r)-\hat{a}^{\dagger} e^{i \phi} \sinh (r) \\
\hat{S}^{\dagger}(\gamma) \hat{a}^{\dagger} \hat{S}(\gamma)=\hat{a}^{\dagger} \cosh (r)-\hat{a}^{\dagger} e^{-i \phi} \sinh (r)
\end{gathered}
$$

O estado de vácuo comprimido, representado na figura 2.2, é dado por:

$$
|\gamma\rangle=\hat{S}(\gamma)|0\rangle
$$

Usando as equações (2.38), (2.39) e (2.40), é possível calcular as variâncias das quadraturas para o estado de vácuo comprimido [10]:

$$
\begin{aligned}
& \left\langle\gamma\left|\Delta^{2} \hat{X}\right| \gamma\right\rangle=\left[\cosh ^{2}(r)+\sinh ^{2}(r)-2 \sinh (r) \cosh (r) \cos (\phi)\right] \\
& \left\langle\gamma\left|\Delta^{2} \hat{Y}\right| \gamma\right\rangle=\left[\cosh ^{2}(r)+\sinh ^{2}(r)+2 \sinh (r) \cosh (r) \cos (\phi)\right]
\end{aligned}
$$

Para $\phi=0$ nas equações (2.41) e (2.42), observa-se que ocorre a compressão na quadratura $\hat{X}$ e, para $\phi=\pi$, a compressão na quadratura $\hat{Y}$ :

$$
\begin{aligned}
& \phi=0\left\{\begin{array}{l}
\left\langle\Delta^{2} \hat{X}\right\rangle=e^{-2 r} \\
\left\langle\Delta^{2} \hat{Y}\right\rangle=e^{2 r}
\end{array}\right. \\
& \phi=\pi\left\{\begin{array}{l}
\left\langle\Delta^{2} \hat{X}\right\rangle=e^{2 r} \\
\left\langle\Delta^{2} \hat{Y}\right\rangle=e^{-2 r}
\end{array}\right.
\end{aligned}
$$




\section{Capítulo 3}

\section{Medidas das quadraturas do campo eletromagnético}

Esse é o maior capítulo da tese visto a sua enorme importância na compreensão tanto da teoria que envolve as medidas quanto de questões de ordem prática ou experimental. Nele abordaremos o processo de medida de fase e amplitude da luz. Falaremos inicialmente sobre o detector de luz e a geração das fotocorrentes que permite o acesso à informação quântica contida na luz detectada. Além disso, analisaremos a questão do ponto de vista quântico e introduziremos duas técnicas de detecção das quadraturas do campo, associadas a fase e amplitude: a detecção homodina e a auto-homodina.

\subsection{O fotodector e a geração das fotocorren- tes}

A detecção da luz desempenha um papel crucial em ótica quântica experimental, visto que é a base de diversas medidas que contém a informação quântica presente no feixe detectado.

A depender do tipo de experimento realizado, pode-se empregar dois tipos bem distintos de detectores: ou os detectores de fótons individuais ou os detectores que medem correntes elétricas.

Detectores de fótons individuais, usados em experimentos de variáveis discretas (V.D), operam em regime de baixo fluxo de fótons de forma a detectar eventos individuais, conhecidos como "clicks". Já os detectores usados em experimentos de variáveis contínuas (V.C), como o nosso experimento, operam de forma oposta e são capazes de gerar correntes elétricas adquiridas em uma dada janela temporal.

Os detectores que usamos no laboratório são formados pelos chamados 
fotodiodos que utilizam o efeito fotoelétrico para produzir essas correntes elétricas, conhecidas como fotocorrentes. Através da análise do ruído das fotocorrentes é que conseguimos acessar experimentalmente as medidas das quadraturas amplitude e fase do campo detectado.

Para exercer satisfatoriamente esse papel, o fotodiodo responsável pela detecção da luz não-clássica deve possuir, conforme veremos mais adiante, certas características específicas como ser altamente eficiente e ter baixo ruído eletrônico [18].

Faremos, nessa seção, uma descrição estritamente qualitativa dos fotodiodos para entender de que forma essas fotocorrentes são formadas e como elas estão conectadas aos fótons do campo incidente. Em seguida, discutiremos como a fotocorrente gerada é amplificada eletronicamente e separada em sinais ditos $D C$ (Direct Current) e $H F$ (High Frequency).

\subsubsection{Fotodiodo}

Os fotodiodos são componentes eletrônicos da mesma família dos diodos, feitos da junção de regiões com diferentes tipos de dopagens ${ }^{1}$ de um mesmo semicondutor, conhecidos como do tipo $P$ e $N$. Em linhas gerais, a dopagem do material provoca a presença de elétrons livres na banda de condução, no caso $N$, ou a presença de regiões de vacância para elétrons, no caso $P$, conhecidos como "buracos".

Nos diodos, a junção dessas regiões de diferentes dopagens, chamada de junção $P N$, provoca uma polarização inicial de cargas. Na junção $P N$, alguns elétrons livres do material dopado do tipo $N$ difundem para o material dopado do tipo $P$, produzindo na junção uma região de cargas positivas, do lado $N$, e negativas, do lado $P$. Essa região polarizada, conhecida como região de depleção, cria um campo elétrico que impede a passagem de mais elétrons livres de $N$ para $P$. A figura 3.2 ilustra a junção $P N$ e a formação da região de depleção.

Ao aplicar-se uma tensão elétrica externa à junção de tal forma que o material $N$ seja ligado ao pólo positivo e o material $P$ ao negativo, essa barreira aumenta e não há passagem considerável de corrente elétrica no diodo. Na verdade, existe a presença de uma corrente muito pequena, conhecida como corrente de fuga, da ordem de $\mu A$.

\footnotetext{
${ }^{1}$ Os semicondutores são materiais que apresentam uma condutividade elétrica intermediária entre isolantes e condutores. Através do processo químico de dopagem, introduzse controladamente átomos de outros elementos de forma a alterar sua condutividade. A depender da quantidade de elétrons em falta ou em excesso na camada de valência dos átomos introduzidos durante a dopagem, o material é dito do tipo $N$ ou $P$.
} 


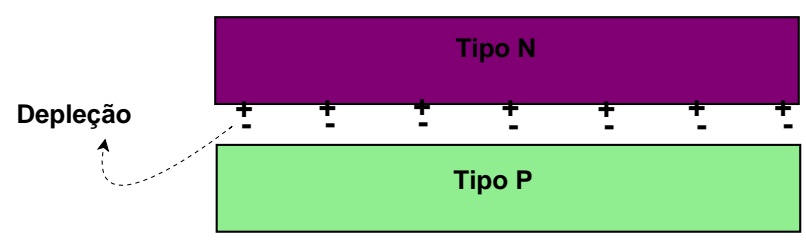

Figura 3.1: Representação de uma junção PN. Em roxo, é visualizado o material semicondutor dopado do tipo $N$ com excesso de elétrons. Em verde, o material do tipo $P$ com excesso de "buracos". Durante o processo de construção da junção, há a migração de elétrons e a formação de uma região polarizada conhecida como depleção. Essa região cria um bloqueio elétrico à passagem de mais eletróns através da junção.

Quando não há passagem de corrente através da junção, diz-se que o diodo está reversamente polarizado. Entretanto, se a tensão externa for invertida tal que o o material $N$ seja ligado ao pólo negativo e o $P$ ao positivo, há uma diminuição dessa barreira elétrica até que, atingido um certo valor, ocorre a passagem de corrente através do diodo e ele é dito polarizado diretamente. A Figura 3.2 ilustra, na parte (a), a representação gráfica do diodo e as suas duas formas de polarização.

Na literatura eletrônica existe uma curva muito conhecida para os diodos, denominada curva característica, que descreve o comportamento da corrente elétrica, $i$, que atravessa um diodo como função da tensão externa aplicada $V_{\text {ext }}$. Essa curva também é vista na figura 3.2 e veremos, mais adiante, que ela se assemelha à curva caracterítica do fotodiodo.

O fotodiodo é um diodo sensível à luz que opera polarizado reversamente e converte a radiação em um sinal elétrico, ou seja, em uma fotocorrente. Em geral, ele é feito de um único material semicondutor dopado de maneira diferente nos lados opostos( de um lado $N$ e do outro $P$ ), e de um material intrínseco $I$, usado na região da junção $P N$, para aumentar a sua resposta espectral [20]. Esse tipo de fotodiodo é conhecido comercialmente como do tipo PIN e é o tipo usado no nosso experimento.

Ao incidir-se fótons sobre o material fotosensível $I$ do fotodiodo, são produzidos, na região de depleção, pares de elétrons-buracos através da interação dos fótons com os átomos do material de $I$, em um processo conhecido como efeito fotoelétrico interno [18]. Com a tensão externa aplicada ao fotodiodo reversamente polarizado, os pares vencem a barreira na região de depleção da junção $P N$ e são acelerados formando uma fotocorrente. O fotodiodo e o processo de produção do par "elétron-buraco", através de um fóton incidente, estão esquematizados na figura 3.3.

Assim, o fotodiodo, operando reversamente polarizado, difere do diodo 


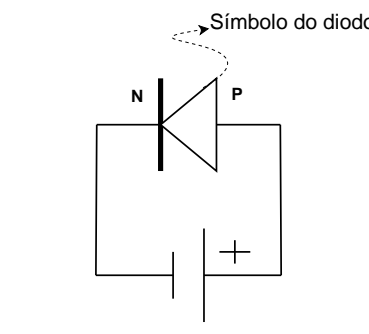

Polarizado diretamente

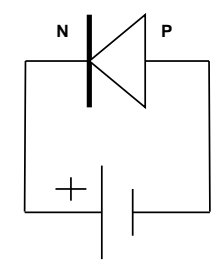

Polarizado reversamente

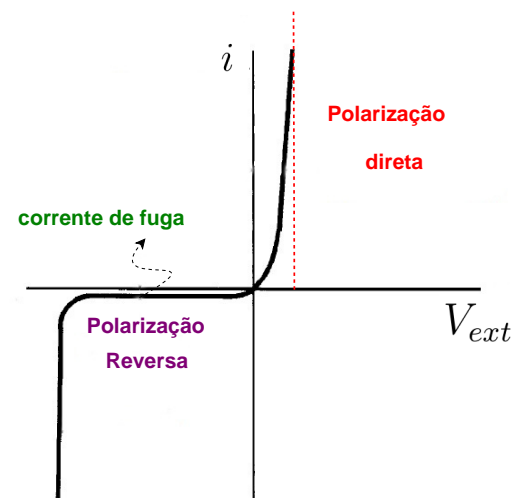

(b)

(a)

Figura 3.2: Na parte (a), é visto as duas formas de ligação de um diodo. Quando diretamente polarizado, há a passagem livre de corrente elétrica através dele. Ao contrário, no caso reversamente polarizado, não há passagem de corrente considerável. Na parte (b), é ilustrada a curva característica do diodo que descreve graficamente esse comportamento em relação à passagem de corrente.

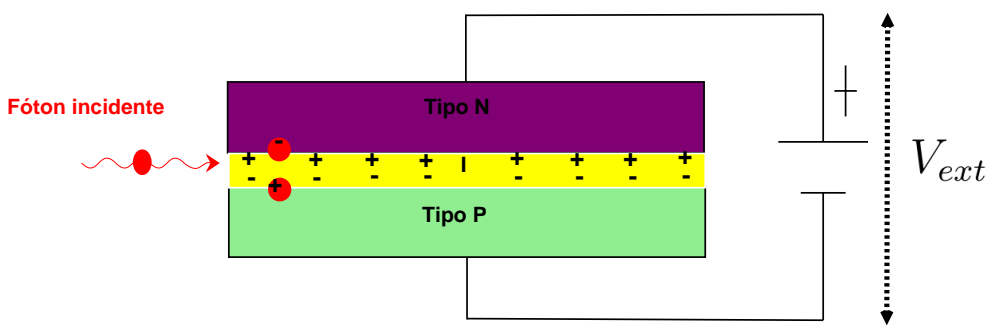

Figura 3.3: Representação do fotodiodo tipo PIN reversamente polarizado. Cada fóton da luz incidente no fotodiodo produz um par "elétron-buraco" que é acelerado através da tensão externa aplicada. 
comum apenas pela presença de uma corrente internamente gerada devido a sua exposição à luz [21]. A fotocorrente gerada nesse processo varia no tempo e é proporcional ao número de elétrons gerados, ou seja, é proporcional ao número de fótons incidentes e carrega em si a informação quântica da luz. Dessa forma, o fotodiodo é um dispositivo eletrônico que mede essencialmente a intensidade de um campo.

Da mesma forma como o diodo, o fotodiodo também apresenta uma curva característica, corrente versus tensão, com a diferença que existe uma curva para cada valor da intensidade da luz incidente. A Figura 3.4 mostra esquematicamente uma família dessas curvas.

À medida que o valor da intensidade da luz aumenta, o fotodiodo produz uma fotocorrente maior em módulo. Entretanto, quando não há incidência de luz no fotodiodo e, mesmo assim, ele ainda está reversamente polarizado, aparece, exatamente como no diodo convencional, uma corrente de fuga. Essa corrente de fuga gera também um ruído a mais na fotocorrente, conhecido na literatura [18] como "dark noise", que, juntamente com outros ${ }^{2}$ ruídos que não se originam da natureza quântica da luz, é descontado das medidas. Assim, a corrente de fuga é a responsável pelo ruído eletrônico do fotodiodo que deve ser necessariamente desprezível para não mascarar o ruído quântico.

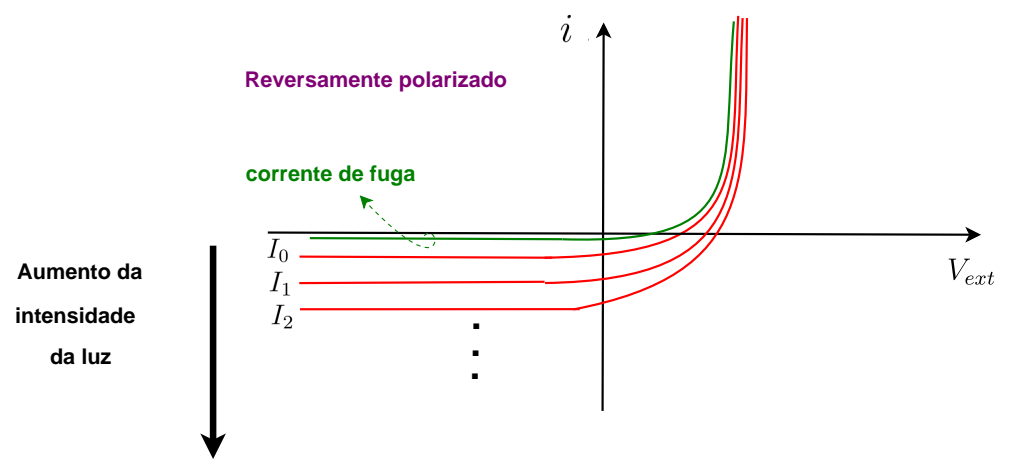

Figura 3.4: Curva caracterítica de um fotodiodo reversamente polarizado. Cada curva resposta, em vermelho, corresponde a uma certa intensidade de luz incidente que gera uma determinada fotocorrente. À medida que a intensidade do campo aumenta, $I_{0}<I_{1}<I_{2} \ldots$, o módulo da fotocorrente também aumenta. A curva verde representa a situação em que o fotodiodo está submetido à uma tensão elétrica, porém sem a incidência de luz.

Em termos da quantização do campo, o fotodiodo mede diretamente o operador número $\hat{a}^{\dagger} \hat{a}$, em que o operador $\hat{a}$ é o operador de aniquilação de

\footnotetext{
${ }^{2}$ Veremos, mais adiante, outro tipo de ruído que intefere nas nossas medidas, conhecido como ruído Johnson ou térmico.
} 
um campo monomodo incidente, por exemplo. Dessa forma, o valor médio da fotocorrente medida, $\bar{i}(t)$, sem amplificação eletrônica, é igual a [5]:

$$
\bar{i}(t)=e\left\langle\hat{a}^{\dagger} \hat{a}\right\rangle,
$$

$e$ a carga elétrica do elétron. Esse valor médio, $\bar{i}(t)$, é a parte $D C$ do sinal da fotocorrente.

Embora o material fotosensível $I$ seja eficiente na medida da intensidade do campo elétrico incidente, ele não permite a medida direta da fase do campo. A razão disso é que não existe fotodiodos que respondam a frequências tão altas quanto as frequências óticas [20], da ordem de $10^{15} \mathrm{~Hz}$, isso torna a medida direta da fase do campo impossível. Para acessá-la experimentalmente é necessário o uso de técnicas interferométricas tais como a detecção homodina e auto-homodina, conforme veremos descritas nas próximas seções.

Além da parte $D C$, a fotocorrente gerada apresenta uma parte $H F$, ou de altas frequências, onde toda a informação sobre o estado quântico da luz detectada encontra-se. É fundamental o tratamento eletrônico do sinal gerado pela fotocorrente que irá separá-lo em dois sinais conforme a frequência, $A C$ e $H F$, e amplificá-lo. A descrição de tais procedimentos eletrônicos será feita mais adiante ao longo do texto.

\subsubsection{Eficiência quântica}

Um parâmetro importante a se considerar ao decidir-se pelo uso de um dado fotodiodo em um experimento é a sua eficiência quântica $\eta[5,22]$. A eficiência diz respeito à razão de quantos fótons da luz detectada são convertidos em elétrons livres pelo material semicondutor do fotodiodo.

Ao incidir no fotodetector, os fótons da luz são absorvidos pelo fotodiodo que produz elétrons livres. Esses elétrons formam uma fotocorrente proporcional à potência $P(t)$ da luz e que carrega a informação quântica presente na luz. Para uma eficiência igual a 1 , podemos afirmar que a estatítica desses elétrons produzidos é exatamente a mesma estatística dos fótons da luz incidente, o que significa que as flutuações da fotocorrente estão diretamente conectadas às flutuações da própria luz e, assim, podem ser utilizadas para investigar os aspectos quânticos dela [18].

Sabemos que essa fotocorrente, $i(t)$, é a razão entre o número de elétrons livres produzidos, $n_{e}$, e um dado intervalo de tempo $\Delta t$ :

$$
i(t)=\frac{n_{e} e}{\Delta t},
$$

em que e é a carga elétrica de um único elétron igual a $1,6 X 10^{-19} \mathrm{C}$. 
Além disso, a potência da luz incidente no fotodetector, $P(t)$, é a razão entre a energia dos fótons da luz, $n_{f} h \nu$, e o mesmo intervalo de tempo, igual a:

$$
P(t)=\frac{n_{f} h \nu}{\Delta t}=\frac{n_{f} h c}{\lambda \Delta t}
$$

em que $n_{f}$ é o número de elétrons livres que formam a fotocorrente, $\nu$ é a frequência da luz, $h$ a contante de Planck $\left(6,63 X 10^{-34} \mathrm{~J} . \mathrm{s}\right), c$ a velocidade da luz $\left(3 X 10^{8} \mathrm{~m} / \mathrm{s}\right)$ e $\lambda$ é o comprimento de onda da luz.

A eficiência quântica é definida como a razão entre o número de elétrons produzidos com a exposição do detector à luz e o número de fótons:

$$
\eta=\frac{n_{e}}{n_{f}}
$$

Assim, das equações (3.2), (3.3) e (3.4), temos:

$$
\eta=\left(\frac{h c}{\lambda e}\right) \frac{i(t)}{P(t)}
$$

Como os feixes que detectamos no experimento estão na faixa de $1064 \mathrm{~nm}$, a equação (3.5), finalmente, é simplificada por:

$$
\frac{i(t)}{P(t)}=(0,86) \eta=R
$$

medidos, no sistema internacional, em Ampere por Watt, $\frac{A}{W}$. Assim, a relação entre as grandezas observadas $i(t)$ e $P(t)$ é linear e igual a uma constante de proporcionalidade, $R$, denominada responsividade do fotodiodo.

Em outras palavras, a responsividade refere-se à sensibilidade de resposta elétrica do fotodiodo à incidência de luz e depende de certas características do material semicondutor do fotodiodo.

$\mathrm{Na}$ nossa montagem atual, trabalhamos principalmente com feixes na região do infravermelho e empregamos fotodiodos da marca Epitaxx, do tipo ETX300. Para ilustrar, é vista na Figura 3.5 a curva da responsividade desse tipo de fotodiodo. De acordo com essa curva fornecida pelo fabricante e a equação (3.6), concluimos que a eficiência quântica do fotodiodo é de $90 \%$. Somente com a intenção de confirmar experimentalmente tal valor, realizamos as medidas ${ }^{3} P(t)$ versus $i(t)$ e obtemos exatamente o mesmo valor. Essas medidas são vistas no capítulo 6 referente à nossa montagem.

\footnotetext{
${ }^{3} \mathrm{Na}$ verdade, como explicado mais adiante, medimos $P(t)$ versus $D C$, sinal de tensão. Isso porque o circuito do detector transforma corrente em um sinal de tensão.
} 


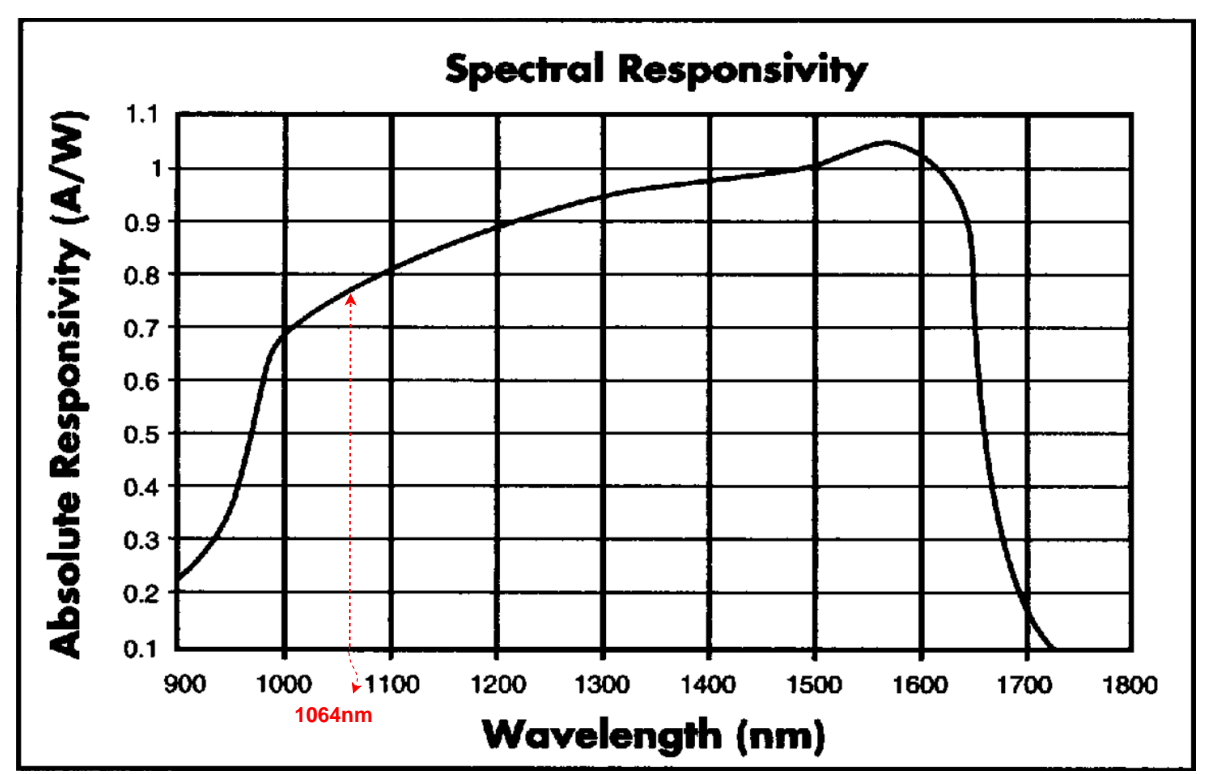

Figura 3.5: Curva de responsividade fornecida pelo fabricante [19] do fotodiodo ETX300 encontrado no nosso experimento. Em destaque, a responsividade, em torno de $0.78 \mathrm{~A} / \mathrm{W}$, referente ao comprimento de onda $1064 \mathrm{~nm}$ utilizado.

\subsubsection{Circuito simplificado do detector}

A fotocorrente gerada no processo de detecção da luz pelo fotodiodo precisa, como dito anteriormente, passar por um tratamento eletrônico para que, finalmente, seja capaz de fornecer a informação contida no estado quântico da luz.

Esse tratatamento consiste basicamente de filtragens e amplificações eletrônicas (ver Apêndice A). Para isso, faz-se necessário a utilização de outros componentes eletrônicos tais como resistores, capacitores e amplificadores operacionais associados ao fotodiodo. Assim, o detector de luz compreende o fotodiodo e todo o circuito eletrônico envolvido no tratamento do seu sinal.

O desenho simplificado do circuito do detector [22] que usamos no laboratório é vizualizado na Figura 3.6. O fotodiodo ETX300, operando de forma reversa, absorve a luz incidente e gera uma fotocorrente $i_{\text {foto }}$. Essa fotocorrente é o resultado da interferência de inúmeras fotocorrentes em diversas frequências, baixas e altas, que define uma certa largura de banda $B W$, dependendo do fotodiodo. Da mesma forma como visto no contexto de cavidades óticas, o parâmetro $B W$ aqui serve para indicar o intervalo de frequências desse sinal $i_{\text {foto }}$.

Como visto no circuito da Figura 3.6, após a geração de $i_{\text {foto }}$, existe um 
filtro RC passa alta que separa os sinais através da sua frequência de corte. De acordo com a equação (A.9), a função de ganho desse filtro passa alta é mostrada na Figura 3.7.

Após essa primeira filtragem, a corrente $i_{\text {foto }}$ se divide em $i_{H F}$ e $i_{D C}$. Cada uma dessas correntes, antes de servirem no processo de medida, passam ainda por uma etapa de amplificação e mais uma etapa de filtragem que acaba por transformá-la em um sinal de tensão (ver Apêndice B).

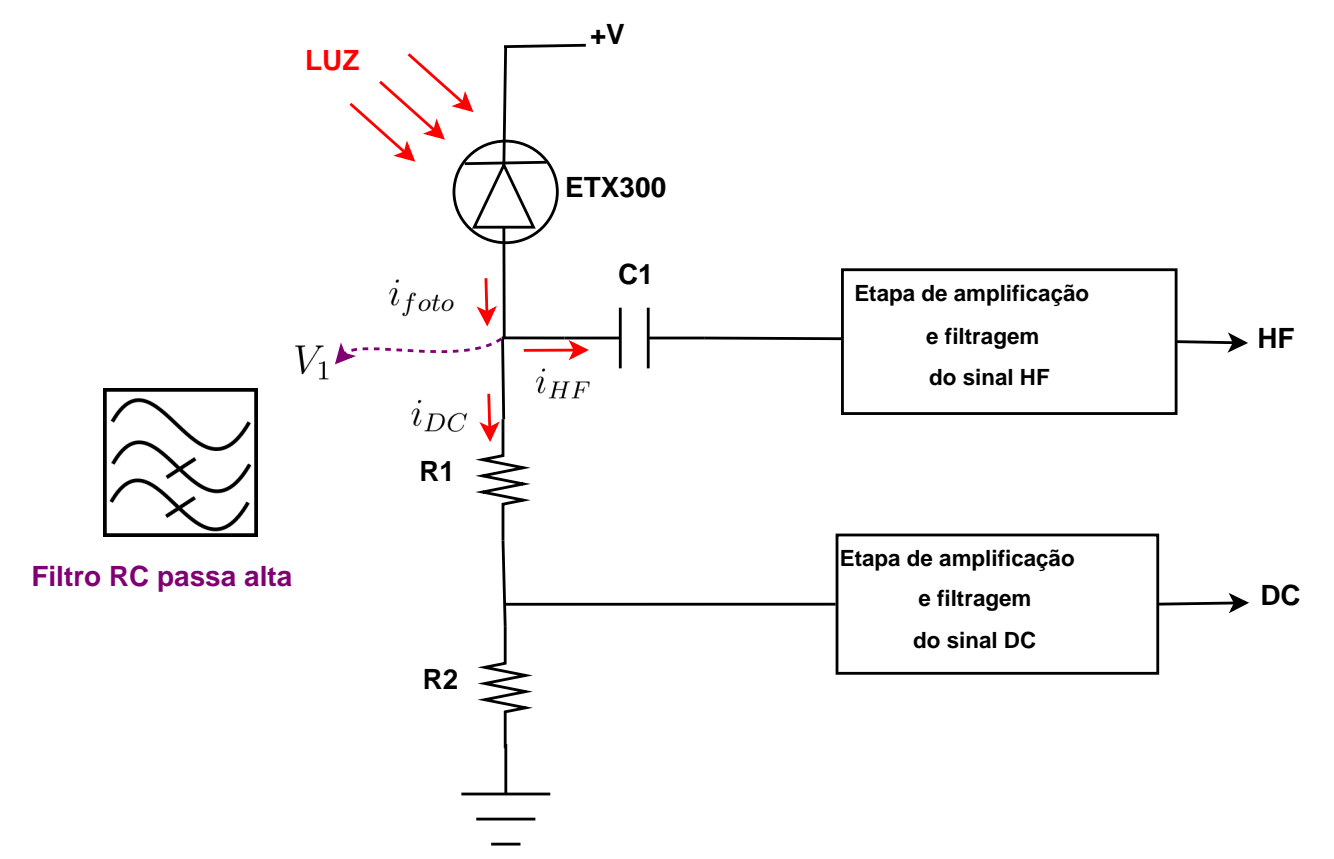

Figura 3.6: Circuito simplificado do detector. A luz incide no fotodiodo ETX300, reversamente polarizado, que produz uma fotocorrente $i_{\text {foto }}$ que contém sinais de altas e baixas frequências. Essa fotocorrente passa pelo filtro passa alta $R C$ de frequência de corte, $f_{\text {corte, }}$, igual a $15 \mathrm{MHz}$ e a corrente se divide em: $i_{H F}$ e $i_{D C}$. Cada uma dessas novas correntes são amplificadas e transformadas em tensões, antes de ser usadas nas medidas. Valores atuais dos componentes nos nossos detectores: $C_{1}=27 p F, R_{1}=385 \Omega$ e $R_{2}=100 \Omega$.

\subsection{Detecção homodina balanceada}

Recapitulando o que discutimos anteriormente, os fotodetectores são dispositivos eletrônicos sensíveis à medida da intensidade da luz. Entretanto, não são rápidos o bastante para responder à fase ótica do campo eletromagnético. 


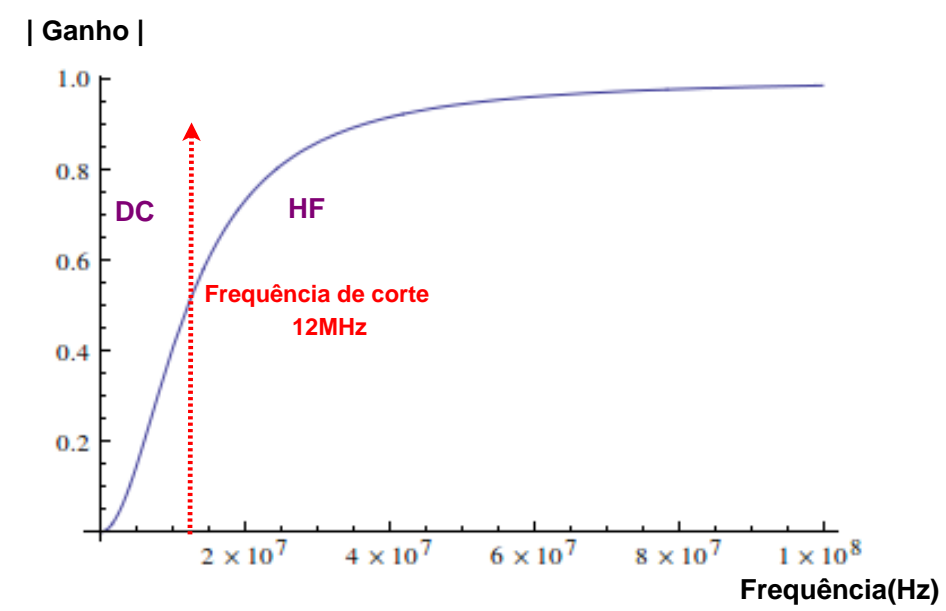

Figura 3.7: Curva de ganho do filtro passa-alta $R C$ empregado no detector para separar os sinais de alta e baixa frequência. Frequência de corte igual a $12 \mathrm{MHz}$. Valores atuais dos componentes nos nossos detectores: $C_{1}=$ $27 p F, R_{1}=385 \Omega$ e $R_{2}=100 \Omega$.

Sendo assim, faz-se urgente o uso de algumas técnicas para acessar não só a medida da intensidade do campo, mas também da sua fase.

O propósito desta seção é justamente fornecer uma visão matemática simplificada do método de medida das quadraturas de um campo mais usado e conhecido na literatura $[5,10,11,12,18,21,23]$, denominado detecção homodina balanceada.

A informação quântica contida em um campo, a princípio, pode ser acessada experimentalmente através da análise do espectro de ruído da fotocorrente gerada por um detector quando exposto ao mesmo.

A medida direta dessa fotocorrente e seu respectivo espectro de ruído fornece somente a medida da quadratura $\hat{X}_{\phi}$ do campo, associada à intensidade, e do seu ruído. Entretanto, para medir $\hat{Y}_{\phi}$, associada à fase do campo, é necessário montar um esquema interferométrico, onde o controle de fase $\phi$ é feito através da variação do comprimento de um dos braços do interferômetro. A depender do valor da fase, tem-se a medida de uma das quadraturas do estado da luz.

A ideia central da detecção homodina é interferir em um BS, do tipo 50:50, o campo, que se deseja medir, com outro campo coerente de aproximadamente mesma frequência e muito mais intenso, conhecido como oscilador local (LO). O campo resultante dessa interferência entre o oscilador e o campo a ser medido é caracterizado por modulações de fase e amplitude. A detecção dessas modulações óticas geram fotocorrentes cuja soma/subtração 
está relacionada diretamente aos observáveis $\hat{X}_{\phi}$ e $\hat{Y}_{\phi}$.

Inicialmente vamos considerar que o campo a ser medido está em um único modo de frequência e em uma dada polarização específica. Além disso, supõe-se que o estado quântico do campo é puro e não muda durante toda a realização da medida.

A suposição de que o estado é puro não é rigorosamente verdadeira, pois os estados quânticos da luz são misturas estatísticas, descritas corretamente através dos seus operadores densidades $\hat{\rho}$. A associação de estados quânticos ou misturas estatísticas a distribuições de probabilidade é feita por meio da reconstrução da função de Wigner que os representa. Nas nossas medidas, não faremos a reconstrução dessa função, pois consideraremos que tal função é do tipo gaussiana e, na realidade, o que temos é um corte topográfico dela.

Fisicamente, veremos também que tal campo monomodo, ou monocromático, não existe. Porém, assim como vimos no capítulo 2 sobre a onda plana, simplificações como essa ajudam na compreensão da essência dos fenômenos físicos.

Todo feixe é, na realidade, uma soma de infinitos modos de frequência e sua energia está distruibuída por todos esses modos. Esse conceito ficará mais claro nas próximas seções onde passaremos a abordar campos multimodos.

Somente para facilitar, será usada em toda essa seção a representação de Schrödinger, em que a dinâmica quântica ocorre com a evolução temporal dos estados quânticos e não dos operadores observáveis. Na próxima seção, entretanto, consideraremos campos multimodos e passaremos a adotar a representação de Heisenberg.

Como o que se mede são somente as flutuações das fotocorrentes geradas no processo de detecção dos campos, essas podem ser interpretadas tanto como resultantes da dinâmica temporal dos estados quânticos quanto da dinâmica dos observáveis $\hat{X}_{\phi}\left(\right.$ ou $\left.\hat{Y}_{\phi}\right)$. Dessa forma, não existe uma representação privilegiada e sim mais conveniente.

Ao longo de todo esse texto, as notações ${ }^{4}$ adotadas para a média e variância serão as seguintes respectivamente: $\langle($ Operador $)\rangle,\left\langle\Delta^{2}(\right.$ Operador $\left.)\right\rangle$ para operadores quânticos, e $\overline{v . a}, \operatorname{Var}(v . a)$ para variáveis aleatórias (v.a) estatísticas, no caso, as próprias medidas reais obtidas no laboratório.

A Figura 3.8 ilustra o esquema da detecção homodina balanceada em que os campos de entrada são representados genericamente por $\hat{a}$ e $\hat{b}$ e os campos de saída por $\hat{c}$ e $\hat{d}$. O campo $\hat{a}$, cujo estado é denotado por $\left|\psi_{a}\right\rangle$, é o que se deseja medir as quadraturas, e o campo $\hat{b}$ é o oscilador local, cujo estado é

\footnotetext{
${ }^{4} \mathrm{O}$ uso de notações distintas para objetos matemáticos aparentemente semelhantes é uma questão de preferência pessoal para ressaltar qual o espaço a que se aplica: Hilbert, no caso quântico, ou espaço amostral, no caso estatístico.
} 
coerente e denotado por $|\beta\rangle$, de amplitude $\beta^{2}, \beta$ dado por um número real para facilitar.

Para detectar os campos de saída $\hat{c}$ e $\hat{d}$, são posicionados os detectores $\operatorname{det}_{c}$ e $\operatorname{det}_{d} \operatorname{logo}$ após o BS. Supõe-se que tais detectores possuam eficiência ${ }^{5}$ quântica $\eta$ igual a 1 . Essa suposição permite afirmar que a estatística dos fótons do feixe incidente no fotodetector é idêntica à estatística dos elétrons que formam a fotocorrente e, assim, as flutuações desta está diretamente ligada às propriedades quânticas da luz que queremos estudar [18].

Como será demonstrado a seguir, as medidas das quadraturas $\hat{X}_{\phi}$ e $\hat{Y}_{\phi}$ do campo eletromagnético e dos seus ruídos são feitas através da análise da subtração das fotocorrentes $i_{c}$ e $i_{d}$ medidas pelos detectores $\operatorname{det}_{c}$ e $\operatorname{det}_{d}$ respectivamente. No esquema da figura, é visto o dispositivo eletrônico que realizada a soma/subtração dessas fotocorrentes, denominado por somador/subtrator.

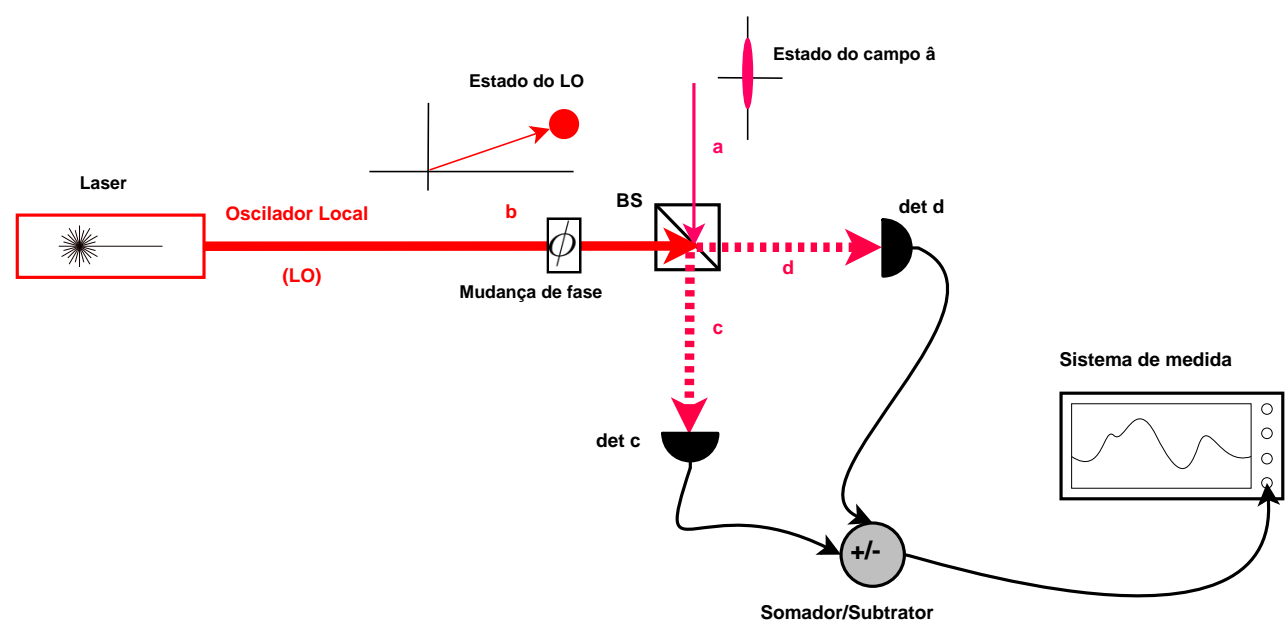

Figura 3.8: Esquema ilustrativo do método de detecção homodina balanceada. Dois campos $\hat{a}$ e $\hat{b}$, muito mais intenso que $\hat{a}$, incidem em um BS 50:50, resultando nos campos $\hat{c}$ e $\hat{d}$ que são detectados respectivamente pelos detectores $\operatorname{det}_{c}$ e $\operatorname{det}_{d}$. No dispositivo eletrônico, denotado por $+/-$, essas fotocorrentes são somadas ou subtraídas e enviada para o sistema de medida.

As relações entre os modos do campos de entrada $(\hat{a}$ e $\hat{b})$ e os modos de saída $(\hat{c}$ e $\hat{d})$ no BS são fornecidas ${ }^{6}$ por:

$$
\hat{c}=\frac{1}{\sqrt{2}}(\hat{a}+\hat{b}) \quad e \quad \hat{d}=\frac{1}{\sqrt{2}}(\hat{b}-\hat{a})
$$

\footnotetext{
${ }^{5}$ Veremos que os nossos detectores, na prática, são $90 \%$ eficientes, o que acrescenta uma possível fonte de erro em nossas medidas.

${ }^{6}$ Relações de BS conhecidas, dada a quantização do campo.
} 
Os campos de saída detectados por $\operatorname{det}_{c}$ e $\operatorname{det}_{d}$ fornecem como resultado as fotocorrentes $i_{c}$ e $i_{d}$, cujas médias $\overline{i_{c}}$ e $\overline{i_{d}}$ são proporcionais às médias do operadores números $\left\langle\hat{n}_{c}\right\rangle$ e $\left\langle\hat{n}_{d}\right\rangle$ a menos de uma contante $k_{\text {eletro }}$, relacionada ao ganho eletrônico. Essa constante depende do ganho do sistema eletrônico envolvido na medida e será omitida, para simplificar, durante toda a demonstração que se segue e retomada somente depois na calibração do sistema de medida.

De posse das relações (3.7), é possível escrever $\overline{i_{c}}$ e $\overline{i_{d}}$ em termos dos modos dos campos de entrada $\hat{a}$ e $\hat{b}$ :

$$
\begin{aligned}
& \overline{i_{c}}=\left\langle\hat{n}_{c}\right\rangle=\left\langle\hat{c}^{\dagger} \hat{c}\right\rangle=\left\langle\frac{1}{2}\left(\hat{a}^{\dagger} \hat{a}+\hat{a}^{\dagger} \hat{b}+\hat{b}^{\dagger} \hat{a}+\hat{b}^{\dagger} \hat{b}\right)\right\rangle \\
& \overline{i_{d}}=\left\langle\hat{n}_{d}\right\rangle=\left\langle\hat{d}^{\dagger} \hat{d}\right\rangle=\left\langle\frac{1}{2}\left(\hat{a}^{\dagger} \hat{a}-\hat{a}^{\dagger} \hat{b}-\hat{b}^{\dagger} \hat{a}+\hat{b}^{\dagger} \hat{b}\right)\right\rangle
\end{aligned}
$$

Subtraindo (3.8) de (3.9), calcula-se a fotocorrente obtida pelo subtrator $i_{c}-i_{d}$ escrita em termos dos modos $\hat{a}$ e $\hat{b}$ :

$$
\overline{i_{c}-i_{d}}=\left\langle\hat{c}^{\dagger} \hat{c}-\hat{d}^{\dagger} \hat{d}\right\rangle=\left\langle\hat{a}^{\dagger} \hat{b}+\hat{b}^{\dagger} \hat{a}\right\rangle
$$

O oscilador local $\hat{b}$, como já mencionado, é um campo, cujo estado é coerente, de mesma frequência $\omega$ do campo $\hat{a}$, porém muito mais intenso. É conveniente representar o estado coerente por $|\beta\rangle$ de forma que sob ação do operador $\hat{b}^{7}$ é igual a:

$$
\hat{b}|\beta\rangle=\beta|\beta\rangle
$$

O estado total do sistema, denotado por $|\psi\rangle$, formado pelos estados dos campos de entrada, é igual ao produto tensorial entre o estado do campo $\hat{a}$, $\left|\psi_{a}\right\rangle$, e o estado coerente do campo $\hat{b},|\beta\rangle$, e não muda depois da passagem dos campos pelo BS:

$$
|\psi\rangle=|\beta\rangle \otimes\left|\psi_{a}\right\rangle
$$

A fase de $\hat{b}$ em relação ao campo $\hat{a}$ é denotada por $\phi$ tal que ele pode ser substituído por $\hat{b} e^{i \phi}$ em (3.10):

$$
\overline{i_{c}-i_{d}}=\left\langle\hat{a}^{\dagger} \hat{b} e^{i \phi}+\hat{b}^{\dagger} e^{-i \phi} \hat{a}\right\rangle
$$

Substituindo (3.11) e os operadores $\hat{a}$ e $\hat{b}$ em (3.13), obtém-se:

$$
\begin{aligned}
& \overline{i_{c}-i_{d}}=\left\langle\psi\left|\hat{a}^{\dagger} \hat{b} e^{i \phi}\right| \psi\right\rangle+\left\langle\psi\left|\hat{b}^{\dagger} e^{-i \phi} \hat{a}\right| \psi\right\rangle \\
= & \beta\left(\left\langle\psi\left|\hat{a}^{\dagger} e^{i \phi}+\hat{a} e^{-i \phi}\right| \psi\right\rangle\right)
\end{aligned}
$$

\footnotetext{
${ }^{7}$ Lembrando-se que o vetor $|\beta\rangle$ é autoestado do operador aniquilação $\hat{b}$.
} 
Sabendo-se que o operador quadratura $\hat{X}_{\phi}$, na representação de Schrödinger, é igual a:

$$
\hat{X}_{\phi}=\hat{a}^{\dagger} e^{i \phi}+\hat{a} e^{-i \phi}
$$

é possível reescrever (3.14) como:

$$
\overline{i_{c}-i_{d}}=\beta\left\langle\psi\left|\hat{X}_{\phi}\right| \psi\right\rangle
$$

Como visto em (3.16), a medida indireta da média da quadratura $\hat{X}_{\phi}$ está relacionada à subtração das fotocorrentes $i_{c}$ e $i_{d}$ a menos de um fator $\frac{1}{\beta}$. A medida da quadratura conjugada $\hat{Y}_{\phi}$ é feita variando-se a fase $\phi$ do oscilador local (ou campo $\hat{b}$ ) em relação ao campo $\hat{a}$. Assim, quando o valor de $\phi$ for igual a 0 , tem-se a quadratura $\hat{X}$, quando $\phi$ for igual a $\frac{\pi}{2}$, tem-se a quadratura $\hat{Y}$.

De forma análoga ao cálculo anterior, é possível calcular a variância (Var) da subtração das fotocorrentes $\operatorname{Var}\left(i_{c}-i_{d}\right)$, sabendo-se que:

$$
\operatorname{Var}\left(i_{c}-i_{d}\right)=\overline{\left(i_{c}-i_{d}\right)^{2}}-\left(\overline{i_{c}-i_{d}}\right)^{2}
$$

Com (3.13), calcula-se o primeiro termo da (3.17):

$$
\begin{gathered}
\overline{\left(i_{c}-i_{d}\right)^{2}}=\left\langle\psi\left|\left(\hat{a}^{\dagger} \hat{b} e^{i \phi}+\hat{b}^{\dagger} e^{-i \phi} \hat{a}\right)^{2}\right| \psi\right\rangle= \\
=\left\langle\psi\left|\left(\hat{a}^{\dagger}\right)^{2} \hat{b}^{2} e^{2 i \phi}+\hat{a}^{\dagger} \hat{b} \hat{b}^{\dagger} \hat{a}+\hat{b}^{\dagger} \hat{a} \hat{a}^{\dagger} \hat{b}+\left(\hat{b}^{\dagger}\right)^{2} \hat{a}^{2} e^{-2 i \phi}\right| \psi\right\rangle
\end{gathered}
$$

Para facilitar o cálculo de (3.18), faz-se necessário o uso do comutador $\left[\hat{a}^{\dagger} \hat{b}, \hat{b}^{\dagger} \hat{a}\right]$ que é igual $\mathrm{a}^{8}$.

$$
\begin{aligned}
& {\left[\hat{a}^{\dagger} \hat{b}, \hat{b}^{\dagger} \hat{a}\right]=\hat{a}^{\dagger}\left[\hat{b}, \hat{b}^{\dagger} \hat{a}\right]+\left[\hat{a}^{\dagger}, \hat{b}^{\dagger} \hat{a}\right] \hat{b}=} \\
= & \hat{a}^{\dagger}\left(\hat{b}^{\dagger}[\hat{b}, \hat{a}]+\left[\hat{b}, \hat{b}^{\dagger}\right] \hat{a}\right)+\left(\hat{b}^{\dagger}\left[\hat{a}^{\dagger}, \hat{a}\right]+\left[\hat{a}^{\dagger}, \hat{b}^{\dagger}\right] \hat{a}\right) \hat{b}
\end{aligned}
$$

Com as relações de comutação entre os operadores criação e aniquilação dados por (2.3), simplifica-se a equação (3.19):

$$
\left[\hat{a}^{\dagger} \hat{b}, \hat{b}^{\dagger} \hat{a}\right]=\hat{a}^{\dagger} \hat{a}-\hat{b}^{\dagger} \hat{b}
$$

Assim, o operador $\hat{a}^{\dagger} \hat{b}^{\dagger} \hat{a}$ é obtido da (3.20):

$$
\hat{a}^{\dagger} \hat{b} \hat{b}^{\dagger} \hat{a}=\hat{a}^{\dagger} \hat{a}-\hat{b}^{\dagger} \hat{b}+\hat{b}^{\dagger} \hat{a} \hat{a}^{\dagger} \hat{b}
$$

${ }^{8}$ Usou-se a seguinte propriedade dos comutadores : Sejam $\hat{a}, \hat{b}$ e $\hat{c}$ operadores quaisquer, então $[\hat{a}, \hat{b} \hat{c}]=\hat{b}[\hat{a}, \hat{c}]+[\hat{a}, \hat{b}] \hat{c}$ 
Através da equação (3.21), encontra-se o valor do seguinte operador em (3.18):

$$
\hat{a}^{\dagger} \hat{b} \hat{b}^{\dagger} \hat{a}+\hat{b}^{\dagger} \hat{a} \hat{a}^{\dagger} \hat{b}=\hat{a}^{\dagger} \hat{a}-\hat{b}^{\dagger} \hat{b}+2 \hat{b}^{\dagger} \hat{a} \hat{a}^{\dagger} \hat{b}
$$

Substituindo-se as equações (3.22) e $\hat{a} \hat{a}^{\dagger}=1+\hat{a}^{\dagger} \hat{a}$ em (3.18) e lembrando-se da hipótese inicial de que $|\beta\rangle$ é um estado coerente e vale a equação de autovalor $(3.11)$, obtém-se o valor médio de $\left(i_{c}-i_{d}\right)^{2}$ :

$$
\begin{aligned}
\overline{\left(i_{c}-i_{d}\right)^{2}} & =\beta^{2}\left\langle\psi\left|\left(\hat{a}^{\dagger}\right)^{2} e^{2 i \phi}\right| \psi\right\rangle+\left\langle\psi\left|\hat{a}^{\dagger} \hat{a}\right| \psi\right\rangle-\beta^{2}+ \\
& +\beta^{2}\left\langle\psi\left|\left(2+2 \hat{a}^{\dagger} \hat{a}\right)\right| \psi\right\rangle+\beta^{2}\left\langle\psi\left|\hat{a}^{2} e^{-2 i \phi}\right| \psi\right\rangle
\end{aligned}
$$

Tendo em vista que:

$$
\hat{X}_{\phi}^{2}=\left(\hat{a}^{\dagger}\right)^{2} e^{2 i \phi}+2 \hat{a}^{\dagger} \hat{a}+1+\hat{a}^{2} e^{-2 i \phi},
$$

reescreve-se a (3.23) em termos do operador $\hat{X}_{\phi}^{2}$, resultando na equação:

$$
\overline{\left(i_{c}-i_{d}\right)^{2}}=\beta^{2}\left\langle\psi_{a}\left|\hat{X}_{\phi}^{2}\right| \psi_{a}\right\rangle+\left\langle\psi_{a}\left|\hat{a}^{\dagger} \hat{a}\right| \psi_{a}\right\rangle
$$

A variância $\operatorname{Var}\left(i_{c}-i_{d}\right)$ é, finalmente, obtida substituindo as equações (3.16) e (3.25) na (3.17):

$$
\operatorname{Var}\left(i_{c}-i_{d}\right)=\beta^{2}\left\langle\psi_{a}\left|\hat{X}_{\phi}^{2}\right| \psi_{a}\right\rangle+\left\langle\psi_{a}\left|\hat{a}^{\dagger} \hat{a}\right| \psi_{a}\right\rangle-\beta^{2}\left\langle\psi_{a}\left|\hat{X}_{\phi}\right| \psi_{a}\right\rangle^{2}
$$

Como, por hipótese, a intensidade do campo â é muito menor que a intensidade do oscilador local $\hat{b}$, então $\left\langle\psi\left|\hat{a}^{\dagger} \hat{a}\right| \psi\right\rangle$ é desprezível e a variância $\operatorname{Var}\left(i_{c}-i_{d}\right)$ é igual a:

$$
\operatorname{Var}\left(i_{c}-i_{d}\right)=\beta^{2}\left\langle\psi_{a}\left|\Delta^{2} \hat{X}_{\phi}\right| \psi_{a}\right\rangle
$$

Assim, as variâncias das quadraturas $\hat{X}_{\phi}$ e $\hat{Y}_{\phi}$, ou espectro de ruído, são obtidas separadamente através da medida da variância da subtração das fotocorrentes, dada a fase $\phi$ do oscilador local. Da mesma forma como nas medidas das médias, se $\phi=0$, tem-se a variância do observável $\hat{X}_{\phi}$, por outro lado, se $\phi=\frac{\pi}{2}$, obtém-se a variância do observável $\hat{Y}_{\phi}$.

Ainda de acordo com a equação (3.27), quanto mais intenso for o oscilador local $\left(\beta^{2}\right)$, maior será a variância da subtração das fotocorrentes medida. Entretanto, as flutuações presentes nela são originadas, exclusivamente, do campo â e não do oscilador local [18].

Ao longo de toda essa discussão, percebeu-se o papel fundamental exercido pela fase $\phi$ do oscilador local. É através de sua variação ${ }^{9}$, controlada

\footnotetext{
${ }^{9} \mathrm{O}$ controle da fase do oscilador local também pode ser feito através da rotação de uma lâmina de vidro [18].
} 
por um PZT (atuador piezoelétrico) acoplado a um espelho, que podemos medir as quadraturas do campo.

Para servir tão bem como selecionador de fase, o oscilador local deve necessariamente ter sua fase travada em relação à fase do campo a ser medido $\hat{a}$ [18]. Na prática, em uma deteç̧ão do tipo homodina, o oscilador e o campo $\hat{a}$ tem aproximadamente a mesma frequência ótica e são criados pela mesma fonte de luz, o que garante uma relação de fase bem estabelecida entre eles.

\subsection{Rotação da elipse de ruído}

De acordo com o que foi descrito previamente, o acesso experimental das quadraturas de um campo eletromagnético ocorre somente através da medida da sua intensidade, $\hat{X}_{\phi}$. Para termos acesso à quadratura $\hat{Y}_{\phi}$ é necessário variar a fase $\phi$ do oscilador local convenientemente de $\phi=\frac{\pi}{2}$. Uma forma de encarar isso, considerando a representação do estado no espaço de fase, é dizer que rotacionamos os eixos $\hat{X}_{\phi}$ e $\hat{Y}_{\phi}$ a fim de projetar o ruído de fase totalmente em ruído de intensidade para tornar a medida de fase do campo possível.

Esse tipo de transformação do espaço de fase é dito passivo [9] porque foi aplicado ao referencial $\hat{X}_{\phi}-\hat{Y}_{\phi}$ no qual o estado do campo $\hat{a}$ é descrito. Entretanto, essa transformação espacial também pode ser encarada alternativamente como aplicada ao próprio sistema físico, em que é a própria elipse de ruído que rotaciona. Tal enfoque é dito ativo . Independente de qual enfoque adotado, a dinâmica do campo é invariante sob tais transformações e a diferença de um enfoque para outro implica somente em uma diferença de fase [9]. Ao longo de toda essa discussão sempre será adotado o ponto de vista passivo.

A Figura 3.9 mostra um exemplo de rotação de um possível estado $\left|\psi_{a}\right\rangle$ do campo $\hat{a}$, vácuo comprimido. Nesse caso específico, a compressão de ruído encontra-se em $\hat{X}_{\phi=0}$ e o excesso de ruído em $\hat{Y}_{\phi=0}$. Ao variar a fase do oscilador local, os eixos $\hat{X}_{\phi=0}-\hat{Y}_{\phi=0}$ são girados e, por consequência, a elipse aparece rotacionada no novo espaço de fase. A elipse de ruído nessa nova configuração do espaço de fase permite que o ruído de fase seja, finalmente, medido.

Por abuso de linguagem, falaremos sempre em rotação da elipse indicando, na verdade, a rotação dos eixos que a descrevem no espaço de fase.

Até agora falamos da rotação da elipse de ruído tomando como referência o espaço de fase do campo $\hat{a}$, inicialmente com $\phi=0$, para facilitar a abordagem. Porém, sabemos que ocorre uma interferência entre os campos $\hat{a}$ e o oscilador local no BS, sendo a detecção desse campo resultante que fornece 


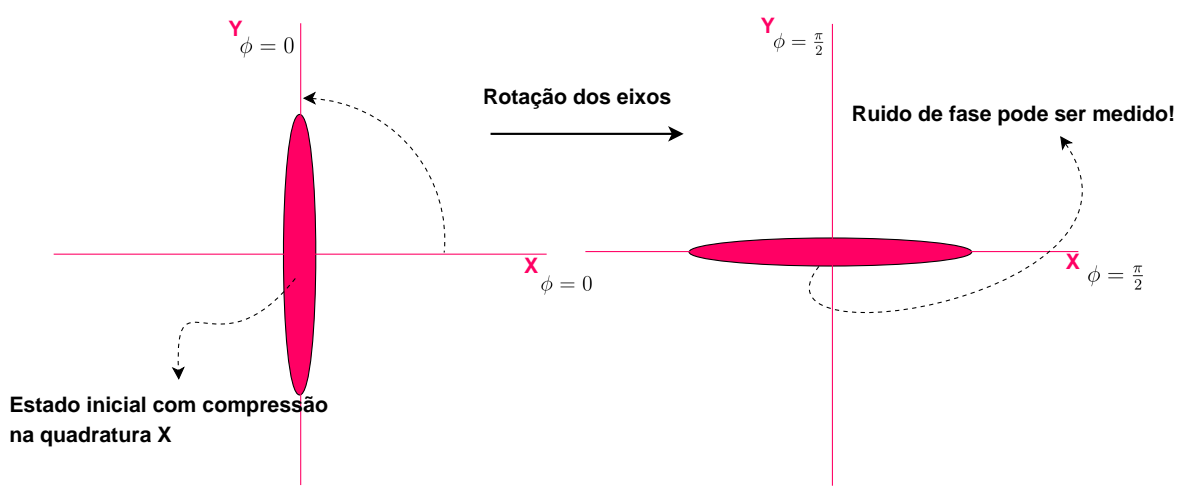

Figura 3.9: Esquema que ilustra a rotação da elipse de ruído que caracteriza um possível estado quântico do campo $\hat{a}$. Nesse estado, a compressão de ruído aparece em $\hat{X}_{\phi=0}$ e sua medida pode ser facilmente determinada experimentamente. Porém, para medir a quadratura $\hat{Y}_{\phi=0}$, é necessário girar o espaço de fase, nesse caso, de $\frac{\pi}{2}$.

informação sobre o estado de $\hat{a}$. Tal interferência provoca uma modulação de amplitude e fase no oscilador local capaz de ser sentida pelo sistema de medida através da observação do operador $\hat{X}_{\theta}$.

Apesar de clássico, o conceito de interferência [24, 25] também pode ser estendido à descrição quântica dos campos, sendo matematicamente expresso, nesse caso, como o produto tensorial entre os estados $|\beta\rangle$, do oscilador local, e $\left|\psi_{a}\right\rangle$, do campo cujas as quadraturas desejamos medir.

Tendo tudo isso em vista, vamos generalizar nossa discussão sobre a rotação da elipse e considerar o estado do campo resultante dessa interferência $|\psi\rangle$. Usaremos três espaços de fase distintos, denominados $\hat{X}-\hat{Y}$, $\hat{X}_{\theta}-\hat{Y}_{\theta}$ e $\hat{X}_{\phi}-\hat{Y}_{\phi}$, para representar, respectivamente, os seguintes estados quânticos do campo: o estado coerente distante $\beta$ da origem, o estado coerente centrado na origem e o estado comprimido $\left|\psi_{a}\right\rangle$ centrado na origem.

A Figura 3.10 ilustra a representação dos estados $\left|\psi_{a}\right\rangle$ e $|\beta\rangle$ no espaço de fase, assim como o estado $|\psi\rangle$ no espaço de fase $\hat{X}-\hat{Y}$. Nessa figura e em todas as outras que se seguem nesse texto, as elipses de ruído, sempre em rosa, representam o estado do campo a ser medido, no caso $\hat{a}$, e estão muito aumentadas comparadas ao estado coerente do oscilador local, sempre um círculo vermelho, para ajudar na visualização e entendimento.

A escolha das cores vermelho e rosa foi feita a fim de representar graficamente a proximidade das frequências óticas entre esses campos que geram o batimento. Além disso, a espessura dos traços que representam os campos, $\hat{a}$ e oscilador local, vistos na figura 3.8, é proporcional a intensidade dos feixes. Esse é um padrão gráfico que será sempre usado para representar os feixes. 


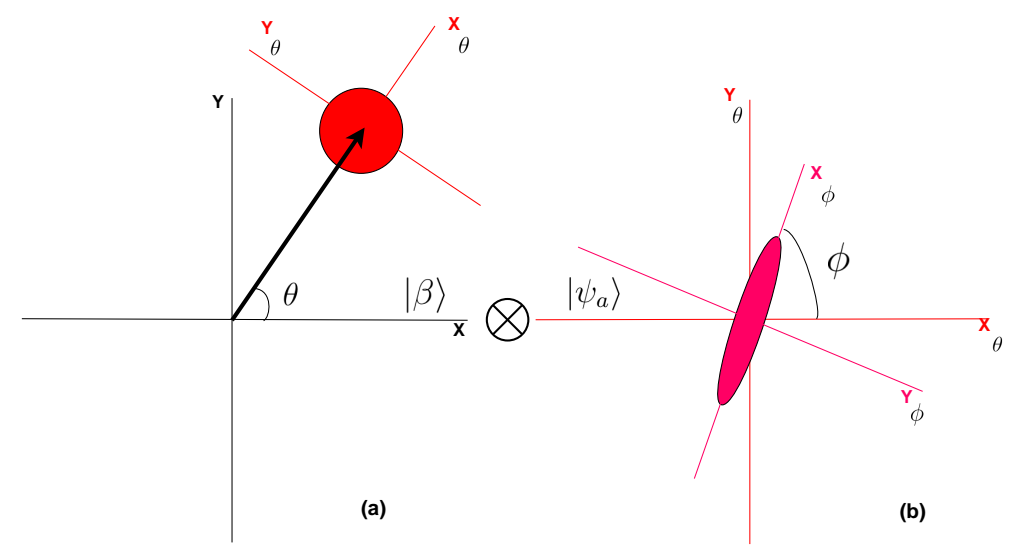

Figura 3.10: Representação da interferência entre o oscilador local e o campo a ser medido do ponto de vista quântico. Em (a), é vista a representação do estado $|\beta\rangle$ do oscilador local no espaço de fase $\hat{X}-\hat{Y}$, cuja fase é denotada por $\theta$. Essa fase define a referência $\left(\hat{X}_{\theta}\right)$ usada na medida. Em (b), é vista a representação do estado $\left|\psi_{a}\right\rangle$ do campo $\hat{a}$, cujas quadraturas pretende-se medir. Este está girado de $\phi$ em relação a $\hat{X}_{\theta}$.

Consideremos que fase do oscilador local no espaço de fase $\hat{X}-\hat{Y}$ é dada por $\theta$ e que a fase entre o oscilador local e o campo $\hat{a}$ é bem definida e dada por $\phi$ antes do processo de detecção homodina. O processo em si se inicia a partir do PZT que controla a variação das fases $\theta$ e $\phi$, conforme mostra a Figura 3.11. Essa figura exibe um possível esquema experimental para essa generalização do processo de medida. É omitido, na figura, o processo de geração de estados comprimidos por estarmos meramente interessados em como ocorre a medida.

O ângulo $\theta$ é usado para definir a quadratura $\hat{X}_{\theta}$ que será usada como referência na medição. Define-se tal referência como aquela na qual as flutuações ocorrem exatamente na direção do valor médio do oscilador local $|\beta|$, de acordo com a Figura 3.10. Todas as rotações serão escritas de agora em diante em termos dessa referência.

Pode parecer estranho adotar o eixo $\hat{X}_{\theta}$ como preferencial ao escrever as rotações da elipse de ruído. Entretanto, se recorrermos mais uma vez a uma analogia mecânica, essa necessidade ficará mais clara.

Em mecânica de corpos rígidos, existe os chamados eixos principais de inércia em torno dos quais a rotação de um corpo é simplificada e o tensor de inércia aparece diagonalizado [24]. A princípio, a rotação de um corpo rígido pode ser descrita em termos de quaisquer eixos espaciais, entretanto, quando o corpo é rotacionado ao redor de um dos seus eixos principais, tanto sua velocidade angular quanto o seu momento angular serão orientados na 


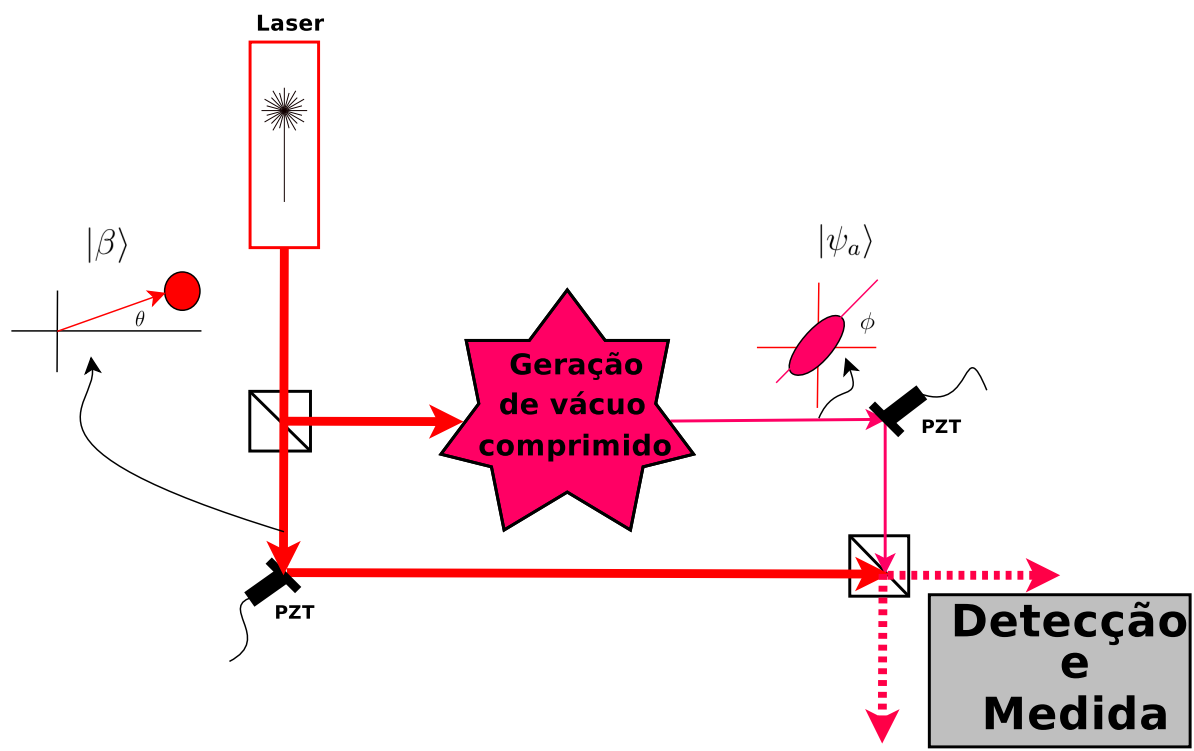

Figura 3.11: Exemplo de um esquema experimental ilustrando o processo de detecção homodina e o papel das fases $\theta$ e $\phi$. A fase $\theta$ é bem definida entre os campos porque eles são provenientes da mesma fonte. O PZT é o aparato físico responsável pela variação das fases $\theta$ e $\phi$, que resulta na rotação tanto do círculo quanto da elipse de ruído. 
direção desse eixo, o que facilita a descrição do movimento.

Voltando ao nosso caso de rotação do espaço de fase, podemos escrever a rotação tanto do ponto de vista dos eixos $\hat{X}-\hat{Y}$, quanto dos eixos $\hat{X}_{\theta}-\hat{Y}_{\theta}$. Adotaremos, como já dito, a segunda opção por simplicidade e adequação aos nossos propósitos experimentais porque, afinal, temos acesso direto às flutuações de intensidade do oscilador local. Assim, $\hat{X}_{\theta}$ funcionaria como uma espécie de "eixo principal" na rotação dos estados quânticos do campo eletromagnético.

Dessa forma, usando a matriz de rotação $\hat{\mathbf{R}}_{\phi}$ :

$$
\hat{\mathbf{R}}_{\phi}=\left[\begin{array}{cc}
\cos \phi & -\operatorname{sen} \phi \\
\operatorname{sen} \phi & \cos \phi
\end{array}\right]
$$

escreve-se $\hat{X}_{\phi}$ e $\hat{Y}_{\phi}$ como sendo a rotação dos eixos $\hat{X}_{\theta}$ e $\hat{Y}_{\theta}$ de um ângulo $\phi$, conforme as equações (3.28) e (3.29):

$$
\begin{aligned}
& \hat{X}_{\theta}=\hat{X}_{\phi} \cos \phi-\hat{Y}_{\phi} \operatorname{sen} \phi \\
& \hat{Y}_{\theta}=\hat{X}_{\phi} \operatorname{sen} \phi+\hat{Y}_{\phi} \cos \phi
\end{aligned}
$$

Como o acesso experimental é feito através da medida da intensidade do campo resultante da interferência, associada ao operador quântico $\hat{X}_{\theta}$, calculamos a variância desse operador. De posse da equação (3.28) e, usando as mesmas hipóteses usadas anteriormente no início dessa seção ${ }^{10}$, obtemos:

$$
\left\langle\psi\left|\Delta^{2} \hat{X}_{\theta}\right| \psi\right\rangle=\cos ^{2} \phi\left\langle\psi\left|\Delta^{2} \hat{X}_{\phi}\right| \psi\right\rangle+\operatorname{sen}^{2} \phi\left\langle\psi\left|\Delta^{2} \hat{Y}_{\phi}\right| \psi\right\rangle-2 \cos \phi \operatorname{sen} \phi \operatorname{Cov}\left(\hat{X}_{\phi} \hat{Y}_{\phi}\right)
$$

Da equação (3.27), concluimos que:

$$
\operatorname{Var}\left(i_{c}-i_{d}\right)=\beta^{2}\left\langle\psi_{a}\left|\Delta^{2} \hat{X}_{\theta}\right| \psi_{a}\right\rangle
$$

Assim, a variância da subtração das fotocorrentes é a combinação de variâncias de ambas as quadraturas com pesos que dependem do ângulo $\phi$ e de um termo $\operatorname{Cov}\left(\hat{X}_{\phi} \hat{Y}_{\phi}\right)$, até então não definido. Esse termo é a covariância entre as grandezas $\hat{X}_{\phi}$ e $\hat{Y}_{\phi}$ e diz respeito à correlação quântica entre as mesmas.

Se $\operatorname{Cov}\left(\hat{X}_{\phi} \hat{Y}_{\phi}\right)=0$, então essas quadraturas são ditas independentes ou não correlacionadas, caso contrário, possuem correlações. Além disso, para ter acesso às quadraturas separadamente, confimando o que foi visto no caso

\footnotetext{
${ }^{10} \mathrm{Na}$ verdade, usou-se mais uma hipótese não mencionada aqui. Supomos, nesse cálculo, que os estados quânticos envolvidos são gaussianos, ou seja, estados que obedecem uma estatística gaussiana. Dessa forma, podemos usar que $\left\langle\hat{X}_{\phi} \hat{Y}_{\phi}\right\rangle=\frac{\left\langle\hat{X}_{\phi} \hat{Y}_{\phi}+\hat{Y}_{\phi} \hat{X}_{\phi}\right\rangle}{2}$, segundo visto na referência [26]. Sem o uso de tal hipótese, aparece o termo imaginário isen $\phi \cos \phi$ que torna a variância da subtração das correntes imaginária.
} 
particular em que os eixos $\hat{X}_{\phi}-\hat{Y}_{\phi}$ estavam alinhados aos eixos $\hat{X}_{\theta}-\hat{Y}_{\theta}$, basta fazer $\phi=0$ para obter a quadratura relativa à intensidade ou $\phi=\frac{\pi}{2}$ para obter a fase.

Da mesma forma como em probabilidade [27], a covariância é definida quanticamente como:

$$
\operatorname{Cov}\left(\hat{X}_{\phi} \hat{Y}_{\phi}\right)=\left\langle\psi\left|\left(\hat{X}_{\phi}-\left\langle\psi\left|\hat{X}_{\phi}\right| \psi\right\rangle\right)\left(\hat{Y}_{\phi}-\left\langle\psi\left|\hat{Y}_{\phi}\right| \psi\right\rangle\right)\right| \psi\right\rangle
$$

A equação (3.32) pode ser igualmente simplificada como em probabilidade, em que a covariância refere-se a variáveis aleatórias, de acordo com:

$$
\operatorname{Cov}\left(\hat{X}_{\phi} \hat{Y}_{\phi}\right)=\left\langle\psi\left|\hat{X}_{\phi} \hat{Y}_{\phi}\right| \psi\right\rangle-\left\langle\psi\left|\hat{X}_{\phi}\right| \psi\right\rangle\left\langle\psi\left|\hat{Y}_{\phi}\right| \psi\right\rangle
$$

Um ponto importante a se considerar é que, se adotamos $\hat{X}$ como referência na medida, $\hat{X}_{\phi}-\hat{Y}_{\phi}$ são escritos como a rotação e a translação dos eixos $\hat{X}-\hat{Y}$, exatamente como vistos na parte (c) da figura 3.10 e, matematicamente, expressos como:

$$
\begin{aligned}
& \hat{X}=\hat{X}_{\phi} \cos \phi-\hat{Y}_{\phi} \operatorname{sen} \phi+|\beta| \cos \theta \\
& \hat{Y}=\hat{X}_{\phi} \operatorname{sen} \phi+\hat{Y}_{\phi} \operatorname{sen} \phi+|\beta| \operatorname{sen} \theta
\end{aligned}
$$

Calculando-se a variância de $\hat{X}$, dada a equação (3.34), obtemos exatamente o mesmo resultado expresso na equação (3.30). Como esperado, a $\operatorname{Var}\left(i_{c}-i_{d}\right)$, que é a grandeza com realidade física, independe da escolha da quadratura ( $\hat{X}$ ou $\left.\hat{X}_{\theta}\right)$ usada como referência.

A rotação da elipse de ruído promovida pela detecção homodina, apesar de descrita nessa seção para feixes pouco intensos como vácuo comprimido, também pode ser realizada na detecção de feixes intensos, desde que esses feixes tenham intensidades muito menores que o oscilador local utilizado.

Entretanto, conforme veremos mais adiante, no caso de feixes intensos, o uso de cavidades óticas vazias para implementar a rotação da elipse mostrase muito mais vantajosa. Esse tipo de detecção é conhecida como detecção auto-homodina [8] por já ter incluso, no próprio feixe a ser medido, o oscilador local.

Tal técnica vem sendo amplamente utilizada e investigada no nosso laboratório nos últimos anos e tem se mostrado a mais indicada na reconstrução do estado quântico, por ser mais completa que a detecção homodina, como visto nos últimos trabalhos do grupo [28, 29].

Antes de descrever a técnica de detecção auto-homodina, falaremos sobre a calibração do sistema de medida e, em seguida, teceremos alguns comentários referente à medição das fotocorrentes e a caracterização do seu ruído, que, até esse ponto, foi feita através da variância, sem nenhuma discussão mais aprofundada. Além disso, abordaremos também sobre um jargão 
muito empregado em engenharia de telecomunicações no contexto de transmissão de informação através da modulação de amplitude e fase de um sinal elétrico. Faremos um paralelo entre o contexto elétrico e o ótico e, então, passaremos a adotar esse mesmo jargão no nosso caso de transmissão de informação quântica.

\subsection{A calibração do "shot noise"}

O ato de medir uma grandeza física, ou atribuir um número a essa grandeza, implica em uma comparação com alguma outra grandeza semelhante usada como referência ou escala. Na medida do ruído quântico, associado a uma dada quadratura do campo eletromagnético, não é diferente. A escala utilizada nesse caso é denominada na literatura de shot noise ou ruído quântico padrão, que é o ruído produzido no sistema de medida quando o estado $\left|\psi_{a}\right\rangle$ é o estado de vácuo $|0\rangle$.

A princípio, qualquer estado $\left|\psi_{a}\right\rangle$ do campo $\hat{a}$, incidente no BS da figura 3.8, poderia servir na calibração do ruído quântico. Entretanto, é imprescindível que o estado escolhido para tal tenha as menores flutuações de intensidade e fase possíveis. Sabemos do capítulo 2 que o estado coerente é o estado quântico do campo satisfazem essa condição.

O estado quântico do campo produzido em um laser comercial, por exemplo, é aproximadamente coerente, mas não é o ideal porque existe excesso de ruído de fase e amplitude. Assim, a utilização do estado de vácuo é o mais apropriado para a calibração porque ele estabelece um limite mínimo de flutuação que pode ser utilizado para comparar com outros ruídos gerados por campos cujas quadraturas pretende-se medir.

A figura 3.12 ilustra um exemplo de esquema usado no laboratório para medida do shot noise. Como a homodinagem é feita com o vácuo, somente há um feixe incidindo no BS. Os feixes resultantes são detectados e as fotocorrentes geradas somadas/subtraídas. O tratamento matemático da detecção homodina, considerando o estado do oscilador local perfeitamente coerente e o caso especial em que $\left|\psi_{a}\right\rangle$ é o estado de vácuo, é realizado a seguir.

Antes de considerar essa situação, entretanto, é preciso realizar o mesmo procedimento anterior para calcular a variância da soma das fotocorrentes $i_{c}+i_{d}$. O cálculo da média resulta, assumindo a hipótese que o estado $|\psi\rangle$ é normalizado,

$$
\overline{i_{c}+i_{d}}=\left\langle\psi\left|\hat{a}^{\dagger} \hat{a}+\hat{b}^{\dagger} e^{-i \phi} \hat{b} e^{i \phi}\right| \psi\right\rangle=\left\langle\psi\left|\hat{a}^{\dagger} \hat{a}\right| \psi\right\rangle+\beta^{2}
$$

Em seguida, determina-se a média de $\left(i_{c}+i_{d}\right)^{2}$ :

$$
\overline{\left(i_{c}+i_{d}\right)^{2}}=\left\langle\psi\left|\left(\hat{a}^{\dagger} \hat{a}\right)^{2}+\hat{a}^{\dagger} \hat{a} \hat{b}^{\dagger} \hat{b}+\hat{b}^{\dagger} \hat{b} \hat{a}^{\dagger} \hat{a}+\left(\hat{b}^{\dagger} \hat{b}\right)^{2}\right| \psi\right\rangle
$$




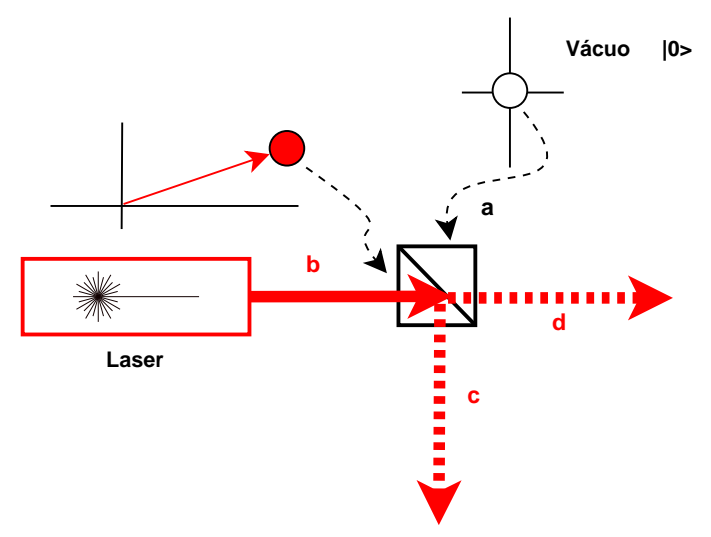

Figura 3.12: Esquema experimental utilizado na calibração das medidas. O laser comercial gera um campo cujo estado quântico pode ser aproximado como sendo um estado coerente. Esse campo é usado tanto na geração de estados comprimidos como oscilador local, visto na figura 3.11. Antes do processo de medida, é necessário estabelecer o limite mínimo de flutuações de ruído que será utilizado como base de comparação para os outros ruídos. Isso é feito simplesmente através da passagem de somente o feixe do laser através do BS e sua detecção pelo sistema de medida.

Colocando-se o operador $\left(\hat{b}^{\dagger} \hat{b}\right)^{2}$ na ordem normal ${ }^{11}$, a equação (3.37) assume a forma:

$$
\overline{\left(i_{c}+i_{d}\right)^{2}}=\left\langle\psi\left|\left(\hat{a}^{\dagger} \hat{a}\right)^{2}\right| \psi\right\rangle+2 \beta^{2}\left\langle\psi_{a}\left|\hat{a}^{\dagger} \hat{a}\right| \psi_{a}\right\rangle+\left\langle\psi \mid \hat{b}^{\dagger} \hat{b} \psi\right\rangle+\left\langle\psi\left|\hat{b}^{\dagger} \hat{b}^{\dagger} \hat{b} \hat{b}\right| \psi\right\rangle
$$

O que resulta em:

$$
\overline{\left(i_{c}+i_{d}\right)^{2}}=\left\langle\psi\left|\left(\hat{a}^{\dagger} \hat{a}\right)^{2}\right| \psi\right\rangle+2 \beta^{2}\left\langle\psi_{a}\left|\hat{a}^{\dagger} \hat{a}\right| \psi_{a}\right\rangle+\beta^{2}+\beta^{4}
$$

A variância da soma das fotocorrentes $\left(i_{c}+i_{d}\right)$ é calculada de posse das equações (3.36) e (3.39), resultando em:

$$
\operatorname{Var}\left(i_{c}+i_{d}\right)=\left\langle\psi\left|\Delta^{2}\left(\hat{a}^{\dagger} \hat{a}\right)\right| \psi\right\rangle+\beta^{2}
$$

Como, por hipótese, o estado do campo â é o estado de vácuo e a variância do operador $\hat{a}^{\dagger} \hat{a}$ para esse estado é igual a zero, a variância $\operatorname{Var}\left(i_{c}+i_{d}\right)$ é finalmente fornecida por:

$$
\operatorname{Var}\left(i_{c}+i_{d}\right)=\beta^{2}
$$

\footnotetext{
${ }^{11}$ Em um operador na ordem normal, os operadores criação estão à frente dos operadores de aniquilação.
} 
Nesse caso particular, o estado do sistema é $|\psi\rangle=|\beta\rangle \otimes|0\rangle$, onde $|\beta\rangle$ é um estado coerente. A figura 3.13 mostra essa situação em que a interferência entre campos coerentes gera um outro campo cujo estado $|\psi\rangle$ é igualmente coerente. Independente da variação das fases $\phi, \theta$, o ruído de fase e amplitude desse estado é sempre o mesmo, devido à intrínseca simetria inerente ao estado coerente.

Idealmente, o estado do oscilador local é coerente e, no espaço de fase, ele se sobrepõe ao estado de vácuo, produzindo um estado com mesmo ruído de fase e amplitude. Entretanto, como já apontamos, lasers comerciais sempre apresentam excesso de ruído, o que, logicamente, afeta as medidas, aumentando a escala de flutuação mínima. Dessa forma, em termos reais, o estado do oscilador é como ilustrado na parte $(a)$ da figura 3.14.

Ainda na mesma figura, vemos um exemplo de estado comprimido em fase (em rosa), cujas quadraturas pretende-se medir. A medição ocorre através da comparação do ruído projetado em $\hat{X}_{\theta}$ com o ruído dado pelo shot noise. Na parte $(b)$ da figura, $\phi=0$, tem-se a projeção do ruído de amplitude projetada e, na parte (c), tem-se $\phi=\frac{\pi}{2}$ e a projeção do ruído de fase.

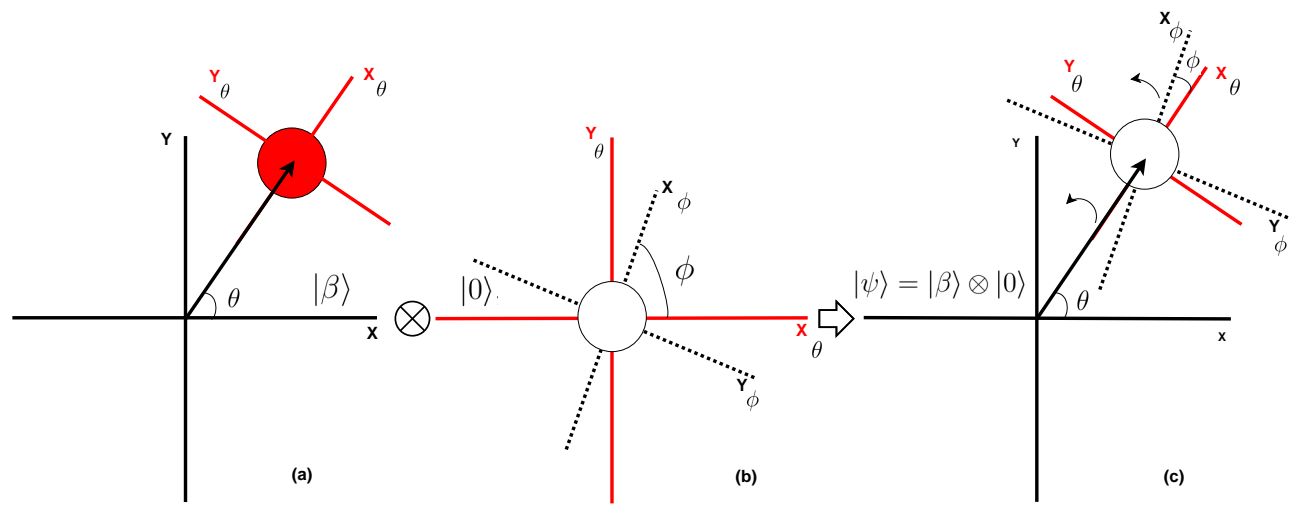

Figura 3.13: Homodinagem com o vácuo no espaço de fase. A interferência do oscilador local, cujo estado é coerente idealmente, e o vácuo, no BS da figura 3.12, gera um campo cujo o estado produz um ruído quântico que é sempre o mesmo independente da variação das fases $\phi, \theta$. Esse ruído é usado como escala de flutuação mínima. Qualquer estado do campo eletromagnético medido é comparado a essa escala.

Aplicando-se a equação (3.27) ao estado de vácuo e, recordando-se que a variância das quadraturas do estado de vácuo é igual a 1, observamos que a flutuação da subtração das fotocorrentes varia de forma linear com a intensidade do oscilador local, $\beta^{2}$ :

$$
\operatorname{Var}\left(i_{c}-i_{d}\right)=\beta^{2}\left\langle 0\left|\Delta^{2} \hat{X}_{\phi}\right| 0\right\rangle=\beta^{2}
$$




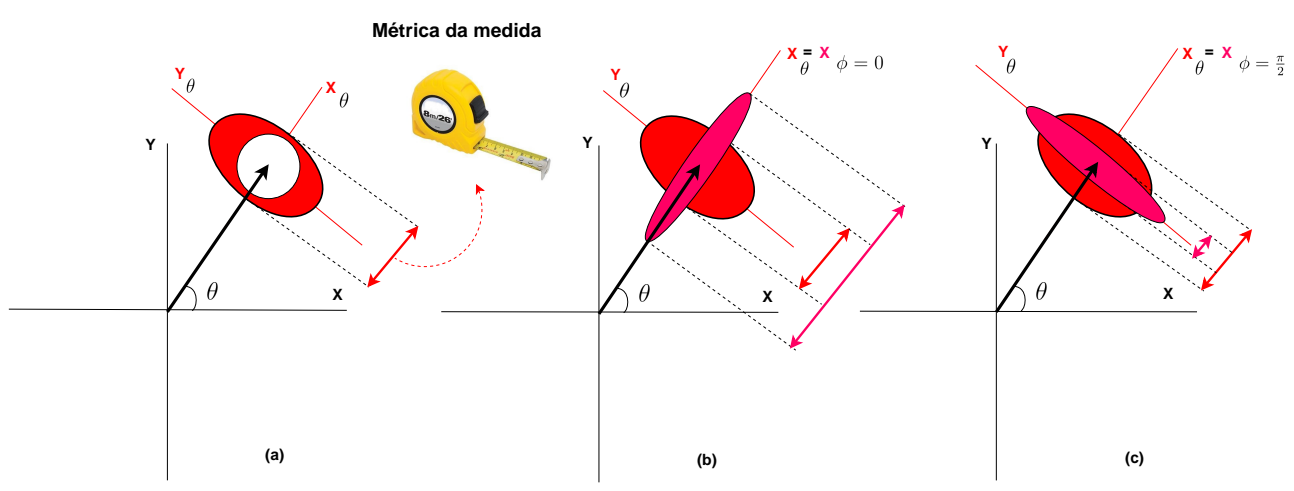

Figura 3.14: Em (a), é vista a interferência entre o estado de vácuo(círculo branco) e o estado com excesso de ruído de fase e amplitude oferecido pelo laser comercial e usado como oscilador local. Essa interferência nos oferece uma escala, shot noise, para a medição de ruídos. Em (b), temos um exemplo da medida da quadratura intensidade do estado em rosa. Comparando-se com a escala de medida, é visto a representação de excesso de ruído. Em (c), a elipse é rodada e temos acesso a medida da fase. Como visto, comparado à escala, há compressão de ruído.

Além disso, usando-se a equação (3.36) para o estado de vácuo e dada a equação (3.42), temos que:

$$
\operatorname{Var}\left(i_{c}-i_{d}\right)=\overline{i_{c}+i_{d}}=\beta^{2}
$$

Esse é o mais importante resultado eletrônico conhecido para o ruído de shot noise que diz respeito unicamente às flutuações produzidas pela intensidade da luz ao incidir no fotodetector. Tal equação é sempre válida quando os elétrons que atingem o fotodetector são gerados estatisticamente de forma independente [18].

Outra forma de interpretar a equação (3.43) é dizer que, no caso do shot noise, o valor médio de $i_{c}+i_{d}$, referente à medida da intensidade total da luz, que pode ser realizada por somente um detector, reflete as flutuações da subtração das fotocorrentes $i_{c}-i_{d}$, quando a medida é feita por dois detectores [5].

Outra característica óbvia do shot noise vem diretamente das equações (3.40) e (3.42):

$$
\frac{\operatorname{Var}\left(i_{c}+i_{d}\right)}{\operatorname{Var}\left(i_{c}-i_{d}\right)}=1
$$

Na prática experimental, a manipulação eletrônica do ruído gerado pelas fotocorrentes, sinais HF, ocorre por meio de etapas de amplificação e filtragem. Esses processos fazem com que tanto a equação (3.43) como a (3.44) sejam 
proporcional a algum fator de ganho $k_{\text {eletro }}$ que depende da eletrônica envolvida na medida. Tal fator foi sempre desconsiderado por praticidade, porém é determinado no processo de calibração das medidas.

No caso das nossas medidas, usamos um sistema eletrônico especial, chamado de demodulação, capaz de extrair a informação quântica presente no ruído do sinal eletrônico e enviá-lo a um computador. Devido ao processo de amplificação de transimpedância que ocorre no circuito do detector, como vimos, esses sinais são medidos em volts através da interface analógico-digital instalada no computador. Todos os cálculos de variância da soma ou subtração das fotocorrentes são realizados por meio de softwares.

Cada sistema de medida depende fortemente da sua eletrônica, às vezes, até em um mesmo laboratório pode-se ter circuitos eletrônicos com ganhos ligeramente distintos que fornecem valores completamente diferentes para a medida bruta do ruído da quadratura de um campo em um mesmo estado quântico.

Dessa forma, é necessário, mais uma vez, o uso de uma unidade de medida que compare a medida do ruído de um dado estado quântico com o ruído medido para o shot noise e elimine essa dependência da eletrônica. A unidade mais conveniente, conforme previamente discutido, é o decibel vastamente empregada em engenharia de telecomunicações onde a utilização de amplificações e filtros eletrônicos, como descrito anteriormente, são frequentes no tratamento de sinais elétricos.

No nosso contexto quântico, toda medida de compressão ou excesso de ruído em relação à medida do shot noise será, de agora em diante, expressa em decibel, redefinido ${ }^{12}$ como:

$$
\operatorname{Medida}(d B)=10 \log \left(\frac{\text { medida }}{\text { shot noise }}\right)
$$

Se traçarmos um paralelo com o que foi visto sobre o ganho eletrônico no Apêndice A, perceberemos que o excesso/compressão de ruído eletrônico, no caso de uma medida de natureza quântica, funciona como uma espécie de ganho quântico do sistema físico medido.

No capítulo 6 será descrito todo o procedimento experimental que adotamos para a calibração do shot noise usada nas nossas medidas.

\footnotetext{
${ }^{12}$ Para as medidas de potência e intensidade é usada a definição (3.45), porém para medidas como tensão ou corrente elétrica usa-se a definição (A.30).
} 


\subsection{Considerações sobre medidas e teoria de flutuação}

Até agora só falamos da interferência dos campos e os estados quânticos que os representam no espaço de fase complexo. Nessa seção, focaremos nos aspectos relativos às medidas das fotocorrentes, geradas no processo de detecção, que carregam toda a informação quântica e no tratamento estatístico dado a elas.

A maior parte da teoria abordada aqui sobre as flutuações de grandezas físicas, descrita por processos estocásticos, foi baseada na referência [23]. O intuito dessa seção não é tratar de forma matematicamente rigorosa conceitos da probabilidade, mas fornecer uma visão geral da teoria que é aplicada no tratamento das nossas medidas no laboratório.

A atribuição de um valor real a uma determinada grandeza física nunca se realiza de maneira precisa por causa das limitações intrínsecas ao próprio ato de medir ou ao ato de comparar essa grandeza com outra semelhante, considerada como padrão da medida. Assim, para toda grandeza medida existe uma incerteza associada que torna o valor dela não determinístico e sempre interpretado à luz da teoria de probabilidade.

Existe determinadas grandezas físicas que, em certas condições experimentais bem específicas, não variam no tempo ${ }^{13}$. A medida de uma grandeza física desse tipo é considerada, no jargão da teoria de probabilidade, como uma variável aleatória, que denotaremos por $x$.

O conjunto de todos os possíveis resultados que a medida dessa grandeza pode assumir, ou possíveis valores de $x$, é chamado de espaço amostral, em termos estatísticos, ou ensemble, na linguagem comumente usada em física estatística. A medida ${ }^{14}$ do quão provável é o valor de uma dada grandeza física medida, $x$, é fornecida pela sua função de probabilidade, $P(x)$, que, em geral, é determinada experimentalmente em termos frequentistas, ou seja, pela repetição da medida, nas mesmas condições experimentais, $n$ vezes.

Entretanto, a maioria das grandezas físicas submetidas a um processo de medição, na verdade, são grandezas que variam no tempo e suas medidas, em termos probabilísticos, são ditas processos aleatórios ou estocásticos ${ }^{15}$.

Um processo estocástico contínuo, $x(t)$, é um conjunto contínuo de variáveis aleatórias $x_{1}\left(t_{1}\right), x_{2}\left(t_{2}\right), x_{3}\left(t_{3}\right), \ldots$ indexado pelo parâmetro temporal $t$, ou,

\footnotetext{
${ }^{13}$ Como, por exemplo, a massa e o volume de um corpo em condições macroscópicas.

${ }^{14}$ Medida no sentido de associar um peso probabilístico a ocorrência de dada medida física.

${ }^{15}$ Quando uma variável aleatória varia em relação a um parâmetro e, se esse parâmetro é o tempo, então é dito ser estocástico.
} 
em outras palavras, é um processo tal que, para cada tempo $t_{i}$, temos uma variável aleatória $x_{i}$ associada. Toda flutuação temporal de qualquer grandeza física medida é considerada, estatisticamente, como um processo estocástico.

Um exemplo muito comum de uma medida que apresenta flutuações no tempo é a medida da tensão elétrica com o uso de um osciloscópio. É sabido que a corrente elétrica ao atravessar um resistor a uma dada temperatura, em um circuito elétrico qualquer, é afetada por flutuações térmicas proporcionais à essa temperatura e à constante de Boltzmann e inversamente proporcional à impedância do circuito [18], o que altera continuamente o valor medido da tensão.

Esse tipo de flutuação detectado experimentalmente é conhecido como ruído térmico ou de Johnson e junto a outros tipos de ruídos, como o "dark noise" e o ruído devido a todos os processos de amplificação eletrônica, são responsáveis por um ruído denominado genericamente de ruído eletrônico. Veremos, mais adiante, que tal ruído é descontado das nossas medidas de ruído quântico.

Supomos que tal processo estocástico é sempre medido por um dispositivo capaz de realizar $n$ medidas de cada variável aleatória $x_{i}\left(t_{i}\right)$ em um dado instante $t_{i}$ de tempo. Obviamente, essa é uma suposição que assumimos, pois o ato de medir uma grandeza física não é instantâneo, ou seja, em termos práticos, é fisicamente impossível medir uma grandeza repetidas vezes em um intervalo de tempo $\Delta t=0$. Porém, o nosso dispositivo de medida via computador realiza 1000 medidas de uma dada variável $x_{i}$, cada medida é feita em um curto intervalo de tempo $\Delta t=1,6 \mu \mathrm{s}$ e, dessa forma, a nossa aproximação será tal que $x_{i}\left(t_{i}+\Delta t\right) \approx x_{i}\left(t_{i}\right)$.

A janela temporal total de aquisição das nossas medidas é de $740 \mathrm{~ms}$ e, durante essa janela, temos, como dito anteriormente, a amostragem de 1000 repetições de cada variável $x_{i}$ do processo estocástico $x(t)$ que, no nosso caso, são as flutuações de tensão resultantes da detecção e aquisição das fotocorrentes geradas pela exposição dos detectores à luz.

\subsubsection{Funções de probabilidade dos processos estocásticos}

Vejamos, de maneira resumida, a teoria probabilística por trás da medida de grandezas físicas que flutuam no tempo, $x(t)$. Considere que a cada instante de tempo $t$, existe uma função $P(x, t)$ de probabilidade que governa estatisticamente a variável $x$, de forma que, para calcular a média $\bar{x}$, temos:

$$
\bar{x}=\int x P(x, t) d x
$$


Como nem sempre temos conhecimento das funções $P(x, t)$ que governam o processo estocástico $x(t)$ a cada instante de tempo, podemos experimentalmente repetir a medida de $x$ várias vezes tal que a média pode ser calculada, alternativamente, como:

$$
\bar{x}=\lim _{n \rightarrow \infty} \frac{\sum_{i=1}^{n} x^{(i)}(t)}{n}
$$

em que $x^{(i)}(t)$ representa cada realização da medida de $x(t)$ e o conjunto $\left\{x^{(i)}(t)\right\}$ de todas as repetições experimentais realizadas é conhecido, da mesma maneira como na situação de uma única variável aleatória, como ensemble de $x(t)$. Apesar de não considerar, em termos experimentais, as funções de probabilidade que governam certo processo estocástico, faremos sempre uso delas aqui apenas para explicar a teoria das flutuações.

Para ilustrar os conceitos de processo estocástico e seu ensemble, temos a figura 3.15. A parte (a) da figura fornece uma visão esquemática de um exemplo de medida de uma grandeza física $x(t)$ variável no tempo, exemplo de um processo estocástico. A parte (b) da mesma figura mostra o ensemble de medidas dessa grandeza física. Cada variável $x_{i}\left(t_{i}\right)$ pertencente ao processo foi medida $N$ vezes, o que implica dizer que o processo também foi repetido $N$ vezes. Essas são duas visões completamente equivalentes do ensemble de medidas de $x(t)$.

As funções $P(x, t)$ não contém nenhuma informação sobre possíveis correlações entre as variáveis aleatórias, em diferentes intervalos de tempo, que fazem parte do processo estocástico de $x(t)$. Para isso, é necessário recorrer as chamadas funções de autocorrelações definidas através das funções de probabilidade conjunta.

Consideremos duas variáveis aleatórias $x_{1}$ e $x_{2}$ pertencentes ao processo estocástico $x(t)$ dadas nos instantes $t_{1}$ e $t_{2}$ respectivamente. Com a probabilidade conjunta delas, fornecida por $P_{2}\left(x_{2}\left(t_{2}\right) x_{1}\left(t_{1}\right)\right)$, é possível calcular sua função autocorrelação como:

$$
C\left(t_{1}, t_{2}\right)=\overline{x_{1}\left(t_{1}\right) x_{2}\left(t_{2}\right)}=\int x_{1} x_{2} P_{2}\left(x_{2}\left(t_{2}\right) x_{1}\left(t_{1}\right)\right) d x_{1} d x_{2}
$$

Em termos de ensemble, essa função é obtida por:

$$
\overline{x_{1}\left(t_{1}\right) x_{2}\left(t_{2}\right)}=\lim _{n \rightarrow \infty} \frac{\sum_{i=1}^{n} x_{1}^{(i)}\left(t_{1}\right) x_{2}^{(i)}\left(t_{2}\right)}{n}
$$

A informação dada pela função de correlação é de fundamental importância para se entender a dinâmica do processo estocástico, tal como saber o grau 


\section{Medida de $\mathrm{x}$ : processo estocástico}
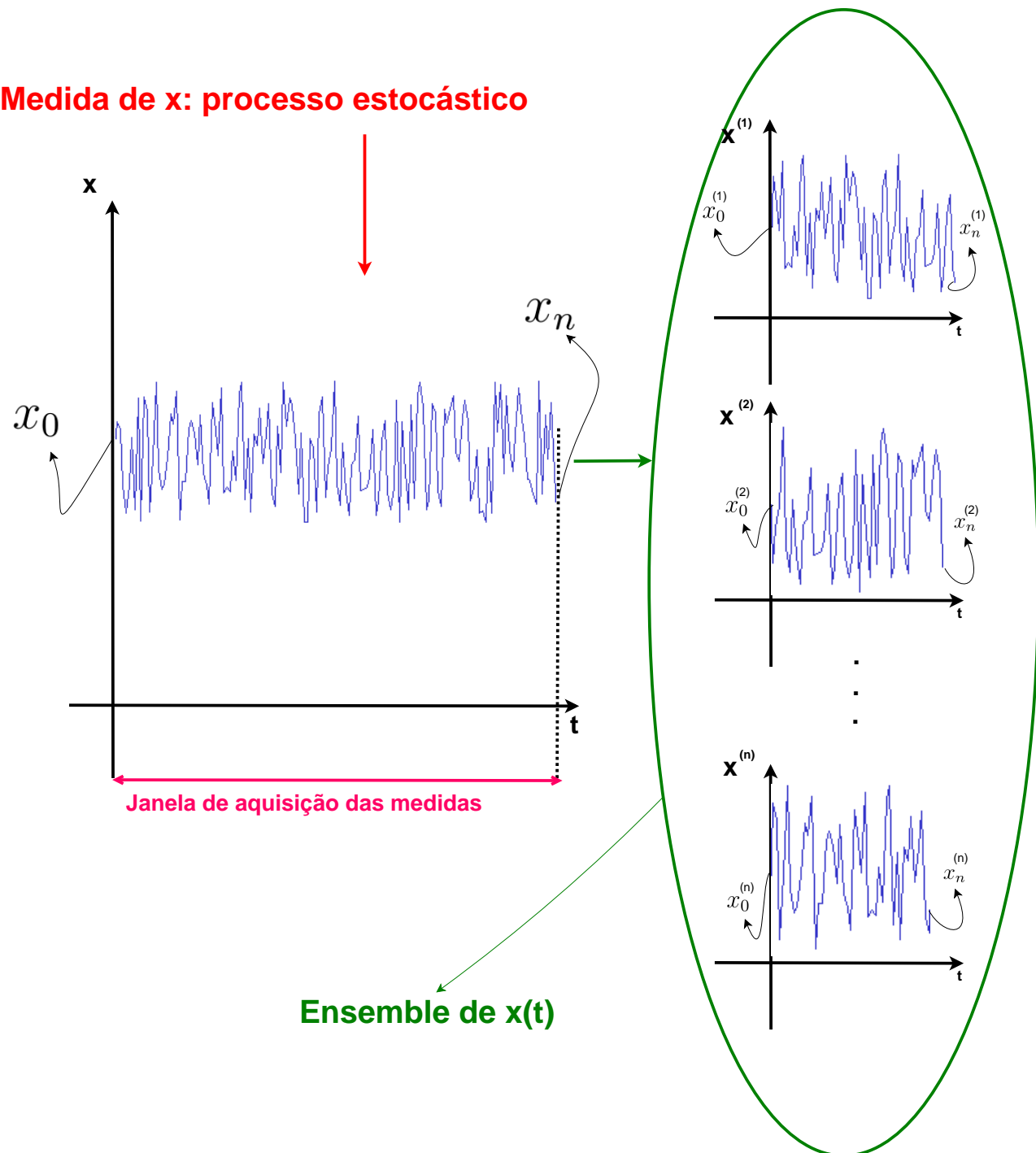

(a)

(b)

Figura 3.15: Figura adaptada da refrência [23]. Na parte (a), é visto um exemplo de uma medida de uma grandeza física que varia no tempo. A flutuação temporal da medida dessa grandeza é um processo estocástico, o que significa que a cada tempo $t_{1}, t_{2}, t_{3} \ldots$ existe uma variável aleatória $x_{1}, x_{2}, x_{3}, \ldots$ associada. Na parte (b), é visto o ensemble de medidas do processo estocástico $x(t)$. Cada variável aleatória $x_{i}\left(t_{i}\right)$ do processo foi medida $N$ vezes. 
de semelhança entre os sinais ou quanto tempo dura uma correlação. Ela pode ser facilmente generalizada para $n$ variáveis aleatórias do processo $x(t)$ :

$$
C\left(t_{1}, t_{2} \ldots t_{n}\right)=\int x_{1} x_{2} \ldots x_{n} P_{n}\left(x_{n}\left(t_{n}\right), \ldots, x_{2}\left(t_{2}\right), x_{1}\left(t_{1}\right)\right) d x_{1} d x_{2} \ldots d x_{n}
$$

\subsubsection{Processos estocásticos estacionários}

Um interessante tipo de processo estocástico bastante encontrado na Física é conhecido como estacionário cuja a forma de flutuação não muda com o tempo. Definido-se de forma mais precisa esse conceito, é um processo no qual as funções de probabilidade $P_{1}, P_{2} \ldots, P_{n}$ que governam as suas flutuações são invariantes por translação temporal. Isso significa que, para um tempo $T$ qualquer, teremos sempre:

$$
\begin{gathered}
P_{1}\left(x_{1}\left(t_{1}+T\right)\right)=P_{1}\left(x_{1}\left(t_{1}\right)\right) \\
P_{2}\left(x_{2}\left(t_{2}+T\right), x_{1}\left(t_{1}+T\right)\right)=P_{n}\left(x_{2}\left(t_{2}\right), x_{1}\left(t_{1}\right)\right) \\
\cdot \\
\cdot \\
\cdot \\
P_{n}\left(x_{n}\left(t_{n}+T\right), \ldots, x_{2}\left(t_{2}+T\right), x_{1}\left(t_{1}+T\right)\right)=P_{n}\left(x_{n}\left(t_{n}\right), \ldots, x_{2}\left(t_{2}\right), x_{1}\left(t_{1}\right)\right)
\end{gathered}
$$

Dessa maneira, os cálculos das médias das variáveis aleatórias, bem como as suas funções de autocorrelação são invariantes por translação temporal, como por exemplo:

$$
\begin{gathered}
\overline{x_{1}\left(t_{1}+T\right)}=\overline{x_{1}\left(t_{1}\right)} \\
\overline{x_{1}\left(t_{1}+T\right) x_{2}\left(t_{2}+T\right)}=\overline{x_{1}\left(t_{1}\right) x_{2}\left(t_{2}\right)}
\end{gathered}
$$

Outra forma de encarar a invariância temporal dos processos estacionários é verificar que as funções de autocorrelação dependem somente da diferença entre os tempos $t_{2}-t_{1}$, no caso em que $T=t-t_{1}$ ou $T=t-t_{2}$ :

$$
C\left(t_{1}, t_{2}\right)=C\left(t_{1}+T, t_{2}+T\right)=C\left(t, t+t_{2}-t_{1}\right)=C\left(t+t_{1}-t_{2}, t\right)
$$

As fotocorrentes geradas no nosso experimento sempre obedecem a condição de estacionaridade. A razão disso é que os estados quânticos $|\psi\rangle$ (ou misturas estatísticas $\rho$ ) produzidos no laboratório não variam, por hipótese, durante a realização da medida, garantindo, assim, a invariância temporal das funções de probabilidade envolvidas. 
Outra hipótese que assumimos e não exploraremos teoricamente nessa seção é que, além de estacionários, os processos estocásticos tratados no laboratório também são ditos ergódicos. Grosso modo, ergodicidade significa que toda a informação estatística é adquirida com a realização do processo uma única vez em uma dada janela de tempo, o que implica dizer que nenhuma informação estatística a mais será obtida da observação do mesmo processo diversas vezes.

Apesar de tratarmos, até o momento, de processos estocásticos reais, $x(t)$, a mesma teoria pode ser aplicada a processos complexos. Tais processos são resultados da combinação de dois processos estocásticos reais com uma relação de fase bem estabelecida entre eles. Chamaremos de $\mathbf{x}(t)$ esses processos complexos dados por:

$$
\mathbf{x}(t)=x_{R}+i x_{I},
$$

em que $x_{R}$ é um dos processos, considerado como a parte real do processo complexo $\mathbf{x}(t)$, e $x_{I}$ o outro processo considerado como a parte imaginária.

Para tornar a discussão mais geral, trataremos sempre de processos complexos. Nesse tipo de processo, a função de autocorrelação $C$ passa a ser definida como, por exemplo, para $C\left(t_{1}, t_{2}\right)$ :

$$
C\left(t_{1}, t_{2}\right)=\overline{\mathbf{x}_{1}^{*}\left(t_{1}\right) \mathbf{x}_{2}\left(t_{2}\right)}=\int \mathbf{x}_{1}^{*} \mathbf{x}_{2} P_{2} d^{2} \mathbf{x}_{1} d^{2} \mathbf{x}_{2}
$$

No caso do processo $\mathbf{x}(t)$ ser estacionário, temos que:

$$
C\left(t_{1}, t_{2}\right)=\overline{\mathbf{x}^{*}(t) \mathbf{x}\left(t+t_{2}-t_{1}\right)}=\overline{\mathbf{x}^{*}\left(t+t_{1}-t_{2}\right) \mathbf{x}(t)}
$$

Para simplificar a notação, denotaremos sempre por $\tau$ a diferença entre os instantes envolvidos no processo estocástico estacionário, de forma que as funções de correlação sejam denotadas apenas por $C(\tau)$.

Quando $\tau=0$, observamos que recaímos no caso da variância de uma variável aleatória. O cálculo da variância, como feito nas seções anteriores para tratar as flutuação das fotocorrentes detectadas, é uma forma de se caracterizar uma flutuação, porém vimos que a caracterização mais completa de sinais ou processos estocásticos é realizado através do uso de funções de autocorrelação.

\subsubsection{A densidade espectral do processo e sua medida}

O processo estocástico estacionário contínuo $\mathbf{x}(t)$ pode também ser encarado como o resultado de infinitos processos estocásticos de diversas frequências $\omega$. 
Assim, considerando o domínio das frequências, o processo é descrito como a seguinte integral de Fourier:

$$
\mathbf{x}(t)=\int_{-\infty}^{+\infty} \mathbf{X}(\omega) e^{-i \omega t} d \omega
$$

em que $\mathbf{X}(\omega)$ é a sua transformada de Fourier:

$$
\mathbf{X}(\omega)=\frac{1}{2 \pi} \int_{-\infty}^{+\infty} \mathbf{x}(t) e^{i \omega t} d t
$$

Para caracterizar a intensidade de flutuação de cada uma dessas infinitas componentes de frequência $\omega$, é preciso definir outra grandeza denominada espectro de potência ou densidade espectral, denotada por $\mathbf{S}(\omega)$.

A forma mais direta e intuitiva de definí-la seria dizer que ela é proporcional à média do módulo ao quadrado da transformada $\mathbf{X}(\omega)$, entretanto tal definição mostrou-se matematicamente inadequada por apresentar uma divergência. Para resolver essa dificuldade, como demonstrado por Wiener [23], é necessário definir o espectro como:

$$
\mathbf{S}(\omega)=\frac{1}{2 \pi} \int_{-\infty}^{+\infty} C(\tau) e^{i \omega \tau} d \tau
$$

No caso em que os processos estocásticos estacionário são sinais de tensão $V(t)$, como os sinais que lidamos no nosso laboratório, a unidade de medida física dessa função $S(\omega)$ é dada por unidade de potência por largura de banda $(\mathrm{BW}): \frac{W}{H z}$.

Um instrumento de medida que fornece diretamente a medida dessa grandeza é o analisador de espectro. O sinal de entrada nesse instrumento é sempre um sinal de tensão $V(t)$ que é acoplado internamente a uma resistência ${ }^{16}$ $R$ (normalmente de $50 \mathrm{Ohms}$ ) para que este seja capaz de medir a composição espectral $p\left(\omega_{i}\right)$ de potência elétrica do sinal de entrada [18].

Em geral, a unidade de medida fornecida por esse instrumento é o $d B m$, que, diferente do decibel, é uma unidade absoluta de potência. Ele é definido por:

$$
\operatorname{Medida}(\mathrm{dBm})=10 \log \left(\frac{p(W)}{1 m W}\right),
$$

em que $p(W)$ é a potência medida em watt.

Dado um sinal de tensão, esse aparelho fornece a medida de todo o espectro de potência do sinal de entrada dentro de uma janela espectral definida pelo usuário. Normalmente, na nossa prática experimental no LMCAL,

\footnotetext{
${ }^{16}$ Para que o analisador meça potência em uma dada componente de frequência, $p\left(\omega_{i}\right)$, é necessário submeter um resistor a esse sinal de tensão $V\left(\omega_{i}\right)$, pois: $p\left(\omega_{i}\right)=\frac{V^{2}\left(\omega_{i}\right)}{R}$.
} 
utilizamos esse instrumento de medida somente para teste e diagnóstico de problemas em nosso sistema eletrônico.

Entretanto, nas nossas medidas de ruído quântico usamos, como já mencionado, um sistema alternativo conhecido por demodulação, que é um processo eletrônico baseado no mesmo princípio de funcionamento de um analisador de espectro, porém que não fornece a medida direta de $S(\omega)$.

O sinal resultante desse processo de demodulação é um sinal bruto de tensão $V(t)$ somente para uma dada frequência escolhida, chamada de frequência de análise. A análise do espectro de ruído desse sinal $V(t)$ é feito após a medição através de softwares e refere-se somente a intensidade de ruído da frequência de análise escolhida pelo usuário no momento da realização da medida.

Sem entrar em maiores detalhes sobre o processo de medida envolvido, somente para ilustrar, segue, na figura 3.16, um exemplo de medida de $S(\omega)$, usando o analisador de espectro, realizado recentemente no nosso laboratório para investigar problemas de ordem eletrônica. A largura de banda usada na medida foi de $30 \mathrm{KHz}$.

Na medida ilustrada na figura, temos um feixe de $1064 \mathrm{~nm}$, modulado nas frequências $12 \mathrm{MHz}$ e $24 \mathrm{MHz}$, incidente em um detector do tipo descrito na seção 3.1. Conforme visto, quando expostos à luz, os detectores geram fotocorrentes que são amplificadas e transformadas em tensão. O sinal de tensão é, então dividido eletronicamente em sinais de baixa e alta frequência, $D C$ e $H F$ respectivamente, em relação à uma frequência de corte definida pelo circuito do detector.

A informação quântica do feixe é observada através do sinal $H F$ enviado ao analizador de espectro que fornece diretamente a medida de $S(\omega)$. Nessa medida, são observados dois picos de potência exatamente nas frequências $12 \mathrm{MHz}$ e $24 \mathrm{MHz}$ onde sabemos que há modulação.

A ideia da modulação presente em um feixe ficará clara na seção 3.6 onde abordaremos sobre a linguagem usada na engenharia referente à transmissão de informação através da modulação de sinais elétricos. Veremos que essa linguagem é a mesma utilizada para tratar da informação quântica contida em um feixe detectado.

\subsection{A portadora e as bandas laterais}

Faremos nessa seção uma pequena pausa na nossa discussão sobre os processos estocásticos para introduzir uma linguagem frequentemente utilizada na engenharia de telecomunicações para tratar a transmissão de informação via modulação de sinais elétricos e que foi importada para o contexto da 


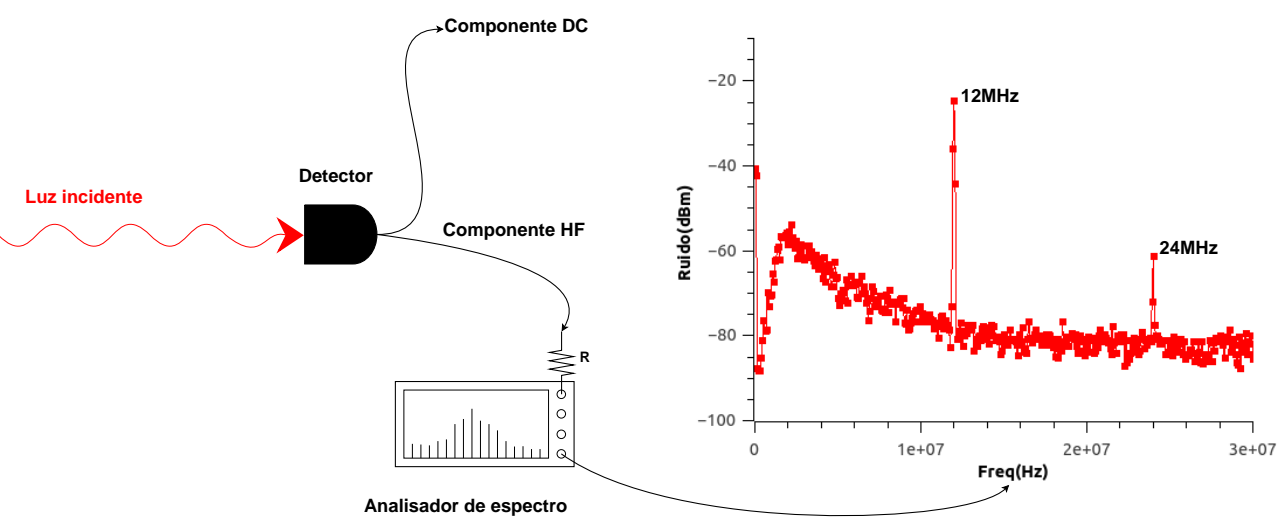

Figura 3.16: Exemplo de uma medida realizada de $S(\omega)$ de um feixe modulado em intensidade nas frequências $12 \mathrm{MHz}$ e $24 \mathrm{MHz}$. O feixe incide no detector, onde é produzida uma fotocorrente. Essa é transformada em um sinal de tensão que é dividido em $D C$ e $H F$. O sinal $H F$ é enviado a um analizador de espectro. À direita da figura, é visto o $S(\omega)$ medido do feixe.

ótica quântica. Além disso, situaremos onde tal linguagem se encaixa nas flutuações da fotocorrente detectada que representa o processo estocástico considerado.

Mais uma vez o objetivo dessa seção não é cobrir de forma matematicamente rigorosa esse assunto e muito menos de forma completa, visto que tal assunto tem desdobramentos complexos em engenharia de telecomunicações. Parte do que falaremos aqui pode ser encontrado em qualquer livro texto de sistemas e sinais, como visto em [16, 30].

Em engenharia, a transmissão de informação contida em um dado sinal elétrico (denominado modulante ou modulador) de frequência muito baixa, relativamente, é feito através da modulação de um outro sinal elétrico com uma frequência muito maior. Tal sinal é denominado de onda portadora e não carrega em si, sem ser modulado, nenhuma informação.

A base de transmissão da informação elétrica é feita através da modulação da portadora usando o sinal modulador. Dois tipos de modulação mais nos interessam por descrever a modulação que ocorre nas fotocorrentes: a modulação da amplitude da portadora, denominada pelos engenheiros de AM(Amplitude Modulation), e a modulação da sua frequência, conhecida como FM(Frequency Modulation). Uma forma de entender como esses tipos de modulação transmitem a informação é considerar que ela está codificada na portadora através da variação intantânea ou de sua amplitude ou da sua frequência.

Assim, podemos dizer que a informação que a portadora carrega em si na 
forma de modulação de amplitude/frequência forma o sinal que é transmitido. Para extrair a informação desse sinal e reconstruí-la na sua forma original é necessário que esse sinal passe por um tratamento eletrônico conhecido por demodulação.

No nosso caso, a informação quântica presente em um feixe pode ser acessada experimentalmente através da análise do espectro de ruído de intensidade da fotocorrente gerada durante a detecção do feixe. Essa informação quântica se manifesta na fotocorrente na forma de ruído que sempre pode ser tratado como resultado de modulações de amplitude e frequência de uma dada portadora [18].

Diferente do que ocorre na engenharia, em que o sinal a ser transmitido é um sinal modulante bem definido e pode ser tratado deterministicamente, a modulação do sinal da portadora da fotocorrente acontece na forma de ruído. Para ilustrar a idéia da portadora e suas modulações em amplitude e frequência, vamos fornecer dois exemplos. Primeiro, iremos analisar casos simples de modulação de amplitude e frequência, em que o sinal modulante é uma função definida. Depois veremos uma simulação de ruído.

\subsubsection{Modulação de amplitude}

Suponhamos, nesse exemplo ilustrativo de modulação, uma onda portadora $x_{p}(t)$ dada por:

$$
x_{p}(t)=A_{p} \cos [\phi(t)]=A_{p} \cos \left(\omega_{p} t\right),
$$

em que $A_{p}$ é a amplitude da portadora, $\phi(t)$ sua fase e $\omega_{p}$ a frequência angular.

Inicialmente, vamos estudar o caso em que a portadora é modulada em amplitude(AM) por um sinal modulante $x_{\text {mod }}(t)$ e, posteriormente, modulada em frequência(FM) por esse mesmo sinal:

$$
x_{\text {mod }}(t)=a_{\text {mod }} \cos \left(\omega_{\text {mod }} t\right)
$$

$a_{m o d}$ é a amplitude da onda modulante e $\omega_{m o d}$ sua frequência angular que, por hipótese, é muito menor que $\omega_{p}$.

Uma forma de modular a amplitude do sinal da equação(3.62) $[16,30]$ ocorre quando somamos a amplitude da portadora com um dado sinal modulador:

$$
x_{A M}(t)=\left[A_{p}+x_{\text {mod }}(t)\right] \cos \left(\omega_{p} t\right),
$$

Esse tipo de modulação é conhecido como $A M$ simples e é muito usado em estações de rádio-difusão [30]. Outra forma de modular a amplitude da portadora, que não será utilizada no nosso contexto, é conhecida como modulação de amplitude com dupla banda lateral e portadora suprimida ou 
$D S B-S C^{17}$. Nesse tipo, a própria amplitude da portadora $A_{p}$ é substituída pelo sinal modulador.

Substituindo-se $x_{\text {mod }}$ da equação (3.64) pela equação (3.63), obtemos o sinal $x_{A M}(t)$ resultante da modulação de amplitude da portadora $x_{p}(t)$ :

$$
x_{A M}(t)=a_{\text {mod }} \cos \left(\omega_{\text {mod }} t\right) \cos \left(\omega_{p} t\right)+A_{p} \cos \left(\omega_{p} t\right)
$$

Nas figuras 3.17 e 3.18 , vemos exemplos numéricos do sinal modulado $x_{A M}(t)$ em que os parâmetros $A_{p}$ e $a_{\text {mod }}$ são iguais a 1 . Em ambas figuras, a frequência angular de modulação, $\omega_{m o d}$, é $2 \pi$ e a frequência angular da portadora é $20 \pi$ na figura 3.17 e $200 \pi$ na figura 3.18 .

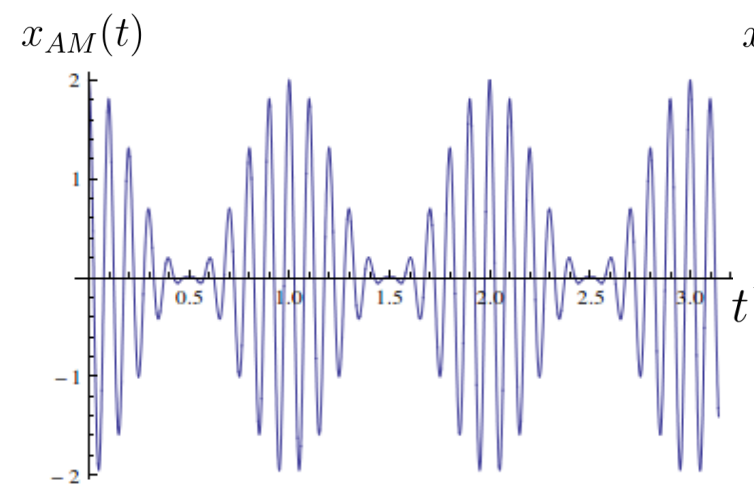

(a)

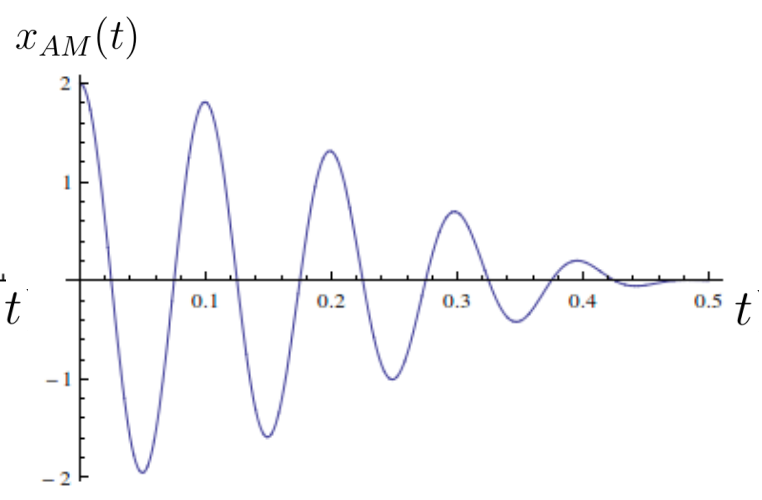

(b)

Figura 3.17: Em (a), é visto o sinal modulado $x_{A M}(t)=\cos (20 \pi t)[1+$ $\cos (2 \pi t)]$ em que a frequência da portadora é igual a 10 unidades arbitrárias $(u . a)$ e a frequência da modulante 1 u.a. Em (b), é ilustrado o mesmo sinal com escala aumentada.

Para eliminar o termo quadrático da equação (3.65) e expressá-la somente como um somatório de cossenos, usamos a seguinte identidade trigonométrica:

$$
\cos \left(\omega_{\text {mod }} t\right) \cos \left(\omega_{p} t\right)=\frac{1}{2}\left[\cos \left(\omega_{\text {mod }}+\omega_{p}\right) t+\cos \left(\omega_{\text {mod }}-\omega_{p}\right) t\right]
$$

Dessa forma, o sinal modulado $x_{A M}(t)$ é reescrito como:

$$
x_{A M}(t)=\frac{a_{m o d}}{2}\left[\cos \left(\omega_{m o d}+\omega_{p}\right) t+\cos \left(\omega_{m o d}-\omega_{p}\right) t\right]+A_{p} \cos \left(\omega_{p} t\right)
$$

De acordo com a equação (3.67), percebemos a presença de três sinais com frequências bem definidas e iguais a $\omega_{p},\left(\omega_{\text {mod }}+\omega_{p}\right)$ e $\left(\omega_{\text {mod }}-\omega_{p}\right)$ que geram

\footnotetext{
${ }^{17}$ Em inglês: Double Sideband-Suppressed Carrier
} 


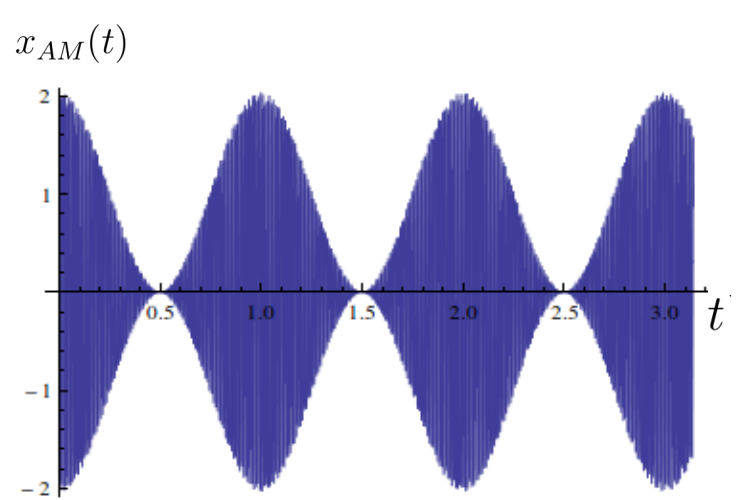

(a)

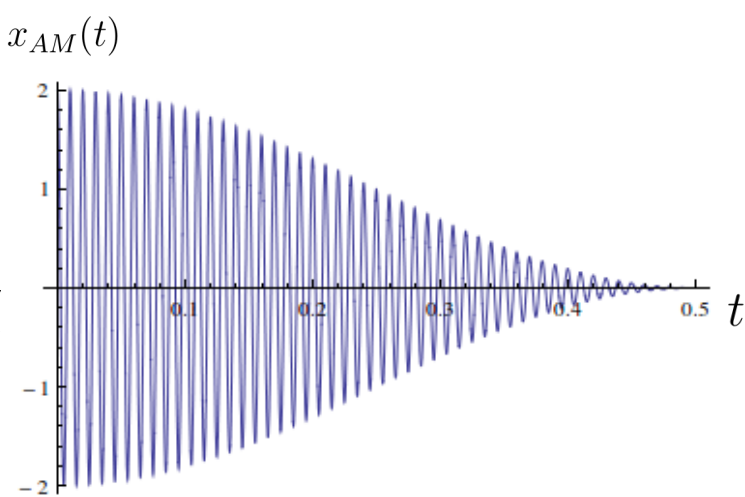

(b)

Figura 3.18: $\operatorname{Em}$ (a), é visto o sinal modulado $x_{A M}(t)=\cos (200 \pi t)[1+$ $\cos (2 \pi t)]$ em que a frequência da portadora é igual a 100 u.a e a frequência da modulante 1 u.a. Em (b), é ilustrado o mesmo sinal com escala aumentada.

a modulação de amplitude. A primeira frequência refere-se à portadora e as outras duas novas frequências, que aparecem naturalmente do processo de modulação, são chamadas de bandas laterais $^{18}$.

Apesar do conceito de bandas laterais ter sido introduzido nesse exemplo particular de sinal modulado, visto na equação (3.63), podemos generalizá-lo e dizer que elas sempre aparecem como resultado de qualquer processo de modulação da portadora seja de amplitude, seja de fase, e que são elas que carregam toda a informação do sinal modulante.

Nos casos de processos estocásticos, como as fotocorrentes, não existe um sinal modulante de frequência bem definida que modula a portadora, mas a presença de infinitos sinais, de amplitude muito menor que a amplitude de portadora, em diversas frequências também muito inferiores a frequência da portadora.

Vejamos qual a contribuição de cada banda lateral produzida no espectro de potência do sinal modulado $x_{A M}(t)$. Nesse caso determinístico, o espectro de potência é simplesmente a transformada de Fourier do próprio sinal modulado em questão, diferente do que consideramos para os processos estocásticos estacionários que levou em conta questões de caráter estatístico, como a correlação.

Usando-se que a transformada de Fourier, denotada por $\mathcal{F}$, da função

\footnotetext{
${ }^{18}$ Essa nomenclatura origina-se no fato de que essas novas frequências situam-se em torno da frequência da portadora $\omega_{p}$.
} 
cosseno é igual a:

$$
\mathcal{F}\left[\cos \left(\omega_{0} t\right)\right]=\pi\left[\delta\left(\omega+\omega_{0}\right)+\delta\left(\omega-\omega_{0}\right)\right]
$$

e as propriedades de linearidade e deslocamento em frequência da transformada, calculamos o espectro de potência $S_{x_{A M}}(\omega)$ do sinal da equação (3.67):

$$
\begin{gathered}
S_{x_{A M}}(\omega)=\frac{\pi a_{\text {mod }}}{2}\left[\delta\left(\omega+\omega_{\text {mod }}+\omega_{p}\right)+\delta\left(\omega-\omega_{\text {mod }}-\omega_{p}\right)+\delta\left(\omega+\omega_{\text {mod }}-\omega_{p}\right)+\right. \\
\left.\quad+\delta\left(\omega-\omega_{\text {mod }}+\omega_{p}\right)\right]+\pi A_{p}\left[\delta\left(\omega+\omega_{p}\right)+\delta\left(\omega-\omega_{p}\right)\right]
\end{gathered}
$$

Assim, a equação (3.69) mostra que o espectro da onda modulada em amplitude apresenta contribuições, com diferentes pesos, referente à portadora e às bandas laterais. Para cada frequência $\omega_{p},\left(\omega_{\text {mod }}+\omega_{p}\right)$ e $\left(\omega_{\text {mod }}-\omega_{p}\right)$, existe um par correspondente de componentes impulsivos, deltas de Dirac, proporcionais a amplitude $A_{p}$, no caso da portadora, e $a_{m o d}$, no caso das bandas laterais.

Uma forma muito usada em engenharia para ilustrar graficamente ${ }^{19} \mathrm{o}$ módulo do espectro de potência de um sinal em função da frequência é representá-lo através de raias de alturas proporcionais aos pesos $\pi A_{p}$ e $\frac{\pi a_{\bmod }}{2}$ localizadas nas suas respectivas frequências, conforme visto na figura 3.19 que representa o módulo do espectro dos sinais das figuras 3.17 e 3.18.

\subsubsection{Modulação de frequência}

Uma outra forma de modular o sinal da portadora é usar o sinal modulante para controlar a sua frequência angular. Ao contrário do caso AM, em que a amplitude da envoltória/envelope da portadora varia em função do sinal modulante, a FM fornece um sinal de amplitude constante independente do modulante, ou seja, o sinal FM tem como característica a propriedade de envelope constante.

Para exemplificar essa nova situação, suponhamos agora que a mesma onda portadora $x_{p}(t)$ tem sua frequência angular variando temporalmente [16] de acordo com a seguinte equação:

$$
\omega(t)=\omega_{p}+G x_{\text {mod }}(t)
$$

$G$ um fator de ganho. Como a derivada temporal da fase, denotada por $\phi(t)$, é igual a frequência angular $\omega(t)$, temos que, consequentemente, a equação

\footnotetext{
${ }^{19}$ Como a área delimitada $($ de $-\infty$ a $+\infty)$ por uma função delta de Dirac é igual a 1 , diz-se que cada componente impulsivo, correspondente à portadora e às bandas laterais, contribui no espectro total com uma área igual a $\pi A_{p}$ e $\frac{\pi a_{\bmod }}{2}$ respectivamente.
} 


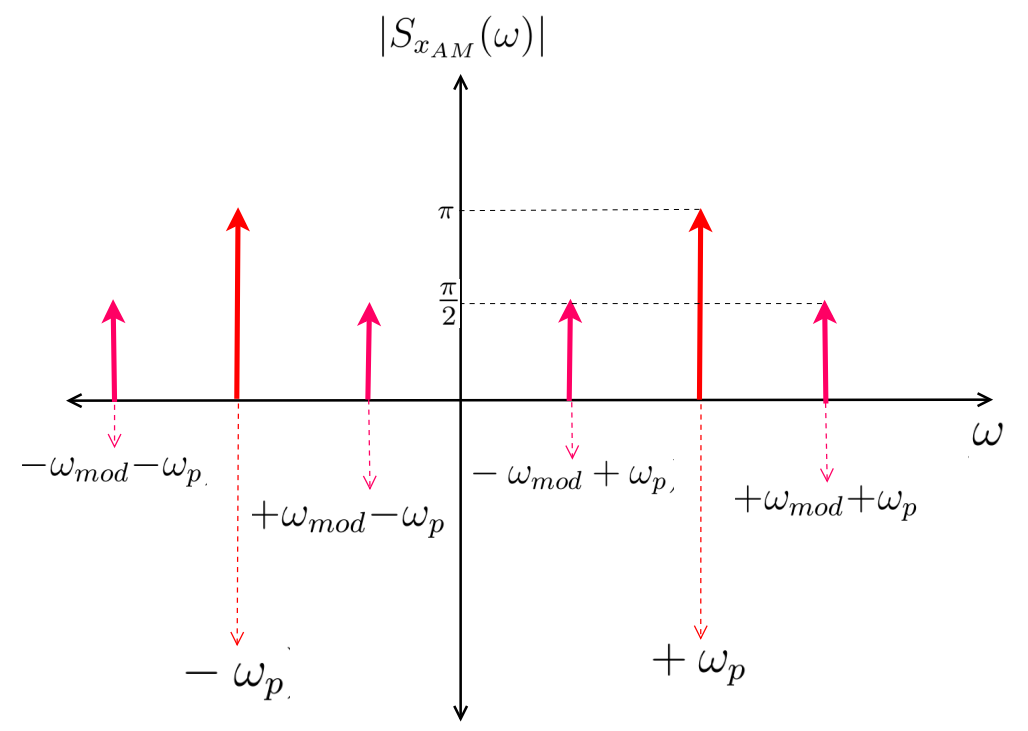

Figura 3.19: Gráfico do módulo do espectro de potência do sinal $x_{A M}(t)$ para $A_{p}=a_{m o d}=1$. Cada função cosseno do sinal (3.67) é responsável por um par impulsivo, representado aqui por setas situadas na respectiva frequência angular a que se refere. Observa-se, além da portadora, em vermelho, o aparecimento de bandas laterais, em rosa, com intensidades menores.

(3.70) provoca uma variação de fase na onda portadora dada por (3.62):

$$
\phi(t)=\omega_{p} t+\int_{0}^{t} G x_{m o d}(t) d t
$$

Assim, a onda modulada em frequência, chamada de $x_{F M}(t)$, é obtida substituindose a equação (6.12) na (3.62):

$$
x_{F M}(t)=A_{p} \cos \left[\omega_{p} t+\int_{0}^{t} G x_{m o d}(t) d t\right]
$$

Para o mesmo sinal modulante visto na (3.63), resulta no sinal:

$$
x_{F M}(t)=A_{p} \cos \left[\omega_{p} t+\frac{a_{\text {mod }} G}{\omega_{\text {mod }}} \operatorname{sen}\left(\omega_{\text {mod }} t\right)\right]
$$

A quantidade $\frac{a_{m o d} G}{\omega_{m o d}}$ é conhecido como índice de modulação FM e denotaremos por $m$. As propriedades dos sinais FM são diferentes dependendo da magnitude do seu índice de modulação. Para valores de $m$ muito menores que $\frac{\pi}{2}$, o sinal FM é dito $F M$ de banda estreita e para valores maiores é conhecido como $F M$ de banda larga [16]. A razão dessa distinção ficará evidente quando tratarmos do espectro de potência do sinal $x_{F M}(t)$. 
Para ilustrar numericamente o sinal FM da equação (3.73) com diferentes índices de modulação, temos as figuras 3.20 e 3.21. Em ambas as figuras, os parâmetros $A_{p}$ e $a_{m o d}$ são iguais a 1 , a frequência angular da portadora é $200 \pi$ e do sinal modulante $2 \pi$. A diferença entre essas figuras está somente no índice $m$ para mostrar como esse parâmetro modifica o aspecto do sinal modulado em frequência.
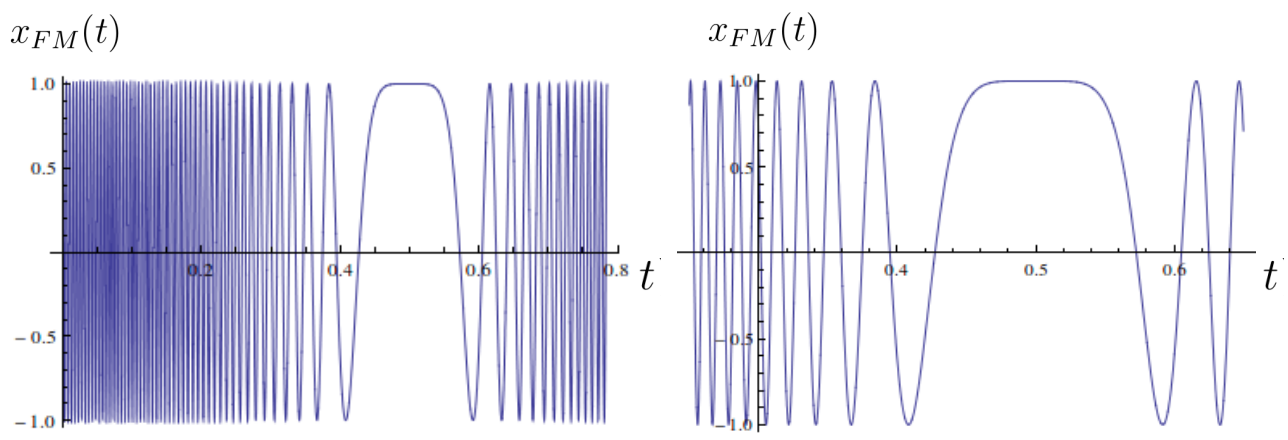

Figura 3.20: $\operatorname{Em}(\mathrm{a})$, é visto o sinal modulado $x_{F M}(t)=\cos [200 \pi t+$ $100 \operatorname{sen}(2 \pi t)]$ em que a frequência da portadora é igual a 100 unidades arbitrárias $(u . a)$ e a frequência da modulante 1 u.a e o parâmetro $m$ é igual a 100. Em (b), é ilustrado o mesmo sinal com escala aumentada.
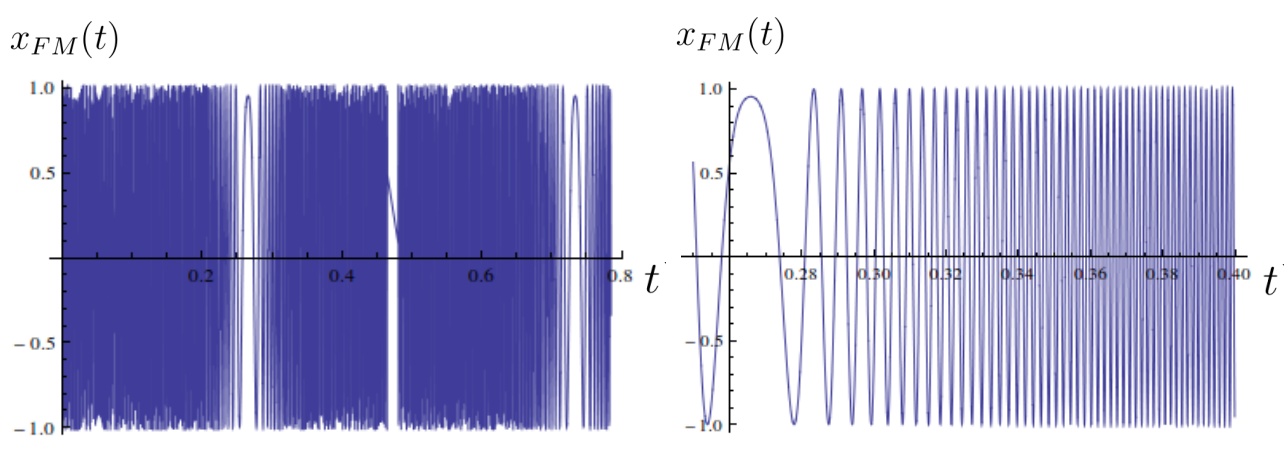

Figura 3.21: $\operatorname{Em}$ (a), é visto o sinal modulado $x_{F M}(t)=\cos [200 \pi t+$ $1000 \operatorname{sen}(2 \pi t)]$ em que a frequência da portadora é igual a 100 u.a e a frequência da modulante 1 u.a e e o parâmetro $m$ é igual a 1000. Em (b), é ilustrado o mesmo sinal com escala aumentada.

Para eliminar o termo $\cos \left[\ldots \operatorname{sen}\left(\omega_{\text {mod }} t\right)\right]$ da equação (3.73), usamos a função geratriz das funções de Bessel expandida em série de Laurent [31]:

$$
e^{\left(\frac{m}{2}\right)\left(w-\frac{1}{w}\right)}=\sum_{n=-\infty}^{+\infty} J_{n}(m) w^{n},
$$


em que os coeficientes $J_{n}(m)$ são definidos como funções de Bessel do $1^{0}$ tipo [31]:

$$
J_{n}(m)=\sum_{s=0}^{+\infty} \frac{(-1)^{s}}{s !(n+s) !}\left(\frac{m}{2}\right)^{n+2 s}
$$

A tabela da figura 3.23 ilustra alguns valores das funções (3.75), para diferentes valores positivos de $n$ e índice de modulação $m$, com duas casas decimais de precisão. Os valores das funções de Bessel para $n$ negativo são facilmente determinados com o uso da seguinte propriedade dessas funções [31]:

$$
J_{-n}(m)=(-1)^{n} J_{n}(m)
$$

Fazendo-se $w=e^{i \omega_{\text {mod }} t}$ na equação (3.74):

$$
e^{i m s e n\left(\omega_{m o d} t\right)}=\sum_{n=-\infty}^{+\infty} J_{n}(m) e^{i n \omega_{m o d} t}
$$

De posse da (3.77), expressamos, finalmente, a equação (3.73) como um somatório de funções cossenos:

$$
x_{F M}(t)=A_{p} \sum_{n=-\infty}^{+\infty} J_{n}(m) \cos \left(\omega_{p} t+n \omega_{m o d} t\right)
$$

Similarmente ao caso da modulação de amplitude, percebemos mais uma vez, através da (3.78), o aparecimento de bandas laterais cujas frequências angular dependem de múltiplos da frequência do sinal modulante. Porém, diferente do caso AM, o número de bandas laterais depende do índice de modulação, $m$, conforme visualizado na tabela 3.23. Na verdade, o número de bandas laterais é o dobro visto na tabela, pois esta só ilustra os valores das funções de Bessel para $n$ positivo. Entretanto, a propriedade (3.76) permite saber os valores de $J_{n}(m)$ para $n$ negativo, que, em módulo, são iguais aos valores para $n$ positivo.

Repetindo-se o mesmo procedimento anterior para calcular o espectro de potência do sinal $x_{F M}(t)$ através da equação (6.36), obtemos:

$$
S_{x_{F M}}(\omega)=\frac{\pi A_{p}}{2} \sum_{n=-\infty}^{+\infty} J_{n}(m)\left[\delta\left(\omega-n \omega_{m o d}-\omega_{p}\right)+\delta\left(\omega+n \omega_{m o d}+\omega_{p}\right)\right]
$$

Novamente, verificamos que, além do número de bandas laterais, a intensidade de cada banda lateral no espectro de potência também depende do índice de modulação $m$. Da tabela 3.23, observamos, por exemplo, o caso 
mais trivial em que a portadora não tem modulação, $m=0$. Nessa situação, existe somente o impulso da portadora e, como não há bandas laterais, ela, obviamente não carrega nenhuma informação, como esperado. Entretanto, de acordo com a mesma tabela, percebemos que, à medida que $m$ cresce, o número de bandas laterais no espectro aumenta, ou seja, a potência do sinal $x_{F M}(t)$ torna-se cada vez mais distruibuída entre diversas componentes de frequência.

Como discutido previamente, para $m \ll \frac{\pi}{2}$, o espectro do sinal FM é caracterizado pelo aparecimento de poucas bandas laterais e, por isso, é dito FM de banda estreita, caso contrário, é conhecido como $F M$ de banda larga.

Para exemplificar situações em que surgem bandas laterais, analisemos dois casos do mesmo sinal FM, dado pela equação (3.73) com $A_{p}=1$, mas com índices de modulação distintos: $m=0,25$ e $m=0,5$. De posse da equação (3.79) e da tabela (3.23), calculamos o espectro de potência do sinal para ambos os índices de modulação e verificamos o aparecimento de bandas laterais de intensidades proporcionais às funções de Bessel. A representação gráfica de ambos os espectros é visualizada na figura 3.22.

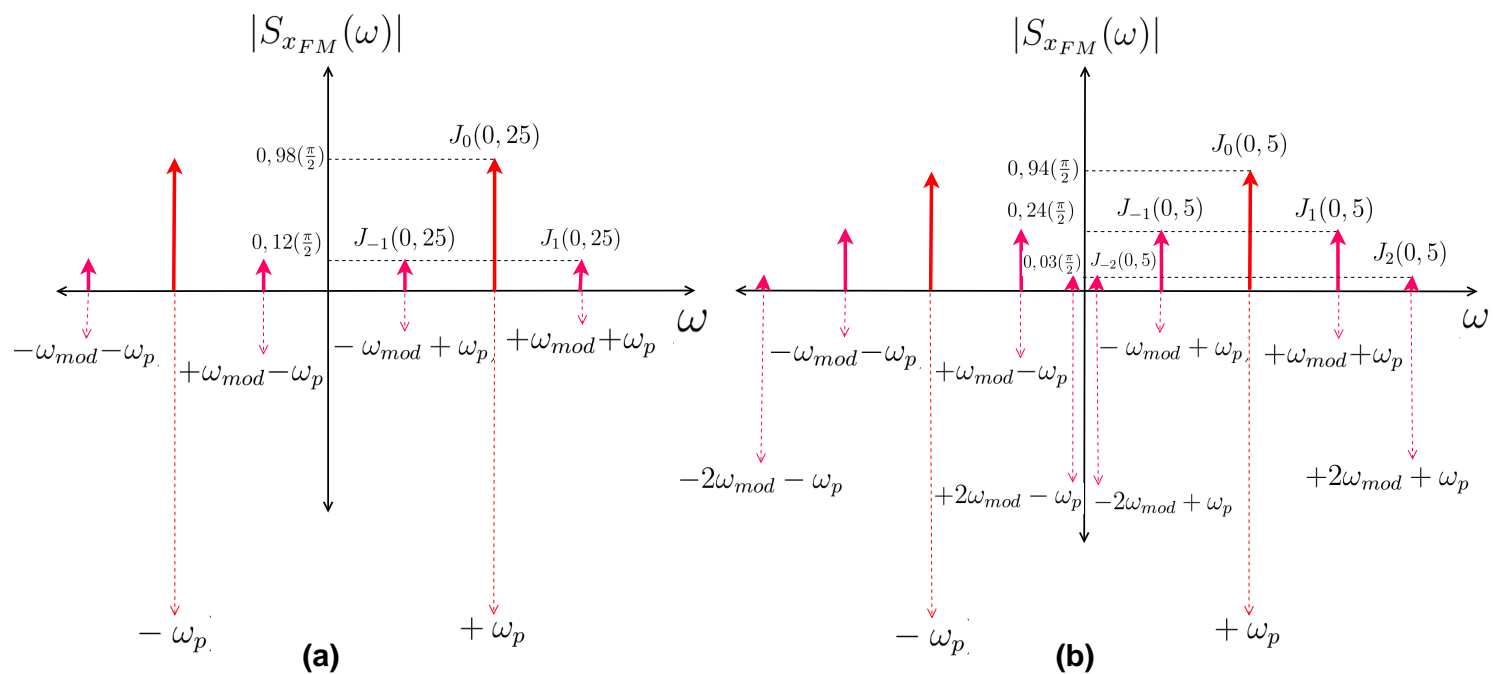

Figura 3.22: Gráficos dos módulos dos espectros de potência do sinal, dado pela equação (3.73) com $A_{p}=1$, com índices de modulação $m=0,25$ e $m=0,5$ respectivamente. Cada par impulsivo é representado por setas situadas nas respectivas frequências angular. Observa-se, além da portadora, em vermelho, o aparecimento de bandas laterais, em rosa, com intensidades menores. O número dessas bandas cresce com o índice de modulação. Em (a), $m=0,25$ e em (b) $m=0,5$. 


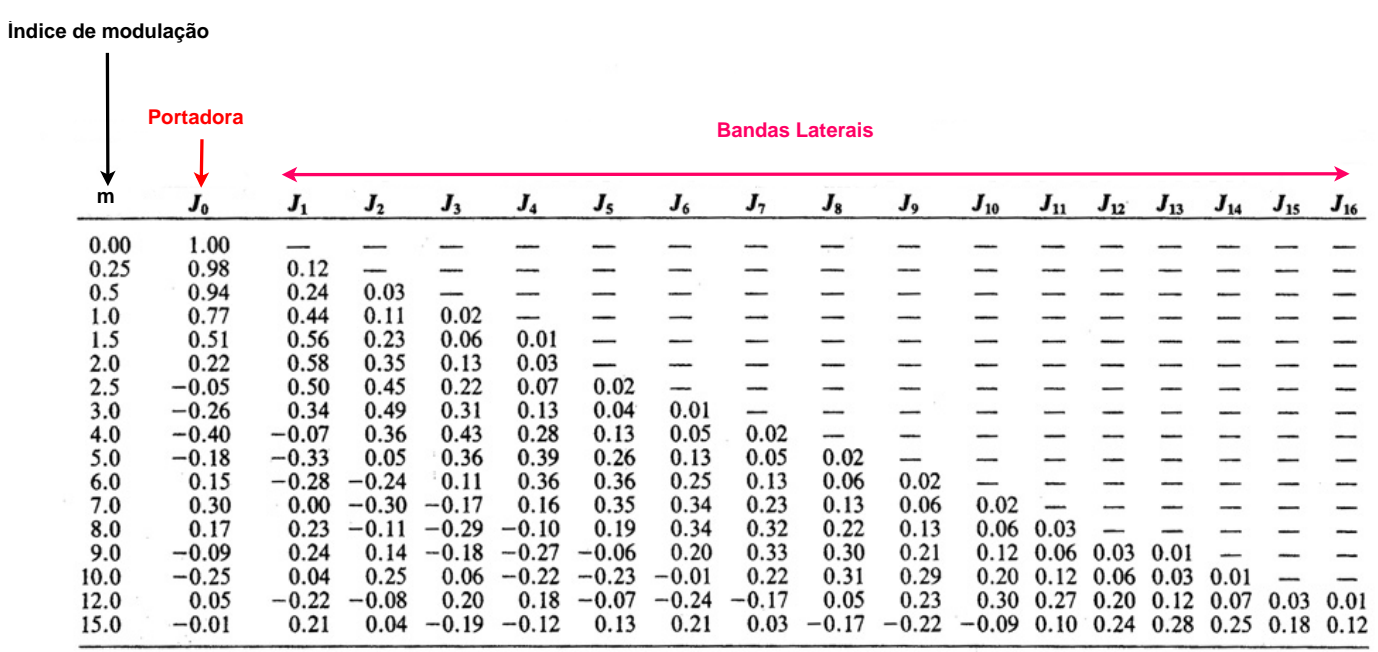

Figura 3.23: Tabela das funções de Bessel do $1^{\underline{0}}$ tipo, para $n$ positivo, com duas casas decimais de precisão. Os traços associados a certos valores de $J_{n}(m)$ indicam valores nulos. De acordo com o índice $m$, observa-se o aparecimento de bandas laterais que dividem a potência do sinal FM com a portadora.

\subsection{Cavidades Óticas}

Antes de abordarmos sobre o método de detecção auto-homodina, cujo o oscilador local é incluso no próprio feixe [8], falaremos primeiro sobre as cavidades óticas. Elas são estruturas óticas muito utilizadas no nosso experimento, pois exercem diversas funções na manipulação dos campos, como veremos ao longo do texto.

Uma cavidade ótica pode ser vista como uma região espacial onde se confinam as ondas eletromagnéticas, utilizando-se, para isso, espelhos devidamente posicionados. Elas são aplicações diretas de problemas de valores de contorno das soluções das equações de Maxwell e podem ter diferentes geometrias. A Figura 3.24 ilustra, esquematicamente, um exemplo de cavidade linear sendo incidida por um campo $\mathbf{E}_{i}$. Os parâmetros que a definem são: a distância entre os espelhos $\frac{L}{2}$ e os seus respectivos coeficientes de reflexão/transmissão de intensidade $\left(R_{1}\right.$ e $\left.R_{2}\right)$.

Por exemplo, consideremos uma dada onda monocromática, polarizada linearmente, incidindo normalmente em um espelho. Para satisfazer as condições de contorno, é preciso existir uma onda refletida pelo espelho e outra transmitida. Como os campos elétricos e magnéticos devem ser contínuos na interface ar/espelho, as amplitudes das ondas refletida e transmitida através do espelho são completamente determinadas pelos índices de refração dos 


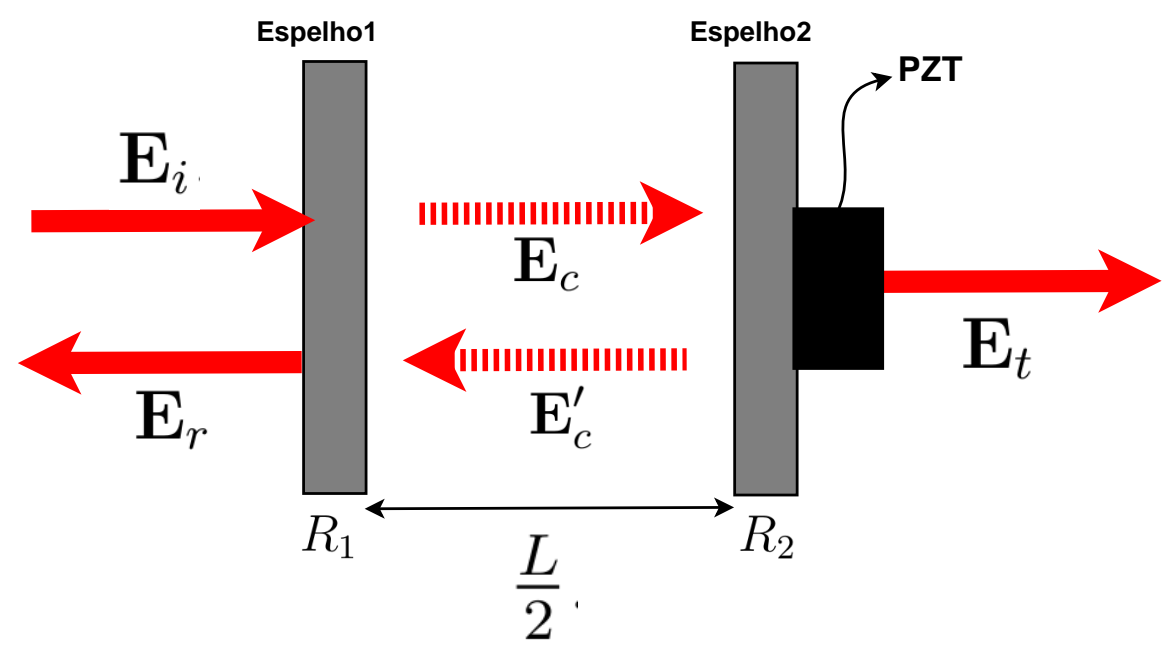

Figura 3.24: Exemplo de uma cavidade ótica linear. Os espelhos 1 e 2 estão separados por uma distância $\frac{L}{2}$ que define o tamanho da cavidade. O espelho 2 está acoplado a um PZT que varia o comprimento da cavidade a fim de alterar as condições de ressonância dentro dela. Os campos $\mathbf{E}_{i}, \mathbf{E}_{r}$ e $\mathbf{E}_{t}$ são os campos incidente, refletido e transmitido pela cavidade. Os campos $\mathbf{E}_{c}$ e $\mathbf{E}_{c}^{\prime}$ são os campos intracavidade.

meios.

As razões entre as amplitudes dos campos incidente e refletido e incidente e transmitido são mais comumente conhecidos como os Coeficientes de Fresnel e dependem do ângulo de incidência da onda incidente na interface (no caso, o espelho).

Uma vez que não se mede os campos elétricos propriamente ditos, mas o fluxo médio de energia (em termos clássicos) por unidade de área, dado pelo vetor de Poynting, ou a intensidade da onda, define-se os coeficientes de transmissão e reflexão de intensidade, $T$ e $R$, como sendo o quadrado dos coeficientes de transmissão $\left(t^{2}\right)$ e reflexão de amplitude $\left(r^{2}\right)$, cuja soma é $T+R=1$. Essa equação diz respeito à conservação de energia na interface (ar/espelho): a intensidade da onda incidente é igual a intensidade da onda transmitida mais a intensidade da refletida pelo espelho.

Como veremos mais adiante, a reflexão $R$ e a transmissão $T$ de intensidade pela cavidade dependem de $R_{1}, R_{2}$ e da distância entre os espelhos. A transmissão de uma dada onda, de uma certa frequência, pela cavidade será máxima quando o comprimento $L$ da cavidade for um múltiplo inteiro da metade do comprimento de onda do campo incidente.

Visto que a cavidade ótica tem um comprimento $\frac{L}{2}$ muitas ordens de grandeza maior que o comprimento de onda do campo incidente, a condição 
de máxima transmissão, ou de ressonância no interior da cavidade, pode ser alcançada, na prática, somente através de um dispositivo denominado PZT que é acoplado a um dos espelhos da cavidade.

O PZT utiliza o fenômeno da piezoeletricidade de um cristal. Esse fenômeno diz respeito à deformação mecânica do cristal ao aplicar-se uma diferença de potencial elétrico sobre ele. Essa deformação é da ordem de grandeza do comprimento de onda da luz e, variando-se o potencial aplicado ao PZT, é possível variar o comprimento da cavidade a ponto de alterar as condições de ressonância dentro dela.

Calculemos agora os campos refletidos e transmitidos pela cavidade. Para isso, vamos supor uma onda do tipo plana $\mathbf{E}_{i}$, monocromática (de frequência igual a $\omega$ ) e polarizada linearmente (com vetor de polarização $\mathbf{E}_{0}$ ), para facilitar, incidindo, com ângulo de incidência $\theta=0$, em uma cavidade formada por dois espelhos dispostos geometricamente de acordo com a Figura 3.24.

Esse problema pode ser resolvido de várias formas, uma delas é considerar as múltiplas reflexões dos feixes dentro da cavidade. Dessa forma, usando-se a notação complexa, podemos escrever a onda incidente como $\mathbf{E}_{i}=\mathbf{E}_{0} e^{i \omega t} \mathrm{e}$ as ondas refletidas, denotadas por $\mathbf{E}_{1 r}, \mathbf{E}_{2 r}, \mathbf{E}_{3 r}, \ldots$ como:

$$
\left\{\begin{array}{l}
\mathbf{E}_{1 r}=r_{1} \mathbf{E}_{0} e^{i \omega t} \\
\mathbf{E}_{2 r}=t_{1}^{2} r_{2} \mathbf{E}_{0} e^{i(\omega t+k L)} \\
\mathbf{E}_{3 r}=t_{1}^{2} r_{1} r_{2}^{2} \mathbf{E}_{0} e^{i(\omega t+2 k L)} \\
\cdots
\end{array}\right.
$$

Assim, podemos encontrar uma relação de recorrência para a n-ésima onda refletida através do espelho 1:

$$
\mathbf{E}_{n r}=t_{1}^{2} r_{1}^{(n-2)} r_{2}^{(n-1)} \mathbf{E}_{0} e^{i[\omega t+k L(n-1)]},
$$

em que $k L$ é a fase adquirida pelas ondas, em relação à $\mathbf{E}_{i}$, devido à diferença de caminho ótico percorrido ao dar uma volta completa na cavidade de tamanho $\frac{L}{2}$. Esses múltiplos feixes refletidos pela cavidade se interferem entre si produzindo $\mathbf{E}_{r}$, que pode ser escrito como o seguinte somatório:

$$
\mathbf{E}_{r}=\mathbf{E}_{0} e^{i \omega t}\left[r_{1}+t_{1}^{2} r_{2} e^{i k L}\left[1+\left(r_{1} r_{2} e^{i k L}\right)+\ldots+\left(r_{1} r_{2} e^{i k L}\right)^{n-2}\right]+\ldots\right],
$$

No limite quando $n \rightarrow \infty$, como $r_{1} r_{2} e^{i k L}<1$, a série converge para $\frac{1}{1-r_{1} r_{2} e^{i k L}}$. Assim,

$$
\mathbf{E}_{r}=\mathbf{E}_{0} e^{i \omega t}\left[r_{1}+\frac{t_{1}^{2} r_{2} e^{i k L}}{1-r_{1} r_{2} e^{i k L}}\right]
$$


Como $t_{1}^{2}+r_{1}^{2}=1$, expressa-se $\mathbf{E}_{r}$ com função somente dos coeficientes de reflexão de amplitude:

$$
\mathbf{E}_{r}=\mathbf{E}_{0} e^{i \omega t}\left[\frac{r_{1}-2 r_{1}^{2} r_{2} e^{i k L}+r_{2} e^{i k L}}{1-r_{1} r_{2} e^{i k L}}\right]
$$

O mesmo raciocínio é aplicado no cálculo do campo transmitido $\mathbf{E}_{t}$ pela cavidade, resultando em:

$$
\mathbf{E}_{t}=\mathbf{E}_{0} e^{i \omega t}\left[\frac{t_{1} t_{2} e^{\frac{i k L}{2}}}{1-r_{1} r_{2} e^{i k L}}\right]
$$

A reflexão $(\mathrm{R})$ e a transmissão $(\mathrm{T})$ de intensidade são definidas como as frações da intensidade dos campos que são refletidos e transmitidos pela cavidade:

$$
\begin{aligned}
& R=\frac{\left|\mathbf{E}_{r}\right|^{2}}{\left|\mathbf{E}_{i}\right|^{2}}, \\
& T=\frac{\left|\mathbf{E}_{t}\right|^{2}}{\left|\mathbf{E}_{i}\right|^{2}} .
\end{aligned}
$$

Assim, substituindo-se as equações (3.83) e (3.84) nas respectivas equações acima, obtemos, finalmente, as funções de reflexão e transmissão da cavidade:

$$
\begin{aligned}
& R=\frac{R_{1}+R_{2}-2 \sqrt{R_{1} R_{2}} \cos (k L)}{1+R_{1} R_{2}-2 \sqrt{R_{1} R_{2}} \cos (k L)}, \\
& T=\frac{T_{1} T_{2}}{1+R_{1} R_{2}-2 \sqrt{R_{1} R_{2}} \cos (k L)} .
\end{aligned}
$$

A Figura 3.25 ilustra um exemplo de funções de transmissão e reflexão de uma cavidade com $T_{1}=T_{2}=R_{1}=R_{2}=0,5$. Da equação (3.88), vemos que a transmissão máxima $\left(T_{\max }\right)$ ocorre quando $k L_{\max }=2 \pi m$ ( $m$ inteiro) e é igual a:

$$
T_{\max }=\frac{T_{1} T_{2}}{\left(1-\sqrt{R_{1} R_{2}}\right)^{2}}
$$

A equação (3.89) implica em um importante resultado quando $R_{1}=R_{2}$. Nessa condição, a transmissão total do campo pela cavidade é igual a 1, ou seja, a luz é totalmente transmitida pela cavidade independente do valor de $R_{1}=R_{2}$.

Uma parâmetro da cavidade ótica muito utilizado é o intervalo espectral livre $^{20}$ (FSR) que é a distância (em frequência) entre a ocorrência de duas

\footnotetext{
${ }^{20}$ Em inglês, free spectral range.
} 


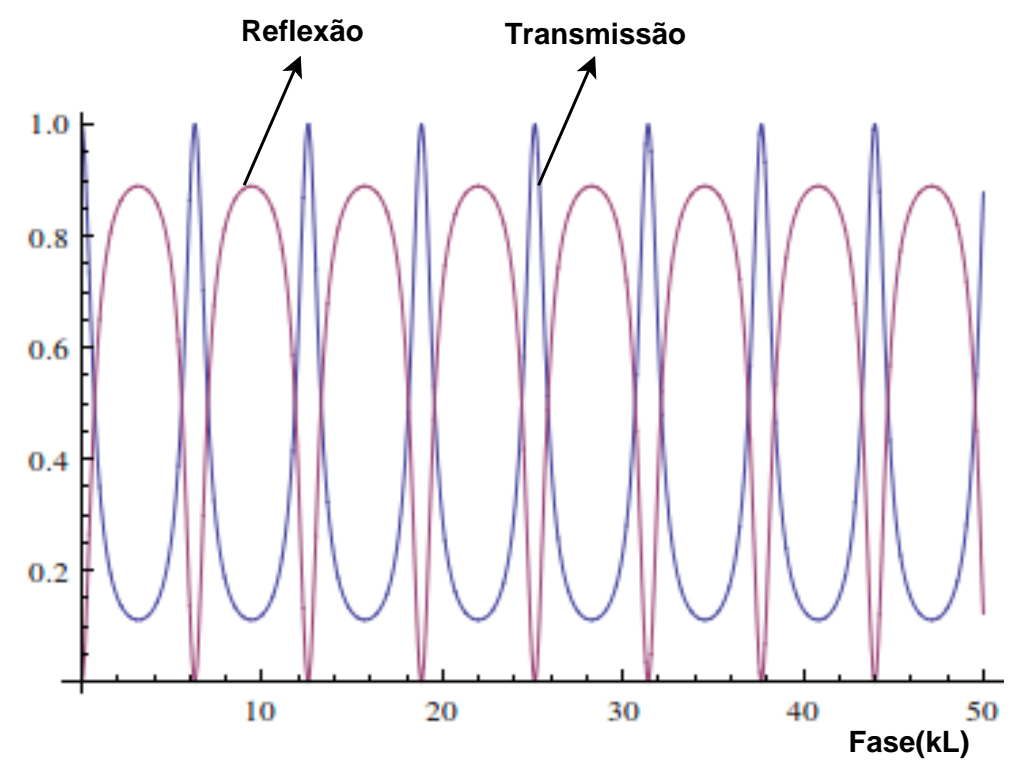

Figura 3.25: Gráficos das funções de transmissão e reflexão para $T_{1}=T_{2}=$ $R_{1}=R_{2}=0,5$.

ressonâncias sucessivas e está relacionado, como vimos, com a condição de máximo da transmissão da cavidade. O FSR é dado por:

$$
F S R=\frac{c}{L}
$$

Outro parâmetro usado é a largura de banda (BW) da cavidade definida como a largura em frequência do pico de ressonância da cavidade. A Figura 3.26 exibe a curva de transmissão da Figura 3.25 e os repectivos parâmetros que a caracterizam.

A razão entre esses dois parâmetros define um outro parâmetro, conhecido por Finesse $F$, que quantifica o quão eficiente é uma cavidade ótica no que concerne o armazenamento de energia da onda eletromagnética incidente:

$$
F=\frac{F S R}{B W}
$$

Esta razão pode ser deduzida teoricamente da seguinte forma. Primeiro, calculemos o BW da cavidade. Para isso, usando relações trigonométricas simples, expressemos a equação (3.88) como:

$$
T=\frac{T_{1} T_{2}}{\left(1-\sqrt{R_{1} R_{2}}\right)^{2}+4 \sqrt{R_{1} R_{2}} \operatorname{sen}^{2}\left(\frac{k L}{2}\right)}
$$




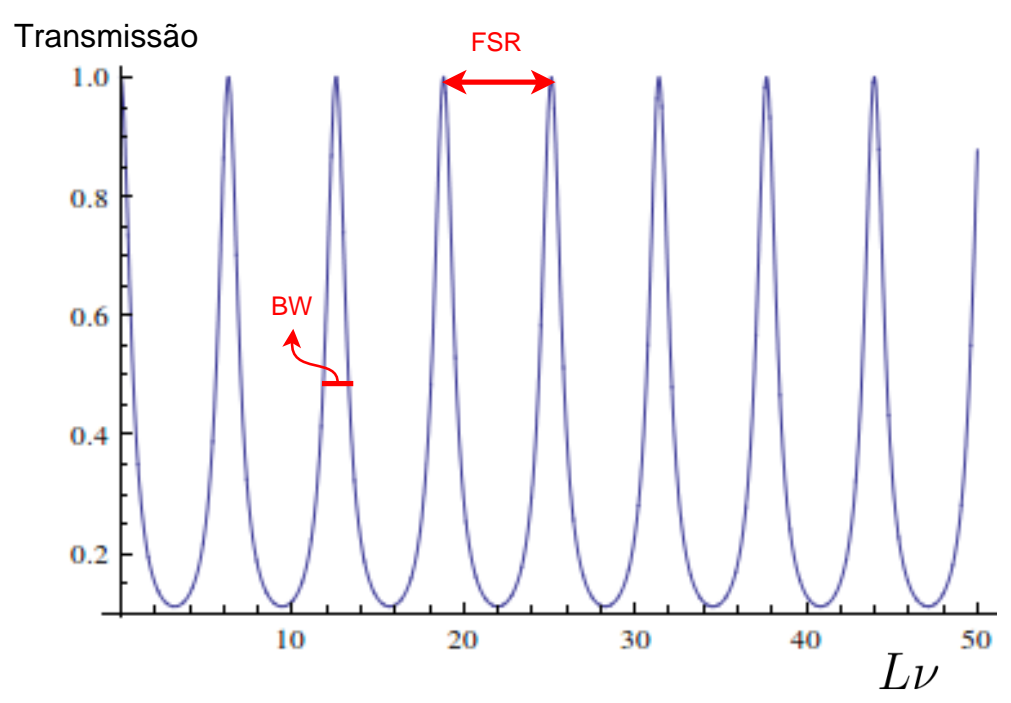

Figura 3.26: Gráfico da função de transmissão para $T_{1}=T_{2}=0,5$, ilustrando o intervalo espectral livre (FSR) e a largura de banda (BW).

Das equações (3.89) e a (3.92), concluimos que a transmissão máxima se reduz a metade quando:

$$
\frac{k L}{2}=\operatorname{sen}^{-1}\left(\frac{\left(1-\sqrt{R_{1} R_{2}}\right)}{2 \sqrt[4]{R_{1} R_{2}}}\right)
$$

A função arcoseno é expandinda em série de Taylor por:

$$
\operatorname{sen}^{-1}(x)=x+\frac{x^{3}}{3 !}+\frac{3^{2} x^{5}}{5 !}+\ldots
$$

Usando-se a primeira aproximação da série (3.94) na equação (3.93) e lembrando que BW é o dobro da equação (3.93), temos que:

$$
B W=\frac{\left(1-\sqrt{R_{1} R_{2}}\right)}{\sqrt[4]{R_{1} R_{2}}}
$$

Substituindo-se (3.95) e $F S R=2 \pi$ na definição (3.91), finalmente, obtemos:

$$
F=\frac{\pi\left(R_{1} R_{2}\right)^{1 / 4}}{1-\sqrt{R_{1} R_{2}}}
$$

Para cavidades com altas transmissões, podemos fazer a aproximação $T_{1} T_{2} \rightarrow$ 0 na equação acima e obter uma relação muito empregada no contexto de cavidades óticas:

$$
F=\frac{2 \pi}{T_{1}+T_{2}}
$$


As cavidades óticas estão presentes no nosso experimento em diversas etapas do processo. No OPO, nosso gerador de estados quânticos emaranhados, a cavidade serve para selecionar as frequências dos feixes gêmeos, realimentandoos. Outra aplicação da cavidade no experimento é a filtragem do feixe do laser, que é feito através do travamento da cavidade na ressonância, de tal forma que o ruído indesejado seja refletido pela cavidade.

Além dessas aplicações, as cavidades óticas, denominadas de análise, também são empregadas no processo de medição das quadraturas do campo, $\hat{X}$ e $\hat{Y}$, através da projeção do ruído de fase em ruído de intensidade [8].

A geometria escolhida para cada uma dessas cavidades depende do comprimento $\frac{L}{2}$. Na cavidade do OPO, por exemplo, o comprimento é pequeno comparada às outras cavidades, assim sua geometria é linear. Já a cavidade de filtro, com 1m de comprimento, e as cavidades de análise possuem o formato de anel. 


\section{Capítulo 4}

\section{Teletransporte Quântico}

De acordo com um teorema muito conhecido, chamado de teorema da nãoclonagem, a informação quântica contida em um dado estado quântico não pode ser copiada, diferente da informação clássica. Dessa forma, qualquer tentativa de manipular a informação quântica requer, primeiro, uma forma eficiente de transmitir essa informação.

O teletransporte quântico surge, então, como uma forma alternativa para transmitir a informação contida nos sistemas quânticos que utiliza tanto via de transmissão clássicas quanto vias de transmissão quântica, aproveitando um tipo de correlação intrinsecamente quântica, o emaranhamento.

Esse capítulo refere-se, inicialmente, sobre o teorema da não-clonagem, o protocolo do teletransporte inicialmente proposto, a fidelidade do processo e, depois, sobre as duas primeiras implementações experimentais em ótica, uma no contexto de variáveis discretas (V.D) e a outra no contexto de variáveis contínuas (V.C).

O intuito deste capítulo é fazer um paralelo entre o que já foi realizado nesse sentido e a nossa proposta de teletransporte bicolor, situando-a dentro desse contexto de informação quântica. Veremos, no próximo capítulo em detalhes, que essa proposta inovadora abrirá portas para a comunicação quântica entre sistemas que operam em diferentes faixas de frequências.

\subsection{O teorema da não-clonagem}

Podemos afirmar que o teorema da não-clonagem foi a primeira grande lei fundamental do que se denomina atualmente por informação quântica, que é a informação codificada nas superposições presentes em todos os sistemas quânticos.

Esse teorema surgiu no início da decáda de 1980 logo após a publicação 
do trabalho de Nick Herbert [32] que propunha o uso de emaranhamento no envio de sinais mais rápidos que a luz, algo que ficou conhecido como "comunicador superluminal", denominado pelo autor de FLASH( "First LaserAmplified Superluminal Hookup").

Obviamente, a transmissão de informação mais rápida que a luz é proibida pela teoria da relatividade especial. Entretanto, o trabalho ${ }^{1}$ serviu para que a comunidade científica se questionasse e investigasse mais a fundo a respeito da transmissão de informação no domínio quântico.

Assim, inspirados pela impossibilidade física de um comunicador superluminal, William Wootters e Wojciech Zurek, em [36], e Dennis Dieks, em [37], provaram, independentemente, o teorema da não-clonagem que estabelece que um estado quântico desconhecido não pode ser copiado, a não ser que ele seja parcialmente conhecido [21].

Como é de esperar-se, não existe análogo desse teorema no âmbito da informação clássica, exatamente porque não existe nenhum princípio físico que impeça a realização de infinitas cópias de uma dada informação clássica [33].

Apesar da sua enorme importância, esse teorema é extremamente simples de ser demonstrado. Fornecemos nesta seção uma prova por absurdo baseado nas referências $[21,33,38]$. Inicialmente, supomos que existe uma "máquina quântica copiadora" capaz de receber um sistema em um estado quântico puro desconhecido, denotado por $|\psi\rangle$, e fazer uma cópia fiel do seu estado.

O sistema físico usado pela "máquina" para copiar está em um outro estado inicial conhecido, denotado por $|\phi\rangle$. A Figura 4.1 é meramente ilustrativa e apresenta uma visão artística da "máquina copiadora" que recebe o estado desconhecido $|\psi\rangle$ e usa o estado $|\phi\rangle$ como base para copiar o estado recebido.

O processo de cópia ou clonagem do estado $|\psi\rangle$ pode ser entendido como uma evolução do sistema global $|\psi\rangle|\phi\rangle$ sujeito a um certo hamiltoniano que não depende do estado $|\phi\rangle$ [21]. Vamos representar esse processo físico de cópia através da atuação de um operador unitário $\hat{U}$ sobre este estado global:

$$
\hat{U}|\psi\rangle|\phi\rangle=|\psi\rangle|\psi\rangle
$$

Se mudarmos a hipótese inicial e supormos que o estado a ser copiado é um

\footnotetext{
${ }^{1} \mathrm{O}$ artigo de Herbert foi aceito apesar dos referees alertarem sobre a impossibilidade de tal comunicador [33]. Inclusive, um desses referees, Asher Peres foi um dos autores do protocolo do teletransporte quântico [34]. Para conhecer mais profundamente a história do teorema, e de como um artigo "equivocado" levou a comunidade científica a um caminho completamente novo e inexplorado da Mecânica Quântica, ver a referência [35] do próprio Asher Peres.
} 


\section{Copiadora de estados quânticos proibida pela MQ!}

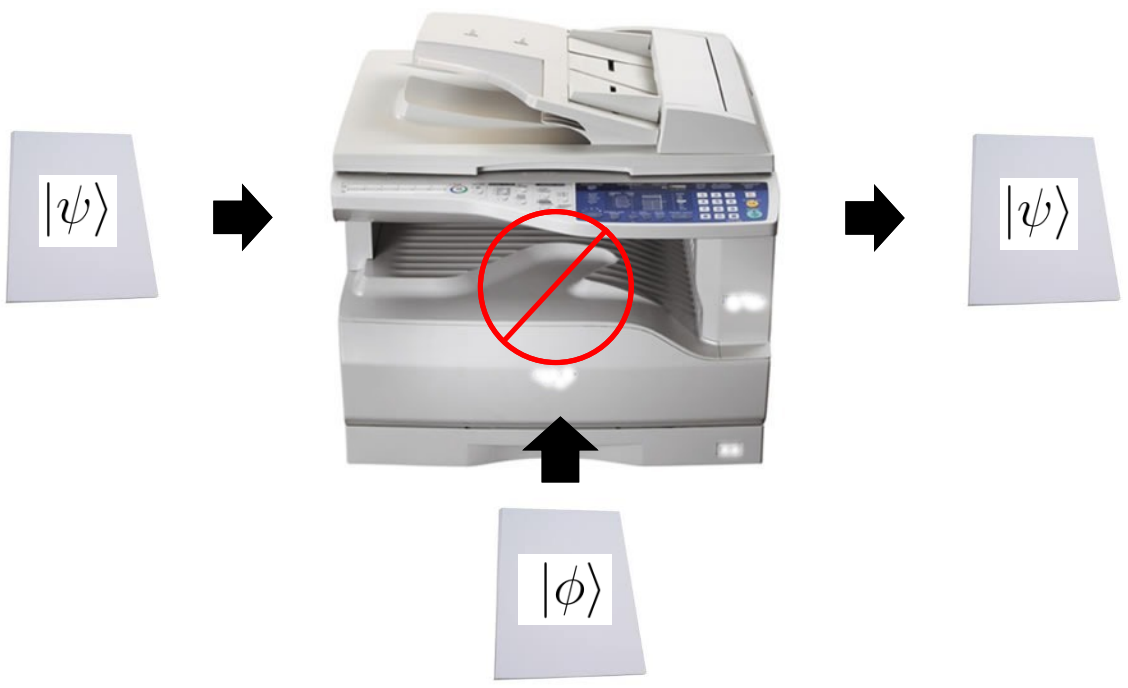

Figura 4.1: Figura artística ilustrativa de uma "copiadora" de estados quânticos. O estado a ser copiado é denotado por $|\psi\rangle$ e o estado inicial usado como base pela copiadora para clonagem é chamado de $|\phi\rangle$.

estado de superposição quântica igual a:

$$
|\psi\rangle=a\left|\psi_{1}\right\rangle+b\left|\psi_{2}\right\rangle
$$

em que os estados $\left|\psi_{1}\right\rangle$ e $\left|\psi_{2}\right\rangle$ são copiáveis, teremos que verificar a equação (4.1) também para este estado. Contudo, a atuação de $\hat{U}$ sobre este estado fornece:

$$
\hat{U}\left(\left(a\left|\psi_{1}\right\rangle+b\left|\psi_{2}\right\rangle\right)|\phi\rangle\right)=a\left|\psi_{1}\right\rangle\left|\psi_{1}\right\rangle+b\left|\psi_{2}\right\rangle\left|\psi_{2}\right\rangle \neq|\psi\rangle|\psi\rangle
$$

Um outra forma alternativa de provar o teorema [38], que trás mais informações sobre a problemática da não-clonagem quântica, é considerar, separadamente, as cópias dos estados $\left|\psi_{1}\right\rangle$ e $\left|\psi_{2}\right\rangle$ :

$$
\begin{aligned}
& \hat{U}\left|\psi_{1}\right\rangle|\phi\rangle=\left|\psi_{1}\right\rangle\left|\psi_{1}\right\rangle, \\
& \hat{U}\left|\psi_{2}\right\rangle|\phi\rangle=\left|\psi_{2}\right\rangle\left|\psi_{2}\right\rangle
\end{aligned}
$$

e calcular o produto interno entre estas equações, lembrando que o operador $\hat{U}$ é unitário:

$$
\left\langle\psi_{1} \mid \psi_{2}\right\rangle=\left(\left\langle\psi_{1} \mid \psi_{2}\right\rangle\right)^{2}
$$


A equação (4.6) obtida apresenta somente duas soluções: $\left\langle\psi_{1} \mid \psi_{2}\right\rangle=1 \mathrm{ou}$ $\left\langle\psi_{1} \mid \psi_{2}\right\rangle=0$, que implica nas duas condições para os estados: ou $\left|\psi_{1}\right\rangle=\left|\psi_{2}\right\rangle$ ou eles são estados ortogonais.

Resumidamente, a máquina copiadora quântica não pode copiar quaisquer estados quânticos, porque existe uma limitação muito clara entre o estado a ser copiado e o estado inicial usado pela "máquina" para copiar, que precisa ser ortogonal ao estado recebido. Logo, uma máquina dessa natureza só não entra em contradição com os axiomas da teoria quântica se obedece essa exigência e, nesse sentido, não é uma copiadora "universal" de estados quânticos.

Por ser algo tão diferente do que observamos classicamente, o teorema da não-clonagem parece, em um primeiro momento, irreconciliável com a física clássica, porém, se analizarmos cuidadosamente a questão, perceberemos que o teorema não proíbe a cópia de estados quânticos, somente impõe uma forte restrição entre os estados quânticos para a realização da cópia.

Como argumenta a referência [38], se pensarmos que a Física clássica nada mais é que um caso especial da Mecânica Quântica, veremos que o fato da informação clássica sempre poder ser copiada pode ser vista como uma particularidade do ponto de vista do teorema da não-clonagem, ou seja, que os diferentes estados clássicos podem ser encarados como estados quânticos ortogonais [38].

É preciso fazer uma ressalva importante a respeito do teorema da nãoclonagem. Ele diz respeito somente a estados quânticos puros, representados por vetores no espaço de Hilbert, porém existe uma generalização desse teorema para estados mais gerais como misturas estatísticas, como apontado em [35], chamado de teorema do não- "broadcasting"2.

Visto a impossibilidade da clonagem quântica e a necessidade emergente de entender como processar a informação de sistemas desse tipo, surge naturalmente uma segunda questão: é possível mover ou reconstruir o estado quântico de um lugar para outro? É nesse contexto que aparece, uma década mais tarde, a proposta do teletransporte de estados quânticos que será abordado na próxima seção.

\subsection{O protocolo original proposto}

A ideia inicial do teletransporte quântico surgiu com a publicação em 1993 do artigo [34] de Charles Bennett, Gilles Brassard, Claude Crépeau, Richard Jozsa, Asher Peres e William Wootters intitulado "Teletransportando um

\footnotetext{
${ }^{2}$ Sem tradução para o português, já que a palavra "broadcasting" é muito usada na nossa língua.
} 
estado quântico desconhecido através de canais clássicos e do tipo EinsteinPodolsky-Rosen". Como dito antes, esse artigo aparece depois dos artigos que culminaram no teorema da não-clonagem como uma consequência natural do processo de investigação da informação codificada em sistemas quânticos.

O termo "teletransporte" usado por eles tem grande apelo no público leigo devido sua inspiração na ficção científica, que diz respeito à possibilidade de fazer desaparecer um objeto em um determinado lugar e fazer aparecer uma réplica idêntica do mesmo em outro lugar.

Porém, diferentemente, no caso do teletransporte quântico, é o estado quântico de uma partícula que é destruído em um lugar e reconstruído em outro através da utilização de dois tipos de canais físicos: clássico e quântico.

Por canal clássico entende-se tudo o que se usa normalmente para transmitir a informação clássica tais como: cabos elétricos, fibras óticas, moduladores eletro-óticos (tratados no capítulo 6). Já por canal quântico, entende-se qualquer sistema emaranhado que possa ser empregado na implementação do protocolo.

O que torna essa proposta tão interessante é justamente a possibilidade do uso de correlações intrinsecamente quânticas na questão da transferência de informação, além de dividir a informação contida em um dado estado quântico em duas partes: uma clássica e outra quântica. O esquema teórico do teletransporte proposto nesse artigo é brevemente discutido a seguir.

Para a realização do protocolo de teletransporte descrito em [34], é necessária a presença de um emissor da informação quântica, denominado usualmente de Alice, de um receptor da informação, denominado Bob, e de canais de comunicação através dos quais a informação, tanto clássica quanto quântica, possa fluir livremente.

Alice deseja, por exemplo, enviar a informação contida no estado $\left|\phi_{1}\right\rangle$ de uma partícula, rotulada por 1, para Bob, que recebe a informação via esses dois tipos de canais de transmissão e reconstrói o estado destruído por Alice $\left(\left|\phi_{1}\right\rangle\right)$.

O estado quântico $\left|\phi_{1}\right\rangle$ desconhecido a ser teletransportado pela emissora Alice ao receptor Bob é escrito como:

$$
\left|\phi_{1}\right\rangle=a\left|\uparrow_{1}\right\rangle+b\left|\downarrow_{1}\right\rangle
$$

sendo $a$ e $b$ coeficientes arbitrários totalmente desconhecidos por Alice. O sistema considerado é um sistema de dois níveis $|\uparrow\rangle$ e $|\downarrow\rangle$ que podem ser os estados de polarização de um fóton ou os estados de um átomo de dois níveis. No contexto da informação quântica, esses estados e suas superposições representam os bits quânticos de informação, conhecidos na literatura como qubits. 
Alice e Bob compartilham um canal quântico que pode ser um par de partículas ou fótons emaranhados, rotulados por 2 e 3 . A partícula rotulada por 2 é dada a Alice e a partícula rotulada por 3 é dada a Bob. O estado quântico desse canal $\left|\Psi_{23}\right\rangle$ é descrito matematicamente pelo produto direto dos estados das partículas 2 e 3 :

$$
\left|\Psi_{23}\right\rangle=\frac{1}{\sqrt{2}}\left(\left|\uparrow_{2}\right\rangle\left|\downarrow_{3}\right\rangle-\left|\downarrow_{2}\right\rangle\left|\uparrow_{3}\right\rangle\right),
$$

e o estado quântico do sistema completo constituído pelas três partículas 1 , 2 e $3\left(\left|\Psi_{123}\right\rangle\right)$ é descrito pelo produto direto dos estados $\left|\phi_{1}\right\rangle$ e $\left|\Psi_{23}\right\rangle$ :

$$
\left|\Psi_{123}\right\rangle=\left|\phi_{1}\right\rangle\left|\Psi_{23}\right\rangle
$$

Logo, calculando-se o produto direto de (4.7) com (4.8), é obtida a expressão para o estado do sistema completo:

$$
\left|\Psi_{123}\right\rangle=\frac{a}{\sqrt{2}}\left(\left|\uparrow_{1}\right\rangle\left|\uparrow_{2}\right\rangle\left|\downarrow_{3}\right\rangle-\left|\uparrow_{1}\right\rangle\left|\downarrow_{2}\right\rangle\left|\uparrow_{3}\right\rangle\right)+\frac{b}{\sqrt{2}}\left(\left|\downarrow_{1}\right\rangle\left|\uparrow_{2}\right\rangle\left|\downarrow_{3}\right\rangle-\left|\downarrow_{1}\right\rangle\left|\downarrow_{2}\right\rangle\left|\uparrow_{3}\right\rangle\right)
$$

Os estados $\left|\phi_{1}\right\rangle$ e $\left|\Psi_{23}\right\rangle$, antes da realização da medida por Alice, são descorrelacionados. Ao realizar uma medida sobre o sistema formado pelas partículas 1 e pela partícula 2, Alice acopla o estado quântico da partícula 1 ao estado emaranhado das partículas 2 e 3.

A medida feita por Alice é conhecida como medida de Bell, nome da base na qual ela projeta o estado global do sistema. Dessa forma, é conveniente representar o estado $\left|\Psi_{123}\right\rangle$ na base de Bell composta pelos quatro estados:

$$
\begin{aligned}
\left|\Psi_{12}^{+}\right\rangle & =\frac{1}{\sqrt{2}}\left(\left|\uparrow_{1}\right\rangle\left|\downarrow_{2}\right\rangle+\left|\downarrow_{1}\right\rangle\left|\uparrow_{2}\right\rangle\right) \\
\left|\Psi_{12}^{-}\right\rangle & =\frac{1}{\sqrt{2}}\left(\left|\uparrow_{1}\right\rangle\left|\downarrow_{2}\right\rangle-\left|\downarrow_{1}\right\rangle\left|\uparrow_{2}\right\rangle\right) \\
\left|\Phi_{12}^{+}\right\rangle & =\frac{1}{\sqrt{2}}\left(\left|\uparrow_{1}\right\rangle\left|\uparrow_{2}\right\rangle+\left|\downarrow_{1}\right\rangle\left|\downarrow_{2}\right\rangle\right) \\
\left|\Phi_{12}^{-}\right\rangle & =\frac{1}{\sqrt{2}}\left(\left|\uparrow_{1}\right\rangle\left|\uparrow_{2}\right\rangle-\left|\downarrow_{1}\right\rangle\left|\downarrow_{2}\right\rangle\right)
\end{aligned}
$$

Esses estados de Bell formam uma base completa e ortogonal do espaço de Hilbert de 4 dimensões, de forma que o estado do sistema das três partículas 1,2 e $3\left(\left|\Psi_{123}\right\rangle\right)$ pode ser escrito, nessa base, como: 


$$
\begin{aligned}
\left|\Psi_{123}\right\rangle=\frac{1}{2}[ & -\left|\Psi_{12}^{-}\right\rangle\left(a\left|\uparrow_{3}\right\rangle+b\left|\downarrow_{3}\right\rangle\right)+\left|\Psi_{12}^{+}\right\rangle\left(-a\left|\uparrow_{3}\right\rangle+b\left|\downarrow_{3}\right\rangle\right)+ \\
& \left.+\left|\Phi_{12}^{-}\right\rangle\left(a\left|\downarrow_{3}\right\rangle+b\left|\uparrow_{3}\right\rangle\right)+\left|\Phi_{12}^{+}\right\rangle\left(a\left|\downarrow_{3}\right\rangle-b\left|\uparrow_{3}\right\rangle\right)\right]
\end{aligned}
$$

Ao medir, Alice projeta o estado do sistema completo em um dos estados de Bell e o estado da partícula 3 é instantaneamente projetado em um dos quatro estados possíveis:

$$
\left\{\begin{array}{l}
\text { Estado (1): }\left(a\left|\uparrow_{3}\right\rangle+b\left|\downarrow_{3}\right\rangle\right) \\
\text { Estado (2): }\left(-a\left|\uparrow_{3}\right\rangle+b\left|\downarrow_{3}\right\rangle\right) \\
\text { Estado (3): }\left(a\left|\downarrow_{3}\right\rangle+b\left|\uparrow_{3}\right\rangle\right) \\
\text { Estado (4): }\left(a\left|\downarrow_{3}\right\rangle-b\left|\uparrow_{3}\right\rangle\right)
\end{array}\right.
$$

Cada um desses quatro estados possíveis tem $\frac{1}{4}$ de probabilidade de ocorrer e todos estão relacionados com o estado $\left|\phi_{1}\right\rangle$, que Alice planeja teletransportar para Bob, a menos de uma operação unitária. Essas transformações unitárias correspondem ao operador identidade e os três operadores de Pauli( denotados por $X, Y$ e $Z)$ :

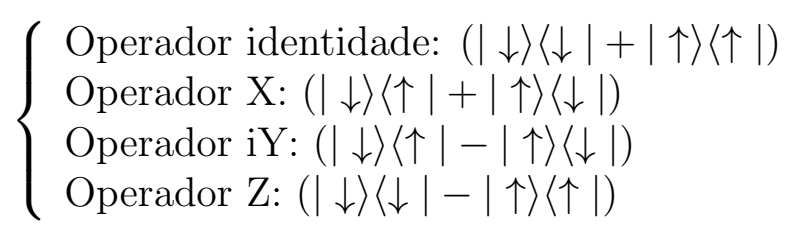

Após a realização da medida, Alice transmite o resultado de suas medidas via canais clássicos para Bob, que aplica um dos operadores unitários sobre o estado da partícula 3 de forma a tornar o estado dessa partícula idêntico ao estado da partícula 1 inicial $\left|\phi_{1}\right\rangle$.

Observando-se os possíveis estados da partícula 3 após a medida de Bell, é percebido que tipo de transformação unitária é necessária que Bob realize para que o processo de teletransporte quântico se complete.

Caso o estado da partícula seja (1), Bob não fará nada, dado que o estado (1) é o próprio estado $\left|\phi_{1}\right\rangle$ que se deseja teletransportar. Caso seja (2), Bob aplicará o operador $X$, o que significa rotacionar esse estado de 180 graus em torno do eixo $x$. Se o estado da partícula 3 for (3), Bob aplicará o operador $i Y$, que rotaciona o estado de 180 graus em torno do eixo $y$, além de mudar a sua fase. Se o estado for (4), Bob aplicará o operador $Z$ que rotaciona o estado de 180 graus em torno do eixo $z$ [33]. Resumidamente, 


$$
\left|\phi_{1}\right\rangle=a\left|\uparrow_{1}\right\rangle+b\left|\downarrow_{1}\right\rangle= \begin{cases}(|\downarrow\rangle\langle\downarrow|+| \uparrow\rangle\langle\uparrow|) & \left(a\left|\uparrow_{3}\right\rangle+b\left|\downarrow_{3}\right\rangle\right) \\ (|\downarrow\rangle\langle\uparrow|+| \uparrow\rangle\langle\downarrow|)\left(-a\left|\uparrow_{3}\right\rangle+b\left|\downarrow_{3}\right\rangle\right) \\ (|\downarrow\rangle\langle\uparrow|-| \uparrow\rangle\langle\downarrow|)\left(a\left|\downarrow_{3}\right\rangle+b\left|\uparrow_{3}\right\rangle\right) \\ (|\downarrow\rangle\langle\downarrow|-| \uparrow\rangle\langle\uparrow|)\left(a\left|\downarrow_{3}\right\rangle-b\left|\uparrow_{3}\right\rangle\right)\end{cases}
$$

Apesar de considerar o teletransporte de um estado puro, o mesmo protocolo também se aplica a estados mais complexos como os estados de mistura estatística, descritos pelo operador densidade $\hat{\rho}$.

A Figura 4.2 ilustra esquematicamente todos os princípios teóricos do protocolo do teletransporte do estado $\left|\phi_{1}\right\rangle$ de Alice para Bob descrito anteriormente.

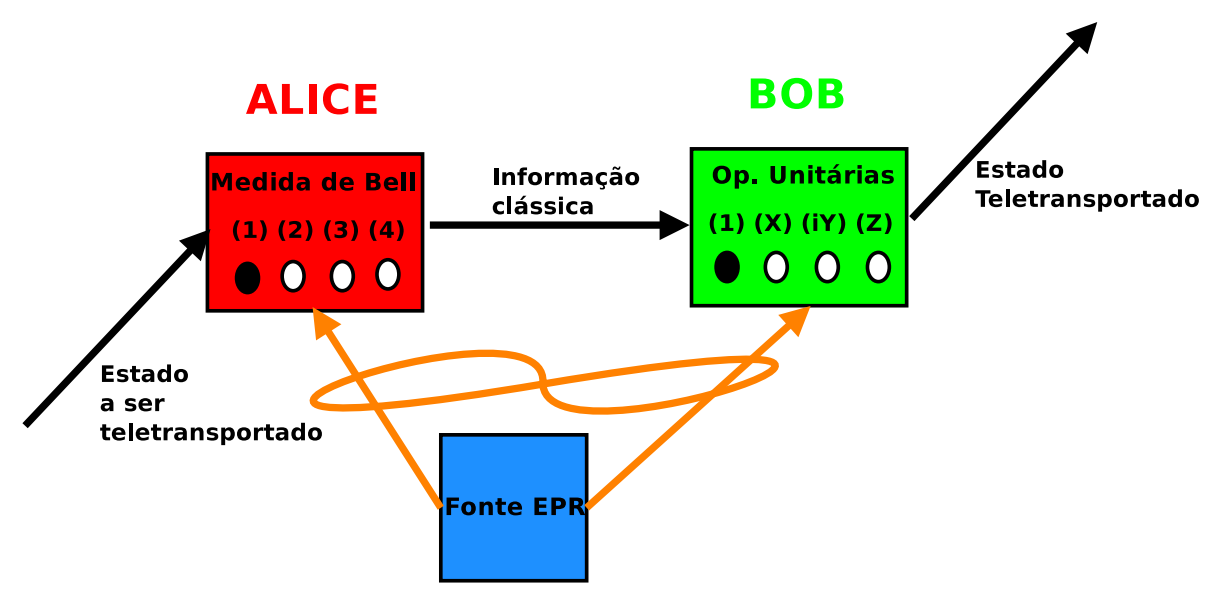

Figura 4.2: Figura adaptada da referência [39] que ilustra o protocolo de teletransporte quântico proposto em [39]. Uma fonte de partículas emaranhadas distribui suas partículas para Alice e Bob. Alice recebe o estado a ser teletransportado e, juntamente com a partícula recebida da fonte, realiza uma medida de Bell. Após a medida, Alice transmite classicamente seus resultados para Bob, que reconstrói o estado com o auxílio da partícula emaranhada recebida da fonte.

É necessário destacar alguns aspectos a respeito deste protocolo. No teletransporte, o estado $\left|\phi_{1}\right\rangle$ a ser transmitido é desconhecido por Alice e Bob durante todo o processo, além disso, o estado da partícula 1, inicialmente $\left|\phi_{1}\right\rangle$, é destruído, dado que Alice realiza uma medida do tipo Bell sobre o sistema composto pelas partículas 1 e 2 , o que garante que o teorema da não-clonagem não seja violado [36, 37].

Outro ponto importante a se mencionar é de novo sobre a essência da transmissão de informação de Alice para Bob que, aparentemente, parece 
violar a relatividade restrita, dado que o estado da partícula 3 é alterado com a medida feita por Alice. Porém, essa contradição é, mais uma vez, enganosa, pois essa alteração ocorre devido à característica não-local do canal quântico presente no processo.

Se analizarmos, com cuidado, a transmissão da informação completa para Bob, percebemos que ele só pode reconstruir o estado teletransportado depois que recebe as informações clássicas de Alice, sujeitas obviamente ao limite de velocidade de transmissão imposto pela relatividade.

Desde o surgimento do protocolo do teletransporte de Bennett et al [34], várias implementações tanto teóricas quanto experimentais foram propostas em variáveis discretas, algumas usando o estado quântico de um átomo de dois níveis; estados internos e vibracionais de íons armadilhados ou ainda usando estados de polarização de um fóton ${ }^{3}$ [39].

Outras implementações também foram feitas adaptando o protocolo de [34] para variáveis contínuas. Exemplos disso são: a primeira proposta para a posição e o momento de uma partícula [40] e as primeiras propostas experimentais para estados coerentes [7] e estados comprimidos [41].

Destaca-se também o desenvolvimento de tecnologias que permitiram ir mais além e realizar o teletransporte de uma parte de um estado emaranhado $[42,43]$ tanto em V.D quanto em V.C, técnica conhecida como " entanglement swapping, e, depois, o teletransporte de um estado emaranhado inteiro [44].

Essas técnicas representaram um grande avanço em V.C, pois possibilitaram que a compressão dos estados teletransportados fosse mantida [44], diferente do teletransporte de um estado comprimido, visto em [41], onde a compressão do estado não era preservada. Recuperar a informação da compressão do estado quântico inicial é essencial se quisermos extender o protocolo a um esquema de teletransporte em cascada [44].

Em um trabalho inédito mais recente [45], verifica-se também a realização do teletransporte em um esquema experimental dito híbrido em que os autores implementaram o protocolo usando o teletransporte em variáveis contínuas de estados quânticos discretos, mais especificamente os estados de polarização de fótons, que representam os qubits. Em suma, os autores realizaram o teletransporte de qubits de uma forma determinística ou "incondicional", sem o caráter probabilístico de sucesso típico do teletransporte em variáveis discretas.

Além disso, muitos esforços tem sido feitos, em variáveis discretas, para implementar o protocolo em distâncias cada vez maiores, como visto nas

\footnotetext{
${ }^{3}$ Sabe-se que a polarização é uma característica clássica do campo,que descreve o modo, não o estado do campo. Nesse texto, refere-se ao estado de polarização de um fóton como um estado em que há a superposição quântica de um fóton propagando com polarização horizontal e um fótons se propagando com polarização vertical.
} 
referências [46, 47, 48], o que possibilitará em um futuro, talvez distante, a realização do teletransporte de qubits para satélites, permitindo a criação de uma rede quântica global.

Para contextualizar melhor o protocolo de [34], serão descritas, nas seções 4.4 e 4.5, duas realizações experimentais: uma em variáveis discretas, o teletransporte de estados de superposição da polarização de um fóton [39], e a outra, em variáveis contínuas, o teletransporte de estados coerentes óticos [7]. Atenção especial é dada a essa implementação porque foi a partir dela que nos baseamos para propor o nosso esquema de teletransporte bicolor.

\subsection{A fidelidade do teletransporte}

Antes de seguir com os primeiros experimentos que implementaram o teletransporte quântico, faremos uma pequena pausa na discussão para falar de uma métrica no espaço de Hilbert que permite verificar a semelhança entre estados quânticos. Não entraremos nas minúcias sobre as normas de distância em informação quântica nesta seção, apenas enunciaremos o principal resultado obtido pelos teóricos para o teletransporte de estados coerentes.

Em qualquer esquema ideal de teletransporte, tanto em variáveis discretas quanto em variáveis contínuas, o estado reconstruído por Bob é exatamente o estado quântico enviado por Alice. Porém, sabemos que sistemas físicos reais estão sujeitos a perdas espúrias e que, principalmente, uma fonte de estados emaranhados nunca é maximamente emaranhada. Logo, o estado reconstruído por Bob não é exatamente o estado recebido.

Para quantificar a distância entre esses estados quânticos e o limite a partir do qual é possível afirmar, teoricamente, que ocorreu o teletransporte, existe uma medida denominada fidelidade quântica (F) $[38,49,50]$. Esta métrica é fundamental para que os físicos experimentais consigam afirmar se o teletransporte ocorreu devido ao canal quântico ou emaranhamento envolvido na implementação.

Para um estado de entrada puro $\left|\psi_{\text {in }}\right\rangle$ e um estado de saída arbitrário $\hat{\rho}_{\text {out }}$, uma mistura estatística, a fidelidade é definida por:

$$
F \equiv\left\langle\psi_{\text {in }}\left|\hat{\rho}_{\text {out }}\right| \psi_{\text {in }}\right\rangle
$$

Esta medida é, obviamente, igual a 1 se o estado quântico final for o estado puro $\hat{\rho}_{\text {out }}=\left|\psi_{\text {in }}\right\rangle\left\langle\psi_{\text {in }}\right|$, que é o caso ideal. Entretanto, esta medida é igual a zero se os estados quânticos, recebido por Alice e reconstruído por Bob, forem ortogonais ou se forem distinguíveis por alguma medida quântica [50].

Outro aspecto importante refere-se a fidelidade do teletransporte de estados coerentes que é justamente o tipo de estado que nos propomos a te- 
letransportar nesse trabalho. Prova-se $[50,51]$ que, nesse caso, a fidelidade média $\left(F_{\text {med }}\right)$ em um teletransporte quântico, necessariamente, é:

$$
F_{\text {med }}>\frac{1}{2}
$$

Assim, (4.17) é o critério que indica se a transmissão de informação de Alice para Bob ocorreu de forma clássica ou quântica com o uso do emaranhamento.

\subsection{Teletransporte em variáveis discretas}

O trabalho de Bouwmeester et al [39] publicado em 1997 intitulado "Teletransporte quântico experimental" é um dos trabalhos mais conhecidos sobre teletransporte de um qubit exatamente por ter sido a primeira realização experimental do teletransporte. O protocolo foi implementado usando fótons produzidos por conversão paramétrica descendente, mesmo processo físico que usamos no laboratório ${ }^{4}$, e medidos em uma base de modos de polarização.

O esquema geral do arranjo experimental de [39] é visto na Figura 4.3. Para a realização do experimento, é necessário produzir o estado emaranhado, assim como medi-los. Na sua produção, é usado um laser de bombeio pulsado na região do utravioleta passando por um cristal não-linear que, através conversão paramétrica descendente, produz o par dos fótons 2 e 3 vistos na figura 4.3. Além desse par de fótons emaranhados, esse mesmo esquema produz, na reflexão, outro par de fótons igualmente emaranhados 1 e 4 . O estado do fóton 1 é o estado teletransportado e o fóton 4 serve como "gatilho" de todo o processo.

Como a grandeza física observada é o estado de polarização do fóton, o fóton 1 passa por um polarizador (denotado por Pol na figura) de forma a preparar seu estado de polarização desconhecido por Alice e Bob. O estado preparado para o fóton 1 é do tipo (4.7) e o estado emaranhado das partículas 2 e 3 é exatamente o descrito por (4.8).

Para acompanhar a notação do artigo [39], o estado do fóton 1 e das partículas emaranhadas 2 e 3 são dados em termos das polarizações horizontal e vertical descritos respectivamente por:

$$
\begin{gathered}
\left|\psi_{1}\right\rangle=\alpha\left|\leftrightarrow_{1}\right\rangle+\beta\left|\uparrow_{1}\right\rangle, \\
\left|\psi_{23}\right\rangle=\frac{1}{\sqrt{2}}\left(\left|\leftrightarrow_{2}\right\rangle\left|\uparrow_{3}\right\rangle-\left|\uparrow_{2}\right\rangle\left|\leftrightarrow_{3}\right\rangle\right),
\end{gathered}
$$

\footnotetext{
${ }^{4}$ porém, em um outro regime de operação onde produz-se feixes intensos.
} 


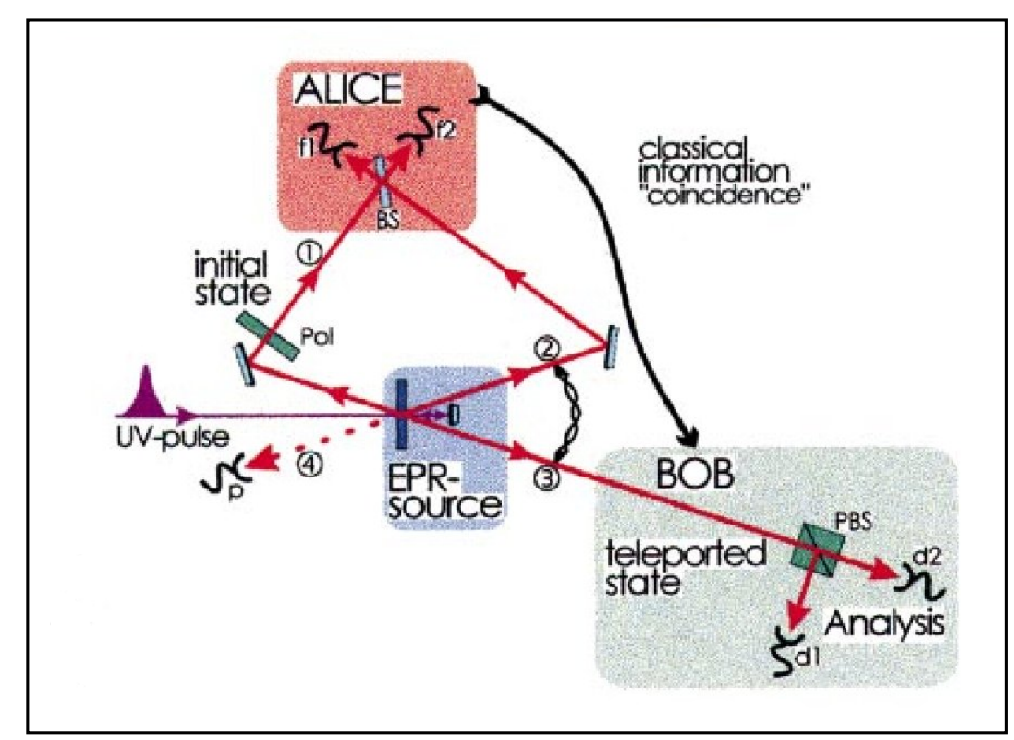

Figura 4.3: Figura da referência [39]. Montagem do primeiro experimento de teletransporte em váriaveis discretas realizado. O protocolo utilizado foi o mesmo descrito em [34].

Ao ser disparado o "gatilho" pelo fóton 4 da Figura 4.3, Alice se prepara para começar a medir. Essa medida, de acordo com o protocolo de Bennett et al em [34], refere-se à projeção do estado do sistema formado pelos fótons 1 e $2\left(\left|\Psi_{12}\right\rangle\right)$ na base de Bell. Em termos experimentais, isso é feito incidindo ambos os fótons separadamente em um BS.

\subsubsection{O papel do BS na medida de Bell}

No caso específico do esquema experimental da Figura 4.3 do artigo [39], a relação entre os operadores aniquilação $\hat{a}$ associados aos estados $\leftrightarrow \mathrm{e} \uparrow$ dos fótons 1 e 2 nos detectores $f 1$ e $f 2$, após o BS, são denotados ${ }^{5}$ respectivamente por:

$$
\left\{\begin{array}{lll}
\hat{a}_{\leftrightarrow}^{f 1}=\frac{1}{\sqrt{2}}\left(\hat{a}_{\leftrightarrow}^{\text {foton } 1}+\hat{a}_{\leftrightarrow}^{\text {foton } 2}\right) & e & \hat{a}_{\leftrightarrow}^{\text {f2 }}=\frac{1}{\sqrt{2}}\left(\hat{a}_{\leftrightarrow}^{\text {foton } 1}-\hat{a}_{\leftrightarrow}^{\text {foton } 2}\right) \\
\hat{a}_{\uparrow}^{f 1}=\frac{1}{\sqrt{2}}\left(\hat{a}_{\uparrow}^{\text {foton } 1}+\hat{a}_{\uparrow}^{\text {foton } 2}\right) & e & \hat{a}_{\uparrow}^{\text {fo }}=\frac{1}{\sqrt{2}}\left(\hat{a}_{\uparrow}^{\text {foton } 1}-\hat{a}_{\uparrow}^{\text {foton } 2}\right)
\end{array}\right.
$$

O estado de entrada no BS em Alice é o estado do sistema formado pelos fótons polarizados 1 e 2. Como qualquer estado do sub-espaço de Hilbert considerado pode ser construído a partir da ação do operador criação $\hat{a}^{\dagger}$ sobre

\footnotetext{
${ }^{5}$ Adaptou-se nesse texto a notação adotada no livro [33] para esses operadores.
} 
o vácuo e os fótons 1 e 2 estão em estados de polarização perpendicular, esse estado $\left|\Psi_{12}\right\rangle$ é representado como:

$$
\left|\Psi_{12}\right\rangle=\left(\hat{a}_{\leftrightarrow}^{\text {foton } 1}\right)^{\dagger}\left(\hat{a}_{\uparrow}^{\text {foton } 2}\right)^{\dagger}|0\rangle|0\rangle
$$

Experimentalmente, após o BS, ocorre a detecção simultânea dos fótons 1 e 2 nos detectores $f 1$ e $f 2$. Do formalismo teórico, essa ação implica em projetar (4.20) sobre o bra $\langle 0|\langle 0|\left(\hat{a}_{\leftrightarrow}^{f 1}\right)\left(\hat{a}_{\uparrow}^{f 2}\right)$ :

$$
\langle 0|\left\langle 0\left|\left(\hat{a}_{\leftrightarrow}^{f 1}\right)\left(\hat{a}_{\uparrow}^{f 2}\right)\right| \Psi_{12}\right\rangle
$$

Das relações obtidas anteriormente para o BS, calcula-se o operador $\left(\hat{a}_{\leftrightarrow}^{f 1} \hat{a}_{\uparrow}^{f 2}\right)$ em termos dos operadores $\hat{a}_{\leftrightarrow}^{\text {foton } 1}, \hat{a}_{\uparrow}^{\text {foton } 1}, \hat{a}_{\leftrightarrow}^{\text {foton } 2}, \hat{a}_{\mathfrak{1}}^{\text {foton } 2}$ :

$$
\left(\hat{a}_{\leftrightarrow}^{f 1} \hat{a}_{\uparrow}^{f 2}\right)=\frac{1}{2}\left(\hat{a}_{\leftrightarrow}^{\text {foton } 1} \hat{a}_{\uparrow}^{\text {foton } 1}-\hat{a}_{\leftrightarrow}^{\text {foton } 1} \hat{a}_{\uparrow}^{\text {foton } 2}+\hat{a}_{\leftrightarrow}^{\text {foton } 2} \hat{a}_{\uparrow}^{\text {foton } 1}-\hat{a}_{\leftrightarrow}^{\text {foton } 2} \hat{a}_{\uparrow}^{\text {foton } 2}\right)
$$

Como só um fóton é teletransportado e também só um fóton é enviado para Alice, pela própria construção do experimento, então as probabilidades de detecção de dois fótons teletransportados e dois fótons vindos da fonte EPR para Alice são nulas [33]:

$$
\langle 0|\left\langle 0\left|\hat{a}_{\leftrightarrow}^{\text {foton } 1} \hat{a}_{\uparrow}^{\text {foton } 1}\right| \Psi_{12}\right\rangle=0 \quad e \quad\langle 0|\left\langle 0\left|\hat{a}_{\leftrightarrow}^{\text {foton } 2} \hat{a}_{\uparrow}^{\text {foton } 2}\right| \Psi_{12}\right\rangle=0
$$

Finalmente, de posse das equações (4.22) e (4.23), é possível calcular (4.21):

$$
\langle 0|\left\langle 0\left|\left(\hat{a}_{\leftrightarrow}^{f 1}\right)\left(\hat{a}_{\uparrow}^{f 2}\right)\right| \Psi_{12}\right\rangle=\frac{1}{2}\left(-\langle 0|\left\langle 0\left|\hat{a}_{\leftrightarrow}^{\text {foton } 1} \hat{a}_{\uparrow}^{\text {foton } 2}\right| \Psi_{12}\right\rangle+\langle 0|\left\langle 0\left|\hat{a}_{\leftrightarrow}^{\text {foton } 2} \hat{a}_{\uparrow}^{\text {foton } 1}\right| \Psi_{12}\right\rangle\right)
$$

Após a atuação dos operadores aniquilação em (4.24), (4.21) resulta em:

$$
\langle 0|\left\langle 0\left|\left(\hat{a}_{\leftrightarrow}^{f 1}\right)\left(\hat{a}_{\uparrow}^{f 2}\right)\right| \Psi_{12}\right\rangle=\frac{1}{2}\left(-\left\langle\leftrightarrow_{1}\right|\left\langle\uparrow_{2} \mid \Psi_{12}\right\rangle+\left\langle\leftrightarrow_{2}\right|\left\langle\uparrow_{1} \mid \Psi_{12}\right\rangle\right)
$$
é:

Lembrando-se que o vetor $\Psi_{12}^{(-)}$da base de Bell, na notação do artigo [39],

$$
\left|\Psi_{12}^{-}\right\rangle=\frac{1}{\sqrt{2}}\left(\left|\leftrightarrow_{1}\right\rangle\left|\uparrow_{2}\right\rangle-\left|\uparrow_{1}\right\rangle\left|\leftrightarrow_{2}\right\rangle\right)
$$

É possível reescrever (4.25) na base de Bell como: 


$$
\begin{aligned}
\langle 0|\left\langle 0\left|\left(\hat{a}_{\leftrightarrow}^{f 1}\right)\left(\hat{a}_{\uparrow}^{f 2}\right)\right| \Psi_{12}\right\rangle & =\frac{1}{\sqrt{2}}\left[\frac{1}{\sqrt{2}}\left(-\left\langle\leftrightarrow_{1}\right|\left\langle\uparrow_{2} \mid \Psi_{12}\right\rangle+\left\langle\leftrightarrow_{2}\right|\left\langle\uparrow_{1} \mid \Psi_{12}\right\rangle\right)\right]= \\
= & -\frac{1}{\sqrt{2}}\left[\left\langle\Psi_{12}^{(-)} \mid \Psi_{12}\right\rangle\right]
\end{aligned}
$$

Se o estado dos fótons 1 e $2\left(\left|\Psi_{12}\right\rangle\right)$ for o estado (4.26) da base de Bell( ou esse estado a menos de uma fase), (4.27) é igual a $\pm \frac{1}{\sqrt{2}}$. Segundo um dos Postulados da Mecânica Quântica, isso implica que a probabilidade do estado $\left|\Psi_{12}\right\rangle$ ser o vetor (4.26) da base de Bell é $\frac{1}{4}$. No experimento, esse evento em particular ocorre toda vez que os detectores $f 1$ e $f 2$ em Alice detectam simultaneamente a presença de um fóton, ou "clicam". De acordo com o protocolo de Bennett et al, o estado do fóton 3, por ser emaranhado com o fóton 2 , é instantaneamente projetado no estado (4.18) toda vez que o estado $\left|\Psi_{12}\right\rangle$ é do tipo (4.26).

\subsubsection{A estação de Bob e os resultados obtidos}

Após a detecção simultânea de fótons em $f 1$ e $f 2$, Alice avisa a Bob, através de canais clássicos, que detectou o estado 4.26. Bob, então, checa o estado de polarização do fóton 3 que, teoricamente, encontra-se no mesmo estado (4.18) do fóton 1 antes da realização da medida por Alice.

A verificação do estado do fóton 3 é realizada, na prática, por Bob através do uso de um PBS, que reflete estados de polarização vertical e transmite estados horizontais. O detector $d 1$ está posicionado após o PBS de tal forma que recebe fótons com polarização horizontal, e o detector $d 2$ fótons com polarização vertical.

Se o estado de polarização inicial do fotón 1 é vertical, o teletransporte ocorre toda vez que os detectores $f 1 f 2 d 1$ detectam simultaneamente fótons, o que implica na não ocorrência de detecção simultânea de fótons nos detectores $f 1 f 2 d 2$. Entretanto, se o estado inicial a ser teletransportado do fóton 1 é horizontal, a verificação do teletransporte é feita através da detecção simultânea de fótons em $f 1 f 2 d 2$ e da não-detecção simultânea em $f 1 f 2 d 1$. A base de estados polarizados utilizada no experimento foram os estados de polarização +45 graus e -45 graus (superposições dos estados $\leftrightarrow$ e $\uparrow$ ) e os resultados desse experimento são exibidos, em caráter ilustrativo, na Figura 4.4 . 

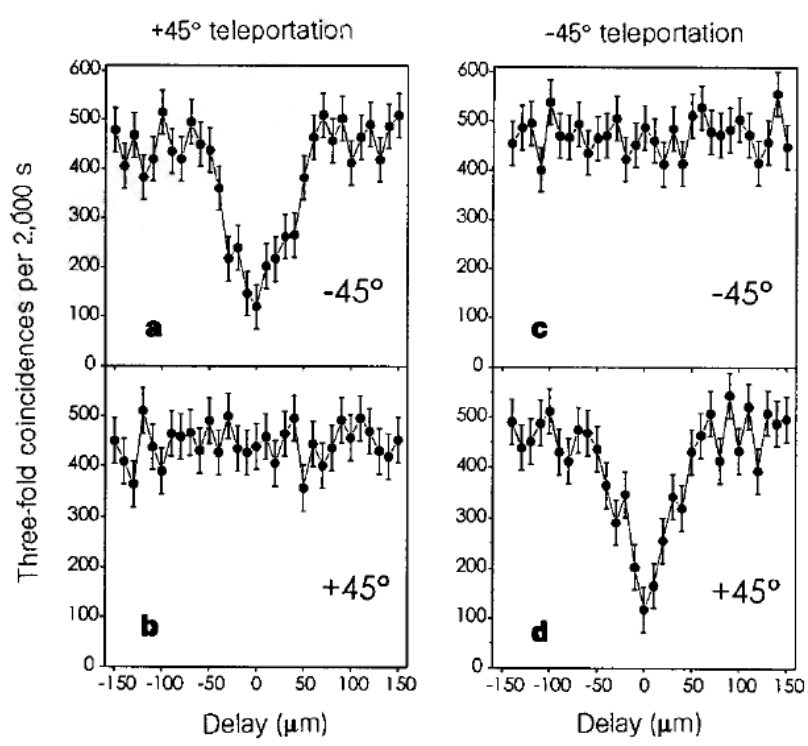

Figura 4.4: Figura do artigo [39] com os resultados experimentais obtidos pelos autores Bouwmeester et al na primeira implementação experimental do teletransporte quântico.

\subsection{Teletransporte em variáveis contínuas}

Os primeiros esforços visando aplicar a ideia do teletransporte foram feitos no contexto de variáveis discretas com qubits, dado que o próprio protocolo de Bennett et al. refere-se a sistemas desse tipo. Entretanto, conforme demonstrado no trabalho de S.L. Braunstein e H.J. Kimble [52], o teletransporte também pode ser realizado em sistemas cujos observáveis apresentam um espectro contínuo de autovalores, ditos de variáveis contínuas.

Assim como a informação quântica pode ser codificada, por exemplo, nos estados de polarização de um fóton, na forma conhecida como qubit, ela também pode ser codificada em termos dos modos quantizados da luz, que matematicamente são equivalentes aos osciladores harmônicos quantizados. Esta forma alternativa de codificar a informação quântica é denominada de qumodes e a presente seção refere-se a implementação do teletransporte quântico usando esses novos tipos de objetos quânticos.

Dentro dessa perspectiva, os estados coerentes da luz surgem naturalmente como candidatos à implementação do teletransporte pois, além da luz ser um meio comumente usado para a transmissão de informação e de ampla utilização tecnológica, ela interage fracamente com o ambiente que a circunda, o que dificulta o processo de decoerência. 
Conforme descrito na seção 4.4, o teletransporte de um qubit de informação é alcançado, no caso do experimento [39], somente quando o estado do par emaranhado $1-2$ for do tipo $\left|\Psi_{12}^{(-)}\right\rangle$, um dos vetores da base de Bell, e isso só ocorre com probabilidade $\frac{1}{4}$. Entretanto, o uso de variáveis contínuas no teletransporte torna possível que qualquer estado seja sempre teletransportado ou "incondicionalmente", como afirma A. Furusawa, J.L. Sørensen, S. L. Braunstein, C.A. Fuchs, H.J. Kimble e E. S. Polzik no trabalho entitulado "Teletransporte quântico incondicional" [7] publicado em 1998.

Esse trabalho foi a primeira implementação experimental do teletransporte em variáveis contínuas já realizada em que usou-se especificamente os estados com excesso de ruído da luz como os estados a serem teletransportados. Além da sua importância histórica na questão da transmissão de informação quântica, o esquema experimental de [7] serviu de base para a proposta do teletransporte bicolor que está sendo realizada no LMCAL.

Essa seção aborda os aspectos teóricos fundamentais envolvidos na realização do teletetransporte em variáveis contínuas de [7] e serão novamente retomados no contexto do capítulo 5 onde será abordado sobre o teletransporte bicolor.

O esquema utilizado em [7] para teletransportar um estado com excesso de ruído do campo eletromagnético é visto na Figura 4.5 e consiste essencialmente de uma fonte de estados EPR e de três estações operadas por Victor, Alice e Bob. Tais estações são responsáveis pela preparação, envio e reconstrução da informação contida nas flutuações quânticas do estado que se deseja teletransportar.

Victor prepara o estado e o envia para Alice, que realiza medidas sobre o estado e envia os resultados para Bob via canais clássicos de comunicação. Bob reconstrói o estado com o auxílio do canal quântico compartilhado com Alice. A discussão detalhada de como cada estação opera é feita a seguir.

Por serem observáveis análogos à posição e ao momento, as quadraturas do campo $\hat{X}_{\phi}$ e $\hat{Y}_{\phi}$, seguindo a notação original do artigo [7], serão designados de agora em diante por $\hat{x}$ e $\hat{p}$ respectivamente.

\subsubsection{Fonte de estados emaranhados}

Os estados emaranhados utilizados no experimento são produzidos através da combinação, em um BS, de dois feixes cujos os estados são vácuos comprimidos. Esses estados são [33, 53] gerados em um oscilador paramétrico ótico (OPO), operando em um regime diferente do nosso, abaixo do limiar de oscilação, cujas fases são devidamente controladas para que eles estejam 


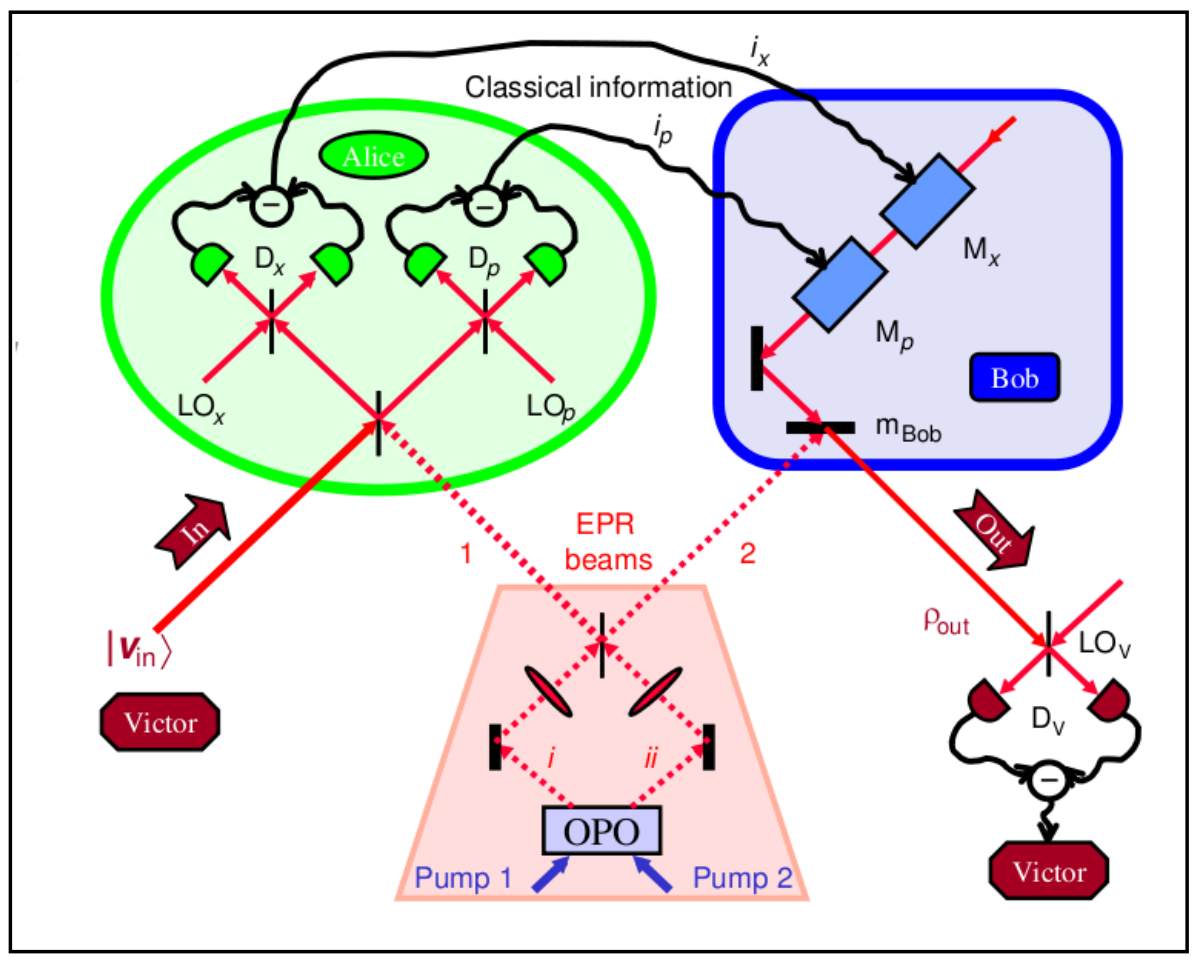

Figura 4.5: Figura da referência [7] do esquema experimental utilizado para teletransportar um estado coerente. As três estações envolvidas no processo são designadas por Victor, Alice e Bob. Victor envia o estado coerente para Alice e ela teletransporta o estado para Bob via canais quântico e clássicos de comunicação. O canal quântico é representado pelo par de feixes $1-2$, gerados pelo OPO operando abaixo do limiar. Os canais clássicos são os moduladores eletro-óticos, denotados por $M_{x}$ e $M_{p}$, e os cabos que transportam as informações contidas nas correntes elétricas $i_{x}$ e $i_{p}$. 
comprimidos em quadratura, um comprimido na quadratura $\hat{x}$ e o outro comprimido na quadratura $\hat{p}$.

Devido às propriedades de atuação do BS nos campos incidentes, ele pode ser utilizado para produzir estados emaranhados. A Figura 4.6 é uma ampliação da Figura 4.5 e mostra como ocorre a geração de estados emaranhados a partir da incidência de dois campos com estados de vácuo comprimido, representados na figura por $\hat{a}_{1}$ e $\hat{a}_{2}$.

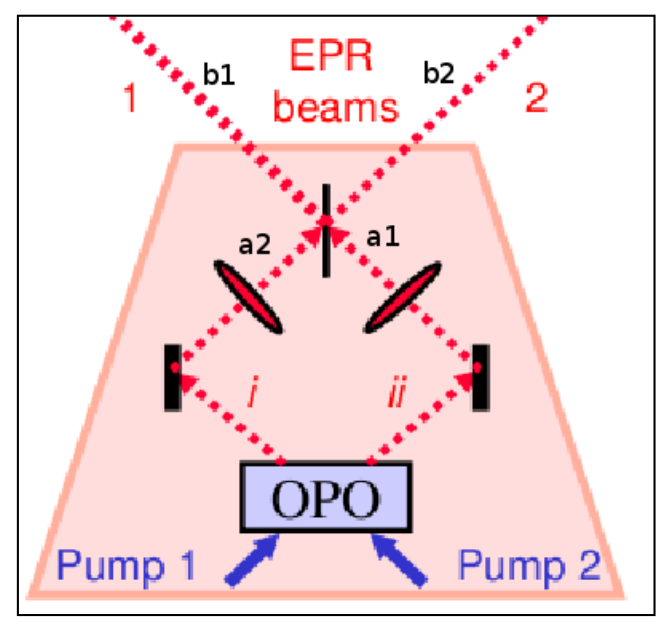

Figura 4.6: Ampliação da Figura 4.5 modificada. Fonte de estados emaranhados do experimento [7]. Os dois estados de vácuo comprimido dos campos $\hat{a}_{1}$ e $\hat{a}_{2}$, ao atravessar o BS, produzem os campos com estados emaranhados de vácuo comprimido $\hat{b}_{1}$ e $\hat{b}_{2}$. As elipses em vermelho representam artisticamente as elipses de ruído dos estados de vácuo dos campos $\hat{a}_{1}$ e $\hat{a}_{2}$, comprimidos nas quadraturas $\hat{x}$ e $\hat{p}$.

Os dois estados de vácuo comprimido, um na quadratura $\hat{x}$, e o outro na quadratura $\hat{p}$, são escritos de acordo com a equação (2.38), em que a compressão na quadratura $\hat{x}$ ocorre quando $\phi=0$, e a compressão em $\hat{p}$ quando $\phi=\pi[33,53]$ :

$$
\begin{aligned}
& \hat{a}_{1}=\hat{a}_{1}^{(0)} \cosh (r)+\hat{a}_{1}^{\dagger(0)} \operatorname{senh}(r), \\
& \hat{a}_{2}=\hat{a}_{2}^{(0)} \cosh (r)-\hat{a}_{2}^{\dagger(0)} \operatorname{senh}(r)
\end{aligned}
$$

A notação $\hat{a}^{(0)}$ serve só para indicar que se trata de um campo cujo estado é vácuo.

Os campos emaranhados $\hat{b}_{1}$ e $\hat{b}_{2}$ são produzidos através da passagem dos campos $\hat{a}_{1}$ e $\hat{a}_{2}$ pelo BS e são escritos como: 


$$
\begin{aligned}
& \hat{b}_{1}=\frac{1}{\sqrt{2}}\left(\hat{a}_{1}+\hat{a}_{2}\right) \\
& \hat{b}_{2}=\frac{1}{\sqrt{2}}\left(\hat{a}_{1}-\hat{a}_{2}\right)
\end{aligned}
$$

Substituindo (4.28) e (4.29) nas equações (4.30) e (4.31), obtém-se $\hat{b}_{1}$ e $\hat{b}_{2}$ em termos de $\hat{a}_{1}$ e $\hat{a}_{2}$ :

$$
\begin{aligned}
& \hat{b}_{1}=\frac{1}{\sqrt{2}}\left[\left(\hat{a}_{1}^{(0)}+\hat{a}_{2}^{(0)}\right) \cosh (r)+\left(\hat{a}_{1}^{\dagger(0)}-\hat{a}_{2}^{\dagger(0)}\right) \operatorname{senh}(r)\right] \\
& \hat{b}_{2}=\frac{1}{\sqrt{2}}\left[\left(\hat{a}_{1}^{(0)}-\hat{a}_{2}^{(0)}\right) \cosh (r)+\left(\hat{a}_{1}^{\dagger(0)}+\hat{a}_{2}^{\dagger(0)}\right) \operatorname{senh}(r)\right]
\end{aligned}
$$

Como é sabido, os operadores criação e aniquilação de um dado campo não são observáveis, entretanto as suas quadraturas $\hat{x}$ e $\hat{p}$ são grandezas observáveis, e dessa forma, sujeitas a medições. Para todos os campos envolvidos no experimento, é necessário calcular as quadraturas correspondentes.

De (4.32), é possível calcular a quadratura $\hat{x}_{1}$ do campo $\hat{b}_{1}$. Simplificandose a expressão obtida, temos:

$$
\hat{x}_{1}=\hat{b}_{1}^{\dagger}+\hat{b}_{1}=\frac{1}{\sqrt{2}}\left[e^{+r} \hat{x}_{1}^{(0)}+e^{-r} \hat{x}_{2}^{(0)}\right]
$$

De forma análoga ao cálculo anterior, é obtida a quadratura $\hat{p}_{1}$ do mesmo campo $\hat{b}_{1}$ :

$$
\hat{p}_{1}=-i\left(\hat{b}_{1}-\hat{b}_{1}^{\dagger}\right)=\frac{1}{\sqrt{2}}\left[e^{-r} \hat{p}_{1}^{(0)}+e^{+r} \hat{p}_{2}^{(0)}\right]
$$

Utilizando o mesmo procedimento descrito, encontra-se as quadraturas para o outro modo $\hat{b}_{2}$, emaranhado com $\hat{b}_{1}$ :

$$
\begin{aligned}
& \hat{x}_{2}=\frac{1}{\sqrt{2}}\left[e^{+r} \hat{x}_{1}^{(0)}-e^{-r} \hat{x}_{2}^{(0)}\right] \\
& \hat{p}_{2}=\frac{1}{\sqrt{2}}\left[e^{-r} \hat{p}_{1}^{(0)}-e^{+r} \hat{p}_{2}^{(0)}\right]
\end{aligned}
$$

Os feixes, cujas quadraturas foram calculadas, fazem parte do canal quântico do processo. O feixe representado por 1 está asssociado ao campo $\hat{b}_{1}$ e é entregue a Alice, enquanto que o feixe 2 , associado ao campo $\hat{b}_{2}$, é entregue a Bob. 


\subsubsection{A "medida de Bell" em Alice}

Victor é a estação que prepara o estado do campo que será teletransportado e verifica, depois, a fidelidade do processo. Ele cria um estado e envia para Alice iniciar o protocolo. O campo associado a esse estado é denotado na Figura 4.7 por $\hat{a}_{i n}$, cujas quadraturas são $\left(\hat{x}_{i n}, \hat{p}_{i n}\right)$.

O estado do campo $\hat{a}_{i n}$ a ser teletransportado, de quadraturas $\left(\hat{x}_{i n}, \hat{p}_{i n}\right)$, é enviado para Alice e combinado em um BS 50 : 50 com o feixe $\hat{b}_{1}$ de quadraturas $\left(\hat{x}_{1}, \hat{p}_{1}\right)$, emaranhado com o feixe $\hat{b}_{2}$, de posse de Bob.

Esses feixes são combinados no BS de forma a produzir outros, denotados por $\hat{a}_{u}$ e $\hat{a}_{v}$ e vistos, esquematicamente, na Figura 4.7:

$$
\begin{aligned}
& \hat{a}_{u}=\frac{1}{\sqrt{2}}\left(\hat{a}_{i n}-\hat{b}_{1}\right) \\
& \hat{a}_{v}=\frac{1}{\sqrt{2}}\left(\hat{a}_{i n}+\hat{b}_{1}\right)
\end{aligned}
$$

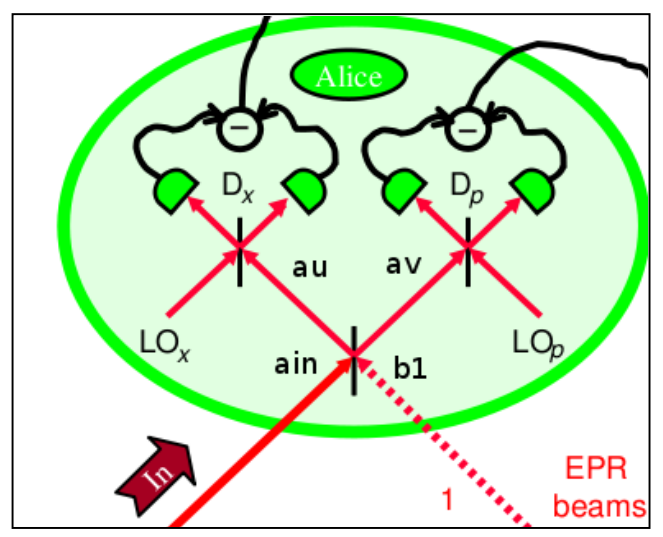

Figura 4.7: Ampliação da Figura 4.5 modificada que ilustra a medida de Bell em Alice. Os campos incidentes $\hat{a}_{i n}$ e $\hat{b}_{1}$ são combinados no BS. Como resultado desse processo, surgem os campos $\hat{a}_{u}$ e $\hat{a}_{v}$, cujas quadraturas são $\left(\hat{x}_{u}, \hat{p}_{u}\right)$ e $\left(\hat{x}_{v}, \hat{p}_{v}\right)$ respectivamente. Alice mede as quadraturas $\hat{x}_{u}$ e $\hat{p}_{v}$ por meio da técnica de detecção homodina balaceada. Os detectores homodinos são denotados por $D_{x}$ e $D_{p}$ e seus respectivos osciladores locais por $L O_{x}$ e $L O_{p}$.

Calcula-se a quadratura $\hat{x}_{u}$ do campo $\hat{a}_{u}$ :

$$
\hat{x}_{u}=\left(\hat{a}_{u}+\hat{a}_{u}^{\dagger}\right)=\frac{1}{\sqrt{2}}\left[\left(\hat{a}_{i n}+\hat{a}_{i n}^{\dagger}\right)-\left(\hat{b}_{1}+\hat{b}_{1}^{\dagger}\right)\right]
$$


Reescrevendo-se (4.40) em termos das quadraturas dos campos de entrada no BS, $\hat{b}_{1}$ e $\hat{a}_{i n}$, obtém-se:

$$
\hat{x}_{u}=\frac{1}{\sqrt{2}}\left(\hat{x}_{i n}-\hat{x}_{1}\right)
$$

De forma análoga, determina-se a quadratura $\hat{p}_{u}$,

$$
\hat{p}_{u}=-i\left(\hat{a}_{u}-\hat{a}_{u}^{\dagger}\right)=\frac{1}{\sqrt{2}}\left[-i\left(\hat{a}_{i n}-\hat{a}_{i n}^{\dagger}\right)-i\left(-\hat{b}_{1}+\hat{b}_{1}^{\dagger}\right)\right]=\frac{1}{\sqrt{2}}\left(\hat{p}_{i n}-\hat{p}_{1}\right)
$$

Repetindo-se o mesmo procedimento descrito, calculam-se também as quadraturas $\left(\hat{x}_{v}, \hat{p}_{v}\right)$ do campo $\hat{a}_{v}$ :

$$
\begin{gathered}
\hat{x}_{v}=\frac{1}{\sqrt{2}}\left[\left(\hat{a}_{i n}+\hat{a}_{i n}^{\dagger}\right)+\left(\hat{b}_{1}+\hat{b}_{1}^{\dagger}\right)\right]=\frac{1}{\sqrt{2}}\left(\hat{x}_{i n}+\hat{x}_{1}\right) \\
\hat{p}_{v}=\frac{1}{\sqrt{2}}\left[-i\left(\hat{a}_{i n}-\hat{a}_{i n}^{\dagger}\right)-i\left(\hat{b}_{1}-\hat{b}_{1}^{\dagger}\right)\right]=\frac{1}{\sqrt{2}}\left(\hat{p}_{i n}+\hat{p}_{1}\right)
\end{gathered}
$$

Alice usa a técnica da detecção homodina balanceada, descrita no capítulo 3 , para medir as quadraturas $\hat{x}_{u}$ e $\hat{p}_{v}$. Os detectores homodinos utilizados são representados na Figura 4.7 por $D_{x}$ e $D_{p}$ e os respectivos osciladores locais por $L O_{x}$ e $L O_{p}$.

No momento da medida das quadraturas, são produzidas duas correntes elétricas $\left(i_{x}\right.$ e $\left.i_{p}\right)$ que são amplificadas e transformadas em sinais de tensão elétrica, resultando em dois processos estocásticos clássicos. São esses processos que representam a informação clássica que Alice envia à Bob via cabos.

Obviamente, não faz sentido, nesse caso, falar em projetar na base de Bell, porém vale a analogia. Dessa forma, essas medidas correspondem à medida de Bell no contexto do teletransporte em variáveis contínuas [33].

A "medida de Bell" realizada por Alice acopla os campos $\hat{a}_{i n}$ e $\hat{b}_{2}$, e, no caso ideal, em que o parâmetro de compressão $r$ tende ao infinito, Alice não ganha, com a medida, qualquer informação sobre a amplitude e a fase do estado a ser teletransportado $\left(\hat{x}_{i n}, \hat{p}_{i n}\right)$ pois, no limite de $r \rightarrow \infty$, as equações (4.34) e (4.35) resultam em:

$$
\begin{array}{lll}
\lim _{r \rightarrow \infty} & \hat{x}_{1} \rightarrow \infty \\
\lim _{r \rightarrow \infty} & \hat{p}_{1} \rightarrow \infty
\end{array}
$$

Como a quadratura referente à amplitude do campo $\hat{a}_{u}$, denotada por $\hat{x}_{u}$ e a quadratura referente à fase do campo, $\hat{p}_{v}$, medidas por Alice, são fornecidas 
pelas equações (4.41) e (4.44), então obtém-se também na condição ideal de compressão:

$$
\begin{aligned}
& \lim _{r \rightarrow \infty}\left\langle\Delta^{2} \hat{x}_{u}\right\rangle \rightarrow \infty \\
& \lim _{r \rightarrow \infty}\left\langle\Delta^{2} \hat{p}_{v}\right\rangle \rightarrow \infty
\end{aligned}
$$

De acordo com o teorema da não-clonagem, um estado quântico qualquer não pode ser copiado. Assim, quanto mais Alice obtém informação sobre o estado a ser teletransportado menor será a fidelidade do processo de teletransporte.

Como os feixes $\hat{b}_{1}$ e $\hat{b}_{2}$ estão emaranhados, a medida de Bell em Alice muda o estado do campo $\hat{b}_{2}$, ou colapsa ${ }^{6}$ [7], para um outro estado condicionado às fotocorrentes $i_{x}$ e $i_{p}[7]$, segundo veremos na próxima seção.

\subsubsection{A reconstrução do estado em Bob}

Alice envia os resultados de suas medidas para Bob via canais clássicos de comunicação. Os cabos vistos na Figura 4.8 levam essas informações para Bob que reconstrói o estado preparado por Victor para o teletransporte.

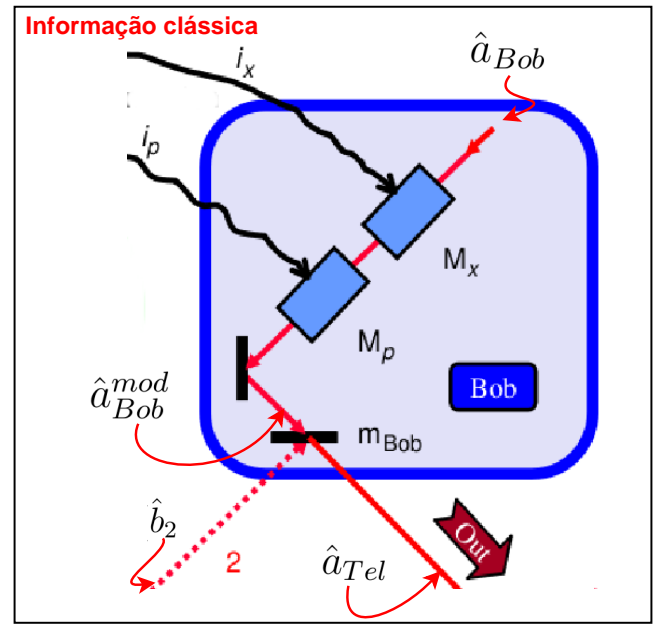

Figura 4.8: Ampliação da Figura 4.5 modificada que ilustra a estação de Bob. Bob recebe o feixe $\hat{b}_{2}$, emaranhado com $\hat{b}_{1}$ dado a Alice, e usa as medidas enviadas por Alice, via cabos, para modular o feixe coerente, denotado na figura por $\hat{a}_{B o b}$. Esse feixe modulado é representado na figura por $\hat{a}_{B o b}^{\text {mod }}$. No espelho $m_{B o b}$, o feixe $\hat{a}_{B o b}^{\text {mod }}$ juntamente com o feixe $\hat{b}_{2}$ são combinados, resultando no campo $\hat{a}_{T e l}$. O coeficiente de reflexão do espelho é 0,99.

\footnotetext{
${ }^{6}$ O termo "colapsar" ou "reduzir o pacote de onda" é muito usado nos artigos e significa a mudança de um estado quântico induzida pelo processo de observação [50].
} 
As informações contidas inicialmente nas correntes $i_{x}$ e $i_{p}$, que são amplificadas e transformadas em tensões, são proporcionais às medidas das quadraturas $\hat{x}_{u}$ e $\hat{p}_{v}$, respectivamente, e Bob as usa para modular a amplitude e a fase de um feixe coerente [33] usado como base para a reconstrução do estado enviado por Alice. Esse feixe usado por Bob é denotado por $\hat{a}_{B o b}$ e o feixe já modulado por Bob é denominado $\hat{a}_{B o b}^{\text {mod }}$, vistos na Figura 4.8.

De acordo com o capítulo 2, o estado coerente é o estado com o menor ruído associado às suas quadraturas e exatamente por causa dessa característica que ele é usado por Bob para reconstruir o estado teletransportado.

As modulações realizadas por Bob sobre as quadraturas $\hat{x}$ e $\hat{p}$ do campo coerente $\hat{a}_{B o b}$ são realizadas através dos moduladores eletro-óticos (EOM), representados por $M_{x}$ e $M_{p}$. Esses dispositivos juntamente com os cabos que carregam as tensões elétricas, fazem parte do canal clássico do sistema. Os princípios físicos envolvidos no funcionamento dos EOMs, assim como a forma de caracterizá-los, serão minuciosamente descritos no capítulo 6 .

As quadraturas $\hat{x}_{2}$ e $\hat{p}_{2}$ do campo $\hat{b}_{2}$ dado a Bob, podem ser reescritas $[33,53]$ usando-se as equações $(4.41),(4.42),(4.43),(4.44)$ e as equações (4.34), (4.35), (4.36) e (4.37), conforme observado a seguir.

Como o campo $\hat{b}_{2}$ é emaranhado com o campo $\hat{b}_{1}$, é conveniente reescrever suas quadraturas $\hat{x}_{2}$ e $\hat{p}_{2}$ somente em termos das quadraturas do campo cujo estado se deseja teletransportar $\left(\hat{x}_{i n}, \hat{p}_{i n}\right)$, das quadraturas do campo de vácuo comprimido $\left(\hat{x}_{2}^{(0)}, \hat{p}_{1}^{(0)}\right)$ e as quadraturas dos campos $\hat{a}_{u}$ e $\hat{a}_{v}$, que Alice mede com a detecção homodina, no caso, $\hat{x}_{u}$ e $\hat{p}_{v}$.

Calculando-se $\left(\hat{x}_{1}-\hat{x}_{2}\right)$, dadas as equações (4.34) e (4.36), temos:

$$
\left(\hat{x}_{1}-\hat{x}_{2}\right)=\sqrt{2} e^{-r} \hat{x}_{2}^{(0)}
$$

Sabe-se, de (4.41), que $\hat{x}_{1}$ é igual a:

$$
\hat{x}_{1}=\hat{x}_{i n}-\sqrt{2} \hat{x}_{u}
$$

É conveniente escrever $\hat{x}_{2}$ em termos de $\left(\hat{x}_{1}-\hat{x}_{2}\right)$ :

$$
\hat{x}_{2}=-\left(\hat{x}_{1}-\hat{x}_{2}\right)+\hat{x}_{1}
$$

Substituindo-se (4.49) em (4.51), resulta em:

$$
\hat{x}_{2}=-\sqrt{2} e^{-r} \hat{x}_{2}^{(0)}+\hat{x}_{i n}-\sqrt{2} \hat{x}_{u}
$$

Para a quadratura $\hat{p}_{2}$, calcula-se $\left(\hat{p}_{1}+\hat{p}_{2}\right)$, dadas as equações $(4.35) \mathrm{e}$ (4.37):

$$
\left(\hat{p}_{1}+\hat{p}_{2}\right)=\sqrt{2} e^{-r} \hat{p}_{1}^{(0)}
$$


Sabe-se, de (4.44), que $\hat{p}_{1}$ é igual a:

$$
\hat{p}_{1}=\sqrt{2} \hat{p}_{v}-\hat{p}_{i n}
$$

Substituindo-se (4.53) e (4.54) em:

$$
\hat{p}_{2}=\left(\hat{p}_{1}+\hat{p}_{2}\right)-\hat{p}_{1},
$$

resulta na quadratura $\hat{p}_{2}$ que depende somente das quadraturas $\hat{p}$ dos campos $\hat{a}_{i n}$ (campo cujo estado é teletransportado), $\hat{a}_{1}^{(0)}$ (vácuo comprimido) e $\hat{a}_{v}$ (um dos campos resultante da combinação de $\hat{a}_{i n}$ e $\hat{b}_{1}$ no BS em Alice):

$$
\hat{p}_{2}=\hat{p}_{i n}+\sqrt{2} e^{-r} \hat{p}_{1}^{(0)}-\sqrt{2} \hat{p}_{v}
$$

Usando as relações de BS, é possível escrever o campo $\hat{a}_{T e l}$ em função dos campos de entrada $\hat{b}_{2}$ e $\hat{a}_{B o b}^{\text {mod }}$. São as quadraturas do campo $\hat{a}_{T e l}$ que serão medidas pela estação de Victor para a verificação da fidelidade do processo de teletransporte.

Dado que o coeficiente de reflexão de $m_{B o b}$, usado em [7] é de 0,99, obtémse para $\hat{a}_{T e l}$ :

$$
\hat{a}_{T e l}=\sqrt{0,99} \hat{b}_{2}+\sqrt{0,01} \hat{a}_{B o b}^{\text {mod }}
$$

A partir da expressão (4.57), calcula-se ${ }^{7}$ as suas respectivas quadraturas $\hat{x}_{T e l}$ e $\hat{p}_{T e l}$. A quadratura $\hat{x}_{T e l}$ é dada por:

$$
\hat{x}_{T e l}=\left(\hat{b}_{2}+\hat{b}_{2}^{\dagger}\right)+0,1\left(\hat{a}_{B o b}^{\text {mod }}+\hat{a}_{B o b}^{\dagger m o d}\right)
$$

Substituindo-se a equação (4.33) na equação (4.58) e simplificando, resulta, finalmente, na expressão para a quadratura $\hat{x}_{T e l}$ :

$$
\hat{x}_{T e l}=\frac{1}{\sqrt{2}}\left(e^{+r} \hat{x}_{1}^{(0)}-e^{-r} \hat{x}_{2}^{(0)}\right)+0,1 \hat{x}_{T e l}=\hat{x}_{2}+0,1 \hat{x}_{B o b}^{m o d}
$$

O mesmo raciocínio é aplicado no cálculo da quadratura $\hat{p}_{T e l}$ que fornece a expressão:

$$
\hat{p}_{T e l}=\frac{1}{\sqrt{2}}\left[e^{-r} \hat{p}_{1}^{(0)}-e^{+r} \hat{p}_{2}^{(0)}\right]+0,1 \hat{p}_{B o b}^{m o d}=\hat{p}_{2}+0,1 \hat{p}_{B o b}^{\text {mod }}
$$

O campo $\hat{b}_{2}$ de Bob, como já mencionado, colapsa em um estado que difere do estado de entrada $\left(\hat{x}_{i n}, \hat{p}_{i n}\right)$, preparado por Victor, somente de um deslocamento no espaço de fase [7]. Assim, após receber as medidas

\footnotetext{
${ }^{7}$ Usando-se a aproximação $\sqrt{0,99}=1$.
} 
clássicas das quadraturas $\hat{x}_{u}$ e $\hat{p}_{v}$ de Alice, Bob desloca seu modo, de forma que resulta nas quadraturas $\hat{x}_{T e l}$ e $\hat{p}_{T e l}$. A Figura 4.9 exibe o espaço de fase com os estados coerentes e o deslocamento realizado por Bob.

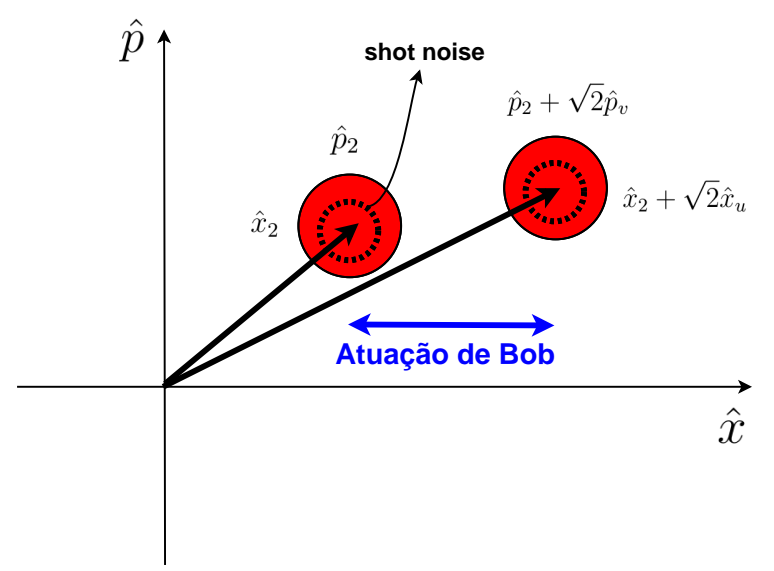

Figura 4.9: Representação no espaço de fase do estado coerente inicial do campo $\hat{a}_{B o b}$ e o deslocamento sofrido por ele pela atuação de Bob. Os círculos, em vermelho, representam os estados coerentes dos campos e o círculo pontilhado representa o estado do vácuo.

De acordo com as equações (4.50) e (4.54), os termos $\sqrt{2} \hat{x}_{u}$ e $\sqrt{2} \hat{p}_{v}$ estão relacionados às medidas de Bell realizadas por Alice e, são responsáveis, a menos de um ganho $[33,53]$, pelo deslocamento feito por Bob das quadraturas $\hat{x}_{2}, \hat{p}_{2}$. As expressões (4.63) e (4.64) são dadas considerando que o ganho é 1. Isso significa que a informação contida nas fotocorrentes $i_{x}$ e $i_{p}$ foram completamente utilizadas por Bob no processo de deslocamento.

Das equações (4.36) e (4.37), observa-se que o deslocamento nas quadraturas do campo $\hat{b}_{2}$, após a realização das medidas por Alice, é igual a:

$$
\begin{aligned}
& \hat{x}_{2}+\sqrt{2} \hat{x}_{u}=\hat{x}_{i n}-\sqrt{2} e^{-r} \hat{x}_{2}^{(0)} \\
& \hat{p}_{2}+\sqrt{2} \hat{p}_{v}=\hat{p}_{i n}+\sqrt{2} e^{-r} \hat{p}_{1}^{(0)}
\end{aligned}
$$

Substitundo-se as equações (4.59) e (4.60) nas equações acima, resulta nas quadraturas do campo $\hat{a}_{T e l}$. Essas quadraturas serão posteriormente medidas por Victor a fim de verificar a realização do teletransporte do estado inicialmente preparado por ele:

$$
\hat{x}_{T e l}=\hat{x}_{2}+0,1 \hat{x}_{B o b}^{m o d}=\hat{x}_{i n}-\sqrt{2} e^{-r} \hat{x}_{2}^{(0)}
$$




$$
\hat{p}_{T e l}=\hat{p}_{2}+0,1 \hat{p}_{\text {Bob }}^{\text {mod }}=\hat{p}_{\text {in }}+\sqrt{2} e^{-r} \hat{p}_{1}^{(0)}
$$

As equações (4.63) e (4.64) mostram que, se $r$ tender ao infinito, as quadraturas $\hat{x}_{T e l}$ e $\hat{p}_{T e l}$ são iguais as quadraturas $\hat{x}_{i n}$ e $\hat{p}_{i n}$ do campo $\hat{a}_{i n}$.

Um parâmetro de compressão tão grande implica em perfeito emaranhamento entre os campos $\hat{a}_{1}$ e $\hat{a}_{2}$, como isso, na prática, nunca é alcançado, é importante garantir a máxima compressão experimentalmente possível entre o par EPR de forma que o estado do campo teletransportado se aproxime do estado do campo inicialmente preparado por Victor.

No capítulo 6, veremos que construir uma fonte de estados emaranhados com a maior compressão de ruído possível foi o nosso maior desafio na implementação do teletransporte bicolor e que todo esse nosso esforço técnico será recompensado com um aumento na fidelidade do processo.

\subsection{4 "Quantum duties" e resultados}

Antes de ilustrar os resultados experimentais obtidos pelos autores Furusawa et al. em [7] no teletransporte de estados coerentes, consideremos o caso do mesmo esquema porém sem o par EPR, ou seja, na ausência de emaranhamento, caracterizando uma espécie de teletransporte clássico, que corresponde a $r=0$ nas equações (4.63) e (4.64).

Nesse situação, tanto na estação de Alice quanto na de Bob entram campos em estados de vácuo no lugar dos feixes EPR. A Figura 4.10 ilustra o esquema clássico do teletransporte adaptado de [7].

Dessa forma, cada quadratura do estado teletransportado classicamente acaba apresentando um ruído quântico com duas unidades de ruído de vácuo a mais comparada ao ruído do estado original preparado por Victor. Essas duas unidade a mais são chamadas de "quantum duties", ou, como os autores afirmam em [79], a mínima "tarifa" a ser paga quando se cruza a fronteira entre os domínios clássico e quântico. Como consequência disso, a fidelidade do teletransporte clássico é drasticamente reduzida, atingindo no máximo, $50 \%$.

Uma dessas contribuições vem de Alice ao tentar medir as quadraturas, simultaneamente, $\left(\hat{x}_{i n}\right.$ e $\left.\hat{p}_{i n}\right)$ e a outra vem de Bob ao tentar usar as informações clássicas obtidas de Alice para reconstruir um estado quântico enviado por Victor.

Como já mencionado, Alice utiliza o método de detecção homodina para medir as quadraturas $\hat{x}_{u}$ e $\hat{p}_{v}$, cujos os resultados consistem em flutuações nas fotocorrentes geradas nos detectores, que são transformadas em sinais de tensão através da amplificação em transimpedância. 


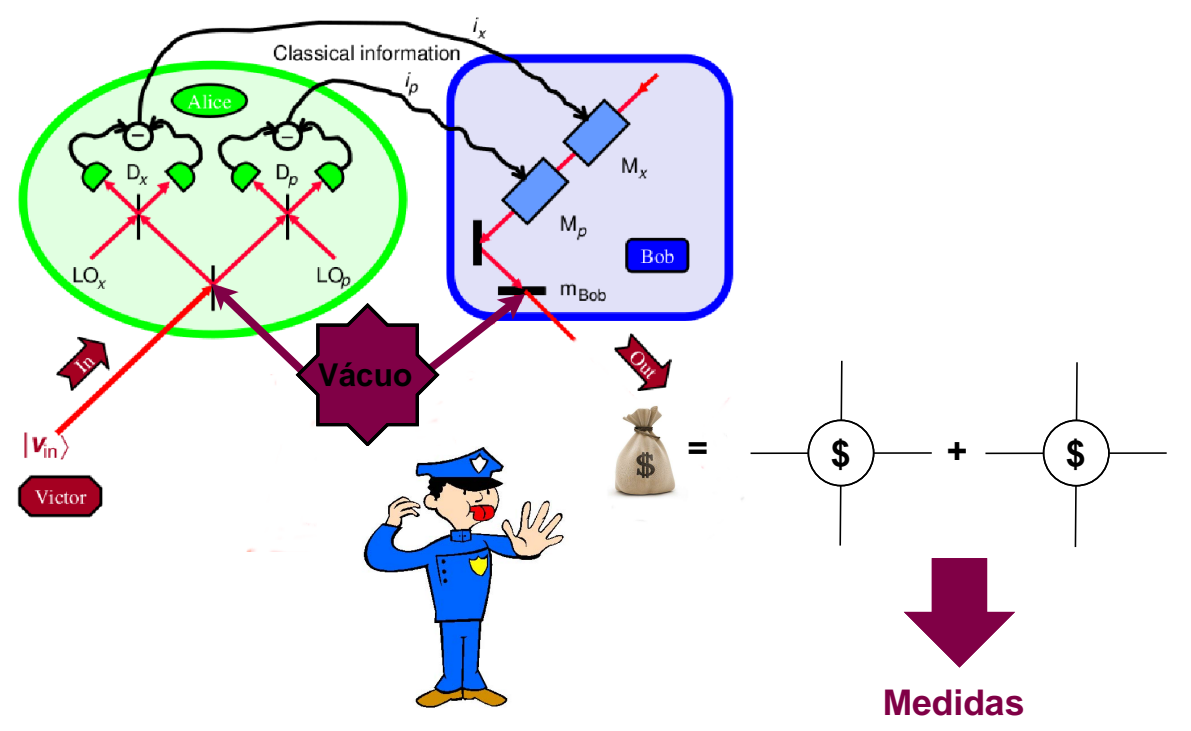

Figura 4.10: Figura artística adaptada da referência [7] meramente ilustrativa. Representa o mesmo esquema de teletransporte visto em [7], só que sem o emaranhamento dos feixes, caracterizando o teletransporte clássico. Nesse caso, entra campos em estado de vácuo, os "quantum duties", nas estações de Alice e Bob.

A Figura 4.11 mostra os resultados dessas medidas de tensão obtidos pelos autores Furusawa et al. $\mathrm{O}$ eixo horizontal do gráfico corresponde à fase do oscilador local $L O_{x}$, que varia no tempo, enquanto que o eixo vertical, denotado por $\Psi_{x}^{\text {Alice }}(\Omega)$ representa a densidade espectral da flutuação da tensão produzida pela amplificação da fotocorrente, em unidades de $d B$, geradas especificamente no detector homodino $D_{x}$, que mede a quadratura de amplitude $\hat{x}_{u}[7,33]$.

Victor prepara dois tipos de estados coerentes para o teletransporte: um estado de vácuo, para servir de calibração da medida, e um estado de vácuo deslocado ou estado coerente.

No caso em que o estado preparado por Victor é o estado de vácuo, o gráfico 4.11 fornece o nível da flutuação do ruído quântico para a medida de $\hat{x}_{u}$ referente a esse estado sem o par EPR, denotado por $\Phi_{0, x}^{\text {Alice }}(\Omega)$, e com o par presente presente no processo, denotado por $\Lambda_{x}^{\text {Alice }}(\Omega)[7]$.

O detalhe $B$ da Figura 4.11 mostra que o nível de ruído de $\Lambda_{x}^{\text {Alice }}(\Omega)$ com o par EPR é maior que o nível $\Phi_{0, x}^{\text {Alice }}(\Omega)$ sem o mesmo, uma vez que Alice perde informação do estado inicial com teletransporte quântico [33]. Conforme visto no limite (4.48), no caso ideal em que $r \rightarrow \infty$, o nível de ruído quântico deveria ser $\Lambda_{x}^{\text {Alice }}(\Omega) \rightarrow \infty$ [33]. 
No detalhe $A$ da mesma figura, é vista a medida da quadratura $\hat{x}$ em Alice, denotada por $\Psi_{x}^{\text {Alice }}(\Omega)$, referente a um estado coerente com excesso de ruído preparado por Victor [7]. Verifica-se que o valor máximo atingido é de aproximadamente $22 d B$ acima do ruído do "shot noise". Segundo a referência [33], esse valor é metade do valor referente ao estado coerente original que é de $25 d B$. Essa queda de $-3 d B$ [33] ocorre devido a combinação do feixe a ser teletransportado com o feixe que faz parte do par EPR no BS e a sua consequente divisão.

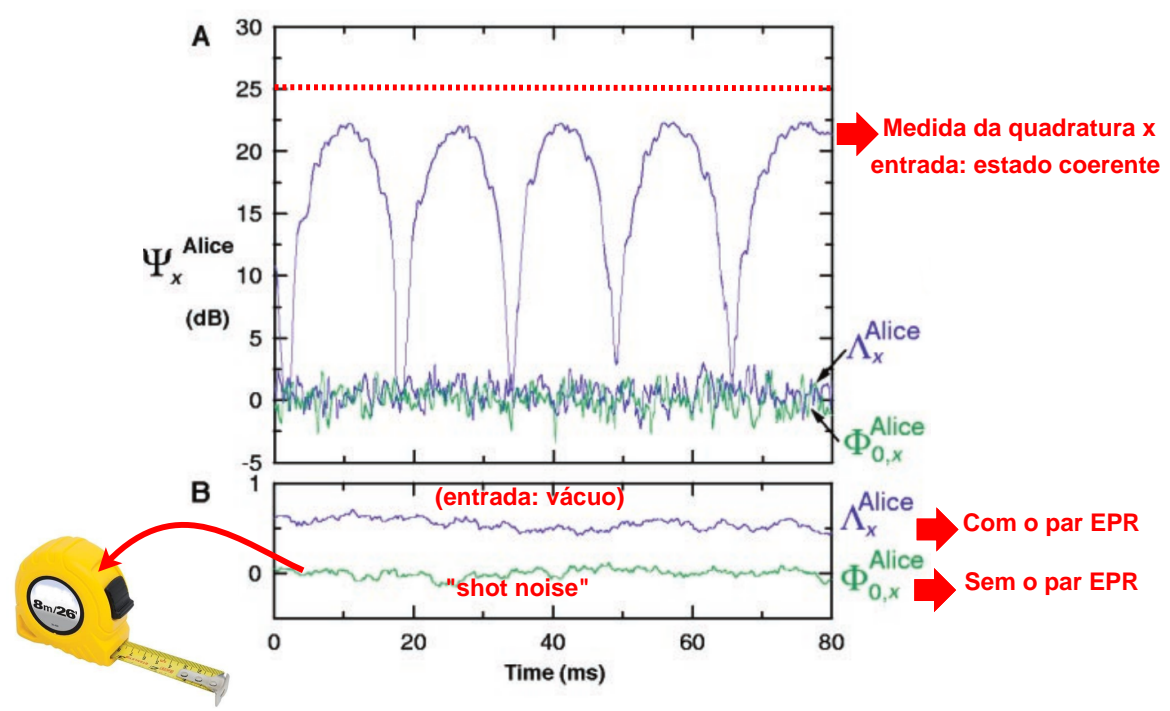

Figura 4.11: Figura adaptada da referência [7] com os resultados experimentais obtidos pelos autores Furusawa et al. que mostra a densidade espectral das flutuações das fotocorrentes obtidas por Alice no detector homodino $D_{x}$.

A Figura 4.12 mostra os resultados obtidos por Victor na verificação do teletransporte do estado de vácuo e do estado coerente $\left(\hat{x}_{i n}, \hat{p}_{i n}\right)$ preparado por ele.

Novamente, assim como na Figura 4.11, o eixo horizontal corresponde à fase do oscilador local de Victor, denotado na figura 4.5 por $L O_{v}$, e o eixo vertical as medidas das quadraturas ${ }^{8} \hat{x}_{t e l}$ e $\hat{p}_{t e l}$.

A curva $Y_{0}^{\text {Victor }}(\Omega)$ diz respeito ao teletransporte do estado de vácuo produzido por Victor sem a presença do par EPR, caracterizando o teletransporte clássico. A curva $\Lambda^{\text {Victor }}(\Omega)$ refere-se ao teletransporte do mesmo estado com a presença do par EPR ou emaranhamento. Observa-se, como

\footnotetext{
${ }^{8}$ Dada pela densidade espectral das flutuações das fotocorrentes associadas às quadraturas.
} 
esperado, que o nível de ruído na ausência de emaranhamento, $Y_{0}^{\text {Victor }}(\Omega)$, é três ${ }^{9}$ unidades de vácuo (aproximadamente $5 \mathrm{~dB}$ acima do "shot noise" ${ }^{10}$ ) maior que $\Lambda^{\text {Victor }}(\Omega)$.

O valor máximo da curva periódica refere-se à amplitude do estado coerente resultante do processo de teletransporte, aproximadamente $25 \mathrm{~dB}$ acima do nível $\Phi_{0}^{\text {Victor }}(\Omega)$, referente ao "shot noise" de $D_{v}[7]$.

A fidelidade observada pelos autores [7] foi de $0.48 \pm 0.03$ para o teletransporte sem o par EPR, teletransporte dito clássico, e $0.58 \pm 0.02$ com o par EPR, confirmando o caráter quântico do teletransporte, segundo a inequação (4.17).

Após esse primeiro trabalho [7] do teletransporte quântico usando qumodes, surgiram outros $[54,55,56]$ aprimorando cada vez mais o grau de fidelidade, destacando-se [56] com $F=0.81 \pm 0.01$, maior valor obtido até o momento, segundo a referência [33].

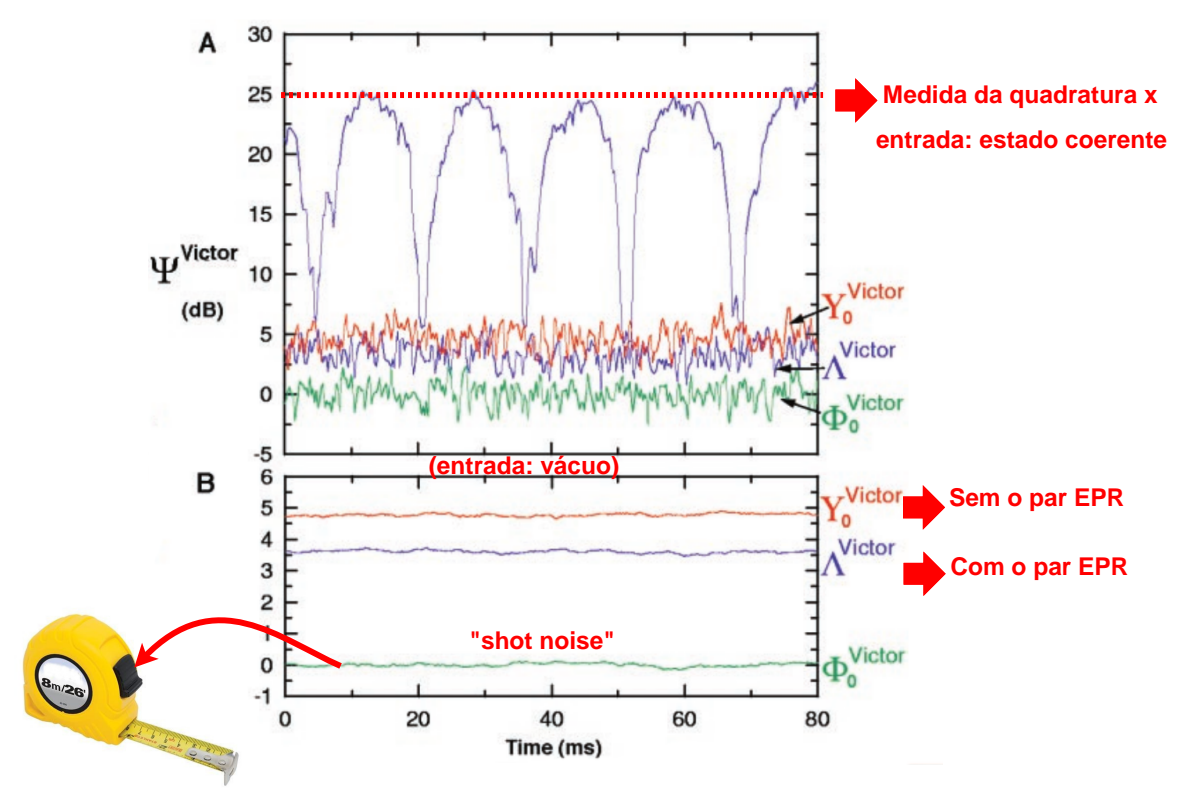

Figura 4.12: Figura adaptada da referência [7] com os resultados experimentais obtidos pelos autores Furusawa et al. que mostra a densidade espectral das flutuações das fotocorrentes obtidas por Victor.

\footnotetext{
${ }^{9}$ Uma unidade do estado enviado por Victor mais duas unidades de "quantum duties".

${ }^{10} \mathrm{O}$ ruído do "shot noise", ou unidade de vácuo(u.v), é a referência de medida, considerada igual a 1, logo, segundo a definição 3.45: $5 d B=10 \log (3 u . v)$
} 


\section{Capítulo 5}

\section{Proposta do teletransporte quântico bicolor}

Devido a importância do teletransporte em variáveis contínuas como meio de transmitir a informação de sistemas quânticos, propomos, nesse trabalho, um esquema experimental inovador usando um oscilador paramétrico ótico (OPO) operando em um regime onde dois feixes emaranhados nãodegenerados e intensos são gerados. Esse tipo de operação irá permitir, no futuro, a comunicação quântica entre sistemas que operam em diferentes frequências.

Nesse capítulo, abordaremos primeiro a motivação para a realização de um teletransporte desse tipo, chamado de "teletransporte bicolor", depois sobre as nossas duas propostas de implementação do protocolo e de que forma as estações Alice, Bob e Victor operam sobre os campos.

\subsection{Motivação da ideia do teletransporte bi- color}

Em um contexto mais geral, podemos afirmar que a manipulação da informação quântica em unidades isoladas, ou núcleos, e separadas umas das outras é um dos principais desafios atuais da comunidade científica que, futuramente, abrirá portas não só para aplicações tecnológicas impactantes como computadores quânticos ou implementações de esquemas de criptografia super seguros, como também para a possibilidade de investigação dos próprios fundamentos da Mecânica Quântica.

A conectividade entre essas unidades quânticas de processamento de informação, que formariam uma espécie de rede quântica [57], é o elemento chave dessa enorme empreitada e, sem dúvida, a luz é uma forte candidata a 
exercer o papel de canal por meio do qual os núcleos quânticos conectam-se à rede, pois os fótons interagem fracamente com o ambiente que os circunda.

A Figura 5.1, por exemplo, exibe uma representação artística de uma rede quântica em que as figuras coloridas referem-se a sistemas quânticos que operam em um regime de três cores, o que permite a conexão com outros sistemas e com uma unidade central de processamento, em cinza. Na Figura, as unidades de processamento são representadas por desenhos diversos para enfatizar a generalidade do sistema, podendo ser qualquer sitema físico.

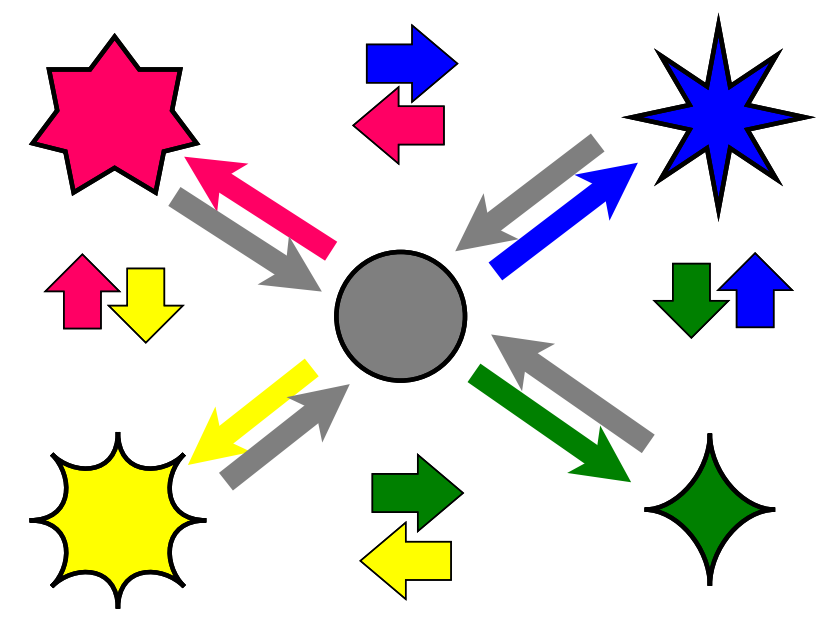

Figura 5.1: Representação artística de uma rede quântica, inspirada em uma figura da referência [57], e cujas unidades isoladas de processamento em rosa, azul, amarelo e verde comunicam-se entre si e com uma unidade central, em cinza, através da interação da luz com essas unidades em diferentes comprimentos de onda.

De forma esquemática, podemos resumir o funcionamento de uma rede quântica como uma rede que tanto gera a informação quântica quanto processa e armazena em seus núcleos quânticos, ou nós [57], que estão conectados entre si por canais quânticos que transportam a informação de um núcleo a outro [57].

Esses núcleos poderiam ser, por exemplo, ensembles atômicos que armazenariam a informação do sistema, em uma espécie de "memória quântica", comunicando-se com outros núcleos por meio da luz. Nesse caso, o uso do emaranhamento permitiria a comunicação entre diferentes ensembles atômicos através da transferência de informação em comprimentos de onda diferentes.

Um candidato natural para gerar feixes emaranhados intensos em cores distintas, que possa, por exemplo, interagir com os ensembles atômicos, é o OPO (oscilador paramétrico ótico), um dos sistemas mais utilizados em 
variáveis contínuas para geração de emaranhamento. Por ser sintonizável, o OPO permite a interação com sistemas que operam em diferentes faixas de frequência. Assim, é possível a comunicação entre ensembles atômicos de diferentes espécies, íons ou quantum dots, desde que o OPO seja projetado para trabalhar nas faixas de frequências desejadas.

Em um modo de operação particular, conforme veremos no próximo capítulo, o OPO gera feixes emaranhados não-degenerados em frequência e intensos que permitem usar a técnica da detecção auto-homodina para medir as quadraturas do campo, o que torna a montagem experimental menos complicada sem o uso de um oscilador local externo.

Tendo esse cenário em vista, e todo o conhecimento acumulado ao longos dos anos pelo nosso grupo sobre o OPO e a técnica da detecção autohomodina, propomos um esquema de teletransporte bicolor usando um OPO.

Esse esquema experimental inédito será capaz de receber a informação de um dado estado quântico da luz em um comprimento de onda e, através do teletransporte, reconstruí-lo em um outro comprimento de onda, o que facilitará a conectividade entre sistemas físicos que operam em diferentes regiões do espectro.

A curto prazo, a ideia é implementar o protocolo e teletransportar estados coerentes somente como uma forma de prova de princípios, objetivo principal do presente doutorado. Entretanto, a ideia do nosso grupo é ambiciosa e vai além propondo, à médio prazo, a interação de feixes emaranhados produzidos em um OPO com um meio atômico formado por átomos frios de rubídio armadilhados, existente em nosso laboratório e que atualmente é usada em experimentos de EIT(Eletromagnetically Induced Transparency).

O objetivo final é transportar e distribuir a informação quântica contida nesses átomos armadilhados em uma faixa de frequência usada em sistemas de telecomunicações. Para isso, foi construído, paralelamente, no nosso laboratório um outro OPO triplamente ressonante trabalhando nas frequências das linhas $D_{1}$ e $D_{2}$ do rubídio e um outro na frequência da banda $\mathrm{C}^{1}$ com a finalidade de transmitir essa informação via fibras óticas.

\subsection{Visões gerais das propostas}

A proposta do teletransporte bicolor desenvolvida nesse doutorado passou por muitas mudanças de acordo com algumas limitações técnicas que tivemos ao longo do trabalho.

Inicialmente, visando um maior nível de emaranhamento entre os feixes gêmeos, optamos por construir um OPO duplamente ressonante, entretanto,

\footnotetext{
${ }^{1}$ Nome para a faixa de frequência utilizada em Telecom.
} 
o excesso de ruído de fase vindo do laser comercial e a alta potência requerida pelo OPO construído nos impediu de prosseguir com a montagem para o teletransporte. A alternativa que restou foi construir um OPO triplamente ressonante e aproveitar a informação quântica contida nos três feixes ressonantes envolvidos.

Para justificar a escolha inicial de um DROPO, assim como a alteração da montagem com um TROPO, apenas utilizaremos alguns resultados teóricos sem demonstrá-los.

Nessa seção, falaremos de forma geral sobre a evolução da proposta para o teletransporte bicolor e, no próximo capítulo, mostraremos as medidas que apresentaram excesso de ruído de fase e que nos levou a uma mudança completa no rumo do projeto.

\subsubsection{Proposta inicial com um DROPO}

Como já mencionado, o OPO é a fonte de estados emaranhados empregado no nosso sistema de teletransporte que tem sido intensamente investigado pelo nosso grupo em um regime de operação onde são produzidos dois feixes gêmeos intensos com polarizações ortogonais através do fenômeno conhecido como conversão paramétrica descendente.

Em essência, no modo de operação que utilizaremos no protocolo, esse dispositivo ótico é uma fonte de luz que converte a luz intensa incidente proveniente de um laser, no nosso caso, de $532 \mathrm{~nm}$ denominado feixe de bombeio, em dois feixes gêmeos de frequências mais baixas e comprimento de onda na região de 1064nm, denominados sinal e complementar, através da interação do feixe com um cristal $^{2}$ de suscetibilidade elétrica não-linear de segunda ordem.

O cristal por sua vez é inserido em uma cavidade ótica, travada em frequência, para intensificar o efeito da conversão paramétrica, selecionando os modos ressonantes na cavidade que será realimentado.

Os modos dos feixes gerados são refletidos pela cavidade e leva o cristal a um processo de emissão estimulada. Quando a taxa com que este processo ocorre (ou o ganho) é superior às perdas totais, produzem-se os feixes gêmeos intensos. A potência do bombeio na qual esse processo ocorre é denominada limiar de oscilação e é extremamente sensível às perdas seja dentro da cavidade, seja à perda do bombeio através do espelho de entrada [5, 14].

Dessa forma, a cavidade pode ser ressonante apenas para os feixes gêmeos convertidos, denominado DROPO (Double Resonant Optical Parametric Os-

\footnotetext{
${ }^{2} \mathrm{O}$ cristal utilizado nas nossas montagens é o potássio-titanil-fosfato, $\mathrm{KTiOPO}_{4}$, conhecido como KTP.
} 
cillator), ou ressonante para os três campos envolvidos (bombeio, sinal e complementar), denominado TROPO (Triply Resonant Optical Parametric Oscillator).

A Figura 6.5 exibe o esquema de montagem de um OPO em que um cristal não-linear, no interior de uma cavidade ótica, produz dois feixes nãodegenerados em frequência.

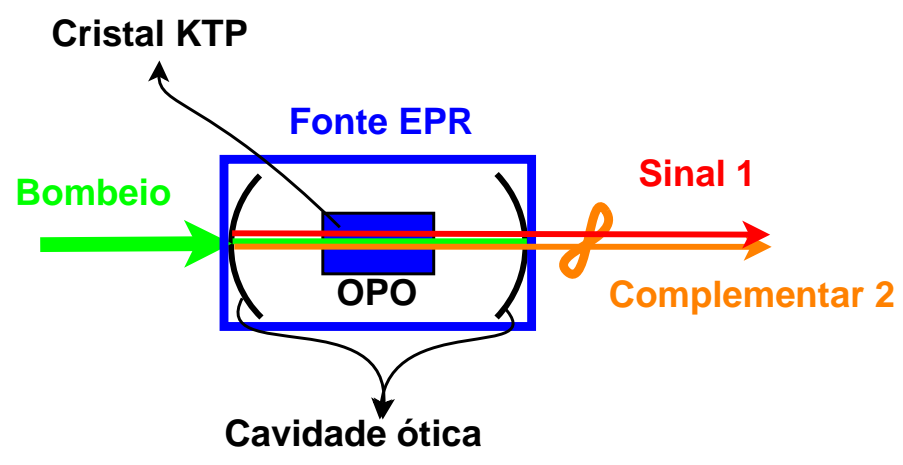

Figura 5.2: Esquema de um OPO. Cristal com suscetibilidade não-linear inserido em uma cavidade ótica e bombeado por um feixe intenso, no caso verde. São produzidos, nesse equema de oscilação, dois feixes gêmeos emaranhados que serve de fonte de estados EPR para o protocolo do teletransporte bicolor. Se a cavidade é ressonante somente para os gêmeos, o OPO é dito DROPO, e, se é ressonante para os três campos, é dito TROPO.

Em um trabalho teórico [58], foi demonstrado que a correlação das quadraturas dos feixes gêmeos no OPO seria o análogo ótico para o paradoxo EPR. Experimentalmente, esse resultado teórico foi confirmado pelo nosso grupo [1] pela primeira vez, garantindo que o OPO, operando em um modo onde feixes coerentes não-degenerados são produzidos, é uma fonte de estados quânticos emaranhados.

A demonstração experimental é feita, conforme abordaremos no próximo capítulo, através das medidas das quadraturas intensidade $(\hat{p})$ e fase $(\hat{q})$ dos gêmeos sinal (rotulado por 1 , cujas quadraturas são $\hat{p}_{1}$ e $\hat{q}_{1}$ ) e complementar (rotulado por 2, cujas quadraturas são $\hat{p}_{2}$ e $\hat{q}_{2}$ ).

A correlação intrinsecamente quântica entre observáveis, conhecida por emaranhamento, é verificada pela violação de algum critério de estados ditos separáveis. No caso do DROPO, o critério usado para a verificação do emaranhamento foi a violação da desigualdade de Duan et al. [59], conhecida como DGCZ.

Nesse critério, a variância da subtração das intensidades dos gêmeos, $\left\langle\Delta^{2} \hat{p}_{-}\right\rangle$, e a variância da soma das fases, $\left\langle\Delta^{2} \hat{q}_{+}\right\rangle$, dos mesmos obedece, para 
estados separáveis, a seguinte desigualdade:

$$
\left\langle\Delta^{2} \hat{p}_{-}\right\rangle+\left\langle\Delta^{2} \hat{q}_{+}\right\rangle \geq 2
$$

onde:

$$
\hat{p}_{-}=\frac{\left(\hat{p}_{1}-\hat{p}_{2}\right)}{\sqrt{2}}, \hat{q}_{+}=\frac{\left(\hat{q}_{1}+\hat{q}_{2}\right)}{\sqrt{2}} .
$$

A ideia inicial foi usar um DROPO porque o estado quântico dos feixes gêmeos produzidos, nesse tipo de OPO, são estados ditos puros. Grosseiramente falando, a pureza é uma grandeza que diz respeito ao quão próximo o estado do sistema está de um vetor de estado no espaço de Hilbert. Em um estado puro, sabe-se que a informação quântica é máxima, logo, o emaranhamento obtido é maior.

Além disso, como demonstrado anteriormente pelo nosso grupo [60], a interação entre os fótons e os fônons da rede cristalina provoca um aumento no ruído de fase que impede a verificação de emaranhamento dos gêmeos [60], ou seja, a violação da desigualdade (5.1). Para evitar a perda de emaranhamento, o cristal do esquema visto na Figura 6.5 é também resfriado na nossa montagem experimental, como será abordado no capítulo a seguir.

Optar por um OPO oscilando acima do limiar é a principal diferença entre a nossa proposta de teletransporte em variáveis contínuas e a do trabalho de Furusawa et al. em [7], que empregou um OPO oscilando abaixo do limiar.

Nesse modo de operação, o OPO produz feixes degenerados em estado de vácuo comprimido [61] que é um estado puro. Quando dois estados de vácuo comprimido são combinados em um BS, geram estados emaranhados.

Essa forma de gerar emaranhamento é a mais usada na literatura, entretanto ela apresenta uma limitação em relação à frequência dos feixes uma vez que elas devem ser iguais para poder interferir no BS. A Figura 5.3 ilustra a produção de emaranhamento mediante interferência de vácuos comprimidos gerados independentemente por um OPO operando abaixo do limiar.

Sabendo do potencial de um DROPO como fonte de emaranhamento de feixes em diferente frequências e dada a importância que um esquema de teletransporte bicolor teria para a comunicação quântica, projetamos um esquema para o protocolo usando um DROPO como fonte de estados emaranhados.

A Figura 5.4 exibe o esquema geral da montagem experimental imaginada. No próximo capítulo, veremos os resultados decisivos das medidas das quadraturas dos campos que não violaram (5.1) e que nos fizeram descartar a proposta com um DROPO.

Acompanhando a Figura, vemos que, logo após o OPO, há um cubo PBS usado para separar os feixes gêmeos de acordo com sua polarização. O feixe 


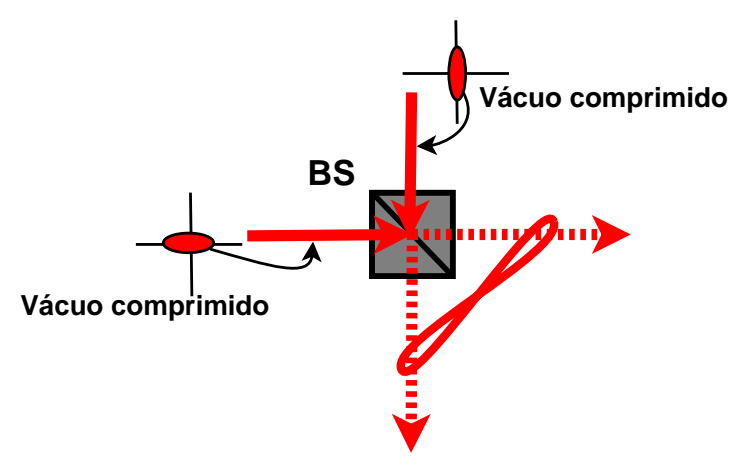

Figura 5.3: Esquema ilustrativo da geração de emaranhamento utilizado no teletransporte em [7]. Dois estados de vácuo comprimido, com uma diferença de fase entre eles, são gerados independentemente por um OPO oscilando abaixo do limiar. Esses estados são combinados em um BS produzindo estados emaranhados na saída.

sinal é utilizado por Victor, para construir o estado a ser teletransportado, e por Alice, na medida de Bell. Já o feixe complementar é entregue a Bob para que ele possa reconstruir o estado produzido por Victor.

O feixe gêmeo sinal é dirigido para uma cavidade ótica, chamada de filtro. Essa cavidade, ressonante em 1064nm, é travada na ressonância de modo que as bandas laterais, em outras frequências não-ressonantes na cavidade e onde encontra-se o emaranhamento, sejam refletidas.

A cavidade de filtro em $1064 \mathrm{~nm}$, quando travada na ressonância, permite a passagem de um feixe cujo estado quântico é coerente, uma espécie de campo clássico. Utilizando esse campo clássico, conforme será visto na próxima seção, Victor prepara o estado a ser teletransportado por Alice através de modulações feitas sobre o campo coerente com o uso de um EOM (modulador eletro-ótico).

Na estação operada por Alice, a medida de Bell é feita através da interferência, em um BS, das bandas laterais refletidas pela cavidade de filtro e do feixe modulado preparado por Victor. Alice utiliza uma cavidade ótica travada, chamada de análise, para medir a quadratura $\hat{q}$ do campo resultante da interferência no BS. As informações clássicas obtidas por Alice nesse processo são enviadas para Bob através de cabos elétricos e moduladores eletro-óticos. Ao receber as medidas clássicas de Alice, Bob as utiliza para modular a intensidade e fase do feixe gêmeo complementar recebido por ele. 


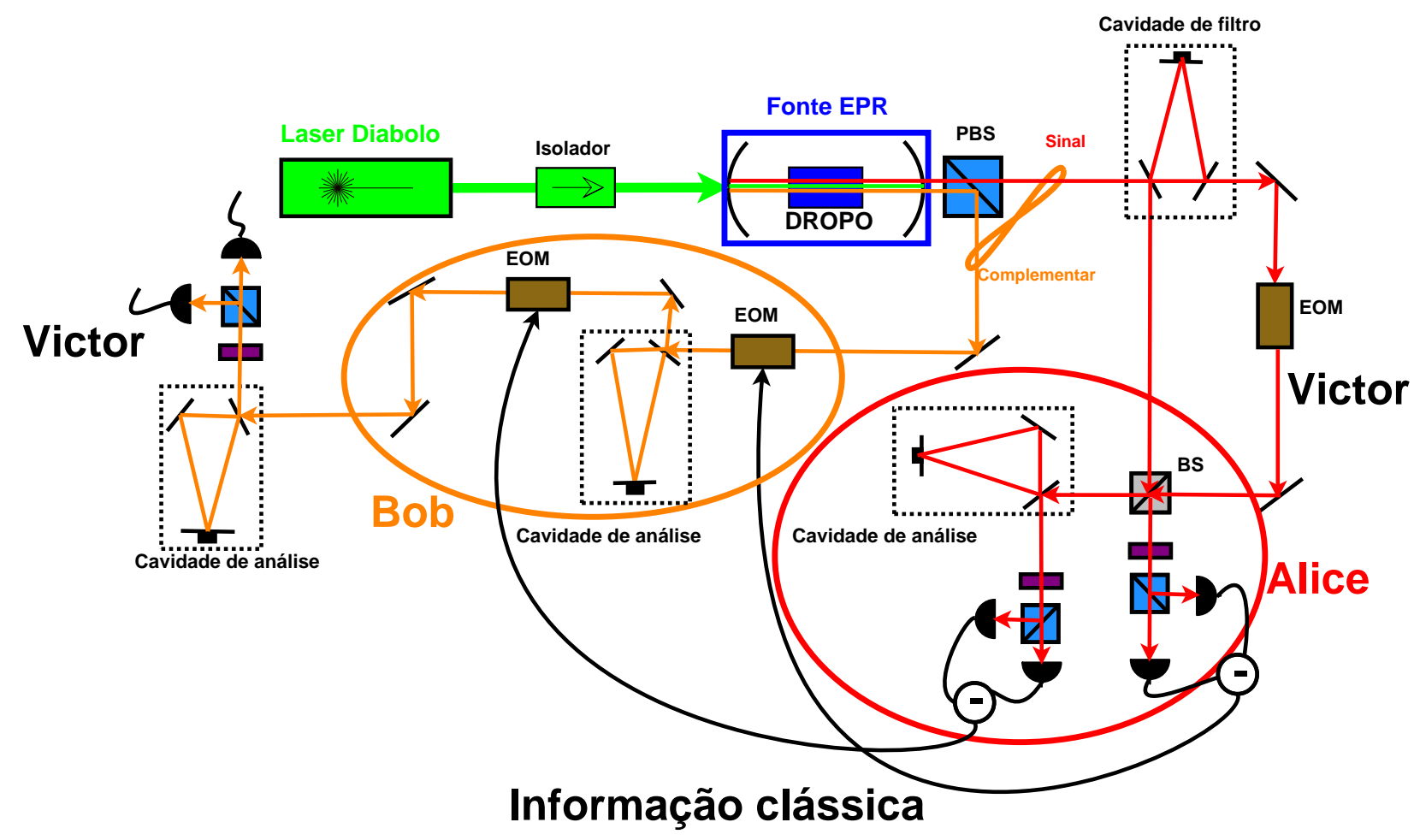

Figura 5.4: Esquema da proposta inicial do teletransporte quântico bicolor. Padrão de cores adotados para os elementos óticos: retângulo verde (isolador ótico), cubo cinza (beam splitter), cubo azul (PBS), retângulo roxo (lâmina de meia onda) e retângulo marrom (EOM). A fonte de estados emaranhados é um OPO duplamente ressonante que distribui os seus feixes emaranhados para Alice e Bob. O feixe de Alice passa, antes, por uma cavidade de filtro, travada em frequência, que transmite a portadora do campo e reflete as bandas laterais. A portadora é usada por Victor para preparar o estado a ser teletransportado e as bandas laterais são usadas por Alice para, junto com o estado preparado por Victor, realizar a "medida de Bell". Diferente do esquema de Furusawa et al em [7], Alice usa uma cavidade ótica para medir a fase e Bob também para reconstruir o estado. 


\subsubsection{Proposta atual com um TROPO}

As medidas das quadraturas dos feixes gêmeos do DROPO construído, descrito no capítulo a seguir, não apresentaram emaranhamento segundo o critério de DGCZ. Esse resultado nos levou a uma completa reformulação da proposta para o teletransporte bicolor que substituiu o DROPO, previamente montado, por um TROPO.

Demonstra-se que em um TROPO o estado quântico global, envolvendo os três feixes, é puro [62], o que significa que a informação quântica do sistema está contida nos três campos ressonantes na cavidade. Se considerarmos somente os gêmeos, por exemplo, perderemos a pureza do estado e, com isso, a informação contida no feixe refletido do bombeio.

Em um trabalho teórico do nosso grupo [1], demonstrou-se que um TROPO não-degenerado operando acima do limiar apresenta emaranhamento entre os três feixes ressonantes (bombeio, sinal e complementar). A confirmação experimental surgiu tempos depois [6] com o controle, através da diminuição da temperatura do cristal [60], do excesso de ruído de fase gerado da interação entre os fótons e os fônons da rede cristalina.

Como o sistema é dito tripartite, usa-se outro critério suficiente que envolve as três partições do sistema, como o critério visto em [63]. Nesse critério, os autores P. van Loock e A. Furusawa generalizaram, para $n$ partições de um sistema, o critério DGCZ (5.1), que se refere apenas as variâncias das combinações dos operadores $\hat{p}$ e $\hat{q}$ das duas partições do sistema $\left(\hat{p}_{1}, \hat{q}_{1} ; \hat{p}_{2}, \hat{q}_{2}\right)$.

Considerando-se as quadraturas das três partições (bombeio: $\hat{p}_{0}, \hat{q}_{0}$; sinal: $\hat{p}_{1}$, $\hat{q}_{1}$ e complementar: $\hat{p}_{0}, \hat{q}_{0}$ ), o critério [63] de inseparabilidade tripartite implica na violação de pelo menos duas das três desigualdades [6], ou seja, a violação de duas inequações significa o emaranhamento de dois pares de feixes:

$$
\begin{aligned}
& \left\langle\Delta^{2}\left(\frac{\hat{p}_{1}-\hat{p}_{2}}{\sqrt{2}}\right)\right\rangle+\left\langle\Delta^{2}\left(\frac{\hat{q}_{1}+\hat{q}_{2}-\alpha_{0} \hat{q}_{0}}{\sqrt{2}}\right)\right\rangle \geq 2 \\
& \left\langle\Delta^{2}\left(\frac{\hat{p}_{0}+\hat{p}_{2}}{\sqrt{2}}\right)\right\rangle+\left\langle\Delta^{2}\left(\frac{\alpha_{1} \hat{q}_{1}+\hat{q}_{2}-\hat{q}_{0}}{\sqrt{2}}\right)\right\rangle \geq 2 \\
& \left\langle\Delta^{2}\left(\frac{\hat{p}_{0}+\hat{p}_{1}}{\sqrt{2}}\right)\right\rangle+\left\langle\Delta^{2}\left(\frac{\hat{q}_{1}+\alpha_{2} \hat{q}_{2}-\hat{q}_{0}}{\sqrt{2}}\right)\right\rangle \geq 2
\end{aligned}
$$

Como estamos apenas interessados na correlação entre os gêmeos para a realização do teletransporte bicolor, consideremos apenas a inequeção (5.3).

Ela pode ser encarada como a desigualdade de DGCZ com um termo a mais devido à correlação entre o bombeio e a combinação dos feixes gêmeos. O parâmetro $\alpha_{0}$ determina o peso dessa correlação e podemos minimizá-lo a 
fim de achar o valor a partir do qual é possível o máximo de emaranhamento dos gêmeos.

Para isso, lembrando-se a definição de variância de operadores, basta minimizar o segundo termo da inequação (5.3):

$$
\frac{d\left\langle\left(\hat{q}_{1}^{2}+\hat{q}_{2}^{2}+\alpha_{0}^{2} \hat{q}_{0}^{2}+2 \alpha_{0} \hat{q}_{0} \hat{q}_{1}+2 \alpha_{0} \hat{q}_{0} \hat{q}_{1}+2 \alpha_{0} \hat{q}_{2} \hat{q}_{2}\right)\right\rangle}{d \alpha_{0}}=0
$$

Concluimos que o valor mínimo é:

$$
\alpha_{0}=\frac{\left\langle\hat{q}_{0}\left(\hat{q}_{1}+\hat{q}_{2}\right)\right\rangle}{\left\langle\Delta^{2} \hat{q}_{0}\right\rangle}
$$

Dessa forma, com um TROPO como fonte de emaranhamento, é necessário incluir a informação de fase do bombeio, $\hat{q}_{0}$, para que a inequação (5.3) seja maximamente violada.

Na prática, no esquema do teletransporte bicolor, isso é feito somando essa informação de fase do bombeio à informação de fase medida por Alice, antes de ser enviada para Bob.

Assim, foi preciso fazer algumas alterações no projeto da proposta inicial e acrescentar mais duas cavidades óticas, conforme é exibido na Figura 5.5. Essa Figura mostra o esquema atual do teletransporte bicolor que estamos implementando, basicamente o mesmo esquema da Figura 5.4, porém com a presença de um TROPO e de mais duas cavidades óticas para o 532nm: uma de filtro e outra de análise.

Para filtrar o excesso de ruído de fase vindo do laser Diabolo, usamos uma cavidade de filtro, ressonante em 532nm. A montagem da cavidade, assim como a técnica utilizada para o travamento é abordada no próximo capítulo.

Além da cavidade de filtro, é observada outra cavidade ótica para o feixe $532 \mathrm{~nm}$ refletido do TROPO que tem a função de recuperar parte da informação quântica do estado global contida no ruído de fase do bombeio, $\hat{q}_{0}$. Ela será travada de forma que, durante a realização da medida, usando-se a técnica da detecção auto-homodina, somente seja possível medir a informação de fase desse feixe. 


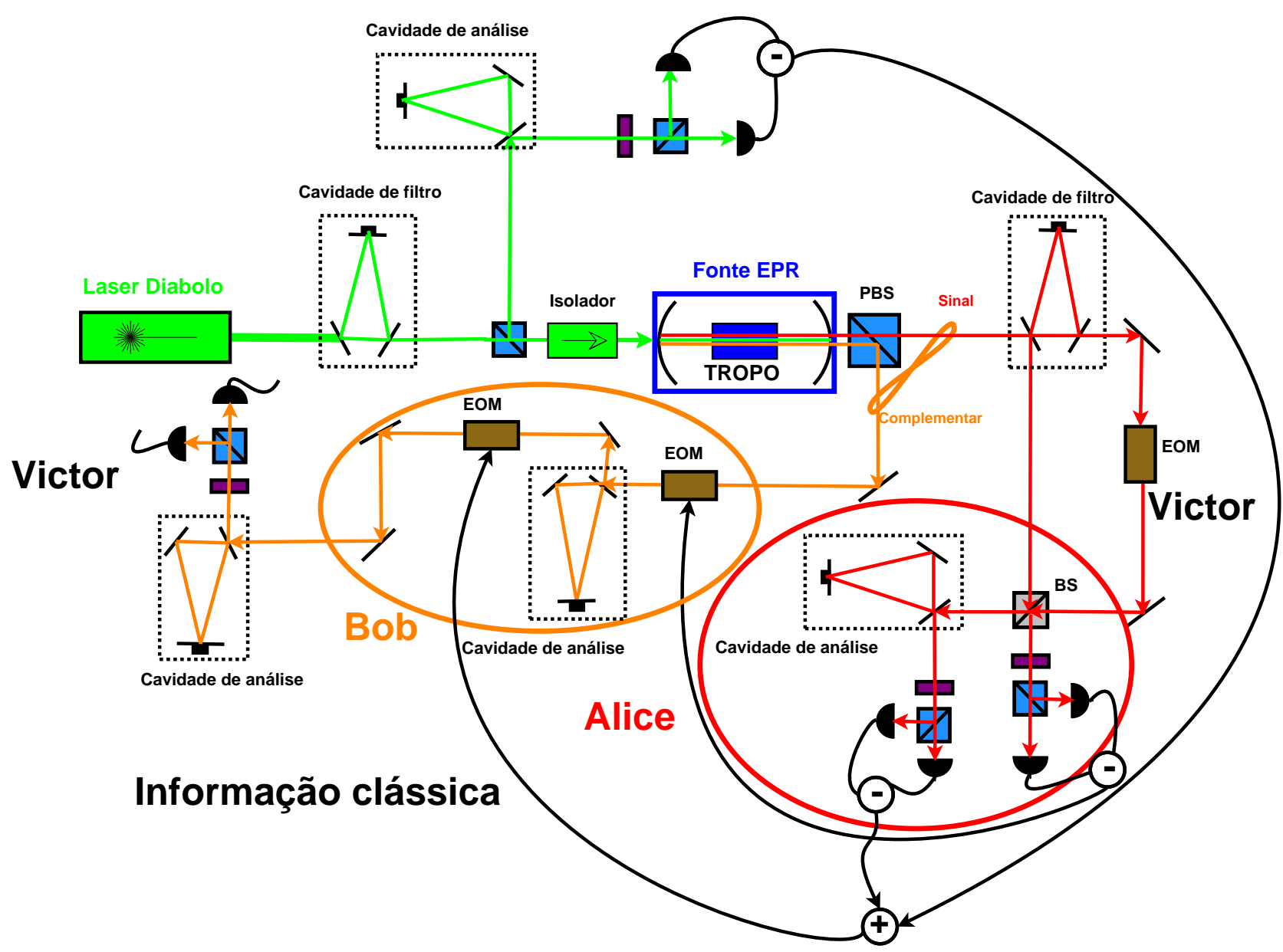

Figura 5.5: Esquema atual da proposta para o teletransporte bicolor. Padrão de cores adotados para os elementos óticos: retângulo verde (isolador ótico), cubo cinza (beam splitter), cubo azul (PBS), retângulo roxo (lâmina de meia onda) e retângulo marrom (EOM). Para filtrar o ruído de fase do laser, foi acrescentada uma cavidade de filtro que é travada em frequência durante o experimento. A fonte de estados emaranhados é um OPO triplamente ressonante e, por conta disso, acrescentou-se mais uma cavidade ótica para a reflexão do verde, que é travada de forma a extrair a informação da fase desse feixe refletido, $\hat{q_{0}}$. A principal diferença entre a proposta da Figura 5.4 e esta é a forma como Alice extrai informação de fase dos campos. Nesta nova proposta, por conta do feixe verde refletido, é necessário somar a informação de fase contida nesse feixe de $532 \mathrm{~nm}$ com o resto da informação de fase medida por Alice do feixe em 1064nm. 


\section{Capítulo 6}

\section{Montagem experimental e resultados preliminares}

Esse capítulo versa sobre a tentativa de implementação experimental da nossa proposta para o teletransporte bicolor descrita no capítulo anterior. Abordaremos pontos fundamentais da montagem como a caracterização dos detectores e a calibração do "shot noise"; a construção da nova câmara de vácuo que abriga a fonte de estados emaranhados e o projeto e construção das novas cavidades de análise usadas na detecção auto-homodina dos feixes.

Além disso, falaremos sobre o canal clássico utilizado e a caracterização desses dispositivos. E, finalmente, abordaremos sobre os projetos dos candidatos a fonte de estados emaranhados do protocolo: DROPO1, DROPO2, TROPO1, TROPO2. Focaremos, nesse ponto, na montagem desses OPOs e, no caso do DROPO2 e TROPO2, na caracterização do nível de ruído quântico de intensidade e fase. Apesar de ter atingindo um nível de compressão em intensidade com o DROPO2 nunca antes medido no $L M C A L$, deparamos com um excesso de ruído de fase que degradou o emaranhamento dos feixes e impediu o prosseguimento da montagem proposta para o teletransporte bicolor com essa fonte. Atualmente, estamos investindo no TROPO2 como provável canal quântico do protocolo e os primeiros resultados quânticos são mostrados.

Abordaremos também o nosso esforço em neutralizar outra fonte de ruído externo, dessa vez, de origem mecânica que dificultou o travamento das cavidades óticas. Tal esforço implica na construção de estruturas mecânicas, que servem de suportes de espelhos, cada vez mais robustas. 


\subsection{Caracterização dos detectores}

Vimos que o "shot noise" é a escala de medida de ruído quântico, ou seja, todo ruído quântico medido será comparado a essa referência de medida. Assim, ao iniciar qualquer medida em Ótica Quântica é preciso antes estabelecer ou calibrar essa referência, de forma a verificar o perfeito funcionamento da eletrônica do tipo LIT envolvida.

Nessa seção, será descrita como é feita experimentalmente a calibração do "shot noise", abordada teoricamente no capítulo 3. Discutiremos também a eficiência quântica medida dos fotodiodos ETX300 e a saturação dos detectores, além da questão do ruído eletrônico e como ele é descontado das medidas. Por fim, exemplificaremos como a presença de circuitos eletrônicos que não são do tipo LIT distorcem a medida do ruído quântico padrão.

Em todas as nossas montagens experimentais usamos o laser comercial Diabolo da marca alemã Innolight $G m b H$ do tipo $N d: Y A G$ que produz um feixe principal em $532 \mathrm{~nm}$, atualmente, com $700 \mathrm{~mW}$ de potência e um BW de $1 K H z$. Além desse feixe, o Diabolo tem outra saída para um feixe $I R$ com potência de $250 \mathrm{~mW}$ que utilizamos na caracterização e calibração de certos dispositivos e alinhamento do OPO.

\subsubsection{Eficiência medida e saturação do detector}

De acordo com a discussão anterior, os fotodiodos ETX300 empregados na montagem possuem uma responsividade de $0,78 \mathrm{~A} / \mathrm{W}$, segundo seu fabricante Epitaxx, o que resulta em uma eficiência quântica de $90 \%$. Como o sistema de medição de ruído quântico através das fotocorrentes é muito sensível às perdas, é preciso verificar esse valor fornecido pelo fabricante.

Além disso, como o sistema eletrônico tem ser do tipo LIT, é necessário também descobrir qual o limite da potência da luz incidente que satura o detector, ou seja, qual a potência a partir da qual o sinal DC do detector não varia mais.

Sabemos, da seção 3.1, que a razão entre a fotocorrente gerada na detecção, $i_{D C}$, e a potência da luz incidente ${ }^{1}, P(t)$, é igual a $0,86 \eta$. Porém, devido a amplificação de transimpedância que ocorre no circuito do detector, a medida realizada é uma medida de tensão elétrica com um certo fator de ganho, que denotaremos por $G_{D C}$. Assim, temos que a tensão DC $\left(V_{D C}\right)$ medida de um detector, observando-se a Figura 3.6, é igual a:

$$
V_{D C}=i_{D C}\left(R_{1}+R_{2}\right) G_{D C}
$$

\footnotetext{
${ }^{1}$ no caso do comprimento de onda igual a $1064 \mathrm{~nm}$
} 
o que resulta em uma razão igual a:

$$
\frac{V_{D C}}{P(t)}=0,86 \eta G_{D C}\left(R_{1}+R_{2}\right)
$$

Então, para verificar experimentalmente a eficiência quântica, $\eta$, do fotodiodo basta medir a tensão $V_{D C}$ do detector em função da potência de luz incidente $P(t)$ e determinar o coeficiente angular da reta obtida, denotado por $K$. Vamos ilustrar uma medida desse tipo que realizamos com um dos detectores da montagem atual, só que para os seguintes valores: $G_{D C}=1$ e $R_{1}+R_{2}=$ $1 K \Omega$. Nesse caso específico, a eficiência é simplesmente:

$$
\eta=\frac{K}{860}
$$

Essas medidas foram realizadas através da mesma montagem experimental utilizada na calibração do "shot noise" para os detectores, vista na Figura 6.1. Com uma lâmina de meia onda e um cubo PBS, varia-se a intensidade de luz incidente nos detectores e, com um power meter ${ }^{2}$, mede-se o valor dessa potência. O gráfico da Figura 6.2 ilustra a depedência linear entre essas grandezas físicas medidas e o ajuste linear obtido para um dos detectores, que fornece uma eficiência de 91(1)\%.

Um ponto importante a ressaltar é sobre a questão da mudança do limite de saturação do detector. Para feixes gêmeos com potências maiores que $20 \mathrm{~mW}$, esse detector saturaria e deixaria de ser do tipo LIT. Assim como aconteceu com a montagem do DROPO2, descrito na seção 6.5, tivemos que alterar esse limite mudando o valor da série de resistências para $490 \Omega$. Segundo a equação (6.2), vemos que a potência de saturação, nesse caso, praticamente dobra.

Ao longo do trabalho, é muito comum alterar os valores dos componentes (vistos nas Figuras 3.6 e B.1) $C_{1}, R_{1}, C_{3}$ e $R_{4}$ do circuito do detector de forma a alterar as frequências de corte dos filtros e o limite de saturação do detector. Os valores dos componentes referente as medidas de caracterização DC e HF apresentadas como exemplo nesta seção são: $C_{1}=22 \mathrm{nF}, R_{1}=900 \Omega$, $C_{3}=100 \mathrm{nF}$ e $R_{4}=1 K \Omega$.

\subsection{2 "Shot noise" e o ruído eletrônico}

A fim de avaliar possíveis não-linearidades e perdas, que comprometem a medida de ruído quântico, o primeiro passo em uma montagem eletrônica de um experimento de Ótica Quântica é calibrar o sistema de medição (toda a

\footnotetext{
${ }^{2}$ Optical Power and Energy meter (PM100D) da Thorlabs.
} 


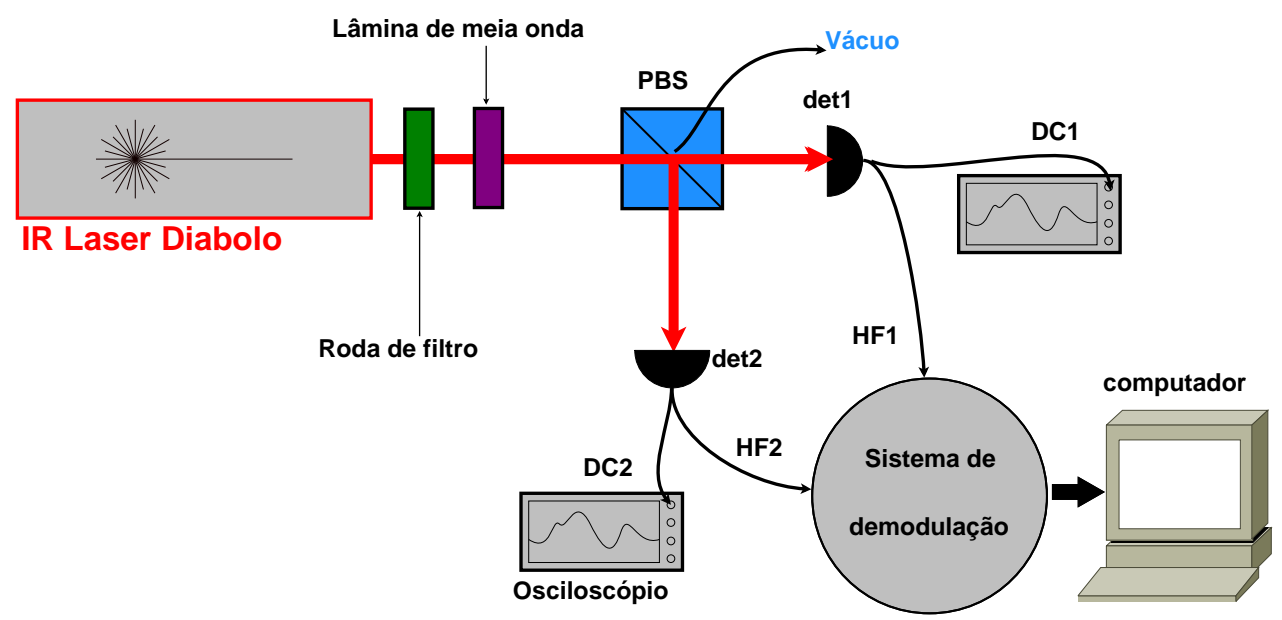

Figura 6.1: Montagem para a caracterização dos detectores do experimento usando a saída IR do Laser Diabolo. As medidas para caracterizar a eficiência dos fotodiodos foram feitas usando-se um osciloscópio e um medidor de potência. E as medidas da calibração do "shot noise" foram realizadas pelo sistema de medida, que compreende filtragens, amplificações, demodulação e a aquisição das medidas via computador.

cadeia de amplificações, filtragens e demodulação) utilizando os detectores e o laser.

A caracterização dos detectores foi feita para poder testar se a eletrônica dos mesmos respondia apropriadamente de acordo com a teoria sobre sistemas LIT, vista na seção A. Através da montagem da Figura 6.1, estudamos o comportamento quântico dos quatros detectores envolvidos na montagem do teletransporte.

Para verificar se um detector está em condições de servir em uma medida quântica, faz-se necessário verificar o seu comportamento durante a homodinagem com o vácuo, ou seja, calibrá-lo usando o estado com as menores flutuações quânticas, o vácuo.

Usamos, para isso, a saída IR do Diabolo como oscilador local. Com a roda de filtro, altera-se a potência da luz que incide na lâmina de meia onda. Com a lâmina e o PBS, balanceia-se a potência de luz que incide nos detetores det1 e det2. A ideia de empregar no experimento uma lâmina + um PBS, ao invés de um BS 50 : 50 para dividir o feixe incidente, é ter maior controle fino da divisão de potência do feixe entre os detectores.

Segundo a teoria, tanto a variância da subtração das fotocorrentes quanto a variância da soma, no caso da homodinagem com o vácuo, são linearmente dependentes da potência $\left(\beta^{2}\right)$ da luz incidente na lâmina da Figura 6.1. 


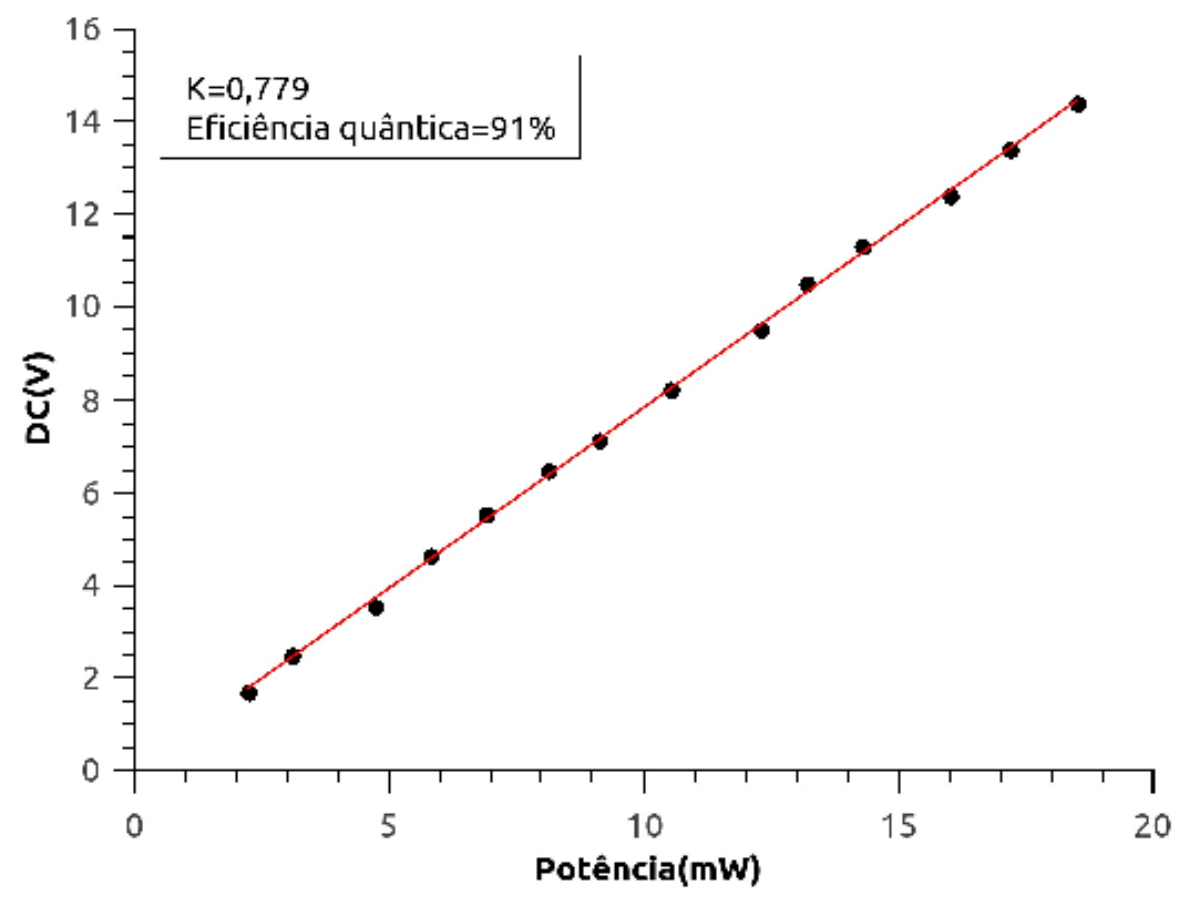

Figura 6.2: Resultado das medidas de $V_{D C}$ em função de $P(t)$ de um dos detectores da montagem para os valores: $G_{D C}=1$ e $R_{1}+R_{2}=1 K \Omega$. O ajuste linear da reta obtida forneceu um valor de $K$ igual a $0,779 \mathrm{~V} / \mathrm{mW}$, o que, segundo a equação (6.3), implica em uma eficiência quântica de aproximadamente $91(1) \%$. Foi verificado, para esses valores de resistência e ganho, que a saturação do detector ocorre em torno de $20 \mathrm{~mW}$ de potência. 
Experimentalmente, a informação quântica das fotocorrentes detectadas encontra-se na saída HF dos detectores que é adquirido via computador através da demodulação destes sinais, denotados ${ }^{3}$ por $H F 1_{D C}^{\text {shot medido }}$ e $H F 2_{D C}^{\text {shot medido }}$.

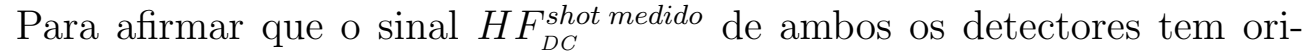
gem somente no caráter quântico da luz detectada, é necessário descontar a contribuição do ruído eletrônico ao sinal. Como dito previamente, o ruído eletrônico tem origem no ruído térmico, no "dark noise" e em outros ruídos de componentes eletrônicos.

O sinal denotado por $H F_{D C}^{\text {shot }}$, originado somente da luz, é igual a $H F_{D C}^{\text {shot medido }}$ menos o sinal devido ao ruído eletrônico, chamado de $H F_{D C}^{\text {eletro }}$. Como os si-

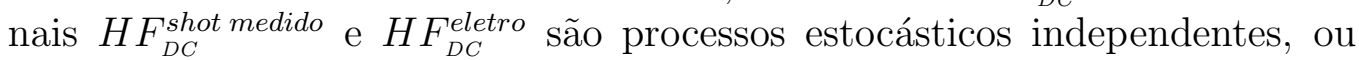
não-correlacionados, então:

$$
\overline{\left(H F_{D C}^{\text {shot medido }}\right)\left(H F_{D C}^{\text {eletro }}\right)}=\left(\overline { H F _ { D C } ^ { \text { shot medido } } ) } \left(\overline{\left.H F_{D C}^{\text {eletro }}\right)}\right.\right.
$$

Isso implica que a variância $H F_{D C}^{\text {shot }}$ é:

$$
\operatorname{Var}\left(H F_{D C}^{\text {shot }}\right)=\operatorname{Var}\left(H F_{D C}^{\text {shot medido }}\right)-\operatorname{Var}\left(H F_{D C}^{\text {eletro }}\right)
$$

A medida da potência do feixe incidente é feita indiretamente por meio da tensão $V_{D C}$ (ou saída DC do detector), conforme a equação (6.2). Para verificar, na prática, o perfeito funcionamento da eletrônica de todo o sistema de detecção, a relação entre a variância da subtração dos sinais demodulados dos detectores e a tensão DC medida (a mesma para os dois detectores, por hipótese) deve ser, claramente, linear dependendo de um fator de ganho, $k_{\text {eletro }}$, instrisecamente relacionado à toda eletrônica envolvida nos processamentos dos sinais DC e HF:

$$
\operatorname{Var}\left(H F 1_{D C}^{\text {shot }}-H F 2_{D C}^{\text {shot }}\right)=k_{\text {eletro }} V_{D C}
$$

A Figura 6.3 exibe, em caráter ilustrativo, os processos estocásticos dos sinais $H F_{D C}^{\text {eletro }}$ e dos ruídos quânticos padrões $\left(H F_{D C}^{\text {shot medido }}\right)$ referentes a incidência de luz para duas potências diferentes. Os processos tem 1.000 pontos e foram adquiridos em uma janela temporal de $1.6 \mathrm{~ms}$ e estão na mesma escala. Como é esperado, o nível de ruído eletrônico é muito menor que o nível de ruído devido à luz incidente, evidenciando a importância de empregar somente componentes eletrônicos que produzem baixo ruído eletrônico.

\footnotetext{
${ }^{3}$ Essa notação é só para lembrar que a homodinagem é realizada com o vácuo e a informação quântica medida vem dos sinais HF dos detectores que, ao passar pela demodulação, transforma-se em sinais do tipo DC.
} 
Experimentos em Ótica Quântica como o nosso só foram possíveis graças ao desenvolvimento e aprimoramento de componentes tais como fotodiodos, amplificadores operacionais, diodos e resistores de baixo ruído que não "escondem" a informação quântica proveniente da luz detectada.

A Figura 6.4 mostra os gráficos resultantes de 10.000 medidas, realizadas em $15 \mathrm{MHz}$, para a calibração do "shot noise" descontadas do ruído eletrônico. Observa-se que a equação (6.6) foi verificada para $k_{\text {eletro }}=0,0117(5)$.

Ainda na mesma figura, é possível constatar que o comportamento de $\operatorname{Var}\left(H F 1_{D C}^{\text {shot }}+H F 2_{D C}^{\text {shot }}\right)$ deixa de ser linear a partir de um certo valor de $D C$. A razão desse comportamento não esperado, para a variância da soma dos sinais é que o estado quântico da luz produzido pelo laser comercial, na prática, é um feixe com excesso de ruído.

Por conta dessa "imperfeição" do oscilador local, a calibração de toda medida de ruído quântico da luz é feita através da variância da subtração dos sinais $\left(\operatorname{Var}\left(H F 1_{D C}^{\text {shot }}-H F 2_{D C}^{\text {shot }}\right)\right)$ ou pela constante $k_{\text {eletro }}$.

Se a homodinagem ${ }^{4}$ for realizada com luz em um estado quântico comprimido, por exemplo, a quantificação (medida linear, denotada por Med) do ruído gerado pela luz neste estado é dado, em uma escala linear, por:

$$
\operatorname{Med}(\text { linear })=\frac{\operatorname{Var}\left(H F 1_{D C} \pm H F 2_{D C}\right)}{\operatorname{Var}\left(H F 1_{D C}^{\text {shot }}-H F 2_{D C}^{\text {shot }}\right)}=\frac{\operatorname{Var}\left(H F 1_{D C} \pm H F 2_{D C}\right)}{k_{\text {eletro }}}
$$

A medida (Med) do nível de ruído também pode ser expressa em escala logarítimica, de maneira que, definido pela equação (3.45), é equivalente a:

$$
\operatorname{Med}(d B)=10 \log \left(\frac{\operatorname{Var}\left(H F 1_{D C} \pm H F 2_{D C}\right)}{\operatorname{Var}\left(H F 1_{D C}^{\text {shot }}-H F 2_{D C}^{\text {shot }}\right)}\right)
$$

Uma medida negativa do nível de ruído da soma ou subtração dos sinais $H F_{D C}$ das fotocorrentes implica em uma compressão de ruído quântico. No caso de uma medida positiva, implica em excesso de ruído quântico com relação ao nível de ruído quântico do "shot noise". Por essa razão, o "shot noise" é conhecido como ruído quântico padrão ou limite quântico padrão (Standard Quantum Limit- SQL).

\subsection{Sistema de refrigeração e montagem}

Para implementar o esquema do teletransporte bicolor, é necessário controlar todas as fontes de ruído que não tenham origem na natureza quântica dos

\footnotetext{
${ }^{4}$ No nosso experimento, empregamos a auto-homodinagem como método de medida das quadraturas da luz.
} 

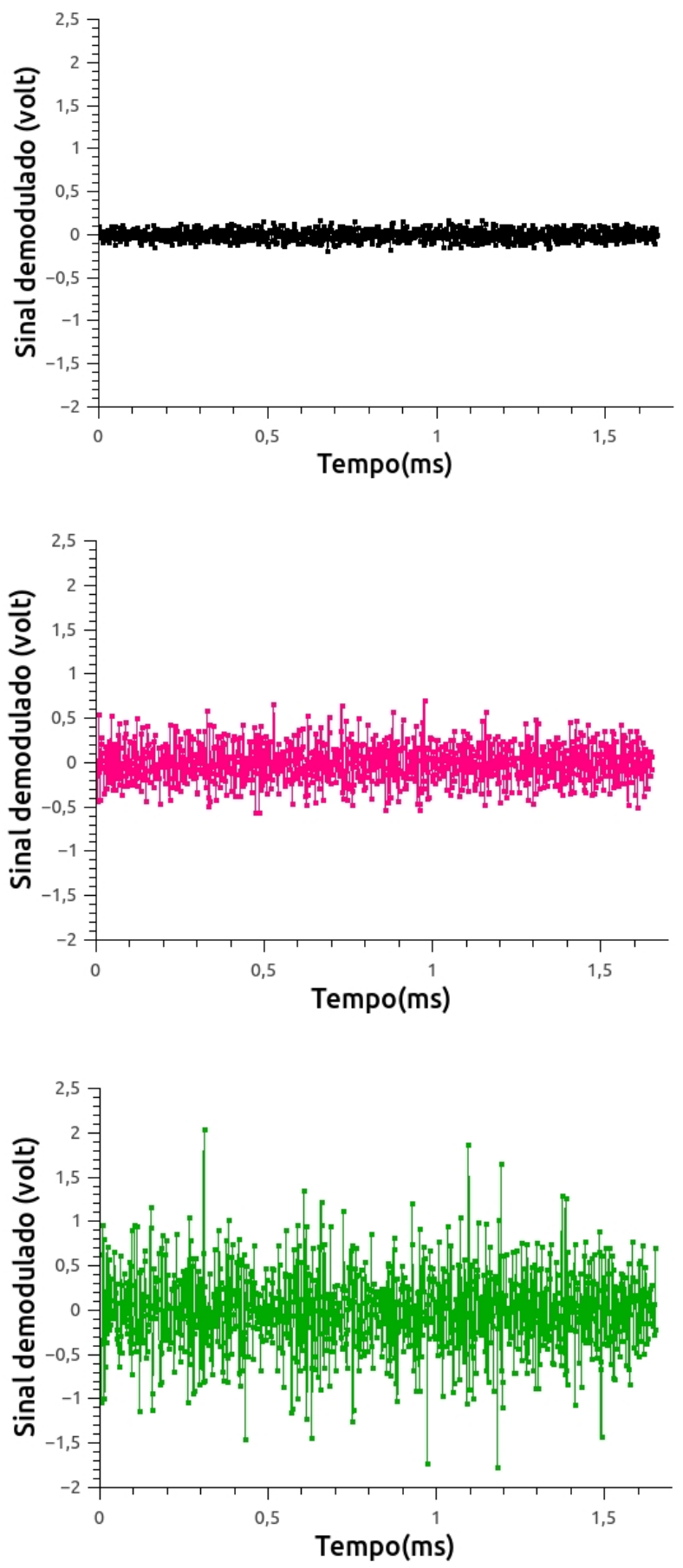

Figura 6.3: Figuras meramente ilustrativas dos processos estocásticos resultantes da demodulação do sinal $\mathrm{HF}$ de um detector e adquirido via computador em uma janela temporal de 1,6ms. Em preto, representa o sinal demodulado sem a incidência de luz, denomidado de ruído eletrônico. Em rosa, o sinal do mesmo detector só que com luz incidindo com uma potência de $6.3 \mathrm{~mW}$ ( $5 \mathrm{~V}$ de $\mathrm{DC}$ ) e, em verde, com uma potência de $18 \mathrm{~mW}(14 \mathrm{~V}$ de DC). Todos os sinais foram obtidos para uma frequência de $15 \mathrm{MHz}$. 

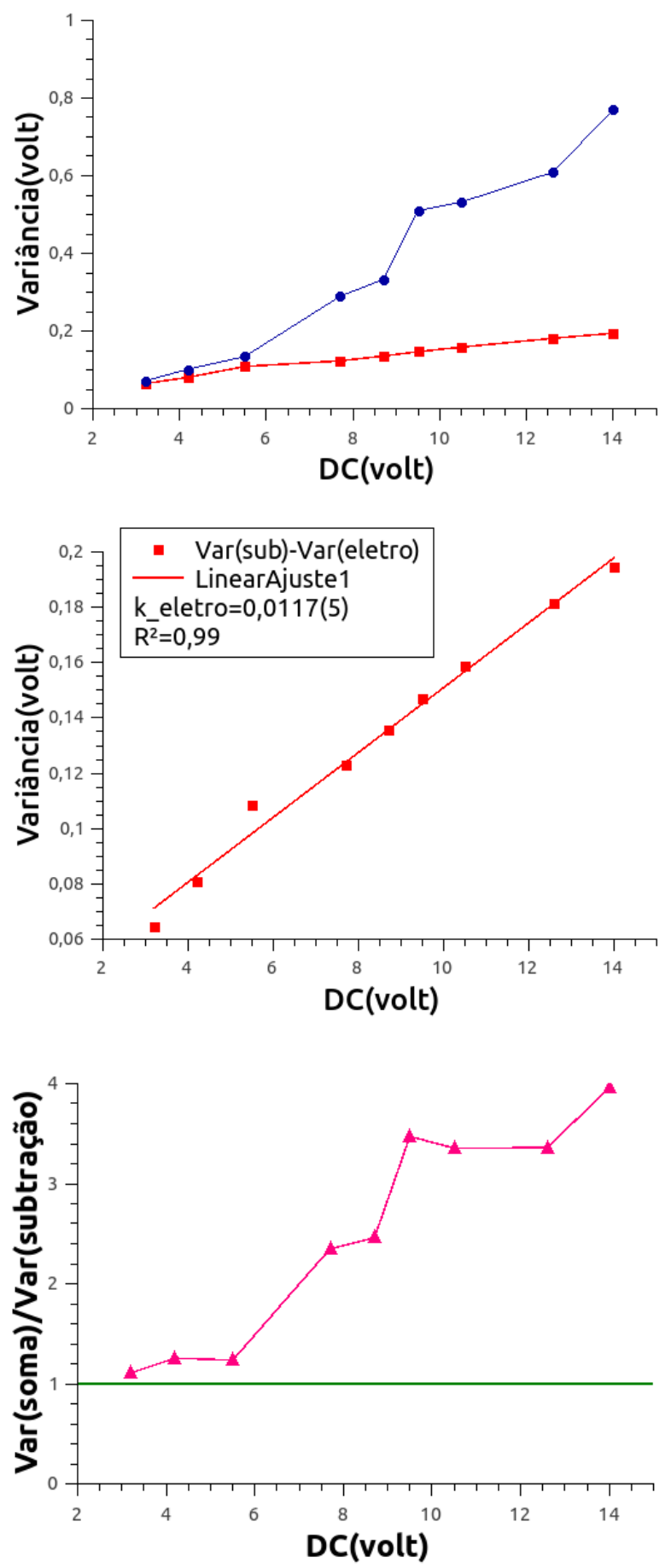

Figura 6.4: Resultado das medidas de calibração do "shot noise" para um dos detectores da montagem vista na Figura 6.1. No primeiro gráfico, é ilustrado o comportamento das variâncias da soma (em azul) e da subtração (em vermelho) das fotocorrentes geradas. No segundo, é visto o comportamento esperado para variância da subtração que varia linearmente com o sinal DC do detector, ou em outras palavras, com a potência da luz incidente. $\mathrm{Na}$ última figura, é vista a razão entre estas variâncias em função do sinal DC. Medidas realizadas em $15 \mathrm{MHz}$. 
feixes detectados. Isso porque esse tipo de ruído degrada o nível de ruído quântico dos feixes emaranhados e, consequentemente, afeta a qualidade, ou fidelidade, do protocolo.

É de conhecimento do nosso grupo que uma dessas fontes de ruído, que provoca um excesso de ruído de fase nos feixes gêmeos e prejudica o emranhamento, diz respeito a interação entre os fótons do bombeio e os fônons [60] da rede cristalina do material não-linear, $K T P$, usado para produzir os gêmeos. Experimentalmente, esse problema é contornado diminu- indo-se a temperatura do cristal KTP, o que reduz apreciavelmente o excesso de ruído de fase. Entretanto, ao resfriar o cristal, há a condensação de água do ambiente na sua superfície, impedindo a criação dos feixes sinal e complementar.

Assim, antes de começar o alinhamento da fonte de estados emaranhados propriamente, que será descrita na seção 6.5, foi preciso projetar e construir um novo esquema de refrigeração do cristal que incluiu uma nova câmara de vácuo e uma estrutura de cobre, no qual circula água a temperatura ambiente. A água é usada para reduzir a temperatura da fonte quente do dispositivo elétrico, chamado de Peltier, empregado para refriar o cristal.

Tanto o projeto quanto a construção desse sistema de refrigeração foram realizado nas oficinas mecânicas do IFUSP e contou com a participação e trabalho de alguns técnicos do instituto das áreas de desenho técnico, mecânica, vácuo e soldagem.

\subsubsection{Câmara de vácuo}

A construção da nova câmara de vácuo que abriga o OPO procurou atender todas as nossas necessidades práticas para a execução do experimento tais como: construir flanges que pudessem abrigar um visor (ou janela ótica antirefletora) para a passagem da luz; construir outras flanges que permitissem a passagem adequada da fiação para dentro da câmara e a construção de uma base que fosse possível fixar os suportes de espelhos e lentes. A idéia foi fazer uma câmara de vácuo que atendesse os padrões da indústria de equipamentos para vácuo e que utilizasse uma bomba mecânica de sucção, um tipo de vácuo considerado simples. Para nossos propósitos de evitar a condensação da umidade do ambiente em torno do cristal, um vácuo da ordem de $10^{-1} \mathrm{mBar}$ já é o suficiente.

Todo o corpo e tampa da câmara, assim como todos os seus "pescoços" e suas flanges, foram feitos em inox e, somente a sua base foi contruída em alumínio. Os "pescoços" e as flanges contruídas seguiram o padrão internacional NW16 e NW50 para facilitar na compra das abraçadeiras de alumínios, que fixam as flanges aos "pescoços", e dos o-rings que vedam a passagem de ar entre as flanges e os "pescoços" da câmara. A Figura 6.6 mostra duas 
fotografias do corpo da câmara e de sua base prontas.

A câmara possui 8 "pescoços", dos quais 2 são do tipo $N W 16$ e os restantes do tipo $N W 50$. Através dos $N W 16$ são conectados a saída da bomba mecânica e o sensor de pressão usado para monitorar a pressão no interior da câmara. Os do tipo NW50 são usados para fixar as duas flanges que abrigam as janelas óticas(vista na Figura 6.8), por onde entra e sai a luz na câmara e para fixar um tipo especial de flange, o feedthough ${ }^{5}$, onde passa toda a fiação elétrica para dentro da câmara. Além disso, esse tipo NW50 também é utilizado para fixar um outro tipo especial de flange, construída nas oficinas do IFUSP, que permite a passagem de água, usada para resfriar a fonte quente do Peltier, para dentro da câmara. A água circula dentro da câmara devido à uma bomba ${ }^{6}$ que permanece ligada durante toda a realização do experimento.

Antes da montagem na mesa ótica do LMCAL, a câmara de vácuo passou por um teste de vácuo para verificar se havia fontes de vazamento. O teste consiste basicamente em bombear continuamente a câmara e usar um gás, no caso usou-se o gás Hélio, para detectar potenciais fontes de vazamento. Para isso,injeta-se, com auxílio de uma ponta de prova, o gás nas regiões suspeitas de existir vazamento(por exemplo, nas soldas). Caso tenha vazamento, o gás penetra na câmara e logo em seguida é bombeado, passando por um detector sensível ao gás Hélio, onde sua concentração é medida. O vácuo obtido com esse teste foi da ordem de $10^{-6} \mathrm{mBar}$. Na Figura 6.5, é vista a realização do teste de vácuo. Após o teste de vácuo, fixou-se a câmara à sua base. A Figura 6.7 exibe uma visão geral da câmara de vácuo em operação no laboratório. O corpo da câmara é preso à sua base de alumínio através de parafusos não-passantes e a base, por sua vez, é fixada à mesa ótica por meio de parafusos passantes. A base de alumínio tem o mesmo padrão de furação da mesa ótica para poder fixar tanto o suporte que sustenta o cristal quanto os suportes dos espelhos.

\subsubsection{Montagem no interior da câmara}

Para descrever a montagem mecânica da nossa fonte de estados emaranhados, OPO, situada dentro da câmara de vácuo, vamos primeiro começar pela montagem do cristal KTP.

Como dito anteriormente, o cristal é o elemento ótico a ser resfriado pelo dispositivo elétrico Peltier ${ }^{7}$. Resumidamente, esse dispositivo usa um

\footnotetext{
${ }^{5}$ Feedthough da MDC Vacuum Products, LLC (Type D15PIN DN50KF).

${ }^{6}$ Solid State Recirculating Chiller T255P.

${ }^{7}$ Usamos na nossa montagem um Peltier da marca Marlow Industries: Thermoeletric Cooler modelo SP2402, RoHS2002/95/EC.
} 


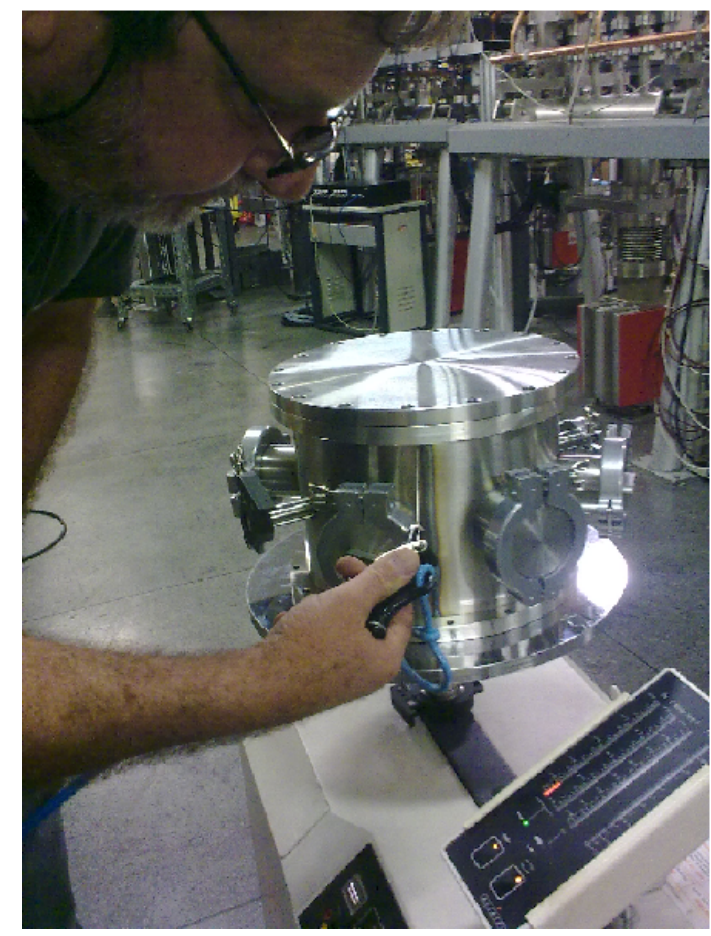

Figura 6.5: Realização do teste de vácuo. Observa-se o técnico injetando, através da ponta de prova, gás Hélio nas soldas da câmara. À direita, é visto o medidor de pressão que informa o quanto de gás vazou para dentro da câmara. O vácuo obtido com esse teste foi da ordem de $10^{-6} \mathrm{mBar}$.
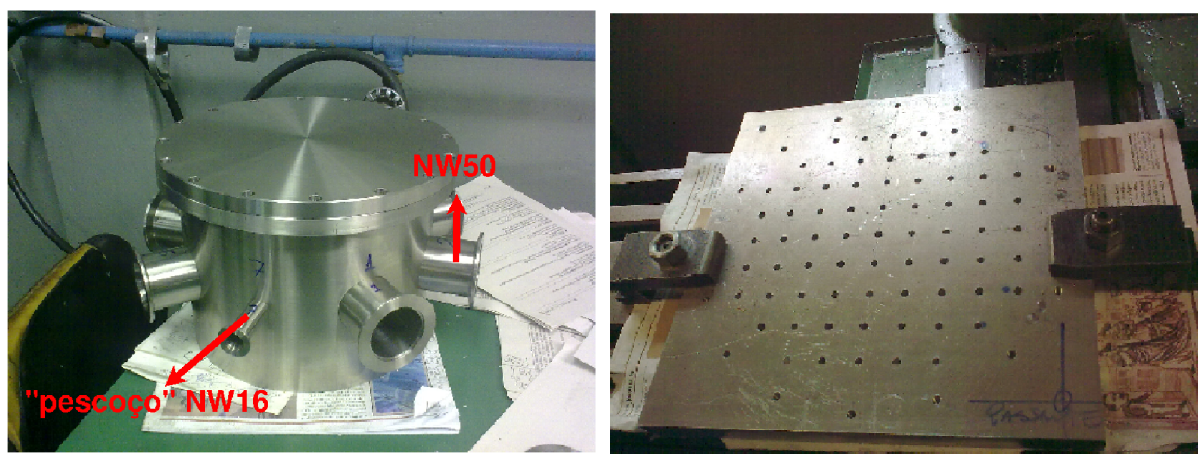

Figura 6.6: A fotografia, à esquerda, mostra o corpo e a tampa da câmara de vácuo feitas em inox. À direita, é vista a base da câmara em alumínio onde o corpo da câmara é fixado. Observa-se na base de alumínio o mesmo padrão de furação da mesa ótica. 


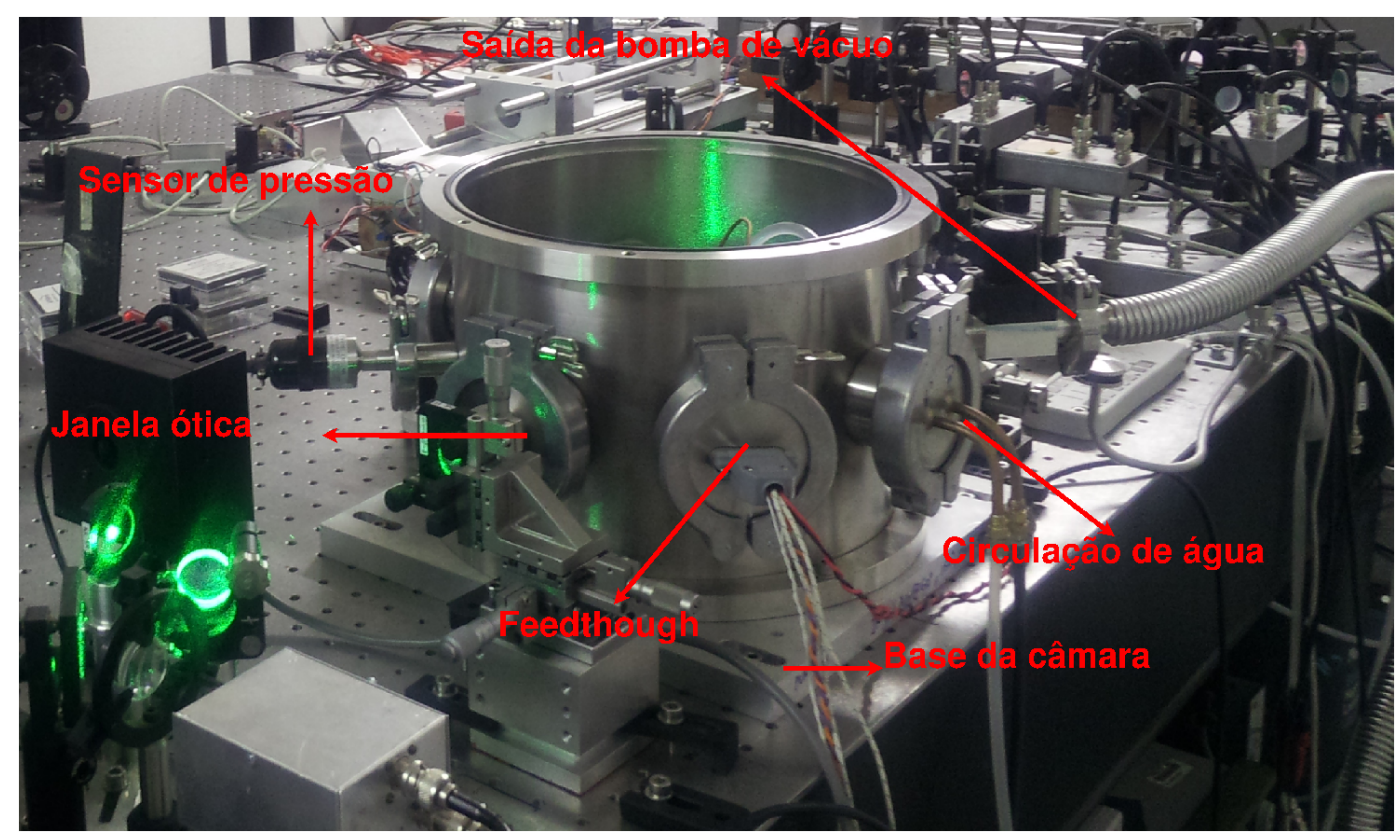

Figura 6.7: Visão geral externa da câmara de vácuo na montagem. As flanges padrão NW16 são usadas para conectar a bomba mecânica de vácuo e o sensor de pressão. E as NW50 são usadas para fixar as janelas óticas, os feedthough e a flange modificada para permitir a circulação de água dentro da câmara.
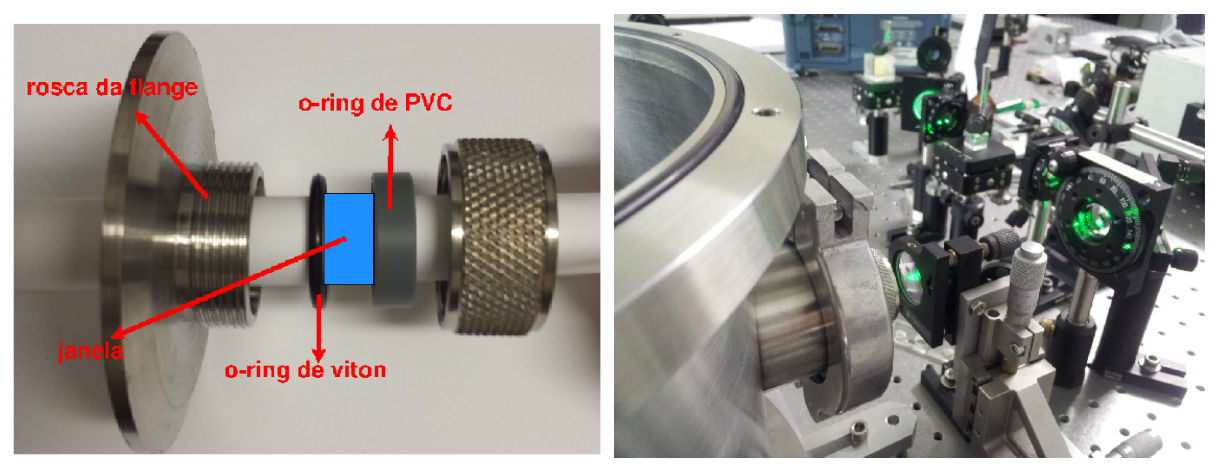

Figura 6.8: A fotografia, à esquerda, mostra o detalhe da flange de inox que abriga a janela ótica que fica localizado entre dois o-rings. À direita, é visto o perfil da flange já montada sobre um dos "pescoços" da câmara, fixada por uma abraçadeira, e sendo atravessada por um feixe. 
efeito físico de mesmo nome para produzir um gradiente de temperatura mediante a passagem de corrente elétrica através dele. Como o Peltier é uma máquina térmica, quanto menor é a temperatura da sua fonte fria, maior será a temperatura da sua fonte quente. Assim, é necessário também resfriar a fonte quente do Peltier para atingirmos temperaturas cada vez menores do cristal. Esse sistema de resfriamento é realizado através de uma peça de cobre onde, internamente, circula água bombeada pelo Chiller. A água entra e sai da câmara de vácuo através de uma flange NW50 modificada com tubos de cobre que a atravessam soldadas.

Para facilitar a troca de calor entre a fonte fria e o cristal e tornar a sua temperatura homogênea, construiu-se ainda uma peça de cobre, uma espécie de "forno", que abriga o cristal. Esse forno é ilustrado também na figura 6.9.

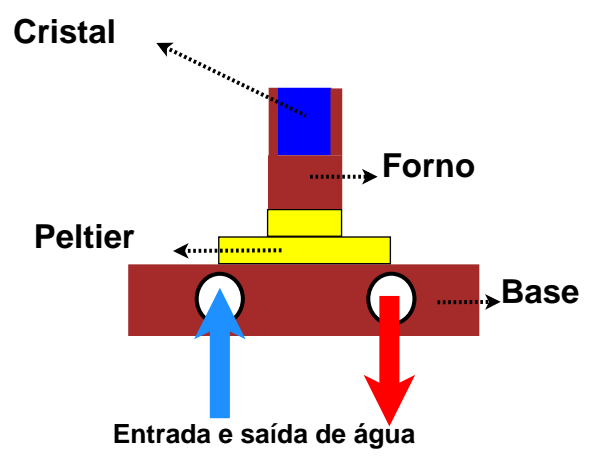

Figura 6.9: Esquema da montagem mecânica do sistema de refrigeração do cristal KTP. Há circulação de água na base de cobre(em marrom) para resfriar a fonte quente do Peltier(amarelo). A água é bombeada de fora da câmara pelo Chiller e adentra à mesma através de uma flange NW50 adaptada para passagem de água. O cristal(azul) encontra-se localizado em um "forno de cobre" (marrom) e em contato com a fonte fria do Peltier.

Além de projetar um sistema de refrigeração do cristal, precisamos também criar um mecanismo para mudar a posição do cristal que pudesse ser controlado eletricamente do lado de fora na câmara. Esse grau de liberdade a mais no alinhamento do OPO permite atingir condições de ressonância cada vez mais otimizadas. Esse mecanismo foi construído adaptando-se a montagem cristal + Peltier + peça de cobre a um suporte ${ }^{8}$ motorizado para espelho da marca Newport que tínhamos no laboratório.

Para fixar esse suporte motorizado e fornecer ainda mais graus de liber-

\footnotetext{
${ }^{8}$ Piezo motor driven optical mounts (AG-M100N) da Newport.
} 
dade na movimentação do cristal, usamos dois suportes para transladar ${ }^{9} \mathrm{e}$ rotacionar ${ }^{10}$ toda a montagem descrita. A Figura 6.10 exibe a adaptação do suporte motorizado, assim como a visão geral da montagem com o uso do transladador e o rotacionador.

Todos os suportes dos elementos óticos(espelhos e cristal) e da peça de cobre são fixados à base de alumínio da câmara de vácuo respeitando o alinhamento do feixe de bombeio. Depois de montar o esquema ilustrado na Figura 6.10, posiciona-se os suportes que sustentam os espelhos do OPO. Como será discutido mais adiante, tivemos muita dificuldade com um ruído de natureza mecânica que interferiu no travamento das cavidades óticas. Para minimizar esse efeito, a montagem mecânica do suporte dos espelhos passou por algumas modificações ao longo do tempo. Atualmente, o espelho de entrada do OPO está completamente fixado em uma peça em alumínio fixada a um poste de alumínio parafusado à base da câmara de vácuo. O espelho de saída está montado em um PZT ${ }^{11}$ fixado a um suporte da ThorLabs ${ }^{12}$. Esse suporte também está preso a um outro poste de alumínio parafusado à base da câmara. A Figura 6.11 exibe uma fotografia da montagem interna da câmara.

\subsection{O canal clássico EOM e a sua caracte- rização}

De acordo com o protocolo do teletransporte em variáveis contínuas, após a realização das medidas, Alice transmite, por meio de cabos, os seus resultados para Bob, que os utiliza para reconstruir o estado coerente com modulações produzido por Victor.

Como essas medidas resultam em flutuações nas fotocorrentes produzidas nos detectores, é necessário que Bob imprima tais informações nas quadraturas de um feixe coerente, para que, juntamente com o feixe emaranhado, ele possa refazer o estado coerente inicial. Tal feito só é possível com o uso de dispositivos, conhecidos como moduladores eletro- óticos (EOM), capazes de alterar algumas propriedades intrínsecas da luz que o atravessa.

Esses dispositivos, juntamente com os cabos, fazem parte do canal clássico do processo descrito no capítulo anterior. Conforme visto na seção 4.5.3, Bob

\footnotetext{
${ }^{9}$ Microblock 3-Axis Positioner with Fine Thread Thumbscrew(MBT602/M) da ThorLabs.

${ }^{10}$ Pitch and Yaw Platform with Thumbscrew Drives(APY001/M) da ThorLabs.

${ }^{11}$ Low voltage ring actuators without casing HPSt 150/14-10/25 da Piezomechanik GmbH.

${ }^{12}$ Polaris Kinematic Mounts K-05 da ThorLabs.
} 


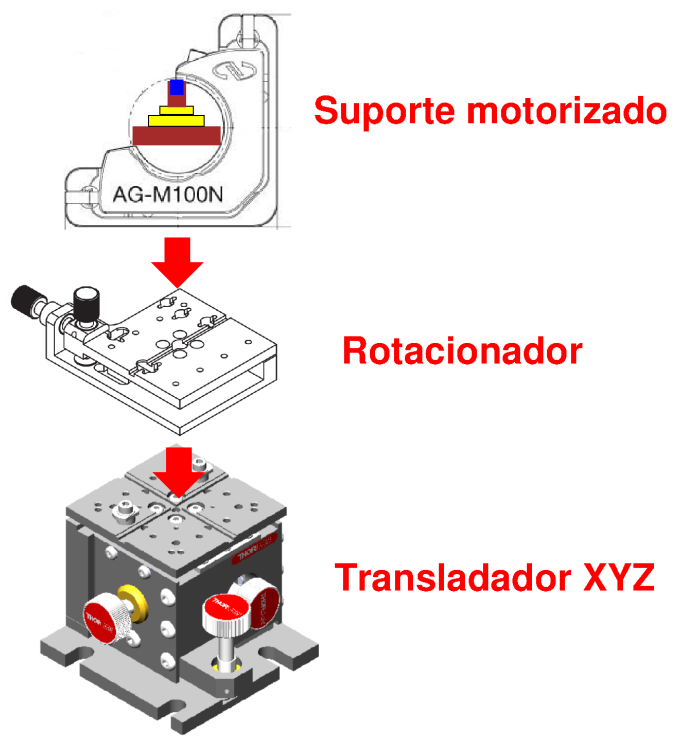

Figura 6.10: Visão da montagem do circuito de água(base de cobre), Peltier e cristal(o padrão de cores é o mesmo da figura 6.9) adaptado ao suporte motorizado fixado ao rotacionador, que está fixado ao transladador XYZ. O sistema inteiro é fixado à base da câmara de vácuo através do transladador.

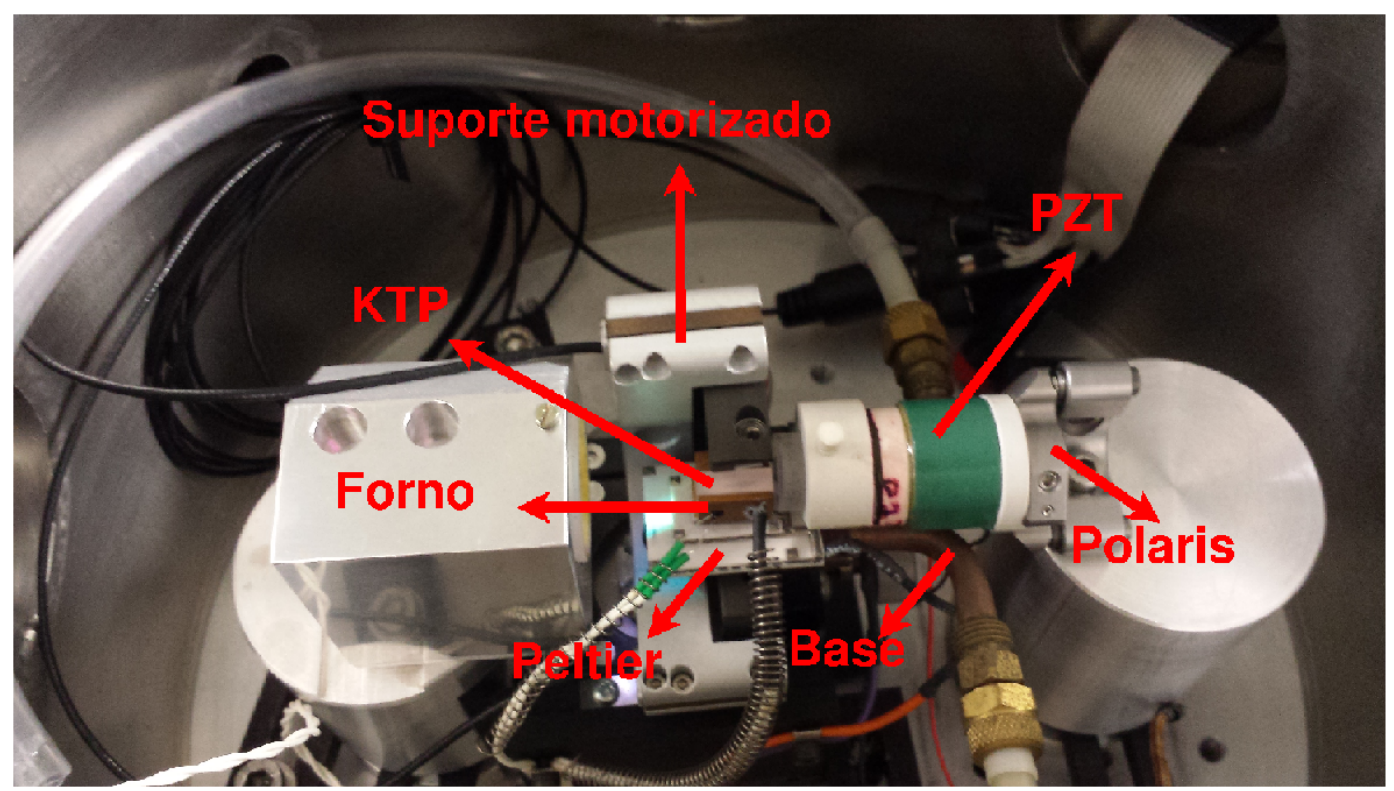

Figura 6.11: Esquema atual da montagem mecânica do OPO no interior da câmara de vácuo. 
consegue transformar as fotocorrentes recebidas de Alice em deslocamento de um estado coerente, dependendo do ganho do dispositivo usado por ele, no caso, o EOM. Assim, faz-se necessário a caracterização do ganho dos EOMs que serão utilizados na implementação do teletransporte.

As próximas seções tratam desses dispositivos e de como foi feita a caracterização dos quatro EOMs adquiridos pelo LMCAL.

\subsubsection{Moduladores eletro-óticos}

Quando uma onda eletromagnética atravessa certos tipos de cristais submetidos a um potencial elétrico, algumas de suas propriedades, como por exemplo a fase, alteram-se, devido a um efeito denominado efeito eletro-ótico. Esse efeito é utilizado na construção de dispositivos, como o EOM, que visam modular alguma das propriedades da onda eletromagnética e, dessa forma, transferir-lhe a informação contida na variação de potencial elétrico.

Ao submeter um cristal a um potencial elétrico $V$, sua estrutura cristalina é levemente alterada, causando uma mudança no seu índice de refração [64]. Em certos tipos de cristais que não possuem um centro de simetria, como o $K D P$, o $\mathrm{LiNbO}_{3}$ e $\mathrm{BaTiO}_{3}$, a dependência do índice de refração com o campo elétrico aplicado $E^{\prime}$ é linear. Essa relação é conhecida como efeito eletro-ótico linear ou efeito Pockels, em homenagem ao pesquisador que o estudou em 1893 [64].

Um modulador eletro-ótico é um equipamento composto basicamente de um cristal sem centro de simetria, situado entre duas placas de eletrodos, formando um capacitor. O campo elétrico aplicado é uniforme ao longo de todo o comprimento do cristal, o que resulta em uma mudança no índice de refração também uniforme ao longo do cristal. Se o campo $E^{\prime}$ é aplicado ao cristal na mesma direção de propagação da onda, o EOM é do tipo longitudinal, porém se $E^{\prime}$ for aplicado na direção perpendicular à propagação da onda, este é do tipo transverso.

O EOM pode modular de forma controlada a fase, a polarização, a amplitude, frequência e direção de propagação de uma onda eletromagnática a depender da configuração em que se encontre junto a outros elementos óticos, tais como polarizadores, espelhos e BS. A Figura 6.12 ilustra um EOM longitudinal, cujo cristal, de dimensões $l$, $d$, é submetido a uma diferença de potencial $V$ e modula a fase de uma onda eletromagnática.

Geralmente, a tensão aplicada ao cristal gira o seu eixo principal [64]. Para modular somente a fase da onda, é preciso incindir o campo com o vetor de polarização orientado na mesma direção do eixo principal. A Figura 6.12 mostra uma configuração para modular a fase da onda em que se usa um polarizador antes do EOM para orientar adequadamente o vetor de 


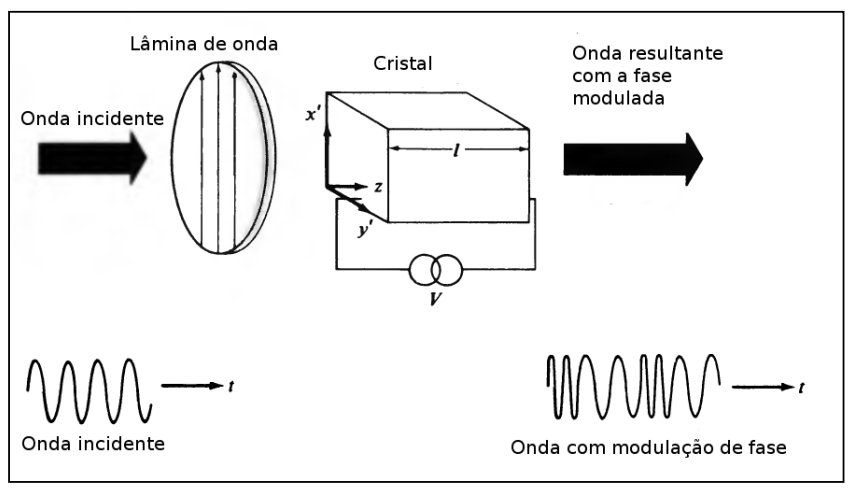

Figura 6.12: Figura adaptada de [64] que ilustra esquematicamente a atuação de um EOM longitudinal em uma configuração para modular a fase do campo. A onda incide em um polarizador, ou lâmina de onda, para que o seu vetor de polarização se alinhe na direção do eixo principal do cristal, no caso eixo $x^{\prime}$. O cristal do EOM está submetido a um potencial elétrico $V$. Após a passagem do campo através do cristal, há uma mudança na sua fase que depende da função de $V$ no tempo.

polarização.

O campo elétrico incidente ${ }^{13}$ é descrito matematicamente de acordo com a equação (2.1), considerando que somente há propagação no sentido $+z$ :

$$
\mathbf{E}(\mathbf{r}, t)=E(t) e^{i(k r-\omega t)} \mathbf{z}
$$

em que o vetor de onda $\mathbf{k}$ é paralelo à direção de propagação da onda e igual a:

$$
k=\frac{n \nu}{c}=\frac{2 \pi n}{\lambda}
$$

A diferença de fase que aparece em (6.9), denotada por $\Delta \phi$, origina-se no termo $k r$ da equação (6.10). Como o cristal eletro-ótico está submetido a um campo elétrico $E^{\prime}$, há uma mudança no seu índice de refração $n^{\prime}$, que pode ser escrita como:

$$
n^{\prime}=n+\Delta n
$$

Assim, $\Delta \phi$ é calculado substituindo-se (6.11) em (6.10) e multiplicando o $k$ obtido por $l$ :

$$
\Delta \phi=k r=\frac{2 \pi}{\lambda} l n^{\prime}=\frac{2 \pi}{\lambda} l(n+\Delta n)=\phi+\Delta \phi
$$

O primeiro termo de (6.10), $\phi$, refere-se ao termo que independe da aplicação do campo elétrico $E^{\prime}$, porém o segundo termo, $\Delta \phi$, surge exatamente com a

\footnotetext{
${ }^{13}$ Considera-se a fase da onda inicialmente igual a zero.
} 
aplicação da tensão. A relação entre a mudança de índice de refração $\Delta n$ e campo elétrico no efeito eletro-ótico é linear. Dessa forma, a expressão para $\Delta n$ é escrita como:

$$
\Delta n=k_{\text {cristal }} E^{\prime},
$$

$k_{\text {cristal }}$ corresponde ao coeficiente eletro-ótico do cristal.

Sabendo-se que, no caso do EOM longitudinal, $E^{\prime}=\frac{V}{l}$ e substituindo a equação (6.13) na (6.11), obtém-se $\Delta \phi$ :

$$
\Delta \phi_{\text {longitudinal }}=\frac{2 \pi k_{\text {cristal }} V}{\lambda}
$$

No caso do EOM transverso, $E^{\prime}=\frac{V}{d}$ e $\Delta \phi$ é dado por:

$$
\Delta \phi_{\text {transverso }}=\frac{2 \pi k_{\text {cristal }} l V}{\lambda d}
$$

A voltagem capaz de produzir uma mudança de fase em (6.9) igual a $\pi$ é chamada de voltagem de meia onda, $V_{\pi}$, e depende essencialmente do cristal que compõe o EOM. Para o EOM longitudinal, $V_{\pi}$ é igual a:

$$
V_{\pi(\text { longitudinal })}=\frac{\lambda}{2 k_{\text {cristal }}}
$$

e para o transverso:

$$
V_{\pi(\text { transverso })}=\frac{\lambda d}{2 k_{\text {cristal }} l} .
$$

O LMCAL possui quatro unidades de EOMs do tipo transverso da marca comercial Linus. Duas dessas unidades são da série LM0202 e as outras duas da série PM25. Na próxima seção, descreve-se como foi feita a caracterização desses dois tipos de moduladores usando um interferômetro de Mach-Zehnder.

\subsection{Caracterização dos EOMs}

Para caracterizar os EOMs do LMCAL, inicialmente, montou-se um interferômetro de Mach-Zehnder(MZ) e, em seguida, em um dos seus braços, fixou-se os EOMs, primeiro o da série LM0202 e depois o PM25. O objetivo foi medir a modulação de fase adquirida por um campo ao atravessar o EOM como função da tensão que é submetido o modulador.

Visando um completo entendimento dos resultados da medidas, é necessário, antes, analisar teoricamente o MZ nas duas situações: sem um EOM em um dos seus braços, e com um EOM em um de seus braços, submetido 
a uma tensão $V$ variável no tempo. Na próxima seção será descrito teoricamente o interferômetro de $\mathrm{MZ}$ e a influência do EOM sobre a modulação de fase do campo que o atravessa. Na seção seguinte, será descrito todo o procedimento experimental adotado na montagem do MZ, assim como na caracterização dos EOMs.

\subsubsection{Interferômetro de Mach-Zehnder (MZ)}

Um interferômetro de Mach-Zehnder consiste de um arranjo de dois BS e dois espelhos posicionados de forma simétrica, como visto na Figura 6.13. Os coeficientes de reflexão e de transmissão de amplitude dos dois BS são iguais, por hipótese, e denotados por $(r, t)$. Os coeficiente de reflexão de amplitude de ambos os espelhos da montagem são iguais a um. O campo

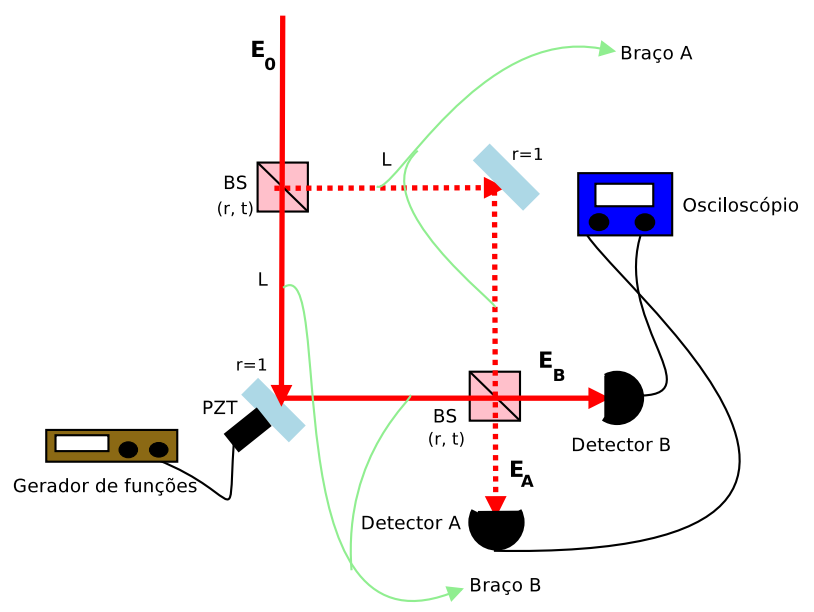

Figura 6.13: Figura que ilustra um interferômetro de Mach-Zehnder. O campo incidente $\mathbf{E}_{0}$, ao atravessar o primeiro BS, divide-se em dois feixes e origina os "braços" A e B do interferômetro. Os detectores A e B detectam os campos $\mathbf{E}_{A}$ e $\mathbf{E}_{B}$ respectivamente.

incidente no $\mathrm{MZ}$, denotado por $\mathbf{E}_{0}$, após atravessar o primeiro BS divide-se em dois outros feixes que dão origem a dois "braços" no interferômetro vistos na Figura 6.13: "braço" A e "braço" B.

Se os "braços" têm exatamente o mesmo comprimento, não há interferência entre os campos vindos dos "braços" A e B, entretanto, se existe uma diferença entre os caminhos da ordem do comprimento de onda da luz, isso provoca uma diferença de fase entre os campos e eles se interferem em ambos os detectores. Para gerar, experimentalmente, tal diferença de caminho, é preciso acoplar um dos espelhos a um PZT. 
Outra forma de variar a fase entre os campos, além da diferença de caminho entre os "braços", consiste em inserir algum dispositivo com um índice de refração diferente em um dos "braços" do MZ.

Na Figura 6.13, o campo resultante da interferência entre os campos provenientes de ambos os "braços" e detectado por A, é chamado de $\mathbf{E}_{A}$, e o detectado por B, $\mathbf{E}_{B}$.

A fase adquirida pelo campo ao percorrer a distância $L$, segundo a equação (6.9), é dada por:

$$
\delta=k r=k L=\frac{2 \pi L}{\lambda}
$$

Assumindo que o campo $\mathbf{E}_{0}$, incidente no $\mathrm{MZ}$, é do tipo monocromático e polarizado linearmente, pode-se escreve o campo $\mathbf{E}_{A}$, de posse de (6.18), como:

$$
\mathbf{E}_{A}=2 \operatorname{tr} \mathbf{E}_{0} e^{i(\omega t+2 \pi+2 \delta)},
$$

lembrando que, a cada reflexão, o campo adquire uma fase $\pi$.

De forma similar, o campo $\mathbf{E}_{B}$ pode ser escrito matematicamente por:

$$
\mathbf{E}_{B}=t^{2} \mathbf{E}_{0} e^{i(\omega t+\pi+2 \delta)}+r^{2} \mathbf{E}_{0} e^{i(\omega t+\pi+2 \delta)}
$$

Os detectores são instrumentos de medida sensíveis à média do fluxo do vetor de Poynting, dada pela intensidade $I$, que é proporcional ao módulo ao quadrado do campo em questão, a menos de um fator de ganho ${ }^{14}$. Dessa forma, calcula-se a intensidade $I_{A}$ do campo $\mathbf{E}_{A}$, dado por (6.19):

$$
I_{A}=\mathbf{E}_{A}^{*} \cdot \mathbf{E}_{A}
$$

Sabendo-se que $r^{2}=R$ e $t^{2}=T, R$ e $T$ coeficientes de reflexão e transmissão respectivamente, a equação (6.21) resulta em:

$$
I_{A}=4 I_{0} t^{2} r^{2}=4 I_{0} T R
$$

em que $I_{0}$ é a intensidade do campo incidente $\mathbf{E}_{0}$. Considerando um BS do tipo $50: 50, R=T=0,5, \operatorname{logo} I_{A}=1$. Isso significa que toda a intensidade inicial $I_{0}$ é detectada em A e nenhuma no detector $\mathrm{B}$, no caso específico onde os dois "braços" do MZ tem o mesmo comprimento $L$.

Entretanto, se há alguma diferença de fase $\phi$ entre os campos, provocada ou pela diferença de comprimento entre os "braços" ou pela presença de algum dispositivo que altera o índice de refração do meio, o campo $\mathbf{E}_{A}$ passa a ser escrito como:

$$
\mathbf{E}_{A}=\mathbf{E}_{0} \operatorname{tr}\left[e^{i(\omega t+2 \pi+2 \delta)}+e^{i(\omega t+2 \pi+2 \delta+\phi)}\right],
$$

\footnotetext{
${ }^{14}$ Para simplificar, o ganho é considerado igual a um.
} 
cuja intensidade é:

$$
I_{A}=I_{0}\left[2 t^{2} r^{2}+t^{2} r^{2} e^{i \phi}+t^{2} r^{2} e^{-i \phi}\right]
$$

Como $e^{i \phi}=\cos \phi+i \sin \phi$, a equação (6.24) é simplificada:

$$
I_{A}=2 I_{0} t^{2} r^{2}(1+\cos \phi)=2 T R(1+\cos \phi)
$$

Para $R=T=0,5,(6.25)$ torna-se igual a:

$$
I_{A}=0,5 I_{0}(1+\cos \phi)
$$

Da equação (6.29), é possível observar que a intensidade do campo $\mathbf{E}_{A}$, detectada em A, depende da fase relativa entre os campos $\mathbf{E}_{A}$ e $\mathbf{E}_{B}$. Resumidamente, para valores de $\phi$ iguais a $0, \frac{\pi}{2}, \pi$, a intensidade detectada em A:

$$
I_{A}=\left\{\begin{array}{l}
I_{0}, \text { se } \phi=0 \\
\frac{I_{0}}{2}, \text { se } \phi=\frac{\pi}{2} \\
0, \text { se } \phi=\pi
\end{array}\right.
$$

Por outro lado, a intensidade detectada em B está em quadratura com $I_{A}$ :

$$
I_{B}=I_{0}-I_{A}=0,5 I_{0}(1-\cos \phi)
$$

Como a intensidade do campo recebida por um detector resulta em uma diferença de potencial, expressar-se-á ao longo desse texto sempre a tensão resultante no detector:

$$
V=k_{\text {detector }} I
$$

em que $k_{\text {detector }}$ é o ganho do detector que, para simplificar, será sempre considerado igual a 1. Assim, as equações das tensões nos detectores A e B, resultado da incidência dos campos $\mathbf{E}_{A}$ e $\mathbf{E}_{B}$, são, respectivamente, iguais a:

$$
\begin{aligned}
& V_{A}=0,5 V_{0}(1+\cos \phi), \\
& V_{B}=0,5 V_{0}(1-\cos \phi) .
\end{aligned}
$$

É observado na figura (6.14) um gráfico das tensões medidas pelos detectores A e B, vistos na figura (6.13), em função da fase $\phi$. 


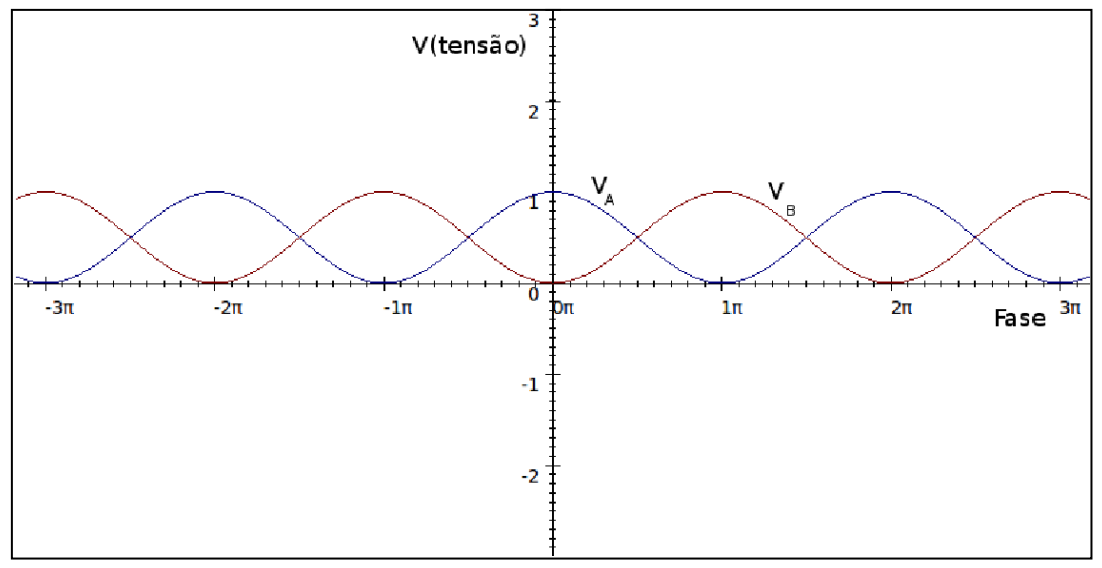

Figura 6.14: Gráfico das funções (6.29) e (6.30) da tensão nos detectores A e B respectivamente como função da fase $\phi$. Considerou-se $V_{0}=1$.

\subsubsection{Interferômetro de MZ com um EOM}

A Figura 6.15 ilustra o esquema da nova configuração obtida para o interferômetro de Mach-Zehnder ao se inserir um modulador no esquema da Figura 6.13. Com a presença do EOM, a fase $\phi$, na equação (6.29), entre os campos $\mathbf{E}_{A}$ e $\mathbf{E}_{B}$ é igual a fase devido a diferença de comprimentos entre os "braços" A e B, $\phi_{\text {diff }}$, mais a diferença de fase induzida pelo EOM, $\phi_{E O M}$, com a variação do seu índice de refração:

$$
\phi=\phi_{d i f}+\phi_{E O M}
$$

Supondo que a tensão aplicada sobre o EOM, $V$, seja uma função senoidal:

$$
V=A \sin (2 \pi \nu t)
$$

em que $A$ é amplitude e $\nu$ a frequência do sinal, a diferença de fase, ou modulação de fase, $\phi_{E O M}$, é dada pela equação (6.15):

$$
\phi_{E O M}=k_{E O M} A \sin (2 \pi \nu t),
$$

em que a constante $k_{E O M}$ depende das características eletro-óticas do modulador em questão. Segundo as equações (6.14) e (6.15), (6.16) e (6.17), os $k_{E O M}$ dos EOM dos tipos longitudinal e transverso são, respectivamente, iguais a:

$$
k_{E O M(\text { longitudinal })}=\frac{2 \pi k_{\text {cristal }}}{\lambda}=\frac{\pi}{V_{\pi(\text { longitudinal })}}
$$




$$
k_{E O M(\text { transverso })}=\frac{2 \pi k_{\text {cristal }} l}{\lambda d}=\frac{\pi}{V_{\pi(\text { transverso })}}
$$

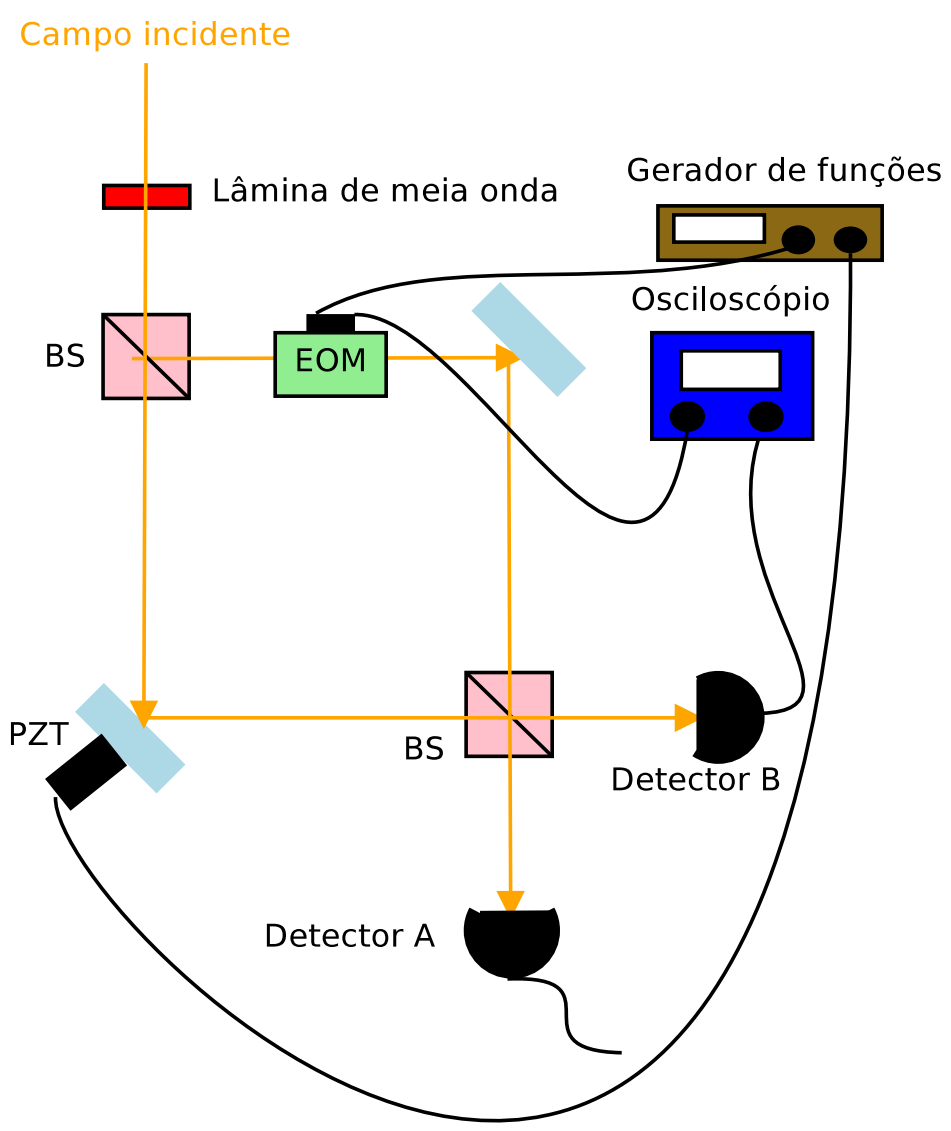

Figura 6.15: Figura que ilustra o interferômetro de Mach-Zehnder usado para caracterizar os EOMs. O feixe incide no primeiro BS e, em seguida, divide-se em dois "braços". Em um deles, encontra-se o EOM ligado a um gerador de funções.

Em uma situação em que $\phi_{\text {dif }}=0$ no interferômetro de MZ, a fase $\phi$ da equação (6.29) é provocada unicamente pela tensão (6.32) aplicada ao EOM e, a tensão no detector A torna-se:

$$
V_{A}=0,5 V_{0}\left[1+\cos \left(k_{\text {eom }} A \sin (2 \pi \nu t)\right]\right.
$$

Como $k_{E O M}=\frac{\pi}{V_{\pi}}$, é conveniente analisar o comportamento da função (6.36) para certos valores $\left(V_{\pi}, \frac{V_{\pi}}{2}\right.$ e $\left.\frac{V_{\pi}}{4}\right)$ da amplitude $A$. Os gráficos da Figura 6.16 ilustram a função (6.36) para diferentes valores de $A$. 
No caso geral em que o PZT está ligado e EOM submetido a tensão (6.32), a expressão para a tensão $V_{A}$ é calculada substituindo-se a equação (6.31) na equação (6.29):

$$
V_{A}=0,5 V_{0}\left[1+\cos \left(\phi_{d i f}+\phi_{E O M}\right)\right]
$$

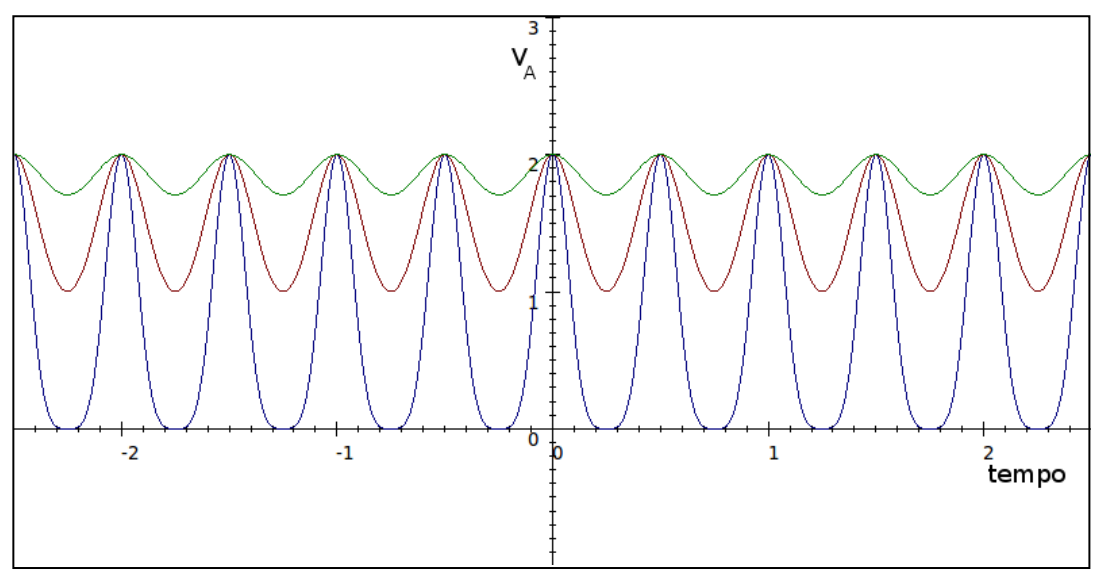

Figura 6.16: Gráfico da função 6.36 para três valores de amplitude $A$ do sinal enviado ao EOM. Na curva verde, $A=\frac{V_{\pi}}{4}$, na vermelha, $A=\frac{V_{\pi}}{2} \mathrm{e}$ na azul $A=V_{\pi}$. À medida que a amplitude do sinal aumenta, as curvas se aproximam do eixo do tempo. O valor da amplitude no qual a curva toca esse eixo é exatamente $V_{\pi}$ do modulador em questão. Nas três curvas, $0,5 V_{0}=1$.

Para facilitar a notação, considera-se $0,5 V_{0}=\overline{V_{A}}$ e a equação (6.37) torna-se:

$$
V_{A}=\overline{V_{A}}\left[1+\cos \left(\phi_{\text {dif }}+\phi_{E O M}\right)\right]
$$

A equação (6.38) pode ser reescrita como:

$$
V_{A}=\overline{V_{A}}\left[1+\cos \phi_{d i f} \cos \phi_{E O M}-\sin \phi_{\text {dif }} \sin \phi_{E O M}\right]
$$

Para pequenas modulações de fase provocadas pelo EOM, valem as seguintes aproximações:

$$
\begin{gathered}
\sin \phi_{E O M}=\phi_{E O M}-\frac{\phi_{E O M}^{3}}{3 !}+\ldots \approx \phi_{E O M} \\
\cos \phi_{E O M}=1-\frac{\phi_{E O M}^{2}}{2 !}+\ldots \approx 1
\end{gathered}
$$

Usando-se as aproximações (6.40) e (6.41) na equação (6.39): 


$$
V_{A}=\overline{V_{A}}\left[1+\cos \phi_{d i f}-\phi_{E O M} \sin \phi_{d i f}\right]
$$

Quando não há modulação $\phi_{E O M}$, a diferença de fase entre os campos é resultado somente da diferença de caminho entre os "braços" A e B do MZ e, de acordo com 6.42 , é igual a:

$$
\phi_{d i f}=\operatorname{arcos}\left(\frac{V-\overline{V_{A}}}{\overline{V_{A}}}\right),
$$

em que $V$ é a tensão de leitura no detector que corresponde uma diferença de fase de $\phi_{\text {dif }}$, no caso em que $\phi_{E O M}=0$. Segundo a equação $(6.43), \phi_{\text {dif }}$ será igual $\mathrm{a}^{15} \pm \frac{n \pi}{2}$ sempre que for atingida a condição $V=\overline{V_{A}}$. Na montagem experimental, descrita a seguir, travou-se o MZ montado exatamente nessa condição.

Assim, garantida a condição $\phi_{\text {dif }}= \pm \frac{n \pi}{2}$, atingida quando $V=\bar{V}_{A}$, a modulação presente no MZ, se existir, será causada apenas pelo EOM e, a equação (6.42), resulta em:

$$
V_{A}=\overline{V_{A}}\left[1 \pm \phi_{E O M}\right]
$$

A modulação de fase em um campo provocada pelo EOM, submetido à uma tensão variável no tempo de amplitude muito menor que sua voltagem de meia onda, é:

$$
\phi_{E O M}= \pm \frac{\left(V_{A}-\overline{V_{A}}\right)}{\overline{V_{A}}}
$$

Na próxima seção, será descrito o procedimento experimental adotado para montar o MZ, esquematizado na Figura 6.15, travá-lo em $\phi_{\text {dif }}= \pm \frac{\pi}{2} \mathrm{e}$ para a realização das medidas $\overline{V_{A}}$ e $V_{A}-\overline{V_{A}}$.

\subsubsection{Montagem experimental e resultados}

Os EOMS da Linus das séries LM0202 e PM25 são feitos ${ }^{16}$ para operar na faixa de $1064 \mathrm{~nm}$. Para caracterizá-los experimentalmente, foi montado um interferômetro de $\mathrm{MZ}$, devidamente alinhado $^{17}$, operando nessa faixa. $\mathrm{Na}$ Figura 6.17, é vista uma foto da montagem experimental, no caso particular, em que o EOM presente era o PM25.

\footnotetext{
${ }^{15} n$ é um número inteiro.

${ }^{16}$ A transmissão do LM0202 @1064nm é 0,968 e a transmissão do PM25 é 0,966.

${ }^{17} \mathrm{O}$ critério usado para o alinhamento consistiu em medir o contraste do MZ [64]. Quanto mais próximo de 1 é o contraste mais alinhado o interferômetro está. Todas as medidas foram realizadas com o contraste do MZ maior ou igual a 0,9.
} 
Antes do interferômetro foi colocada uma roda de filtro para não saturar os detectores e uma lâmina de meia onda para controlar a polarização do campo de entrada, de forma que esta fosse paralela a algum dos eixos principais do cristal do EOM. Na saída, foram usados dois tipos de detectores: um DET36A/M(Thor Labs) e um detector, descrito anteriormente, com duas saídas: DC e HF usado para as medidas de $V_{A}$ da equação (6.45).

Um dos espelhos do MZ foi montado sobre um PZT da marca Piezomechanik da série HPSt 150/14-10/12. O PZT era o dispositivo responsável pelo deslocamento de um dos espelhos do MZ de forma a alterar o comprimento de um dos seus "braços", dando origem a fase $\phi_{\text {dif }}$.

O sinal de tensão enviado ao PZT era do tipo rampa com amplitude $3 V_{p p}$, frequência $10 \mathrm{~Hz}$, e era fornecido por um gerador de funções da marca Hewlett Packard da série 33120A. Como o PZT é um dispositivo que opera somente quando alimentado com tensões em torno de $70 \mathrm{~V}$, foi usado um amplificador da marca Thorlabs do tipo 3-Axis Piezo Controler Model MDT6960.

O travamento do MZ na condição em que a fase $\phi_{d i f}= \pm \frac{\pi}{2}$ era feito com o auxílio de um circuito eletrônico que recebia os sinais DC dos detectores A e B. A saída desse circuito era ligada ao PZT antes da realização da medida de $\phi_{E O M}$.

Antigida a condição de travamento do MZ, ligava-se o EOM a um amplificador da marca Mini-Circuits da série ZHL 1-2W e este a um gerador de funções da marca Agilent Technologies da série 81150A, que fornecia sinais periódicos em alta tensão (em torno de $100 \mathrm{MHz}$ ) com grande precisão.

Para monitorar os sinais DC e HF do detector usado e medir $\overline{V_{A}}$ e $V_{A}-\overline{V_{A}}$ foi usado um osciloscópio de 4 canais da marca Agilent Technologies da série DSO1004A. Duas pontas de prova foram usadas nas medidas: uma ligada ao detector A para medir $V_{A}$ e outra ao EOM.

Realizava-se a medida com o auxílio da função "stop" do osciloscópio. O estudo foi feito para frequências no intervalo de $10 \mathrm{MHz}-20 \mathrm{MHz}$. Para cada valor de frequência, variava-se a amplitude $(\mathrm{em} \mathrm{dBm})$ do sinal, do tipo (6.32), do gerador de sinais da Agilent e media-se com as pontas de prova: a amplitude do sinal sobre o EOM e os valores máximo e mínimo do sinal HF do detector. A amplitude, $\left(V_{A}-\overline{V_{A}}\right.$ considerada é a média desses valores máximo e mínimo. Como as modulações produzida pelos EOMs eram pequenas ${ }^{18}$, usou-se a equação (6.45) para determinar $\phi_{E O M}$.

Os gráficos das Figuras 6.18 e 6.19 ilustram as curvas de ganho dos EOMs LM0202 e PM25, obtidas experimentalmente para $15 \mathrm{MHz}$, que será a frequência de operação dos EOMs no esquema proposto para o teletransporte.

\footnotetext{
${ }^{18}$ As tensões de meia onda do LM0202 e do PM25 são em torno de 310V.
} 


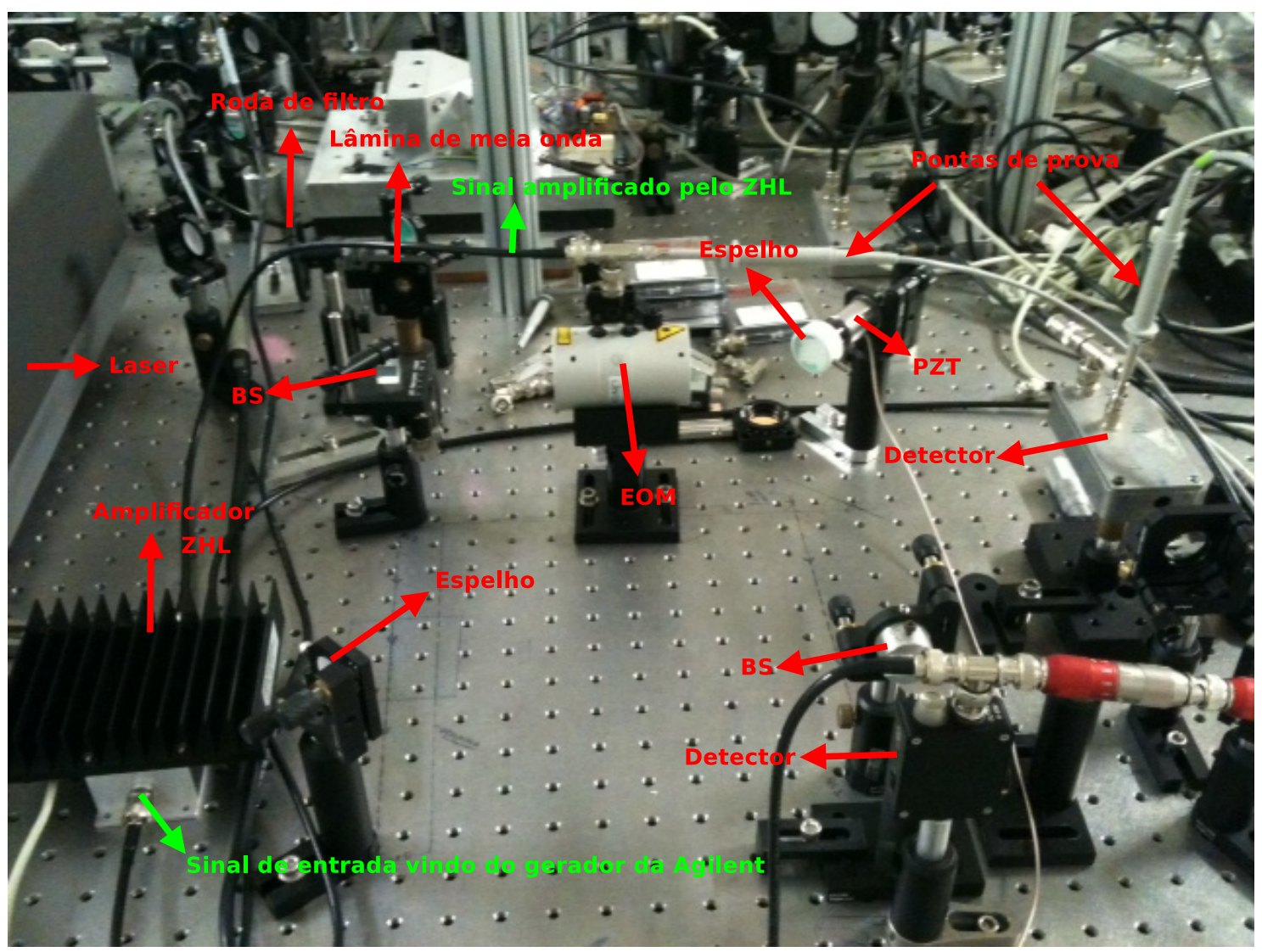

Figura 6.17: Figura ilustrativa da montagem experimental do esquema da figura 6.15 realizada no LMCAL para a caracterização do EOM da série PM25. Distância entre os elementos no MZ de aproximadamente $30 \mathrm{~cm}$. 


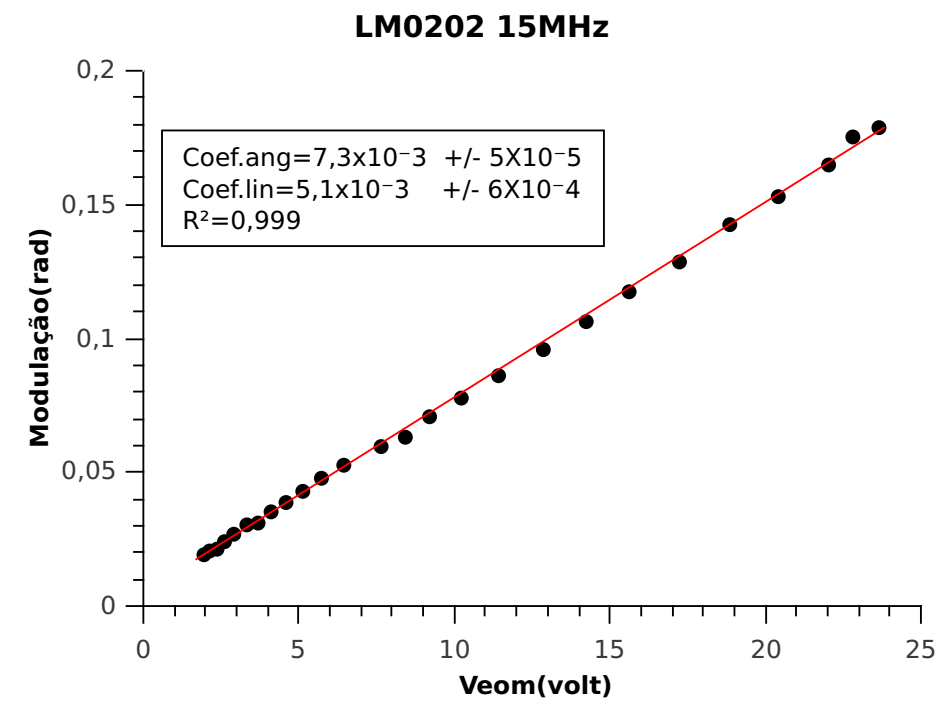

Figura 6.18: Curva de ajuste do ganho do EOM da série LM0202, operando em $15 \mathrm{MHz}$. O coeficiente angular da reta ajustada é a fase induzida pelo EOM. Para o LM0202, $\phi_{E O M}=7,3 X 10^{-3} \frac{\mathrm{rad}}{\text { volt }}$.

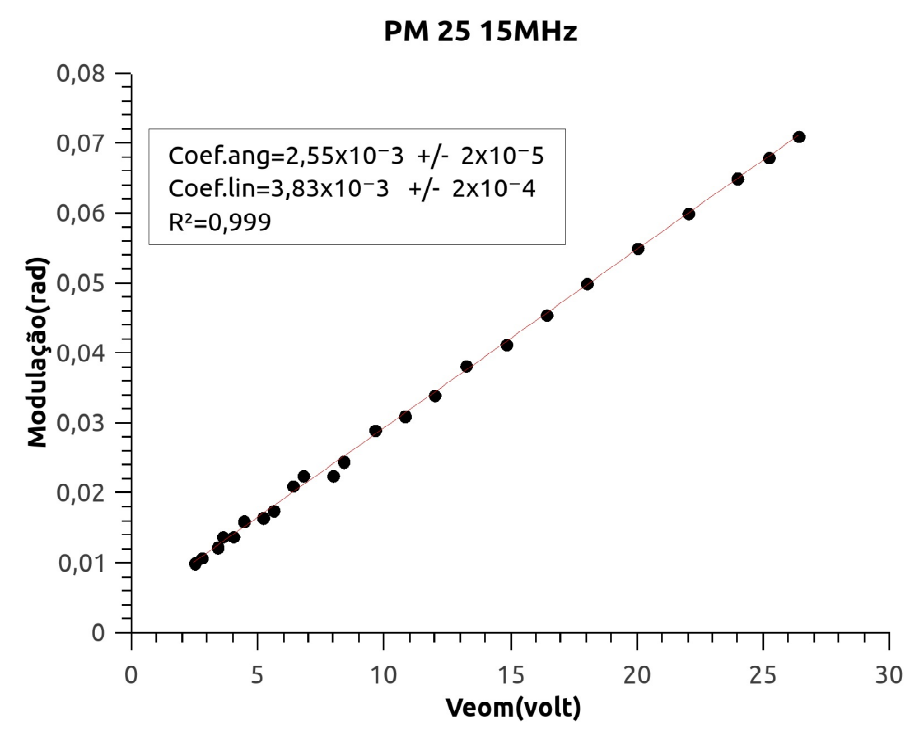

Figura 6.19: Curva de ajuste do ganho do EOM da série PM25, operando em $15 M H z$. O coeficiente angular da reta ajustada é a fase induzida pelo EOM. Para o PM25, $\phi_{E O M}=2,6 X 10^{-3} \frac{\mathrm{rad}}{\mathrm{volt}}$. 


\subsection{Construção do canal quântico: tentativas}

Conforme descrito anteriormente, o cerne da implementação do protocolo do teletrasnporte bicolor consiste em montar um OPO que gere feixes nãodegenerados com o máximo de emaranhamento possível de se alcançar experimentalmente para garantir que a fidelidade do teletransporte exceda o limite clássico de $\frac{1}{2}$.

O primeiro candidato considerado para servir de fonte do nosso protocolo foi um DROPO por produzir um estado quântico puro. Foram montados dois DROPOs, denotados nesse texto por DROPO1 e DROPO2, com diferentes configurações e cristais no interior da câmara de vácuo descrita na seção 6.2.

O DROPO1 foi completamente descartado por conter mais perdas espúrias que o desejado. Já o DROPO2 apresentou, nas primeiras medidas realizadas, o maior nível de compressão de ruído de intensidade já medidos no LMCAL, entretanto foi desconsiderado como fonte por apresentar excesso de ruído de fase que destruiu o emaranhamento entre os gêmeos.

Inviabilizada a proposta do teletransporte com um DROPO, partimos para a nossa segunda proposta com um TROPO. Nessa proposta atual, tivemos que inserir uma cavidade de filtro para eliminar o excesso de ruído de fase do laser. Foram montados dois TROPOs, TROPO1 e TROPO2, dos quais o TROPO1 foi descartado por problemas no travamento da sua cavidade. Atualmente, estamos trabalhando com o TROPO2 cujas as medidas preliminares de compressão de ruído quântico foram realizadas.

Essa seção aborda sobre todas essas tentativas de construção de fontes de emaranhamento para o teletransporte bicolor. Inicialmente, abordaremos sobre o dispositivo OPO sem adentrar nos detalhes de sua teoria, que pode ser vista em diversas referências do grupo [5, 14, 65, 66]. Em seguida, falaremos sobre os resultados experimentais obtidos com os OPOs montados que, infelizmente, mostraram-se insuficiente para a implementação do protocolo.

\subsubsection{Considerações gerais sobre um OPO}

Essa sub-seção trata, de forma resumida, sobre o gerador de emaranhamento empregado no protocolo de teletransporte, o OPO. Tanto a teoria clássica quanto a sua descrição quântica encontram-se descritas em vários artigos e teses do grupo $[5,14,65]$.

O OPO é o dispositivo mais usado em Ótica Quântica para a geração de estados comprimidos da luz. Ele é composto por um cristal de suscetibilidade não-linear inserido no interior de uma cavidade ótica, bombeado por um laser externo (denominado bombeio) de alta potência e, esquematicamente, exibido na Figura 6.5. 
Exatamente como acontece em um laser, o cristal atua como um meio de ganho, levando o sistema à oscilação e convertendo o bombeio em dois feixes, denominados gêmeos (sinal e complementar), por ser produzidos simultaneamente.

Sabemos do Eletromagnetismo clássico, que a aplicação de um campo eletromagnético a um dado meio material produz um campo de polarização induzida no mesmo. Se o meio não absorver o campo e responder linearmente ao campo aplicado, caso mais estudado nos cursos de Física básica, a polarização $\left(P_{i}\right)$ é descrita pela relação:

$$
P_{i}=\chi_{i j} A_{j}
$$

onde $\chi_{i j}$ é a suscetibilidade elétrica linear e $A_{j}$ é uma componente do campo elétrico aplicado.

Isso quer dizer que, em um meio linear, dois campos se propagam livremente sem ocorrer a interação entre eles, ou seja, os campos não trocam energia entre si. Se pensarmos em termos quânticos, esse caso resultaria somente em uma hamiltoniana sem termos de interação entre os operadores aniquilação de cada modo.

Entretanto, para campo eletromagnéticos intensos e cristais com simetria apropriada $^{19}$, a resposta não-linear do meio poder ocorrer. Assim, a suscetibilidade elétrica é expandida de forma a incluir termos de ordens mais altas e a polarização é escrita como $[5,67]$ :

$$
P_{i}=\chi_{i j}^{(1)} A_{j}+\chi_{i j k}^{(2)} A_{j} A_{k}+\ldots
$$

em que $\chi^{(n)}$ são os tensores de suscetibilidade elétrica de ordem $n$.

Com a dependência quadrática, há interferência ou acoplamento entre os campos no interior da cavidade. Se considerarmos a quantização dos campos interagindo, veremos que esse processo implica na aniquilação de um fóton de bombeio, de frequência $\omega_{0}$, e na produção de dois outros fótons com frequências $\omega_{1}$ e $\omega_{2}$, do sinal e do complementar, correlacionados quanticamente.

Nesse fenômeno de conversão de frequência, há a conservação de energia, o que implica em afirmar que a soma das frequências do sinal e do complementar resulta em:

$$
\hbar \omega_{0}=\hbar \omega_{1}+\hbar \omega_{2}
$$

Além da energia, há também a conservação de momento dos fótons dentro do cristal, denominado acordo de fase, que é igual a, no caso de um

\footnotetext{
${ }^{19} \mathrm{~A}$ resposta de segunda ordem ocorrem em materiais ditos "noncentrosymmetric".
} 
acoplamento perfeito entre os campos:

$$
\mathbf{k}_{0}=\mathbf{k}_{1}+\mathbf{k}_{2}
$$

onde $\mathbf{k}_{0}, \mathbf{k}_{1}$ e $\mathbf{k}_{2}$ são os vetores ${ }^{20}$ de onda do bombeio, sinal e complementar.

Existe duas possibilidades de satisfazer o acordo de fase: ou os fótons gêmeos produzidos possuem polarizações iguais (tipo 1) ou polarizações ortogonais (tipo 2), dependendo das características do cristal empregado na montagem. No nosso caso, o acordo é do tipo 2.

No OPO, todo o processo de conversão paramétrica acontece, como mencionando, em uma cavidade ótica que serve para selecionar os modos dos feixes sinal e complementar.

A depender das transmitâncias dos seus espelhos, essa cavidade pode ser ressonante somente para os gêmeos ou para os gêmeos e o bombeio também. Ser ressonante para um determinado feixe implica dizer que a cavidade possui uma alta finesse para esse feixe. Veremos a seguir que essa é a diferença básica na construção dos OPOs: DROPO1, DROPO2, TROPO1 e TROPO2.

Os feixes gerados, através da interação do bombeio com o cristal, são parcialmente refletidos pela cavidade e levam, como em um laser, a um processo de emissão estimulada.

Quando a taxa com que esse processo ocorre (ganho) é superior às perdas totais (perdas por transmissão mais perdas espúrias), produzem-se, acima de uma dada potência (limiar), os feixes gêmeos.

Se considerarmos o espectro de ruído de amplitude e fase dos feixes gêmeos, do ponto de vista teórico, veremos [5, 14] que ele é o resultado da dinâmica quântica estocástica dos campos ressonantes na cavidade.

Medir esse espectro, buscando por correlações intrinsecamente quânticas, emaranhamento, consiste no objetivo do trabalho experimental em Ótica Quântica. No nosso caso, em específico, não só medir emaranhamento como utilizá-lo em um protocolo de teletransporte.

Demonstra-se [14] que os espectros de ruído tanto de amplitude quanto de fase do sinal e do complementar apresentam excesso de ruído em ambas as quadraturas. A Figura 6.20 ilustra, esquematicamente, os estados quânticos dos gêmeos com excesso de ruído.

Uma outra maneira mais conveniente ${ }^{21}$ de considerar o estado dos gêmeos é fazer uma transformação no seu espaço de fase dadas pela soma e pela

\footnotetext{
${ }^{20} \mathrm{O}$ módulo do vetor de onda de cada campo no cristal é dado por $k_{i}=\frac{\omega_{i} n_{i}\left(\omega_{i}\right)}{c}$, onde $n_{i}\left(\omega_{i}\right)$ é o índice de refração do meio que depende da direção de propagação, polarização e frequência do feixe.

${ }^{21}$ Conveniente porque as quadraturas amplitude e fase dos gêmeos se desacoplam na matriz de covariância do OPO, não mencionada nesse texto [14].
} 

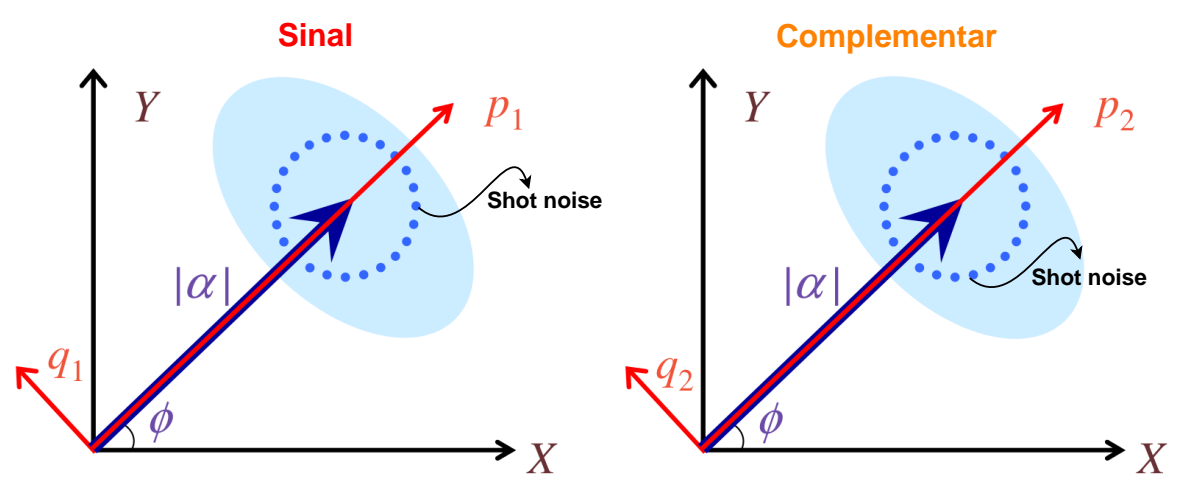

Figura 6.20: Figuras ilustrativas dos espaços de fase dos estados quânticos com excesso de ruído dos gêmeos produzidos no OPO. Quadraturas do sinal dadas por $\hat{p}_{1}$ e $\hat{q}_{1}$ e do complementar $\hat{p}_{2}$ e $\hat{q}_{2}$. O círculo pontilhado denota o estado coerente que possui o mínimo ruído quântico, que é usado como padrão ou escala na medida de outros ruídos. $|\alpha|$ é a intensidade da portadora e $\phi$ é a fase dela.

subtração de suas quadraturas, $\hat{p}_{ \pm}$e $\hat{q}_{ \pm}$:

$$
\hat{p}_{ \pm}=\frac{1}{\sqrt{2}}\left(\hat{p}_{1} \pm \hat{p}_{2}\right)
$$

e

$$
\hat{q}_{ \pm}=\frac{1}{\sqrt{2}}\left(\hat{q}_{1} \pm \hat{q}_{2}\right)
$$

A Figura 6.51 ilustra essa mudança no espaço de fase dos estados dos gêmeos.
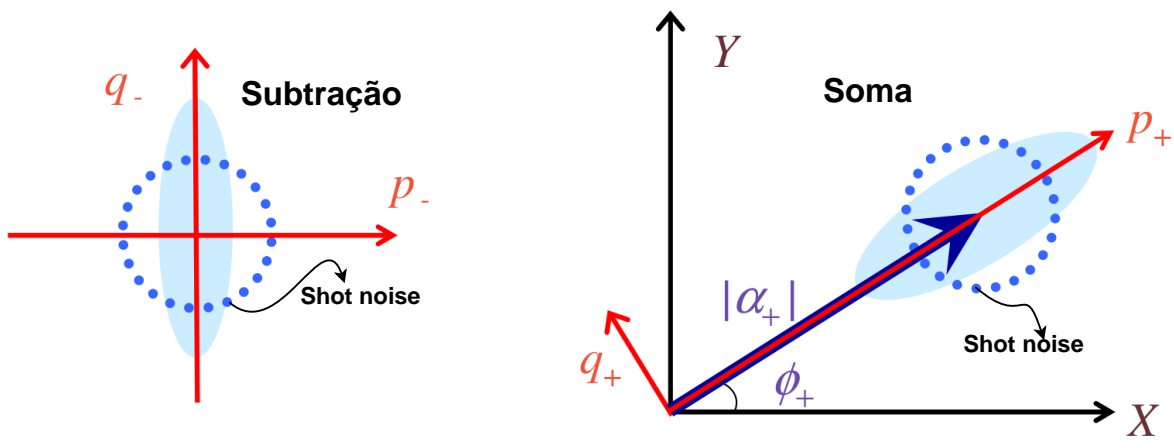

Figura 6.21: Figura ilustrativa das transformações no espaço de fase dos estados quânticos dos gêmeos produzidos no OPO. São visualizados a subtração e a soma. $|\alpha|$ é a intensidade da portadora e $\phi$ é a fase dela. 
É teoricamente conhecido $[5,14]$ que, para o OPO, a compressão de $\left\langle\Delta^{2} \hat{p}_{-}\right\rangle$e de $\left\langle\Delta^{2} \hat{q}_{-}\right\rangle$são funções da frequência de análise $\Omega$, a largura de banda da cavidade do OPO $(B W)$ e da relação entre as perdas (por transmissão e espúrias):

e

$$
\left\langle\Delta^{2} \hat{p}_{-}\right\rangle(\Omega)=1-\frac{A}{1+\left(\frac{\Omega}{B W}\right)^{2}},
$$

$$
\left\langle\Delta^{2} \hat{q}_{-}\right\rangle(\Omega)=1-\frac{A}{\left(\frac{\Omega}{B W}\right)^{2}}\left(1+\frac{\Delta^{2}}{1+\left(\frac{\Omega}{B W}\right)^{2}}\right),
$$

em que $\Delta$ é a dessintonia da cavidade e $A$ é igual a $\frac{\gamma}{\gamma^{\prime}}$, razão entre perdas por transmissão através do espelho 2 de acoplamento (figura 6.5) e perdas totais (transmissão mais espúrias). Estas curvas são normalizadas pelo ruído do shot noise e são visualizadas na Figura 6.22 para $A=1$, ou seja, no caso ideal, em que não há perdas e os gêmeos são perfeitamente ressonantes na cavidade $(\Delta=0)$.

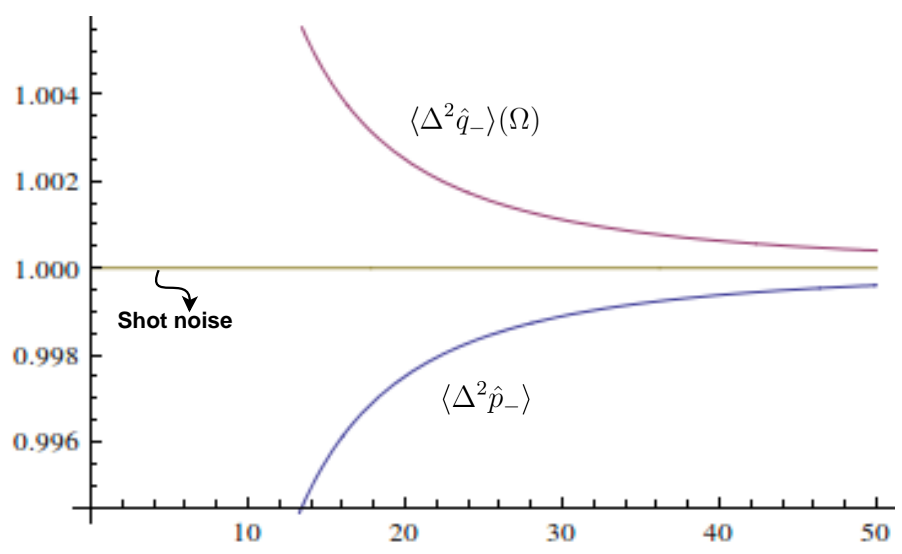

Figura 6.22: Previsão teórica das funções da compressão de ruído de intensidade, em azul, e compressão de ruído de fase, em rosa, ambas para $A=1$ e dessintonia igual a zero. A reta, em amarelo, representa o "shot noise".

Das equações (6.22) e (6.53), considerando $A=1$ e a dessintonia da cavidade nula, verificamos que os ruídos referente as quadraturas $\hat{p}_{-}$e $\hat{q}_{-}$é mínimo, considerando que o ruído do "shot noise" é igual a 1:

$$
\left\langle\Delta^{2} \hat{p}_{-}\right\rangle\left\langle\Delta^{2} \hat{q}_{-}\right\rangle=1
$$

Visto esse pequeno resumo da teoria conhecida do OPO pelo nosso grupo, passemos para os projetos e as tentativas de construção do canal quântico, onde se buscou a maior compressão de ruído de intensidade visando a máxima violação do critério DGCZ (5.1), no caso dos DROPOs, e a máxima violação do critério 5.3, no caso dos TROPOs. 


\subsubsection{Tentativa 1: DROPO1}

O projeto de construção de um OPO começa pela escolha do tipo de cristal que será empregado, pela determinação dos tipos de espelhos côncavos (determinado através dos seus raios de curvatura e coeficientes de transmissão para o verde e IR) usados na cavidade e pelo tamanho da cavidade, que deve levar em conta a questão geométrica.

No nosso caso, é uma exigência do protocolo de teletransporte que tenhamos uma compressão de ruído maior possível porque isso melhora a qualidade do emaranhamento produzido pelo OPO e, consequentemente, a fidelidade obtida no processo. A compressão de ruído $\left\langle\Delta^{2} \hat{p}_{-}\right\rangle$é, como visto anteriormente, altamente sensível às perdas ditas espúrias e também às perdas presentes na detecção.

Como mencionado no capítulo anterior, um OPO duplamente ressonante seria a fonte de estados emaranhados ideal para a implementação do teletransporte bicolor, dessa forma, as nossas duas primeiras tentativas foram com OPOs desse tipo. A primeira tentativa foi feita usando um cristal $^{22}$ KTP com uma das faces espelhada e coatings anti-refletores e está descrito, em detalhes, na dissertação [68] do estudante Igor Konieczniak, atualmente, estudante de doutorado do grupo e participante desse projeto.

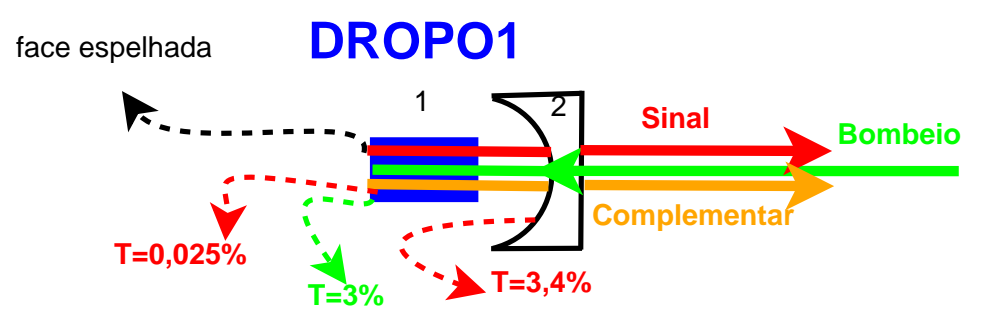

Figura 6.23: Figura ilustrativa da montagem do DROPO1. As transmitâncias em verde refere-se ao $532 \mathrm{~nm}$ e, em vermelho, ao $1064 \mathrm{~nm}$. O raio de curvatura do espelho, 2, côncavo é $50 \mathrm{~mm}$. Sobre o espelho é acoplado um PZT.

A Figura 6.23 ilustra o DROPO1 construído para o verde e o IR e os valores das transmitâncias do espelho utilizado e da face espelhada do cristal. O limiar de oscilação obtido foi em torno de $130 \mathrm{~mW}$ e a finesse $(F)$ para o IR medida com essa montagem foi de 133. Por meio desse valor, é possível facilmente inferir as perdas espúrias dentro da cavidade. Denotando-se por $P_{t o t}$ as perdas totais da cavidade, $T$ a transmissão e por $P_{e s p}$ as perdas espúrias,

\footnotetext{
${ }^{22}$ Cristal da marca Litton.
} 
temos que:

$$
P_{t o t}=T+P_{e s p}
$$

Assim, da relação conhecida para finesse, concluimos que:

$$
F=\frac{2 \pi}{T+P_{e s p}},
$$

Como visto na Figura 6.23, o espelho da montagem desse OPO tem uma transmissão de $3,4 \%$. Usando-se a equação (6.56) e o valor experimental da finesse, verificamos que $P_{e s p}=1,3 \%$, valor que inviabilizaria uma maior compressão de ruído dos gêmeos se comparado ao antigo TROPO do grupo ${ }^{23}$ [65].

Observando com mais cuidado as superfícies do cristal utilizado, percebemos que os "coatings" estavam aparentemente danificados. Uma explicação plausível para o valor das perdas espúrias ser maior nesse OPO talvez esteja relacionada a esse fato. Descartamos o uso desse cristal e partimos para a montagem do segundo DROPO.

\subsubsection{Tentativa 2: DROPO2}

Como a razão entre as perdas por transmissão e as perdas espúrias na cavidade afetam diretamente a compressão de ruído quântico e, consequentemente, a qualidade de emaranhamento entre os gêmeos, optamos por montar outro DROPO empregando, desta vez, outro cristal KTP usado em trabalhos anteriores do grupo $[2,4,6,60]$.

A Figura 6.24 exibe o esquema de montagem desse OPO com os valores das transmitâncias de ambas as faces dos espelhos côncavos envolvidos, assim como os seus raios de curvatura e comprimento de Rayleigh $z_{0}$. A Figura 6.25 ilustra o gráfico com a condição de ressonância de ambos os feixes usando os valores das transmitâncias da Figura 6.24 e a expressão teórica para a função de transmissão de uma cavidade ótica vista no capítulo 2.

Detalharemos o projeto do DROPO2, que buscou uma alta compressão de ruído de intensidade $\left\langle\Delta^{2} \hat{p}_{-}\right\rangle$para poder ser capaz de violar maximamente a desigualdade (5.1).

Da conhecida relação entre o "free spectral range" (FSR) e o tamanho ótico (L) de uma cavidade ótica:

$$
F S R=\frac{c}{2 L},
$$

\footnotetext{
${ }^{23} \mathrm{Na}$ época da montagem do DROPO1, existia na mesma mesa ótica um TROPO, descrito na tese [65], que estava sendo usado em outros trabalhos do grupo e esse apresentava mesma taxa de perda que o DROPO1. Como a ideia era superar o nível de compressão de ruído desse antigo OPO, resolvemos desconsiderar o DROPO1 como fonte de estados emaranhados.
} 


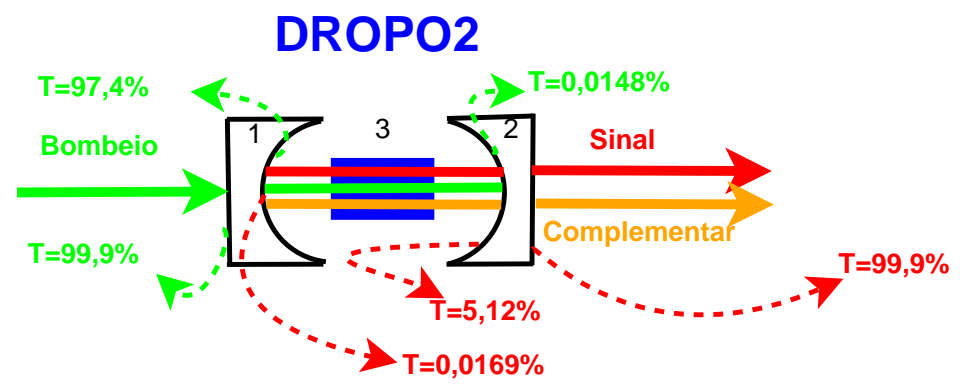

Figura 6.24: Configuração do DROPO2 montado no interior da câmara de vácuo. São vistas as transmitâncias para o verde e o IR dos espelhos côncavos 1 e 2 da cavidade montada para abrigar o cristal KTP, 3. Raio de curvatura de ambos espelhos é igual a 10mm. Sobre o espelho 2 é acoplado um PZT. Comprimento de Rayleigh $\left(z_{0}\right)$ igual a $3,57 \mathrm{~mm}$.

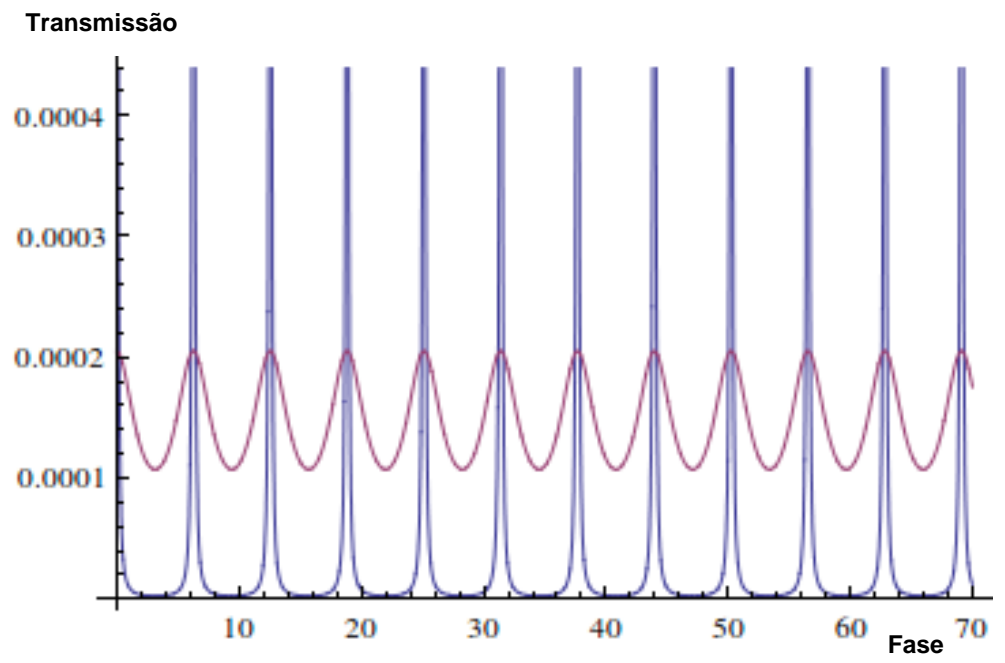

Figura 6.25: Picos de transmissão em função da fase para o 532nm, em rosa, e para o $1064 \mathrm{~nm}$, em azul, do DROPO2, ilustrando a condição de ressonância dos feixes no interior da cavidade. A fase dos feixes é variada através da mudança do comprimento da cavidade, que é realizada pelo PZT. 
projetamos o tamanho da cavidade do DROPO2, de forma que ela fornecesse um $F S R=5 G H z$. Teoricamente, considerando-se as perdas por transmissão para o feixe de $1064 \mathrm{~nm}$, vistos na Figura 6.5, e perdas espúrias estimadas em $0,5 \%$, temos uma finesse (F) igual a 111. Assim, com os valores da finesse e o FSR, obtemos uma largura de banda (BW):

$$
B W=\frac{F S R}{F}=45 M H z .
$$

Já a razão entre as perdas $\frac{\gamma}{\gamma^{\prime}}$, com a estimativa de perdas espúrias de $0,5 \%$, é igual a 0,9. Substituindo-se na equação (6.22) esse valor juntamente com o valor (6.58) para o BW e considerando-se, inicialmente, uma frequência de análise igual a $18 \mathrm{MHz}$, resulta em uma compressão $\left\langle\Delta^{2} \hat{p}_{-}\right\rangle=-6,1 d B$.

Obviamente, esse é apenas um valor estimado e não leva em conta outros tipos de perdas como perdas em elementos óticos, externos ao DROPO2, usado para detectar os feixes, e perdas devido à detecção. Todas essas perdas levam a uma redução da compressão de ruído $\left\langle\Delta^{2} \hat{p}_{-}\right\rangle$.

Feito o acordo de modo do feixe de bombeio, vindo diretamente do Diabolo, com o DROPO2, obtemos um limiar de oscilação em torno de $450-500 \mathrm{~mW}$, a depender das perdas espúrias contidas nos espelhos da cavidade e no cristal. Em geral, em condições otimizadas, as perdas espúrias ficavam em torno de $0,7 \%$.

Para a realização das medidas de ruído quântico, é necessário travar a cavidade na frequência dos gêmeos produzidos para que se intensifique o efeito da conversão paramétrica, além de selecionar o modo dos campos que serão futuramente medidos.

Dessa forma, empregamos o método Pound-Drever-Hall [69] (PDH) para travar a cavidade do DROPO2, usando a modulação de $12 \mathrm{MHz}$ fornecida pelo laser. Em caráter ilustrativo, são vistos nas Figuras 6.26 e 6.27 um exemplo típico de uma condição de oscilação e de travamento.

\section{Primeiras medidas de compressão de ruído quântico}

Após a montagem e travamento do OPO, verificamos a linearidade do sistema eletrônico de aquisição via LabView através da calibração do "shot noise" e, só então, partimos para as medidas de compressão de ruído.

Inicialmente, para facilitar, focamos nossa análise somente no ruído da quadratura de intensidade $\left\langle\Delta^{2} \hat{p}_{-}\right\rangle$(subtração) e $\left\langle\Delta^{2} \hat{p}_{+}\right\rangle$(soma), abandonando temporariamente as cavidades de análise usadas na detecção autohomodina dos feixes.

Com essa medida preliminar da compressão de $\left\langle\Delta^{2} \hat{p}_{-}\right\rangle$e, comparando com resultados anteriores obtidos pelo grupo [14, 70, 71, 65, 72], é possível 

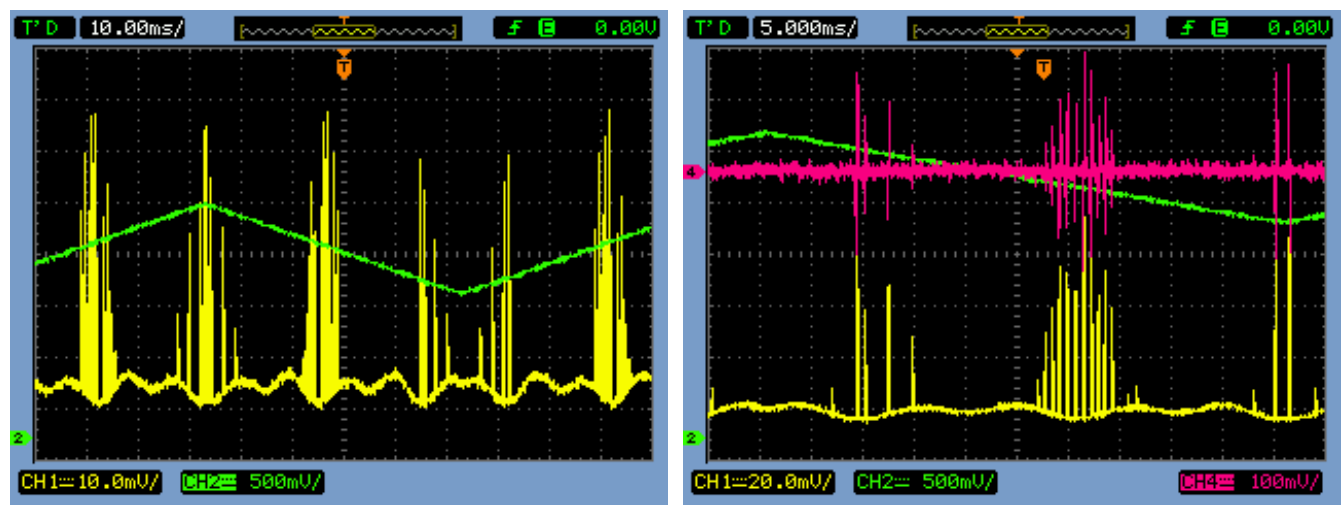

Figura 6.26: Figuras meramente ilustrativas das imagens vistas no osciloscópio dos picos de transmissão dos feixes gêmeos produzidos pelo DROPO2. Picos em amarelo, varredura do sinal enviado ao PZT, acoplado ao espelho 2, em verde. Em rosa, pode ser visto o sinal de erro usado no travamento do OPO.
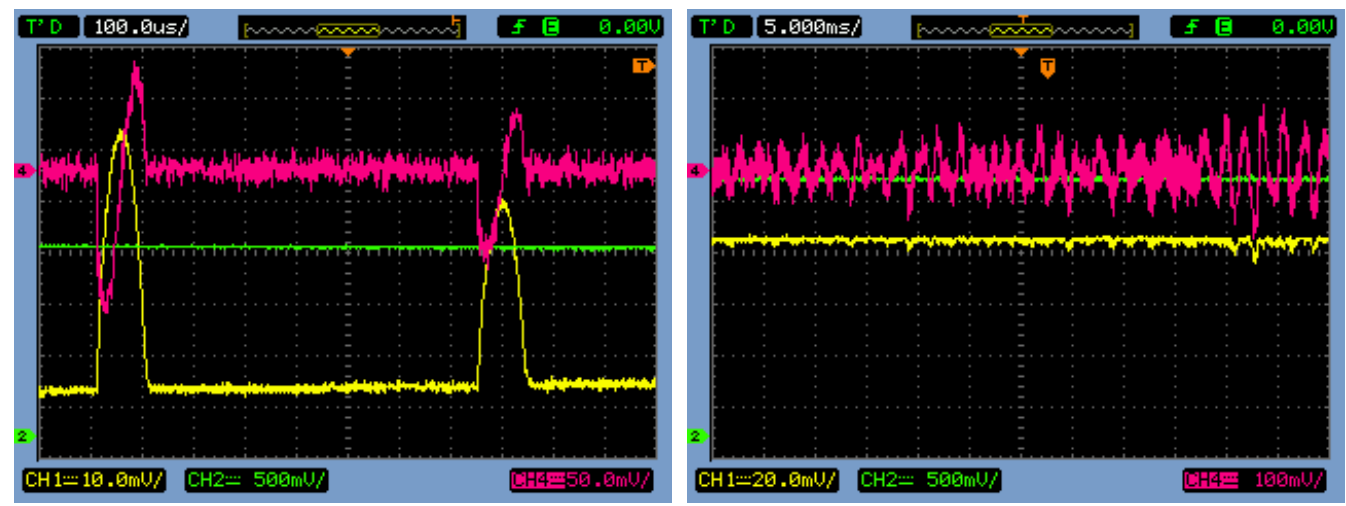

Figura 6.27: Figuras meramente ilustrativas das imagens vistas no osciloscópio, em uma escala temporal menor, mostrando em detalhe os picos de transmissão e respectivo sinal de erro. À direita, uma situação típica de travamento. 
ter uma idéia do emaranhamento produzido pelo DROPO2 e, assim, alinhar as cavidades de análise para a realização completa das medidas de ruído de intensidade $\left(\left\langle\Delta^{2} \hat{p}_{-}\right\rangle\right)$e fase $\left(\left\langle\Delta^{2} \hat{q}_{+}\right\rangle\right)$.

Como visto no gráfico 6.22 , a compressão, em relação ao "shot noise", torna-se cada vez maior à medida que diminui-se a frequência de análise. Entretanto, em termos práticos, é difícil fazer medidas em baixas frequências em virtude da própria limitação do aparato de medida, que introduz um ruído eletrônico às medidas.

Tendo em vista essa limitação, a largura de banda de nossas cavidades de análise e a frequência de modulação de fase do nosso laser comercial Diabolo optamos por usar inicialmente uma frequência de análise $18 \mathrm{MHz}$ e, posteriormente, nas medidas realizadas com as cavidades de análise mudamos para $15 M H z$.

A fim de medirmos $\left\langle\Delta^{2} \hat{p}_{-}\right\rangle$em intensidade, montamos o esquema experimental visto na figura 6.28. Nesse esquema, os feixes sinal (denotado por 1) e complementar (denotado por 2) que deixam a cavidade, incidem em um PBS, que os divide de acordo com a polarização de cada um. Cada feixe gêmeo é, então, direcionado para sua respectiva detecção balanceada.

Denota-se por $H F 1.1$ e HF1.2 os sinais em alta frequência dos detectores provenientes do feixe gêmeo sinal, das partes transmitida e refletida pelo PBS. Para o gêmeo complementar, os mesmos são denotados por HF2.1 e HF2.2.

Os sinais HF de cada detector passam por uma cadeia de demodulação, e, os sinais resultantes de todo o processo são recebidos pela placa de aquisição do National Instruments, conectada ao LabView, que converte os sinais analógicos em digitais para que sejam processados pelo computador. Cada aquisição de medida possui 10.000 pontos e é feita balanceadando-se a potência em ambos os detectores responsáveis pela detecção de cada feixe gêmeo. Esse balanceio é realizado ajustando-se as lâminas de meia onda de forma a igualar as potências e observando-se os sinais DC de todos os quatro detectores envolvidos

A fim de descontar o ruído eletrônico, ao final de cada medida, fazemos uma aquisição sem incidir luz nos detectores. Na tabela A.1, pode-se ver um exemplo típico de valores do ruído eletrônico produzido no nosso sistema de medição, que introduz um erro sistemático nas nossas medidas de ruído quântico, caso não seja levado em consideração. Esses valores, claramente, dependem de toda a complexa cadeia de tratamento eletrônico dos sinais HFs vindos dos detectores. Todas as medidas de ruído apresentadas ao longo desse texto são expressas considerando

Na tabela, mostra-se os valores da variância de cada sinal temporal, vindo da cadeia de demodulação, e recebido por cada canal da placa de aquisição. Em um abuso de linguagem, usou-se HF1(ou2).1(ou2) somente para indi- 


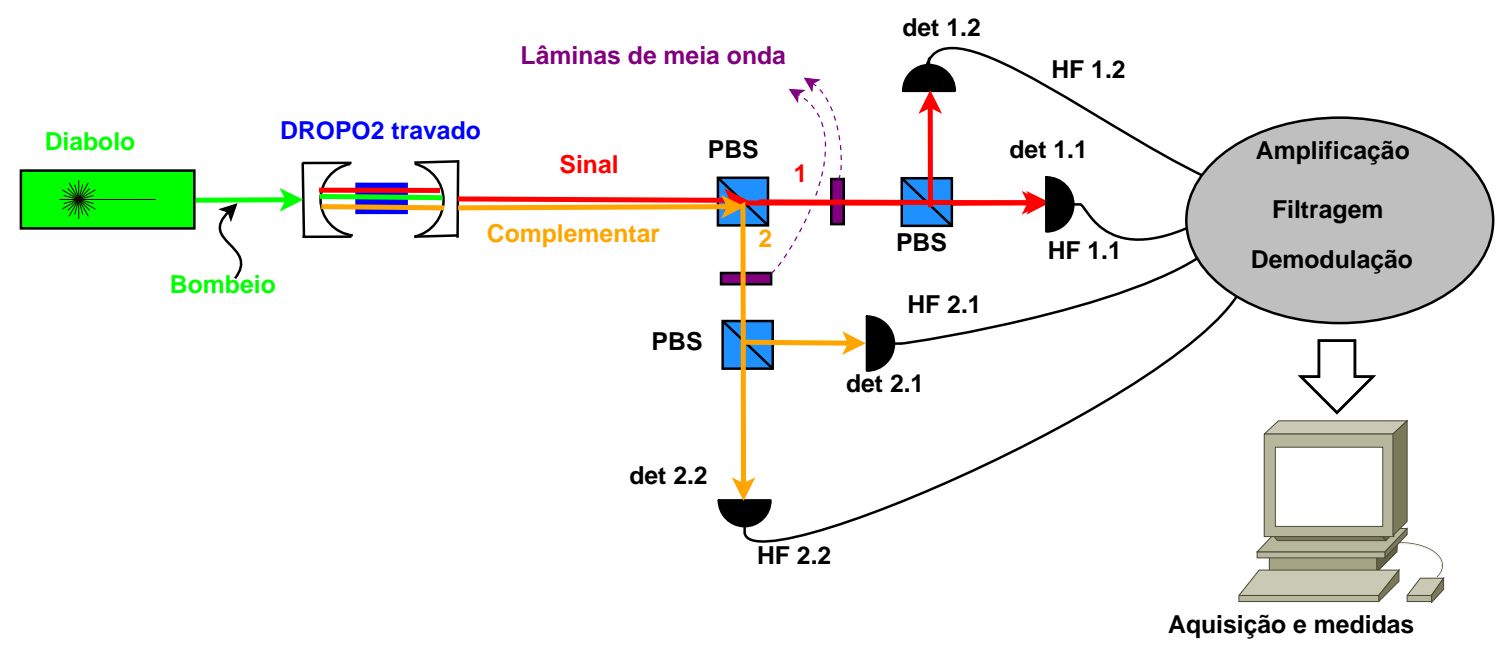

Figura 6.28: Padrão de cores adotados para os elementos óticos: retângulo verde (isolador ótico), cubo azul (PBS) e retângulo roxo (lâmina de meia onda). Esquema experimental de medida de compressão de ruído de intensidade $\left\langle\Delta^{2} \hat{p}_{-}\right\rangle$(subtração). Nessas medidas, o OPO era travado e o sistema de aquisição acionado através do computador.Sinais DCs omitidos na figura.

\begin{tabular}{|l|l|}
\hline Ruído eletrônico & Volts \\
\hline $\operatorname{Var}(H F 1.1)$ & 0.0016 \\
\hline $\operatorname{Var}(H F 1.2)$ & 0.0014 \\
\hline $\operatorname{Var}(H F 2.1)$ & 0.0025 \\
\hline $\operatorname{Var}(H F 2.2)$ & 0.0015 \\
\hline Soma das variâncias & 0.0070 \\
\hline
\end{tabular}

Tabela 6.1: Tabela ilustrando o ruído eletrônico típico do nosso sistema de aquisição via LabView. Cada sinal HF demodulado recebido pela placa de aquisição é um processo estocástico de 10.000 medidas. Os valores vistos acima dizem respeito às variâncias de cada um desses processos. Na medição do ruído proveniente da natureza quântica da luz, faz-se necessário descontar esse ruído sistemático.

car a origem do sinal inicial (qual detector), porém, na verdade, após a demodulação, sabe-se que esses sinais deixam de ser $H F$ e passam a conter frequências máximas em tono de $300 \mathrm{~K} \mathrm{~Hz}$.

Inúmeros testes e modificações eletrônicas foram realizadas, especialmente para resolver o problema na medida de calibração do "shot noise". Resolvido o problema da não-linearidade eletrônica, que nos impedia de medir o 
"shot noise" corretamente, partimos para a medida de compressão de ruído de intensidade propriamente dita.

As medidas de $\left\langle\Delta^{2} \hat{p}_{-}\right\rangle$(subtração) e $\left\langle\Delta^{2} \hat{p}_{+}\right\rangle$(soma) foram feitas mediante o tratamento estatístico dos processos estocásticos produzidos. Assim, a medida da subtração é a variância da série temporal:

$$
[(H F 1.1+H . F 1.2)-(H F 2.1+H . F 2.2)],
$$

e a medida da soma é a variância da série:

$$
[(H F 1.1+H . F 1.2)+(H F 2.1+H . F 2.2)] .
$$

Ambas as medidas são calibradas em relação ao "shot noise", considerado com a variância da série:

$$
[(H F 1.1-H . F 1.2) \pm(H F 2.1-H . F 2.2)] .
$$

A expressão para o "shot noise" da equação (6.61) indica que ele pode ser calculado de duas formas. Em uma condição de perfeita calibração, essas expressões são iguais, conforme verificaremos nas medidas da tabela 6.32 .

As Figuras 6.29 e 6.30 ilustram os processos estocásticos resultantes da aquisição da medida do esquema da Figura 6.28. Em ambas as figuras, é visto o nível de ruído do "shot noise" que é comparados aos outros níveis de ruído, o da subtração e o da soma. No da subtração, vemos uma compressão do nível de ruído em relação ao "shot noise" e, na soma, um excesso de ruído.

A Figura 6.31 ilustra os sinais DCs dos quatro detectores da montagem 6.28, diretamente recebidos pelo LabView, durante a realização da medidas referente as Figuras 6.29 e 6.30. O controle desses sinais DCs é de fundamental importância para garantir que cada par de detectores esteja balanceado. Como vimos na seção 6.1, o sinal DC informa, através da sua calibração, o quanto de potência incide no detector.

As oscilações observadas dentro da janela de aquisição temporal são resultados da própria qualidade do travamento da cavidade do DROPO2. Ruídos sonoros ou vibrações mecânicas, por exemplo, tornam o travamento da cavidade mais difícil e os sinais DCs mais ruidosos.

A tabela da Figura 6.32 ilustra resumidamente o melhor resultado obtido para compressão de ruído do DROPO2. Na figura 6.32, podem ser vistos, além da variância do sinal recebido por cada canal, o esquema usado para medir as correlações entre os feixes gêmeos, ou seja, os ruídos de soma e subtração. De posse desses valores, é possível, então, calcular o valor da compressão em $d B$.

Ainda na tabela 6.32, em destaque, em vermelho, é visto que os valores para o "shot noise" são iguais, como deveriam ser, pois, a variância da 


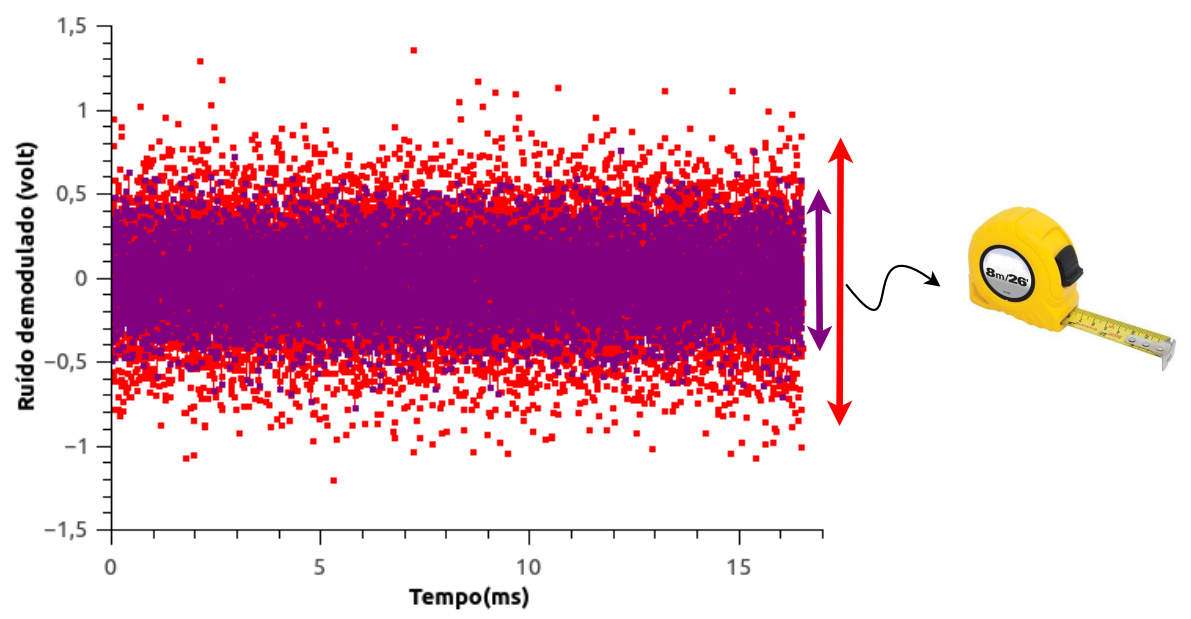

Figura 6.29: Séries temporais de 10.000 pontos das medidas referente a tabela A.1 realizadas em uma frequência de análise de $18 \mathrm{MHz}$. Em roxo, a subtração $[(H F 1.1+H . F 1.2)-(H F 2.1+H . F 2.2)]$ e, em vermelho, o "shot noise" [(HF1.1-H.F1.2) - (HF2.1-H.F2.2)]. Comparando-se a subtração com o "shot noise", percebemos o caráter de compressão de $\left\langle\Delta^{2} \hat{p}_{-}\right\rangle$.

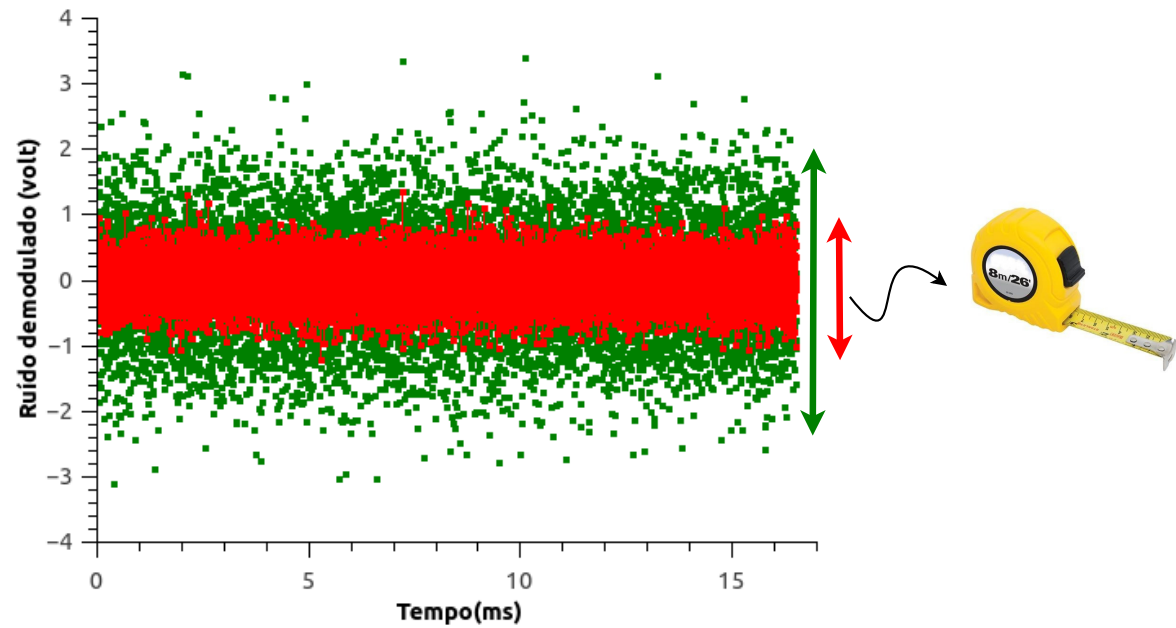

Figura 6.30: Séries temporais de 10.000 pontos das medidas referente a tabela A.1 realizadas em uma frequência de análise de $18 M H z$. Em verde, a soma $[(H F 1.1+H . F 1.2)+(H F 2.1+H . F 2.2)] \mathrm{e}$, em vermelho, o "shot noise" $[(H F 1.1-H . F 1.2)-(H F 2.1-H . F 2.2)]$. Comparando-se a soma com o "shot noise", percebemos o caráter de excesso de ruído de $\left\langle\Delta^{2} \hat{p}_{+}\right\rangle$. 


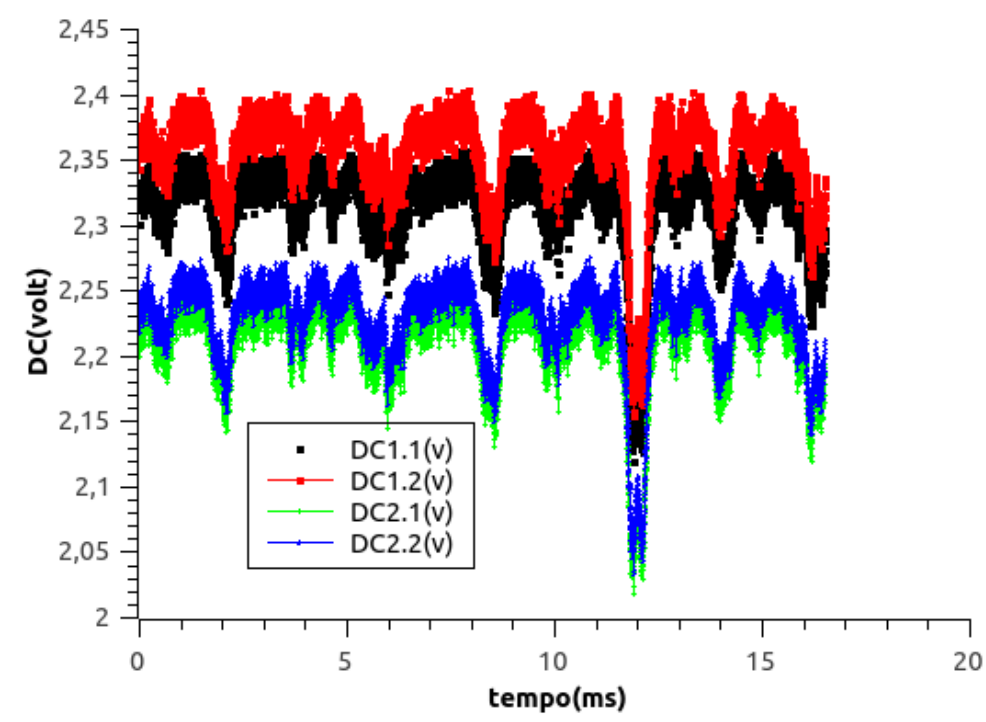

Figura 6.31: Sinais DCs dos quatro detectores da montagem 6.28 diretamente recebidos pela placa de aquisição do Lab View durante a realização da medida referente a tabela 6.32 .

\begin{tabular}{|c|c|}
\hline Variância(HF1.1 ) & $\mathbf{0 , 0 5 6}$ \\
\hline Variância(HF1.2 ) & $\mathbf{0 , 0 5 2}$ \\
\hline Variância(HF2.1) & $\mathbf{0 , 0 7 9}$ \\
\hline Variância(HF2.2) & $\mathbf{0 , 0 5 1}$ \\
\hline Shot noise- Var[(HF1.1-HF1.2) + (HF2.1-HF2.2)] & 0,106 \\
\hline Shot noise- Var[(HF1.1-HF1.2) - (HF2.1-HF2.2)] & 0,103 \\
\hline & \\
\hline Ruído da soma dos feixes- Var[(HF1.1+HF1.2) + (HF2.1+HF2.2)] & $\mathbf{0 , 6 9 8}$ \\
\hline Ruído da subtração dos feixes- Var[(HF1.1+HF1.2) - (HF2.1+HF2.2)] & $\mathbf{0 , 0 4 2}$ \\
\hline Descontando o ruido eletrônico & \\
\hline Ruído da soma dos feixes- Var[(HF1.1+HF1.2) + (HF2.1+HF2.2)] & $\mathbf{0 , 6 9 1}$ \\
\hline Ruído da subtração dos feixes- Var[(HF1.1+HF1.2) - (HF2.1+HF2.2)] & $\mathbf{0 , 0 3 5}$ \\
\hline Shot noise- Var[(HF1.1-HF1.2) + (HF2.1-HF2.2)] & $\mathbf{0 , 0 9 9}$ \\
\hline Shot noise- Var[(HF1.1-HF1.2) - (HF2.1-HF2.2)] & $\mathbf{0 , 0 9 6}$ \\
\hline Compressão de ruído normalizado pelo shot noise & \\
\hline Var[(HF1.1+HF1.2) - (HF2.1+HF2.2)] & $-4,38 \mathrm{~dB}$ \\
\hline
\end{tabular}

Figura 6.32: Tabela com o melhor resultado obtido ao medir compressão de ruído de intensidade do DROPO2. Como na tabela anterior, os valores acima referem-se às variâncias de cada uma das séries temporais de 10.000 medidas adquiridas via LabView realizadas em uma frequência de análise de $18 \mathrm{M} \mathrm{Hz}$. Unidade: Volt 
soma de duas variáveis aleatórias independentes (sem correlações) é igual à variância da subtração das mesmas. Valores iguais para as duas formas de cálculo do "shot noise" indicam, conforme já dito, que nosso sistema de medida é confiável.

Esse resultado de $-4,38 d B$ apresentou o maior nível de compressão de ruído quântico já medido na história do nosso laboratório. Entretanto, apesar de muito "animador" em um primeiro momento, ainda não sabíamos nada sobre a medida do ruído de fase $\left\langle\Delta^{2} \hat{q}_{+}\right\rangle$que nos diria, afinal, se a desigualdade de Duan (5.1) foi violada e se o DROPO2 poderia ser o canal quântico da nossa proposta inicial para o teletransporte bicolor, vista na Figura 5.4.

Como discutido no capítulo 3 , a medida do ruído de fase é realizada somente mediante o uso de técnicas interferométricas como a detecção homodina e a auto-homodina [8], que tornam possível a medida do ruído de fase através de sua projeção em ruído de intensidade. No caso da detecção auto-homodina, a cavidade de análise, ao ser varrida, é a responsável pela rotação da elipse de ruído do feixe [8].

Assim, visando as medidas de ambas as quadraturas, alinhamos e fizemos o acordo de modo dos gêmeos com as duas cavidades de análise. A ideia é detectar os feixes refletidos por essas cavidades e enviá-los para a sua respectiva unidade de detecção balanceada para a medida.

\section{Medidas realizadas com as cavidades óticas de análise}

Para medirmos $\left\langle\Delta^{2} \hat{p}_{-}\right\rangle$e $\left\langle\Delta^{2} \hat{q}_{+}\right\rangle$, montamos o esquema experimental visto na Figura 6.33. Os feixes sinal (1) e complementar (2) incidem em um PBS, que os divide de acordo com a polarização de cada um. Cada feixe gêmeo é, então, direcionado para sua respectiva cavidade de análise e, ao ser refletido, ele é levado para a sua detecção balanceada. A Figura 6.34 exibe uma fotografia da montagem do esquema 6.33 na mesa ótica.

Durante a realização da medida, o cristal KTP tem sua temperatura estabilizada por um circuito eletrônico e os PZTs das cavidades de análise recebem um sinal de varredura do tipo triangular. Além disso, o DROPO2 é travado na ressonância do IR e o sistema de aquisição via Lab View é acionado.

Uma importante condição a ser obedecida durante a realização da medida é garantir que ambas as cavidades de análise entrem em ressonância ao mesmo tempo para poder garantir que as elipses de ruído dos feixes gêmeos giram em sincronia. Para isso, monitora-se os sinais de transmissão de ambas as cavidades de análise, controlando as suas varreduras de forma que os picos de transmissão das duas cavidades se sobreponham. Somente nesse instante, a medida é adquirida. A Figura 6.36 ilustra a condição de transmissão de ambas as cavidades de análise no momento da medida referente a Figura 


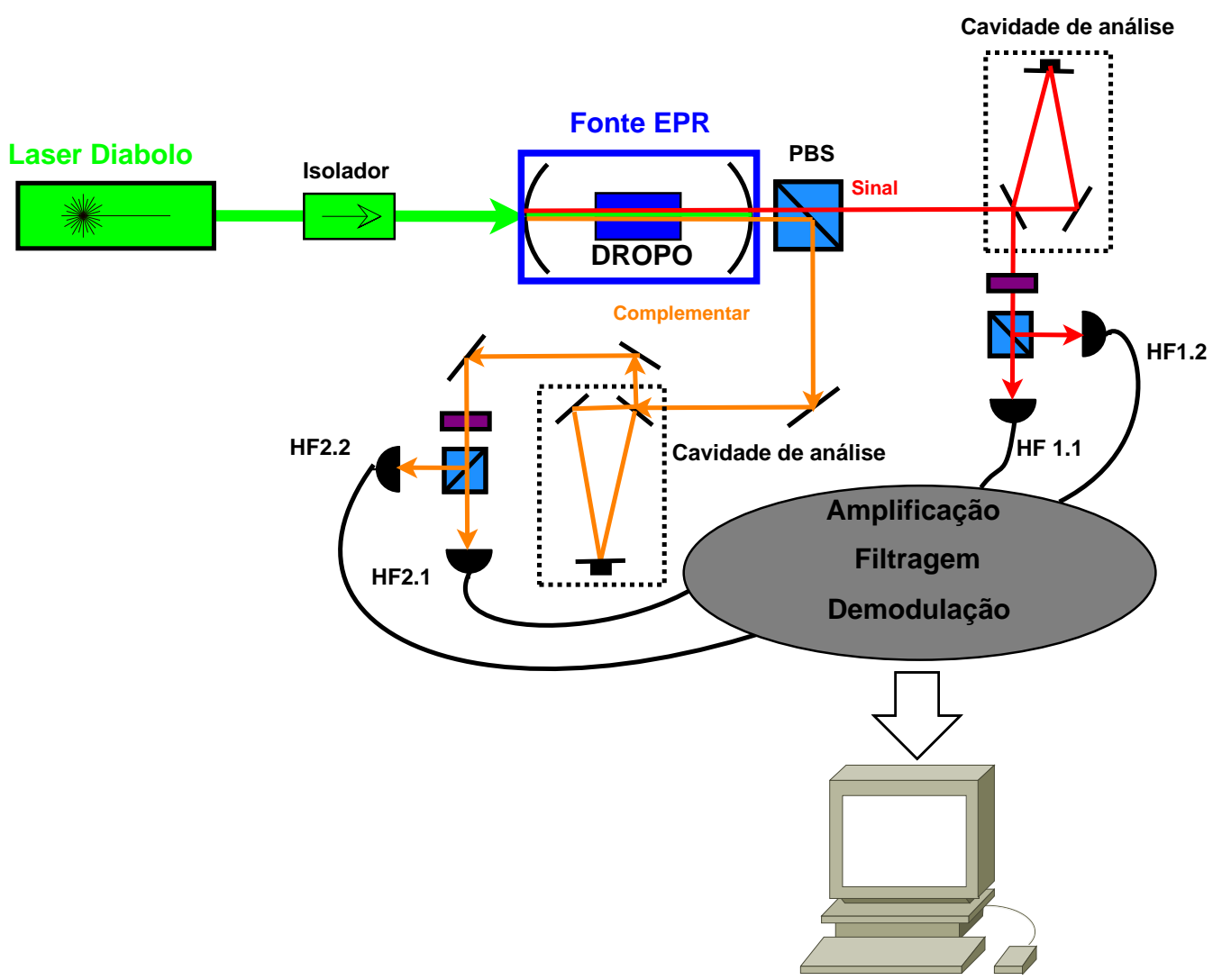

Figura 6.33: Padrão de cores adotados para os elementos óticos: retângulo verde (isolador ótico), cubo azul (PBS) e retângulo roxo (lâmina de meia onda). Esquema experimental para a realização das medidas $\left\langle\Delta^{2} \hat{p}_{-}\right\rangle$(subtração) e $\left\langle\Delta^{2} \hat{q}_{+}\right\rangle$(soma). Nessas medidas, o OPO era travado e as duas cavidades de análise eram varridas simultaneamente. Sinais DC omitidos na figura. 


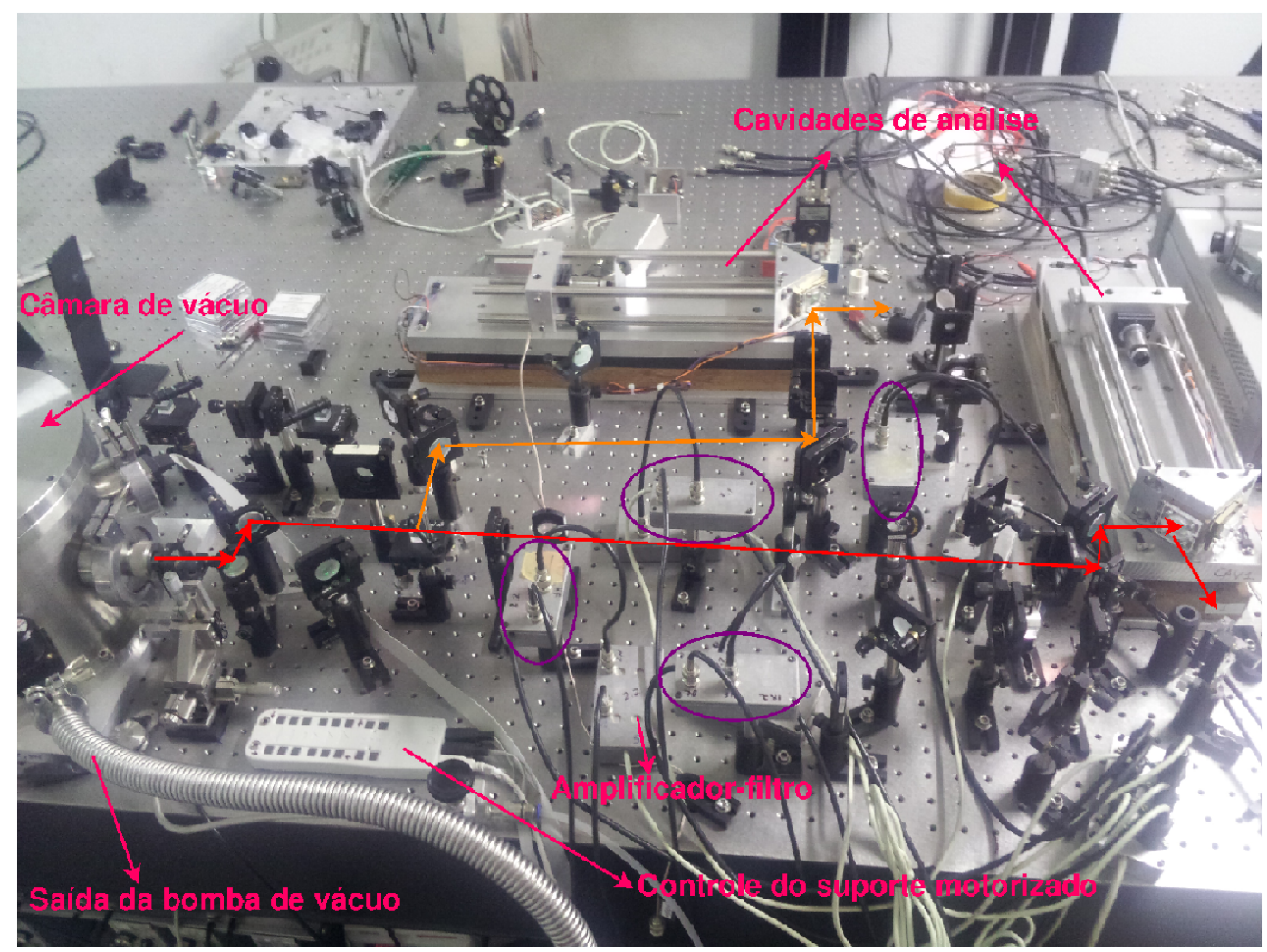

Figura 6.34: Vista de cima da montagem geral usada na realização das medidas com o DROPO2. À esquerda, é visualizada a câmara de vácuo que abriga o DROPO2 e de onde partem os gêmeos e a saída da bomba de vácuo. Logo após a câmara, há um PBS que separa os gêmeos de acordo com sua polarização. Os caminhos óticos dos gêmeos estão destacados em vermelho e laranja. Cada gêmeo é direcionado à uma cavidade de análise para medição de suas quadraturas usando a técnica da detecção auto-homodina. Os gêmeos são refletidos pelas cavidades de análise e são dirigidos aos núcleos "lâmina de meia onda+PBS+detectores" para a realização da medida. Em roxo, são vistos os quatro detectores envolvidos nas medidas. Também pode ser visualizado um dos quatro amplificador-filtro usado nas medidas. 
6.37.

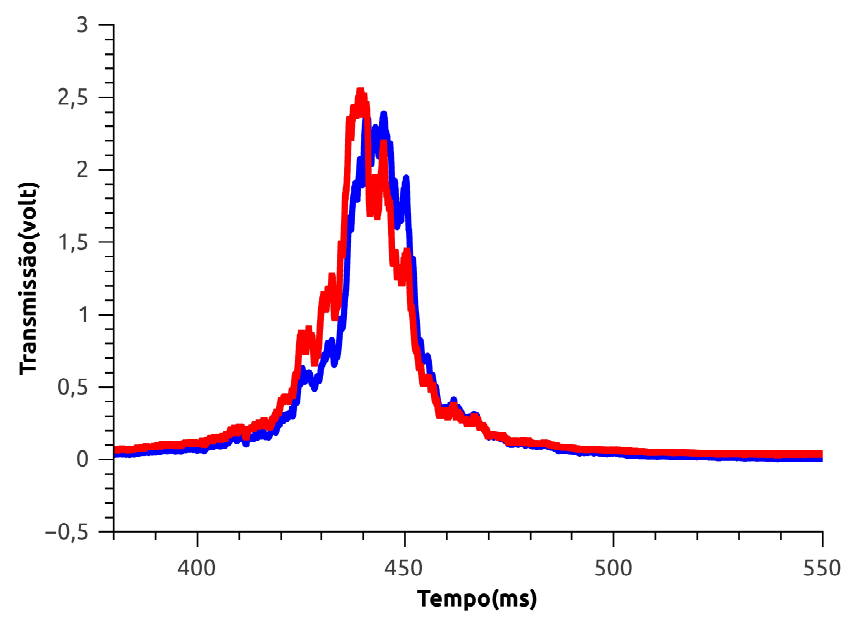

Figura 6.35: Sinais de transmissão, adquiridos via Lab View, das duas cavidades de análise no momento da realização da medida referente a Figura 6.37. A condição que elas estejam em ressonância no exato momento da aquisição da medida é fundamental para garantir que as elipses de ruído dos gêmeos estejam girando em sincronia.

Inicialmente, a primeira bateria de medidas realizadas com essa montagem foi feita com o cristal KTP a uma temperatura ${ }^{24}$ de $18^{\circ} \mathrm{C}$. Depois, com o propósito de reduzir o excesso de ruído de fase adquirido por meio da interação entre os fônons da rede cristalina e os fótons, diminuimos a temperatura do cristal acionando o Peltier da montagem descrita na seção 6.2.

A análise das séries temporais adquiridas após as medidas, usando as cavidades de análise, é a mesma vista na equação 6.59 para subtração, na equação 6.60 para soma e na equação 6.61 para o "shot noise". Entretanto, como a cavidade de análise é varrida no tempo, o tratamento das curvas resultantes da subtração e soma é diferente. Da curva da subtração, extraimos a informação de $\left\langle\Delta^{2} \hat{p}_{-}\right\rangle$e, da curva da soma, a informação de $\left\langle\Delta^{2} \hat{q}_{+}\right\rangle$ no momento que a elipse de ruído é girada e, assim, a informação de fase é projetada em intensidade.

Cada aquisição de medida contém 450.000 pontos dentro de uma janela temporal de $740 \mathrm{~ms}$ e representa um processo estocástico. Como experimentalmente a medida de ruído é obtida em função do tempo, é necessário fazer

\footnotetext{
${ }^{24}$ Temperatura da água circulante na base estabilizada pelo Chiller.
} 


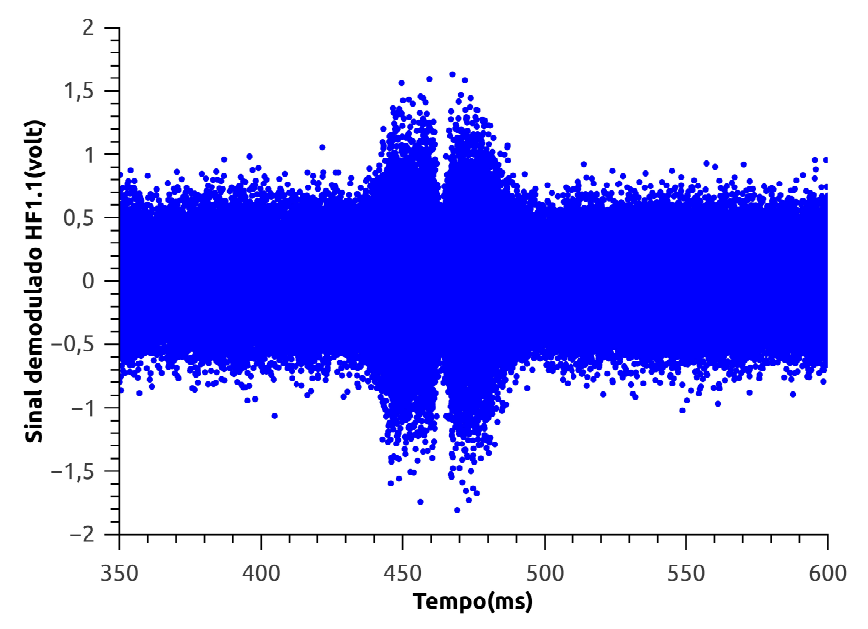

Figura 6.36: Exemplo do sinal demodulado proveniente diretamente do detector 1.1 e adquirido através da placa de aquisição do LabView. Observa-se excesso de ruído na série temporal no momento em que a cavidade de análise entra em ressonância. Esse sinal juntamente com os outros sinais HF1.2, HF2.1 e HF2.2 são tratados estatisticamente para fornecer as medidas de $\left\langle\Delta^{2} \hat{p}_{-}\right\rangle$e $\left\langle\Delta^{2} \hat{q}_{+}\right\rangle$.

uma transformação para fazê-la como função da dessintonia da cavidade de análise.

Para cada instante de tempo $(t)$ em que a cavidade é varrida, a dessintonia $(\Delta)$ é calculada levando-se em consideração o tempo onde ocorre o pico de transmissão da cavidade ( $t_{\text {trans }}$ ) e a largura de banda do pico, fornecida em termos temporais $\left(B W_{\text {temporal }}\right)$ :

$$
\Delta=\frac{\left(t-t_{\text {trans }}\right)}{B W_{\text {temporal }}}
$$

A variância é calculada de 1.000 em 1.000 pontos e a curva obtida é, então, ajustada à curva teórica, vista no capítulo 3 , para o espectro de ruído (do campo refletido pela cavidade) e as informações de $\left\langle\Delta^{2} \hat{p}_{-}\right\rangle$e $\left\langle\Delta^{2} \hat{q}_{+}\right\rangle$diretamente extraídas do ajuste.

As Figuras 6.37, 6.38 e 6.39 exibem as curvas resultantes da análise das medidas de ruído com o cristal $\mathrm{KTP}$ a $18^{\circ} \mathrm{C}, 0^{\circ} \mathrm{C}$ e $-4^{\circ} \mathrm{C}$ de temperatura respectivamente.

A tabela 6.2 resume as medidas obtidas para $\left\langle\Delta^{2} \hat{p}_{-}\right\rangle$e $\left\langle\Delta^{2} \hat{q}_{+}\right\rangle$nas três temperaturas a que o cristal foi submetido. Em todas elas, o excesso de ruído da soma é muito alto e impossibilita a violação do critério DGCZ. A 


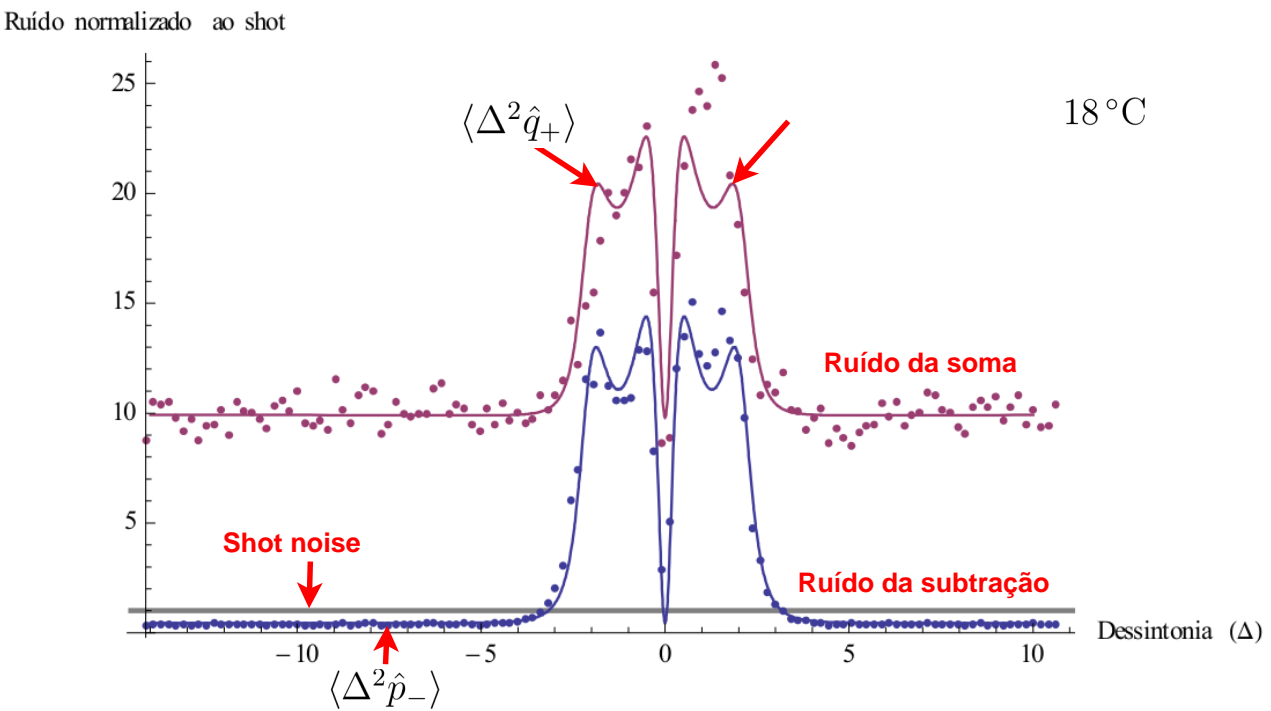

Figura 6.37: Medida linear em função da dessintonia da cavidade, calibrada pelo "shot noise", do ruído quântico do DROPO2 a $18^{\circ} \mathrm{C}$ apresentando um excesso de ruído de $\left\langle\Delta^{2} \hat{q}_{+}\right\rangle$que impediu a violação do critério DGCZ.

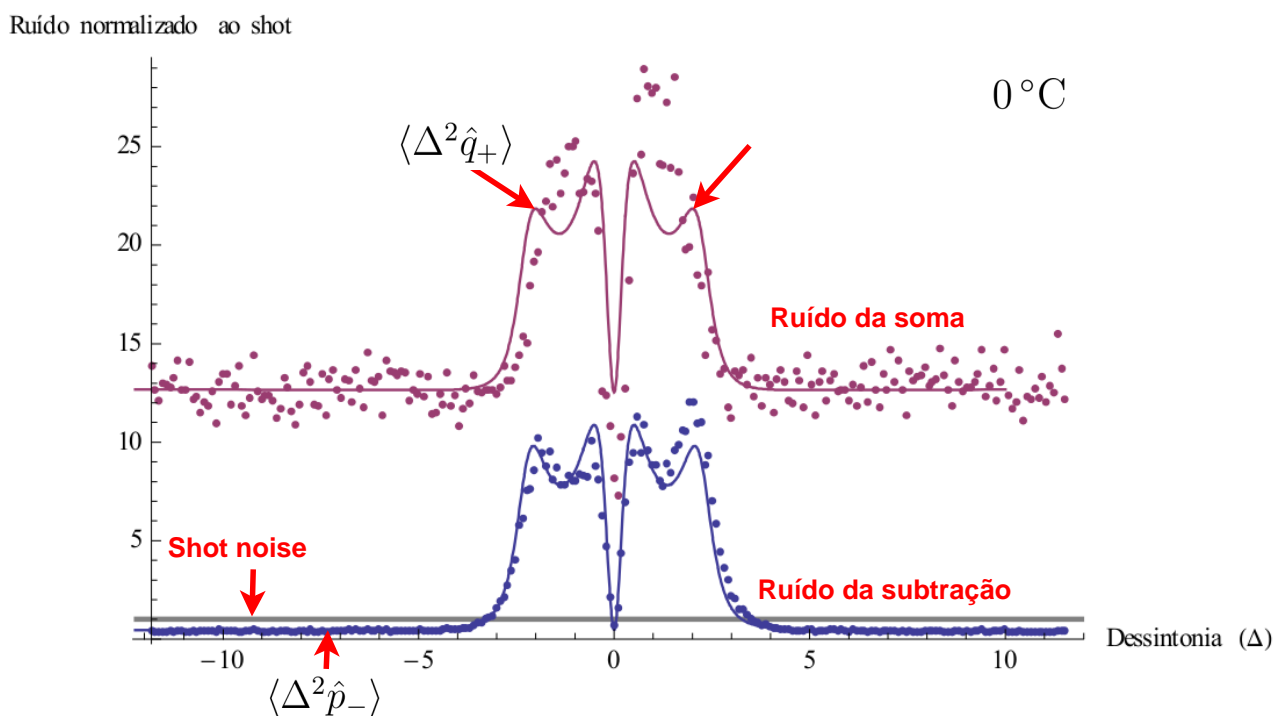

Figura 6.38: Medida linear em função da dessintonia da cavidade, calibrada pelo "shot noise", do ruído quântico do DROPO2 a $0{ }^{\circ} \mathrm{C}$ apresentando um excesso de ruído de $\left\langle\Delta^{2} \hat{q}_{+}\right\rangle$que impediu a violação do critério DGCZ. 


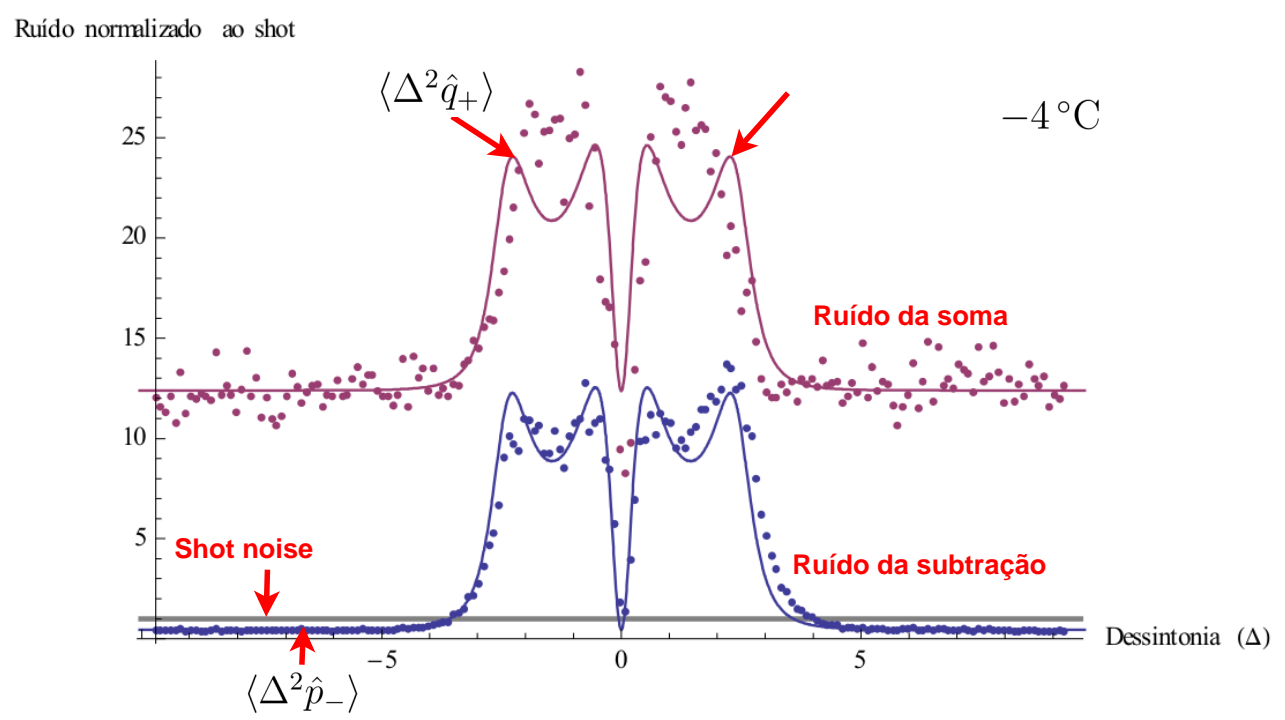

Figura 6.39: Medida linear em função da dessintonia da cavidade, calibrada pelo "shot noise", do ruído quântico do DROPO2 a $-4{ }^{\circ} \mathrm{C}$ apresentando um excesso de ruído de $\left\langle\Delta^{2} \hat{q}_{+}\right\rangle$que impediu a violação do critério DGCZ.

\begin{tabular}{|c|c|c|}
\hline Temperatura & $\left\langle\Delta^{2} \hat{p}_{-}\right\rangle$ & $\left\langle\Delta^{2} \hat{q}_{+}\right\rangle$ \\
\hline $18^{\circ} \mathrm{C}$ & 0,44 & 22,9 \\
\hline $0{ }^{\circ} \mathrm{C}$ & 0,45 & 24,6 \\
\hline$-4{ }^{\circ} \mathrm{C}$ & 0,45 & 24,7 \\
\hline
\end{tabular}

Tabela 6.2: Resumo dos resultados das medidas, vistas nos gráficos 6.37, 6.38 e 6.39, indicando a não-violação do critério DGCZ. Com o resfriamento do cristal, verificou-se que o excesso de ruído de fase é mesmo proveniente do laser Diabolo.

redução da temperatura mostra que esse excesso de ruído não é originado do fenômeno de interação fônons-fótons, mas originado do próprio laser de bombeio.

No projeto inicial do teletransporte bicolor da Figura 5.4, a cavidade de filtro foi desconsiderada exatamente porque o bombeio não é ressonante na cavidade do OPO, ou seja, na dinâmica estocástica do DROPO [5, 14], ele é considerado um campo clássico e, como tal, seu excesso de ruído de fase não é acoplado aos gêmeos. Entretanto, a curva de transmissão 6.25 nos mostra que o DROPO2 não é perfeitamente "não-ressonante" para o verde, o que justifica o acoplamento do seu ruído de fase.

Para eliminar esse excesso de ruído, é necessário alinhar uma cavidade de 
filtro logo após a saída do laser ${ }^{25}$. Como veremos na próxima seção, a grande desvantagem de uma cavidade de filtro na montagem é a redução, em torno de $30 \%$, da potência do laser de bombeio.

A medida de $\left\langle\Delta^{2} \hat{p}_{-}\right\rangle$vista na tabela 6.28 indica o grande potencial do DROPO2 para servir como canal quântico do nosso protocolo de teletransporte. Entretanto, para continuar com ele e com a cavidade de filtro seria necessário que o laser tivesse mais potência de saída, visto o alto limiar requerido por esse OPO na condição de oscilação.

Infelizmente, tivemos que abrir mão de um OPO duplamente ressonante no teletrasnporte bicolor e mudamos para um triplamente ressonante. Conforme previamente discutido no capítulo 5, esse mudança requer a montagem de uma cavidade de análise para o verde que é refletido do TROPO para aproveitar a informação de fase contida nesse feixe.

\subsubsection{Cavidade de filtro para o bombeio}

Antes de falar sobre a construção dos OPOs triplamente ressonante, foi preciso reativar a cavidade de filtro para o bombeio, encontrada em montagens anteriores do grupo, conforme visto em [14, 70, 65, 72].

O nosso laser comercial Diabolo usado como bombeio possui uma largura de banda, fornecida pelo fabricante, de $1 \mathrm{KHz}$. Essa largura de linha é resultado do excesso de ruído do laser e encontra-se totalmente nas suas bandas laterais.

Para que o excesso de ruído do bombeio não acople nos feixes gêmeos e impeça a geração de emaranhamento,é necessário filtrar esse ruído.

A técnica [74] empregada para essa filtragem é usar uma cavidade ótica e travá-la em sua ressonância, de forma que suas bandas laterais sejam completamente refletidas pela cavidade.

Conforme vimos no capítulo 5, empregamos essa mesma técnica para separar as bandas laterais do feixe sinal do OPO, onde está contido o emaranhamento, da sua portadora, que é o feixe utilizado por Victor para produzir o estado quântico que será teletransportado por Alice.

A Figura 6.40 exibe um esquema dessa cavidade, assim como um gráfico do raio do feixe $\omega(z)$ em função da distância em relação à posição da cintura do laser. A cintura do feixe, como na cavidade de análise, situa-se também no centro.

\footnotetext{
${ }^{25}$ Atualmente, depois de mais de uma década de uso, nosso laser oferece somente uma potência de saída em torno de $650 \mathrm{~mW}$.
} 


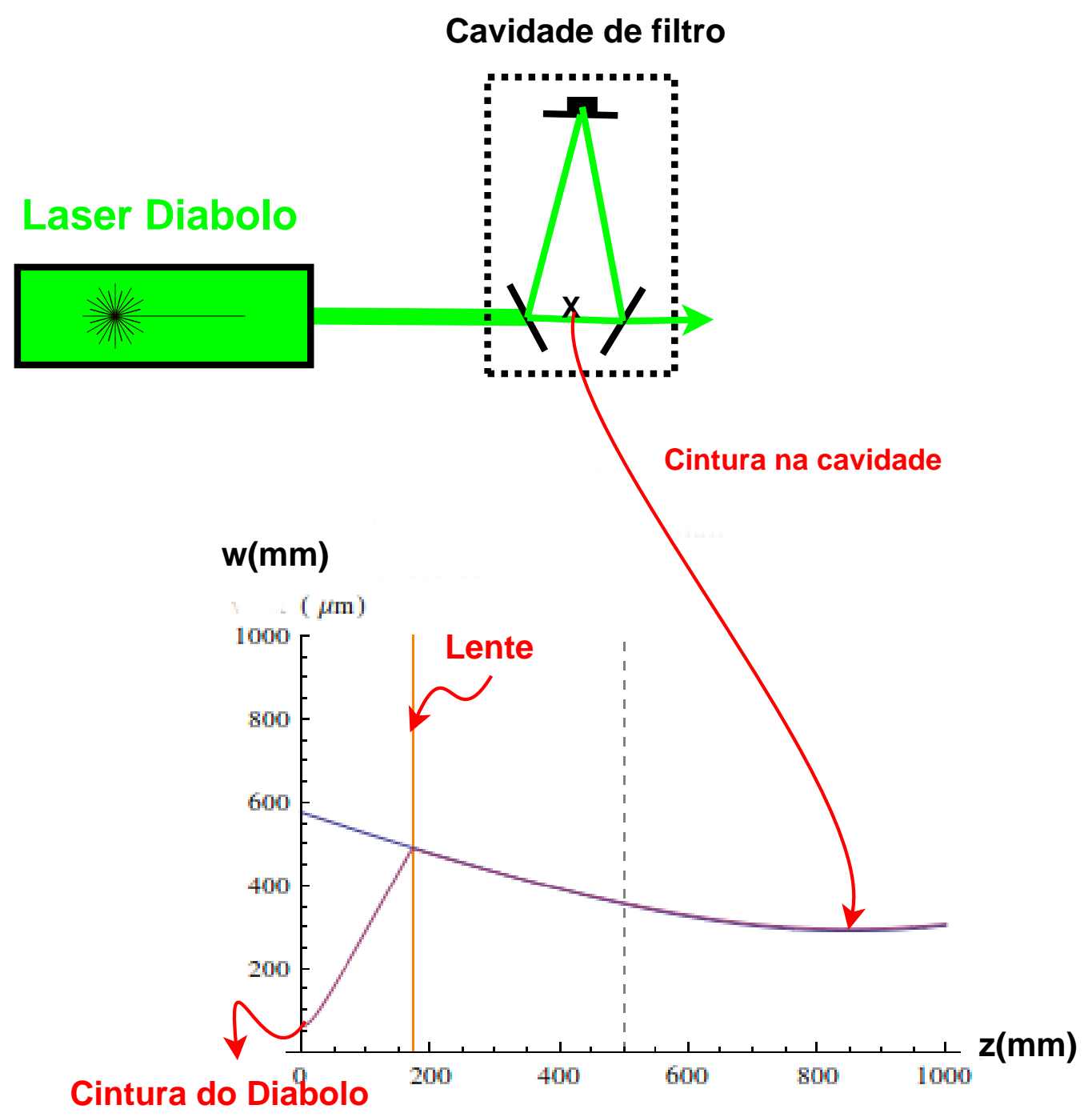

Figura 6.40: Esquema ilustrando a cavidade de filtro usada para eliminar o excesso de ruído do laser. Cavidade no formato de anel, cuja cintura do feixe encontra-se no centro. O gráfico exibe o raio, $\omega(z)$, do feixe de bombeio em função da distância da cintura do Diabolo até a cintura no interior da cavidade de filtro. O acordo de modo com o laser foi feito somente com uma lente de distância focal de $150 \mathrm{~mm}$. Cintura do Diabolo igual a $59,8 \mu \mathrm{m}$ e cintura do feixe na cavidade $289 \mu \mathrm{m}$. 


\subsubsection{Tentativa $3:$ TROPO1}

A nossa primeira tentativa de montagem de um OPO triplamente ressonante para servir de canal quântico do teletransporte foi com o TROPO1, cuja configuração de espelhos é ilustrada na Figura 6.41. A Figura 6.42 ilustra a transmissão com função da variação de fase para o TROPO1.

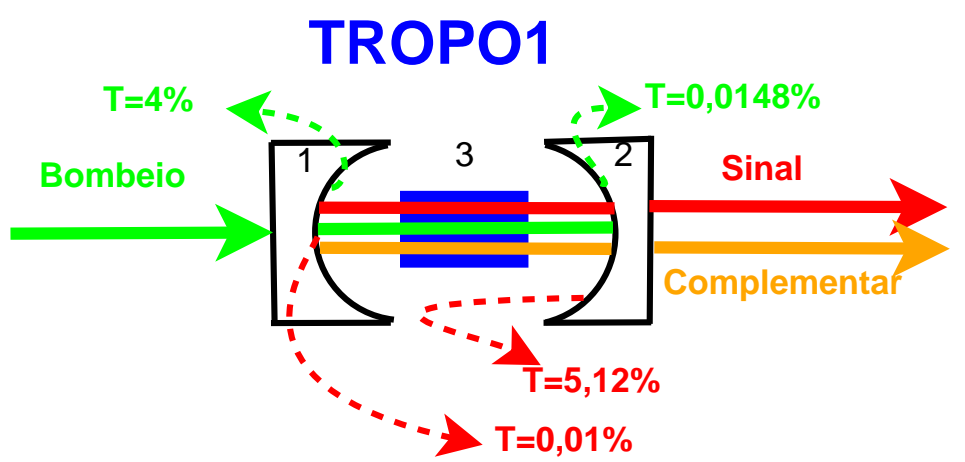

Figura 6.41: Configuração do TROPO1 montado no interior da câmara de vácuo. São vistas as transmitâncias para o verde e o IR dos espelhos côncavos 1 e 2 da cavidade montada para abrigar o cristal KTP, 3. Raio de curvatura de ambos espelhos é igual a 10mm. Sobre o espelho 2 é acoplado um PZT.

A ideia ao projetar o TROPO1 foi substituir apenas o espelho de entrada 1 do DROPO2 (Figura 6.24), mantendo o espelho de saída e o mesmo comprimento de Rayleigh (no caso, $z_{0}=3,57 \mathrm{~mm}$ para os gêmeos e para o $532 \mathrm{~nm}$.) do DROPO2. Com isso, os parâmetros do TROPO1 como finesse para o IR, $F S R$ e $B W$ são os mesmos que os do DROPO2, que atingiu o máximo de compressão de ruído já medido no LMCAL.

A oscilação ocorreu com um limiar otimizado em $41 \mathrm{~mW}$. Entretanto, devido a alta absorção do $532 \mathrm{~nm}$ pelo KTP não conseguimos travar a cavidade do TROPO1 no pico de transmissão do 532nm. Segundo a referência [73], o cristal KTP possui um coeficiente de absorção linear, aproximadamente, de $0,03 \mathrm{~cm}^{-1}$ para o $532 \mathrm{~nm}$. Assim, em uma volta completa na cavidade, sabendo-se que o nosso KTP tem $12 \mathrm{~mm}$ de comprimento, as perdas por absorção superam as perdas por transmissão do espelho 1. Dessa forma, optamos por projetar um novo TROPO, denominado de TROPO2, com um espelho 1 com maior transmitância para o verde.

A Figura 6.43 ilustra, por exemplo, uma situação de oscilação do TROPO1 com um limiar não-otimizado, em torno de $150 \mathrm{~mW}$, em que o efeito térmico, causado pela absorção do $532 \mathrm{~nm}$, provoca uma assimetria na produção dos gêmeos em função do sinal da varredura no PZT. 


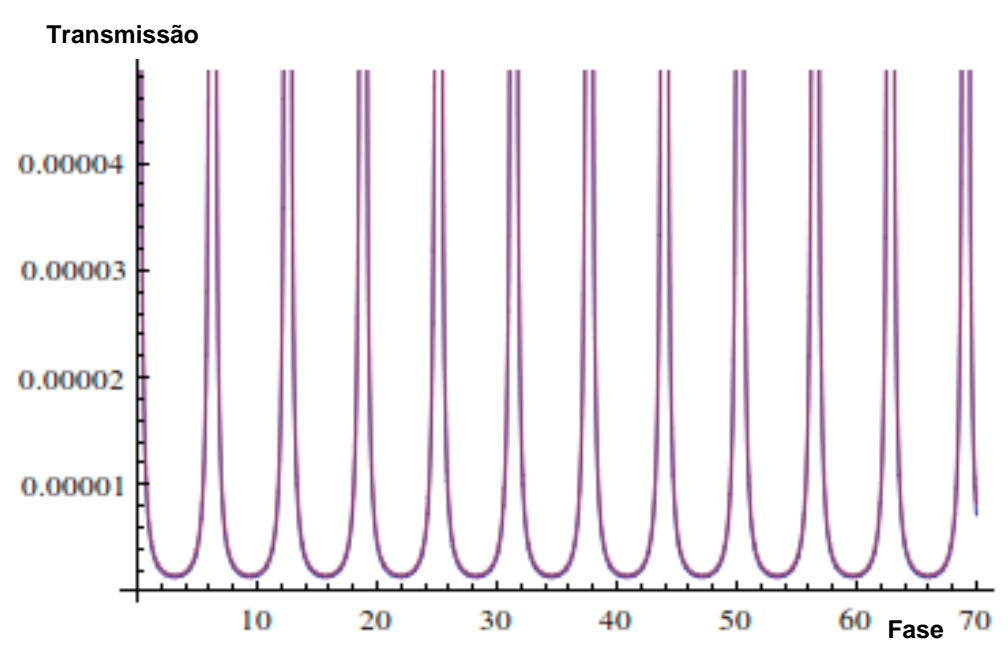

Figura 6.42: Picos de transmissão em função da fase para o $532 \mathrm{~nm}$, em rosa, e para o 1064nm, em azul, do TROPO1 ilustrando a condição de ressonância dos feixes no interior da cavidade. A fase dos feixes é variada através da mudança do comprimento da cavidade, que é realizada pelo PZT.

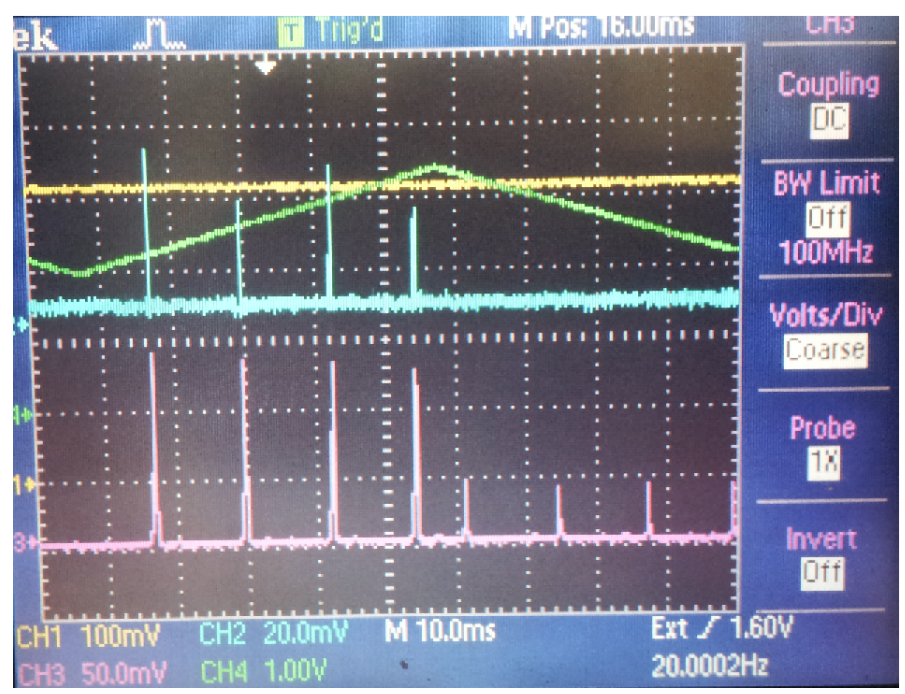

Figura 6.43: Figura meramente ilustrativa da imagem vista no osciloscópio dos picos de transmissão do $532 \mathrm{~nm}$, em rosa, e 1064nm, em azul, da cavidade do TROPO1. Em amarelo, sinal de transmissão da cavidade de filtro na condição de travamento. Em verde, sinal triangular fornecido ao PZT da cavidade do TROPO1. Com a rampa subindo (comprimento da cavidade aumentando), observa-se a oscilação do TROPO1, porém com a rampa descendo, os picos do 532nm são menores e não há oscilação do TROPO1. 


\subsubsection{Tentativa 4: TROPO2}

Com a montagem do TROPO1, percebemos o quão crítico é a questão da absorção do $532 \mathrm{~nm}$ pelo KTP e como isso prejudica o travamento da cavidade. Então, optamos pelo projeto de um outro OPO triplamente ressonante, TROPO2, com o espelho 1 com uma transmitância de $11 \%$ para o verde, de forma que as perdas por transmissão fossem maior que a absorção do cristal.

O esquema da montagem do TROPO2, com as transmitâncias, raios de curvatura dos espelhos e comprimento de Rayleigh $\left(z_{0}\right)$ empregado no interior do cristal, é visto na Figura 6.44. Esse OPO apresentou um limiar de oscilação em torno de $90 \mathrm{~mW}$.

A Figura 6.45 exibe a condição de ressonância na cavidade desse OPO em que são visualizadas ambas as funções de transmissão da cavidade para os dois comprimentos de onda.

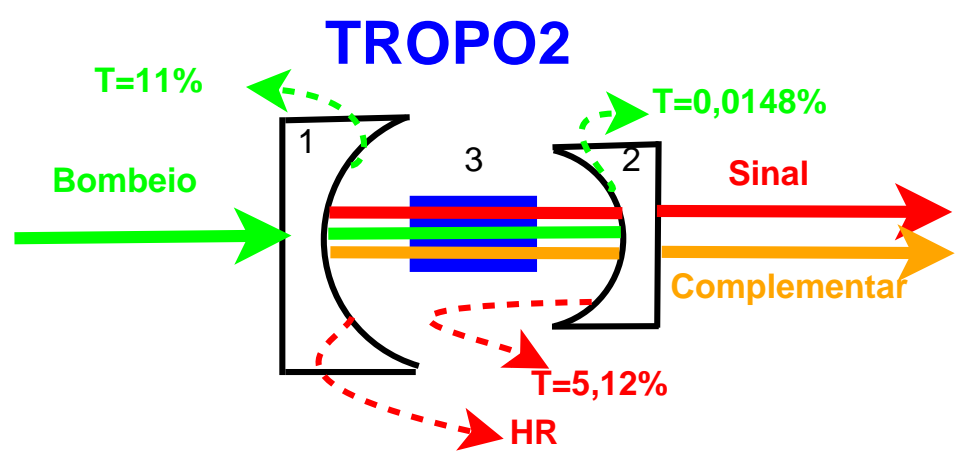

Figura 6.44: Configuração do TROPO2 montado no interior da câmara de vácuo. São vistas as transmitâncias para o verde e o IR dos espelhos côncavos 1 e 2 da cavidade montada para abrigar o cristal KTP, 3. Raio de curvatura do espelho 1 é igual a $25 \mathrm{~mm}$ e, do espelho 2,10mm . Sobre o espelho 2 é acoplado um PZT. Comprimento de Rayleigh $z_{0}=4,62 \mathrm{~mm}$.

Para testar se o TROPO2, de fato, pode servir como canal quântico do nosso protocolo de teletransporte, partimos para as medidas das quadraturas dos feixes: bombeio, sinal e complementar.

A fim de verificar a violação da desigualdade (5.3), montamos a configuração experimental vista na Figura 6.46 com a presença de mais uma cavidade de análise para o bombeio para a realização das medidas de $\left\langle\Delta^{2} \hat{p}_{-}\right\rangle$ e $\left\langle\Delta^{2}\left(\hat{q}_{+}-\alpha \hat{q}_{0}\right)\right\rangle$.

O procedimento para a realização da medida é o mesmo descrito para o DROPO2, porém com a diferença que há mais uma cavidade de análise, cujo o pico de transmissão deve ser também sincronizado com os picos das outras duas cavidades. 


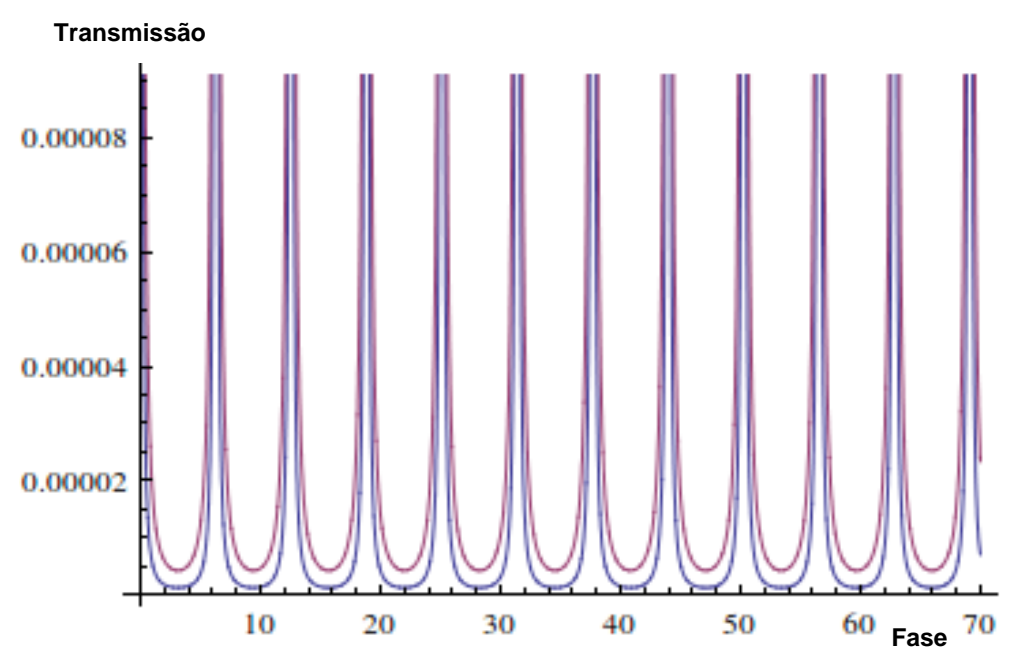

Figura 6.45: Picos de transmissão em função da fase para o 532nm, em rosa, e para o $1064 \mathrm{~nm}$, em azul, do TROPO2, ilustrando a condição de ressonância dos feixes no interior da cavidade. A fase dos feixes é variada através da mudança do comprimento da cavidade, que é realizada pelo PZT.

Com a detecção do bombeio, a verificação do emaranhamento do sistema é, como dito anteriormente, feita mediante a violação da desigualdade (5.3) com o uso da condição (5.7).

Os gráficos das Figuras 6.47 e 6.48 ilustram o melhor resultado obtido até agora nessa nova configuração, com o cristal a uma temperatura de $17^{\circ} \mathrm{C}$. A tabela 6.3 exibe um resumo com as medidas, referente a esses gráficos, mostrando que, mesmo com a presença da informação do bombeio, a desigualdade (5.3) não foi violada e o TROPO2 não pode ser, pelo menos a temperaturas dessa ordem, o canal quântico do protocolo. Porém, antes de descartar totalmente o TROPO2 como fonte é preciso fazer mais um teste e verificar se ainda é possível obter emaranhamento reduzindo a temperatura do cristal.

\begin{tabular}{|c|c|c|}
\hline$\left\langle\Delta^{2} \hat{p}_{-}\right\rangle+\left\langle\Delta^{2} \hat{q}_{+}\right\rangle$ & $\alpha$ & $\left\langle\Delta^{2} \hat{p}_{-}\right\rangle+\left\langle\Delta^{2}\left(\hat{q}_{+}-\alpha \hat{q}_{0}\right)\right\rangle$ \\
\hline $4,4(1)$ & $0,73(3)$ & $2,4(2)$ \\
\hline
\end{tabular}

Tabela 6.3: Melhor resultado, referente aos gráficos 6.47 e 6.48, obtido até agora com o TROPO2 com o cristal a uma temperatura de $17^{\circ} \mathrm{C}$. A informação do bombeio foi incluída, usando-se o valor de $\alpha$ fornecido pela equação (5.7). Apesar de se aproximar do limite de violação da desigualdade, mesmo assim não foi observado emaranhamento. 


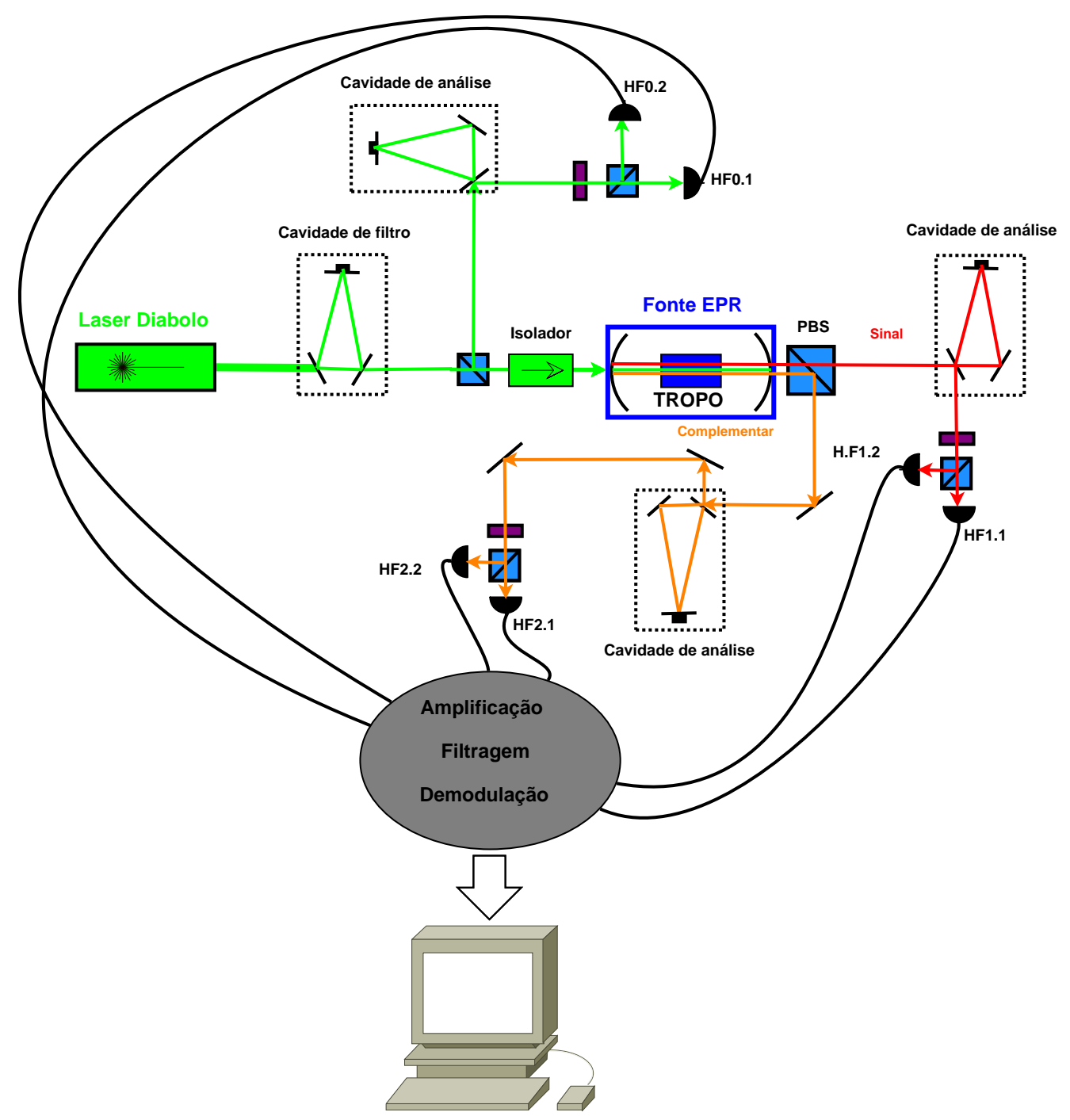

Figura 6.46: Padrão de cores adotados para os elementos óticos: retângulo verde (isolador ótico), cubo azul (PBS) e retângulo roxo (lâmina de meia onda). Esquema experimental para a realização das medidas $\left\langle\Delta^{2} \hat{p}_{-}\right\rangle$e $\left\langle\Delta^{2}\left(\hat{q}_{+}-\alpha \hat{q}_{0}\right)\right\rangle$ através dos sinais HF demodulados provenientes dos seis detectores vistos na montagem. Nessas medidas, o TROPO2 era travado e as três cavidades de análise eram varridas simultaneamente. Sinais DC omitidos na figura. 


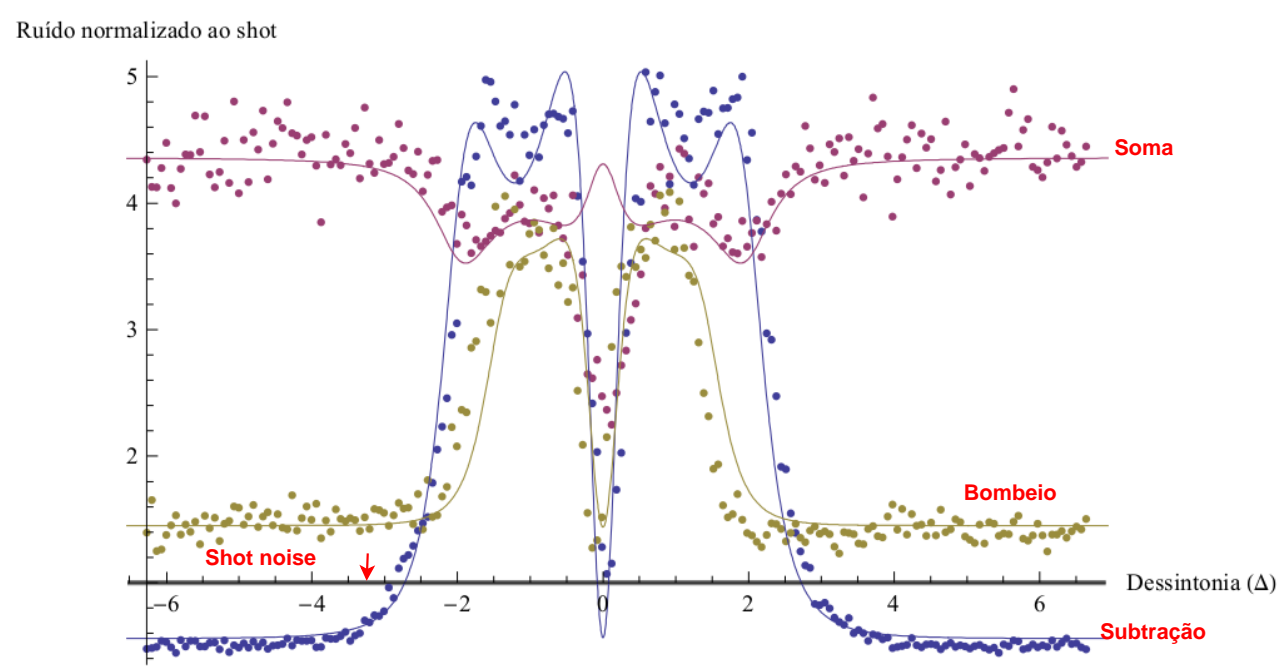

Figura 6.47: Gráfico com a melhor medida obtida, até o momento, com a varredura das três cavidades de análise com o cristal a $17^{\circ} \mathrm{C}$ de temperatura.

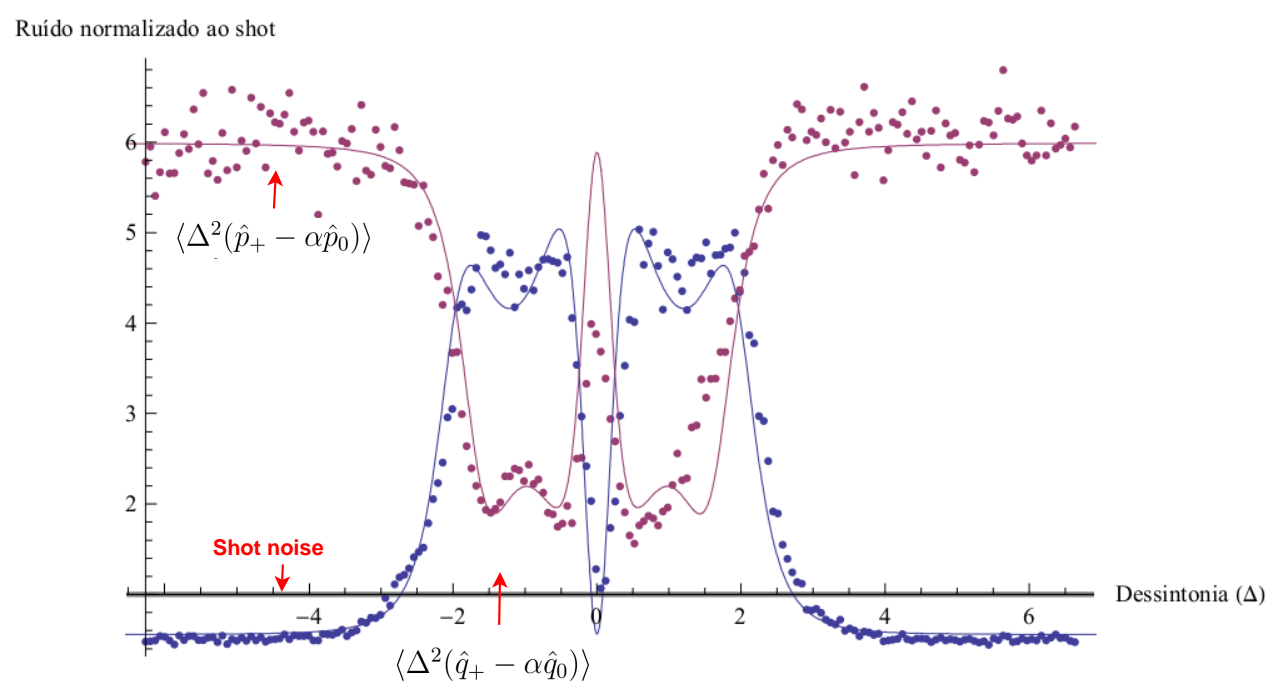

Figura 6.48: Gráfico referente as medidas exibidas no gráfico 6.47 levando em consideração a informação quântica contida no feixe de bombeio. 


\section{Capítulo 7}

\section{Conclusão}

O presente doutorado visou implementar experimentalmente um sistema de teletransporte no Laboratório de Manipulação de Átomos e Luz(LMCAL), usando um sistema denominado OPO, oscilador paramétrico ótico, como fonte de estados emaranhados, operando em um regime onde dois feixes de comprimentos de onda distintos são produzidos.

Esse tipo de operação permitirá implementar um tipo novo de teletransporte em variáveis contínuas, denominado teletransporte bicolor, que possibilitará a aniquilação do estado quântico, a ser teletransportado, em uma dada cor e sua recriação em uma cor diferente. A proposta inicial é uma demonstração de princípios, porém através dela vislumbrar-se-á a aplicação de outros esquemas de transmissão da informação quântica contida, por exemplo, em uma nuvem de átomos.

A maior parte do trabalho consistiu em desenvolver a instrumentação para a realização do teletransporte bicolor que compreendeu no projeto e construção da câmara de vácuo e de todas as cavidades óticas, na caracterização de detectores e moduladores eletro-óticos e no travamento mais robusto da cavidade de filtro, em que foi empregada a técnica de travamento Pound-Drever-Hall.

A proposta passou por uma alteração desde a ideia original concebida e essa mudança foi um dos principais resultados desse doutorado. Inicialmente, usaríamos um OPO duplamente ressonante como fonte de estados emaranhados do protocolo, porém o excesso de ruído de fase originado do laser comercial Diabolo e o alto limiar apresentado por esse OPO impossibilitou a montagem com esse tipo de OPO. Então, como uma alternativa para execução da proposta, resolvemos alterar a proposta inicial e montar um OPO triplamente ressonante.

O aparecimento de um terceiro campo, o verde refletido, forçou uma drástica reestruturação do projeto para aproveitar a informação quântica 
contida nesse feixe. Como consequência, o esquema experimental inicial foi acrescido de mais duas cavidades óticas e suas respectivas unidades de controle eletrônico.

As primeiras medidas de emaranhamento desse novo sistema foram realizadas e apresentaram um emaranhamento muito aquém do esperado para a realização do protocolo com sucesso. Atualmente, todos os nossos esforços estão concentrados em obter um nível de emaranhamento com esse TROPO suficiente para o prosseguimento da montagem. 


\section{Apêndice A}

\section{O papel da eletrônica}

Antes de começar a descrever de que forma são realizadas as medidas das quadraturas, amplitude e fase, do campo eletromagnético, é preciso analisar o papel essencial exercido pela eletrônica na questão do tratamento das correntes elétricas produzidas pela exposição dos detectores à luz incidente. Conforme discutido, toda a informação quântica é extraída da análise dessas correntes que são adquiridas e enviadas diretamente ao computador. Entretanto, do processo de detecção da luz à aquisição via computador, existe uma longa cadeia de processamento eletrônico do sinal elétrico que consiste principalmente de etapas de filtragens e amplificações.

Nesse apêndice, serão discutidas como essas etapas são realizadas e os tipos simples de circuitos que usamos no laboratório. Além disso, introduziremos um importante componente eletrônico, o amplificador operacional, que juntamente com o fotodiodo (abordado na seção 3.1), protagonizam a detecção em experimentos na área de Ótica Quântica em variáveis contínuas.

\section{A.1 Filtros eletrônicos: RC passa-alta, baixa e banda}

Durante o processamento de um dado sinal elétrico, gerado em um experimento qualquer, faz-se necessário frequentemente selecionar algumas bandas de frequências e remover certas componentes de frequência do sinal produzido. Para realizar essa função, são empregados circuitos eletrônicos denominados de filtros.

Existe vários tipos de filtros, de diferentes configurações e elementos, mas, para ilustrar, usaremos um circuito RC. Faremos isso por duas razões: primeiro, porque é o tipo de circuito que empregamos no tratamento das correntes geradas durante o processo de detecção e em outras etapas do 
processo, segundo, porque é possível ilustrar com esse único circuito, de forma simples, os três tipos de filtros que mais utilizamos: passa-alta, passa-baixa e o passa-banda.

A Figura A.1 ilustra um circuito RC alimentado por uma fonte AC externa, V. Esse circuito pode servir, como determinaremos a seguir, tanto como um filtro passa-alta quanto como um filtro passa-baixa. Como o próprio nome sugere, o filtro passa-alta seleciona altas frequências, em relação a uma dada frequência definida, conhecida como frequência de corte, e o filtro passabaixa seleciona somente as baixas frequências. Esse é um problema extremamente simples de física básica, porém de grande utilidade nos laboratórios. Verifiquemos como isso ocorre.

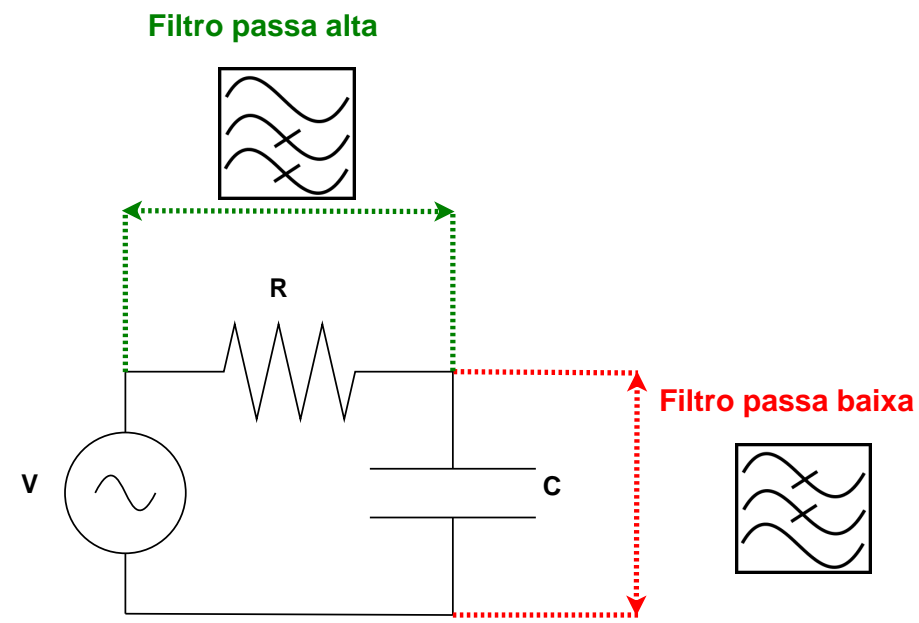

Figura A.1: Circuito RC alimentado por uma fonte periódica externa ilustrando os dois tipos possíveis de filtros do circuito nessa configuração: passaalta e passa-baixa. São também ilustrados nessa figura os símbolos que representam esses filtros.

Vamos usar a notação complexa, descrita na referência [15], em que todas as grandezas consideradas oscilam com a mesma frequência angular $\omega$ (igual a $2 \pi f, f$ frequência), e escrever $\mathbf{V}$ como:

$$
\mathbf{V}=\mathbf{V}_{m} e^{i \omega t}
$$

onde $\mathbf{V}_{m}$ é a amplitude da fonte, cuja fase é nula, por hipótese. A corrente elétrica do circuito é dada por:

$$
\mathbf{I}(t)=\mathbf{I}_{m} e^{i \omega t+\phi}
$$

Dessa forma, a equação diferencial de primeira ordem que governa o circuito 
RC é:

$$
\frac{d \mathbf{V}}{d t}=\frac{\mathbf{I}}{C}+R \frac{d \mathbf{I}}{d t}
$$

Para resolvê-la, é muito simples, substituimos as equações (A.1) e (A.2), e obtemos:

$$
\mathbf{V}=\mathbf{I}\left(R-\frac{i}{\omega C}\right)
$$

Dessa equação, identificamos imediatamente a impedância,Z, do circuito RC:

$$
\mathbf{Z}=\left(R-\frac{i}{\omega C}\right)
$$

Assim, a corrente elétrica é facilmente obtida da parte real de $\mathbf{I}(t)$ :

$$
R e[\mathbf{I}(t)]=\frac{\mathbf{V}_{m}}{\sqrt{R^{2}+\left(\frac{1}{\omega C}\right)^{2}}} \cos \left(\omega t-t g^{-1}\left(-\frac{1}{\omega R C}\right)\right)
$$

Verifiquemos agora a tensão medida nos terminais do resistor R da figura A.1. Chamaremos essa tensão de $\mathbf{V}_{\text {alta }}$ porque essa região do circuito comporta-se como um filtro passa-alta. A medida dessa tensão é, obviamente, a parte real de $\mathbf{V}_{\text {alta }}$ :

$$
\operatorname{Re}\left[\mathbf{V}_{\text {alta }}\right]=R R e[\mathbf{i}(t)]
$$

O limite da equação (A.7) quando a frequência tende a zero é nulo, o que indica que os sinais de baixas frequências são barrados por essa parte do circuito.

Antes de seguir com o raciocínio sobre o filtro passa-alta, precisamos definir uma das principais grandezas que se aprende no trabalho experimental, o ganho.

O ganho é uma grandeza de natureza complexa que diz respeito à resposta de um dado sistema a um dado sinal de entrada. Ele não é limitado somente a contextos onde todos os sinais envolvidos são elétricos, conforme será descrito no capítulo 6 com o EOM. Este dispositivo eletro-ótico, que servirá de canal clássico na implementação do teletransporte, é alimentado por um sinal de tensão e muda a fase da onda eletromagnética que o atravessa. O ganho, nesse caso, refere-se ao quanto de tensão é necessária para variar a fase da onda, dado por Radiano por Volt, $\frac{\mathrm{rad}}{\text { volt }}$.

Sendo assim, definimos o ganho, G, de forma genérica, que serve em qualquer contexto, como a razão entre o sinal de saída considerado e o sinal de entrada:

$$
\mathbf{G} \equiv \frac{\text { Saida }}{\text { Entrada }}=|\mathbf{G}| e^{i \Phi}
$$


Na maioria das situações experimentais, é muito comum o estudo somente de $|\mathbf{G}|$ como forma de caracterizar a função resposta do sistema em estudo, entretanto, em outras, é essencial também o estudo da fase $\Phi$ de $\mathbf{G}$.

No caso específico do filtro RC passa-alta, o ganho, denotado por $\mathbf{G}_{\text {alta }}$, é a razão entre $\mathbf{V}_{\text {alta }}$ e o sinal da fonte, $\mathbf{V}$, que é o sinal de entrada do circuito. Ele pode ser calculado com o uso das equações (A.6) e (A.7) e seu módulo é igual a:

$$
\left|\mathbf{G}_{\text {alta }}\right|=\frac{1}{\sqrt{1+\left(\frac{1}{R C \omega}\right)^{2}}}
$$

Nesse ponto definimos também a frequência de corte, $\mathbf{f}_{\text {corte }}$, que carateriza o filtro, como a frequência tal que o módulo do ganho seja igual a:

$$
|\mathrm{G}|=\frac{1}{\sqrt{2}}
$$

No filtro RC passa-alta, das equações (A.9) e (A.10), concluimos que a frequência de corte é:

$$
\mathbf{f}_{\text {corte }}=\frac{1}{2 \pi R C}
$$

A curva do módulo de $\left|\mathbf{G}_{\text {alta }}\right|$ em função da frequência é visualizada na figura A.2. Vemos que a frequência de corte é determinada pelos valores nominais dos componentes $\mathrm{R}$ e C. Na situação ilustrada nessa figura, R e C são tais que fixam uma $\mathbf{f}_{\text {corte }}$ igual a $12 \mathrm{MHz}$. Um circuito desse tipo simples $\mathrm{RC}$, exatamente com essa frequência de corte, é o responsável pela primeira filtragem do sinal gerado na detecção da luz pelo detector.

Analisemos agora a tensão medida nos terminais do capacitor da figura A.1. Chamaremos essa tensão de $\mathbf{V}_{\text {baixa }}$ pois essa parte do circuito comportase como um filtro passa-baixa:

$$
\mathbf{V}_{\text {baixa }}=\mathbf{V}\left(1-\frac{R \mathbf{Z}^{*}}{|\mathbf{Z}|^{2}}\right)
$$

Usando-se a equação (A.5), é trivial verificar que o limite de $\operatorname{Re}\left[\mathbf{V}_{\text {baixa }}\right]$ tende a zero quando a frequência tende ao infinito, confirmado seu papel de filtro passa-baixa.

Repetindo-se o mesmo procedimento do filtro passa-alta, determinamos o módulo do ganho do filtro passa-baixa:

$$
\left|\mathbf{G}_{b a i x a}\right|=\frac{1}{\sqrt{1+(\omega R C)^{2}}}
$$




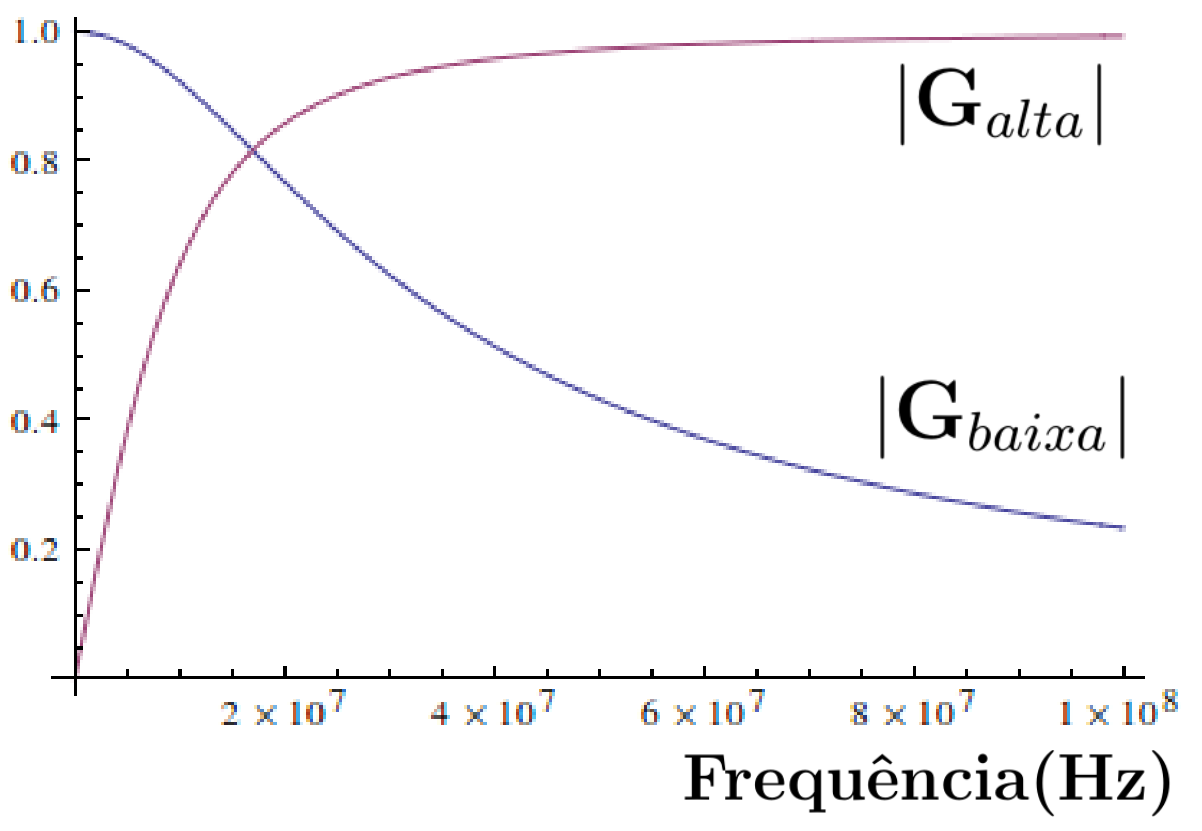

Figura A.2: Módulo dos ganhos dos filtros RC, da figura A.1, passa-alta e baixa em função da frequência. Valores dos componentes usados no circuito passa-alta: $R=485 \Omega$ e $C=27 p F$, e os valores no circuito passa-baixa: $R=5 K \Omega$ e $C=1,33 p F$. As frequências de corte são $12 \mathrm{MHz}$ e $24 \mathrm{MHz}$ respectivamente. 
Além disso, verificamos que a frequência de corte desse filtro é exatamente a mesma do filtro passa-alta, dada pela equação (A.11). A figura A.2 também ilustra a curva do módulo do ganho de um filtro RC passa-baixa com valores de $\mathrm{R}$ e $\mathrm{C}$ tais que definem uma frequência de corte de $24 \mathrm{MHz}$.

O último filtro a ser discutido aqui é o passa banda, utilizando os filtros $\mathrm{RC}$ anteriores. Às vezes, no tratamento do sinal elétrico de um certo experimento, é preciso remover tanto altas quanto baixas frequências e permitir somente a presença de uma banda de frequências. Esse é o nosso caso, por exemplo, com o Laser comercial, conhecido como Diabolo, que usamos para gerar os estados emaranhados que servirão de canal quântico do protocolo.

O feixe produzido pelo Diabolo é modulado em fase e intensidade na frequência $12 \mathrm{MHz}$ e no seu segundo harmônico $24 \mathrm{MHz}$. Se por um lado essas modulações são extremamente úteis no travamento em frequência de cavidades óticas, por outro, o excesso de ruído é sempre complicado em experimentos como os nossos que lidam com ruído quântico produzido pela luz. Para isso, usamos um filtro passa-banda $12-24 \mathrm{MHz}$ justamente para eliminar esse problema.

A figura A.3 mostra o esquema de um filtro passa-banda a partir da associação de um filtro RC passa-alta com um RC passa-baixa. Associandose os dois filtros, cujos ganhos foram ilustrados na Figura A.2, obtemos um RC passa-banda na região de frequências $12-24 \mathrm{MHz}$.

A saída do RC passa-banda, como visualizada na Figura A.3, é no capacitor $C_{2}$. Podemos concluir, dado o que foi descrito anteriormente, que a tensão nos terminais desse capacitor, chamado de $\mathbf{V}_{\text {banda }}$, pode ser escrito ${ }^{1}$ como:

$$
\mathbf{V}_{\text {banda }}=\mathbf{G}_{\text {alta }} \mathbf{G}_{\text {baixa }} \mathbf{V}
$$

Assim, o ganho desse filtro RC passa-banda é dado por:

$$
\mathbf{G}_{b a n d a}=\mathbf{G}_{\text {alta }} \mathbf{G}_{\text {baixa }}
$$

Da mesma forma como nos filtros anteriores, ilustramos o módulo do ganho desse filtro, $\left|G_{\text {banda }}\right|$, na Figura A.4.

\section{A.2 Amplificador operacional e ganho dos cir- cuitos}

Os componentes eletrônicos conhecidos como amplificadores operacionais(AO) são amplamente utilizados em todas as etapas da nossa montagem, desde a

\footnotetext{
${ }^{1}$ Ignorando-se o efeito de acoplamento das impedâncias.
} 


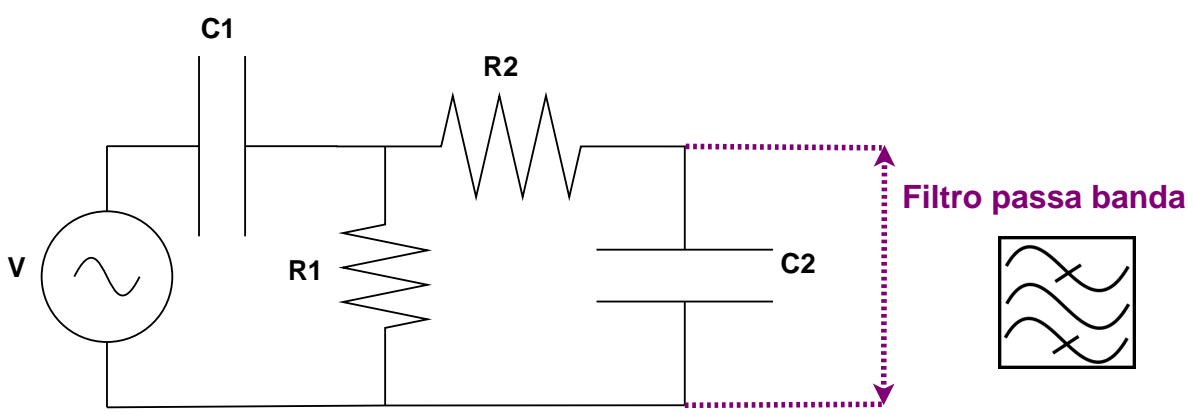

Figura A.3: Exemplo de um circuito passa-banda simples construído através da associação de um filtro RC passa-alta, de elementos $R_{1}$ e $C_{1}$, com um RC passa-baixa de elementos $R_{2}$ e $C_{2}$. A saída do filtro é no capacitor $C_{2}$. Do lado direito da figura, o símbolo que representa o filtro passa-banda.

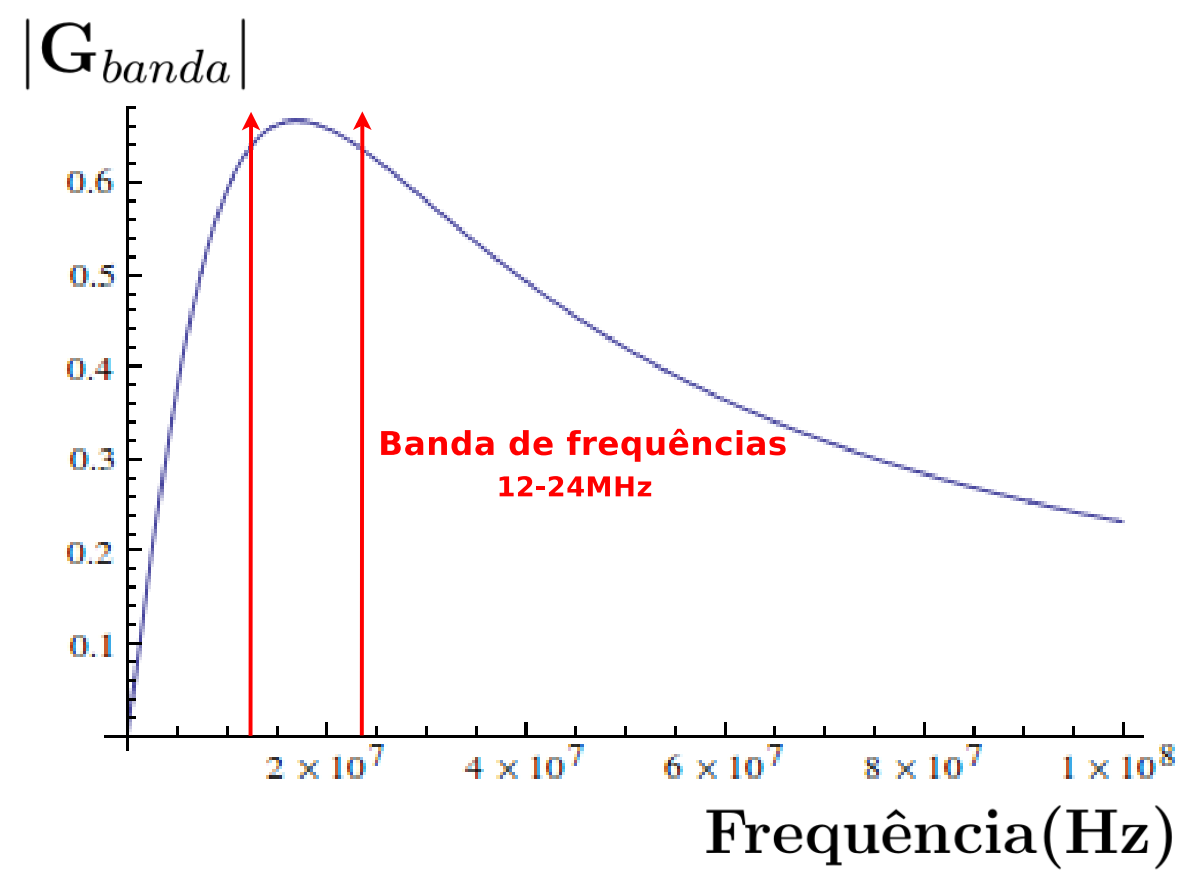

Figura A.4: Módulo do ganho do filtro passa-banda em função da frequência referente ao circuito da figura A.1. Esse filtro é construído a partir da associação de um filtro RC passa-alta com um RC passa-baixa. 
amplificação do sinal gerado no processo de detecção da luz, a estabilização de temperatura do cristal (usado na produção dos feixes gêmeos emaranhados) até o travamento em frequência de cavidades óticas.

Por ser tão crucial na realização do experimento, discutiremos de forma resumida esse componente, visando as aplicações na seção ?? e no capítulo 6. A Figura A.5 ilustra o símbolo desse componente que possui duas entradas, conhecidas como entrada inversora $(-)$ e não-inversora $(+)$, e somente uma saída. As tensões de entrada e saídas são denotadas por: $V_{-}, V_{+}, V_{S}$, respectivamente.

Os AOs são dispositivos complexos, na forma de circuitos integrados, que abrigam outros componentes tais como: transistores, resistores e capacitores. Na Figura A.5, é mostrado, em caráter meramente ilustrativo, o esquema elétrico desse dispositivo. O arranjo interno desses componentes nos AOs conferem a eles algumas propriedades importantes como:

- Alto ganho: $G=\frac{V_{S}}{V_{+}-V_{-}} \rightarrow \infty$, o que implica que as tensões $V_{+}$e $V_{-}$ são iguais idealmente;

- Alta impedância em cada entrada, o que significa que não há passagem de corrente elétrica na entrada dos AOs;

- Impedância de saída nula.

A real importância desses componentes aparece quando os mesmos estão associados a outros componentes eletrônicos. Veremos três exemplos de circuitos, muito usados no laboratório, onde aparece o AO, e suas funções. Eles são conhecidos como: amplificador-inversor( ou proporcional), diferenciador e integrador. Algumas vezes, eles surgem juntos em um único circuito conhecido como PID amplamente usado em esquemas de controle tais como em travamento de cavidades óticas, assunto abordado no capítulo 6. A Figura A.6 mostra esses circuitos separados.

Da observação dos circuitos da Figura A.6 e utilizando as principais características do AO, listadas acima, é possível escrever as equações que caracterizam cada circuito:

(a) Proporcional(ou amplificador):

$$
V_{S}=-\left(\frac{R}{r}\right) V_{E}
$$

Como o próprio nome indica, esse circuito amplifica/reduz o sinal de tensão de entrada, $V_{E}$, por um fator que depende da razão entre os valores nominais das resistências as quais o AO está associado. Além disso, o sinal negativo indica a inversão do sinal de entrada. 


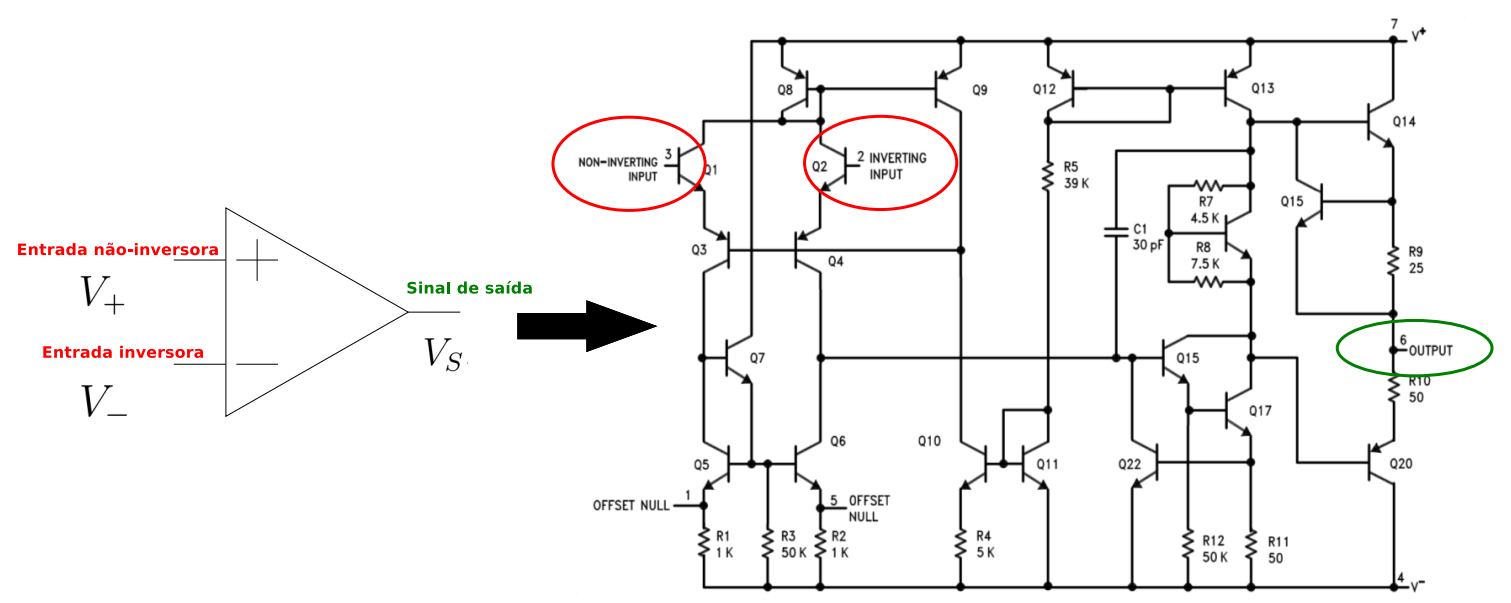

Figura A.5: Do lado esquerdo da figura, é visto a representação gráfica de um amplificador operacional. A entrada $(+)$ é a não-inversora e a $(-)$ a inversora. O dispositivo apresenta somente uma saída. Do lado direito, o esquema eletrônico interno de um amplificador operacional onde são vistos, em vermelho, as entradas e, em verde, a saída.

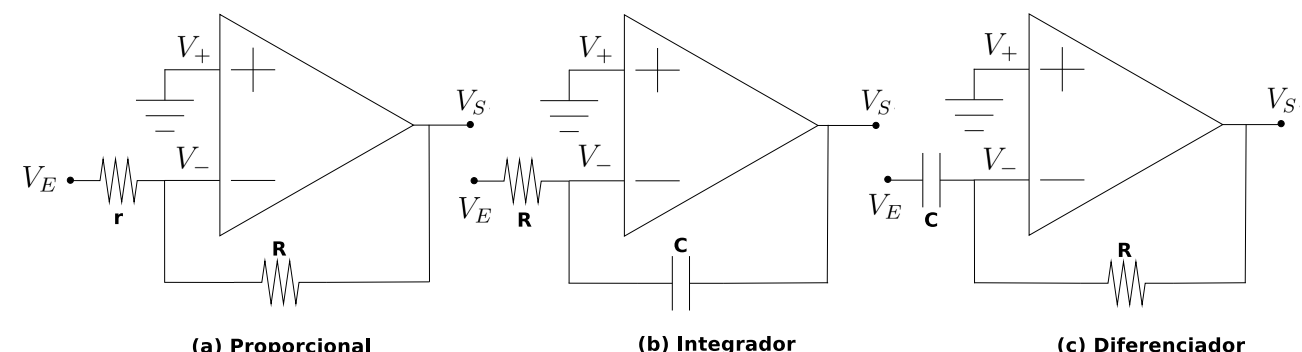

Figura A.6: Exemplos de circuitos muito usados nos laboratórios de Ótica Quântica com o emprego dos AOs. Em (a), temos o circuito amplificador, em (b), o circuito que funciona como integrador e, em (c), o diferenciador. Muitas vezes, encontra-se esses três circuitos associados, em um circuito conhecido como PID, que serve como circuito de controle no travamento de cavidades óticas. As tensões $V_{-}, V_{+}, V_{E}$ e $V_{S}$ representam as tensões da inversora, não-inversora, a tensão de entrada no circuito e a de saída respectivamente. Nos três circuitos a entrada não inversora está aterrada, logo: $V_{+}=V_{-}=0$. 
(b) Integrador:

$$
V_{S}=-\left(\frac{1}{R C}\right) \int V_{E} d t
$$

Esse circuito integra o sinal de entrada e depende do inverso da constante $R C$.

(c) Diferenciador:

$$
V_{S}=-R C\left(\frac{d V_{E}}{d t}\right)
$$

Finalmente, o circuito usado para diferenciar sinais de tensão que também depende da constante $R C$.

Os AOs também podem aparecer em circuitos com outras funções que não foram descritas aqui como em circuitos somadores e subtratores, por exemplo.

\section{A.3 Função de transferência e gráfico de Bode}

Como observado nas equações diferenciais que descrevem a dinâmica dos circuitos, existe uma dificuldade para determinar o ganho desses sistemas usando a definição (A.8). Uma forma de contonar-se esse problema é calcular a chamada função de transferência do circuito. Em engenharia elétrica, essas funções são muito utilizadas exatamente porque simplificam o cálculo do ganho dos circuitos com equações diferenciais normalmente mais complicadas de se resolver.

O principal fundamento por detrás do uso dessas funções de transferência reside nas propriedades de linearidade e invariância no tempo dos sistemas elétricos com os quais trabalhamos no laboratório. Para explicar essas propriedades nesse contexto eletrônico, suponhamos que $u(t)$ seja um dado sinal elétrico, produzido em um experimento, que interage com um dado sistema, um circuito eletrônico, por exemplo. Da interação sinal-sistema surge uma função resposta, que denotaremos ${ }^{2}$ por $y(t)$. Isso é o que ocorre quando filtramos, amplificamos, integramos ou diferenciamos o sinal elétrico. A figura A.7 esquematiza os sinais de entrada e saída e alguns exemplos de sistemas elétricos vistos até agora.

Como sabemos, sistemas lineares obedecem ao princípio de superposição, ou seja, se dois (ou mais) sinais de entrada $u_{1}(t)$ e $u_{2}(t)$ produzem, ao interagir com um dado sistema, sinais de saída $y_{1}(t)$ e $y_{2}(t)$, então $u_{1}(t)+u_{2}(t)$ resulta em $y_{1}(t)+y_{2}(t)$.

\footnotetext{
${ }^{2}$ Usaremos sempre as mesmas nomenclaturas adotadas nos livros de engenharia elétrica.
} 


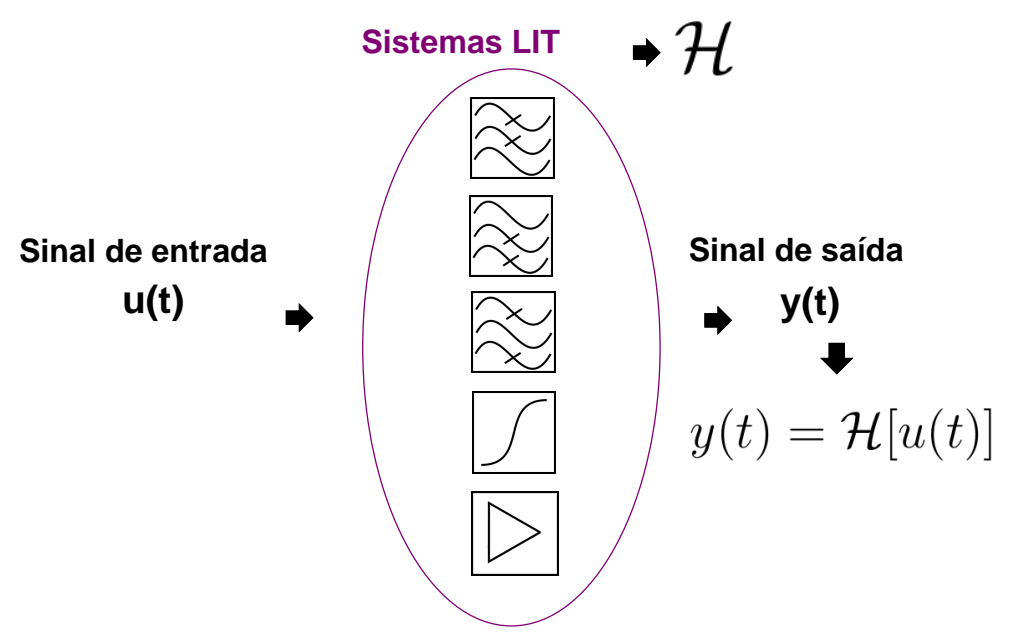

Figura A.7: Esquema que ilustra o processamento de um sinal elétrico. Um sinal elétrico produzido, $u(t)$, passa por um tratamento eletrônico em um dado sistema e produz um sinal de saída $y(t)$. Sistemas representados nas figuras: filtros passa baixa, passa alta, passa banda, integrador e amplificador.

Já por invariância no tempo, entende-se que a dinâmica do sistema é a mesma em qualquer instante de tempo [16]. Ou seja, se o sinal $u(t)$, que interage com o sistema e produz $y(t)$, sofrer um atraso de $\tau$, então a saída também sofrerá o mesmo atraso: $u(t-\tau) \rightarrow y(t-\tau)$.

Sistemas lineares e invariantes no tempo são conhecidos como $\operatorname{LIT}[16,17]$ e, ao interagir com sinais de entrada, não criam novas frequências, apenas modificam a amplitude e fase desses sinais. ${ }^{3}$

Se pensarmos no nosso experimento, veremos que, lidar com sistemas(circuitos) do tipo LIT, é fundamental para recuperar a informação quântica contida na corrente elétrica gerada durante o processo de detecção da luz. Isso porque a transmissão desse sinal até o computador deve ser tal que não o distorça e, assim, podemos garantir que a análise espectral da corrente gerada é, de fato, devido à natureza quântica da luz e não o produto do tratamento eletrônico sofrido pelo sinal.

Para definir a função de transferência, é preciso antes fazer algumas considerações a respeito da relação entre as funções de entrada, $u(t)$, e saída, $y(t)$, de um sistema linear $(L I T)$. Usaremos, a seguir, a dedução vista nas referências [16] e [17] para determinar de que forma esses sinais estão rela-

\footnotetext{
${ }^{3} \mathrm{E}$ importante destacar que saturações ou não-linearidades em dispositivos eletrônicos desses circuitos implicam na saída desse regime linear $(L I T)$ por definição.
} 
cionados entre si através da função de transferência. Veremos que a teoria que trata de sistemas lineares em si é a mesma que usamos muitas vezes em física, só que aplicada ao contexto elétrico.

Para isso, consideremos, inicialmente, que o sinal de entrada $u(t)$ é escrito em termos do delta de Dirac:

$$
u(t)=\int_{-\infty}^{+\infty} u(\tau) \delta(t-\tau) d \tau
$$

Vamos supor que qualquer circuito do tipo LIT mapeia os sinais de entrada, $u(t)$, em sinais de saída, $y(t)$, por meio de um operador linear, denotado por $\mathcal{H}$. Dessa forma, qualquer sinal $y(t)$ que deixa o sistema é sempre escrito como:

$$
y(t)=\mathcal{H}[u(t)]
$$

Substituindo-se a equação (A.19) na equação (A.20), temos que:

$$
y(t)=\mathcal{H}\left[\int_{-\infty}^{+\infty} u(\tau) \delta(t-\tau) d \tau\right]
$$

Como o operador $\mathcal{H}$ é linear e só atua sobre sinais que são funções do tempo $t$, então a equação (A.21) é igual a:

$$
y(t)=\left[\int_{-\infty}^{+\infty} u(\tau) \mathcal{H}[\delta(t-\tau)] d \tau\right]
$$

Quando o sinal de entrada no sistema é um delta de Dirac, $u(t)=\delta(t)$, o sinal de saída é denotado por $h(t)$ e chamado de resposta impulsiva:

$$
h(t)=\mathcal{H}[\delta(t)]
$$

Como o sistema é invariante no tempo, a resposta impulsiva pode também ser escrita deslocada no tempo de $\tau$ :

$$
h(t-\tau)=\mathcal{H}[\delta(t-\tau)]
$$

Assim, a equação (A.22) pode, finalmente, ser expressa como:

$$
y(t)=\left[\int_{-\infty}^{+\infty} u(\tau) h(t-\tau) d \tau\right]
$$

Realizando-se uma mudança de variáveis simples, a equação acima também pode ser escrita, alternativamente, como:

$$
y(t)=\left[\int_{-\infty}^{+\infty} u(t-\tau) h(\tau) d \tau\right]
$$


Essa expressão indica que o sinal de saída, $y(t)$, de um sistema LIT é a convolução entre o sinal de entrada, $u(t)$, e a função $h(t)$, que é a resposta impulsiva do sistema. Se, por exemplo, entrarmos com um sinal do tipo $u(t)=e^{s t}[17]$, em que $s=\sigma+i \omega$, a equação (A.26) resulta em:

$$
y(t)=H(s) e^{s t},
$$

em que:

$$
H(s)=\left[\int_{-\infty}^{+\infty} h(\tau) e^{-s \tau} d \tau\right]
$$

A função $H(s)$ é a função de transferência do circuito. Obviamente, para calcular $H(s)$ não é necessário resolver a equação (A.28), basta assumir uma solução do tipo (A.27), substituir na equação diferencial do circuito e obter diretamente $H(s)$ [17]. Ao longo desse texto, usaremos sempre essa propriedade para determinar as funções de transferência dos circuitos eletrônicos utilizados na nossa montagem experimental. A tabela A.1 exibe as funções de transferência, $H(s)$, de todos os circuitos descritos até agora.

Resumidamente, a função de transferência é a razão entre o sinal de entrada e o sinal de saída. Expressando-se em termos de $s$, o sinal de entrada é denotado por $U(s)$ e o de saída por $Y(s)$, e $H(s)$ é dado por:

$$
H(s)=\frac{Y(s)}{U(s)}
$$

Em essência, comparando-se essa equação com a definição do ganho, fonecida pela equação (A.8), percebemos que, na verdade, $H(s)$ é o próprio ganho do sistema LIT em questão com a vantagem de ser muito mais fácil de se determinar.

Toda essa abordagem teórica feita acima justifica uma técnica que normalmente utilizamos para determinar, experimentalmente, o módulo do ganho de um dado circuito $L I T$ e sua respectiva fase. A técnica refere-se em injetar um sinal de tensão do tipo senoidal no circuito com uma amplitude sempre fixa e variar sua frequência. A amplitude e a fase do sinal de saída são medidos como função da variação de frequência. Essa técnica é importante principalmente por ser muito empregada para caracterizar circuitos de controle, aqueles responsávis pelo travamento de cavidades óticas e pelo controle de temperatura.

Uma forma de se representar a função de transferência de um sistema LIT é graficar o seu módulo e sua fase em função da frequência em escala $l o g-l o g$, conhecido como gráfico de Bode. Em geral, esse gráfico é apresentado em uma unidade de medida conhecida como decibel, que compara os sinais de saída do circuito com o sinal de entrada(referência) usado para caracterizar $H(s)$. 


\begin{tabular}{|l|l|}
\hline Tipo de circuito & $H(s)$ \\
\hline Proporcional & $-\frac{R}{r}$ \\
\hline Integrador & $-\frac{1}{R C s}$ \\
\hline Diferenciador & $-R C s$ \\
\hline RC passa-alta & $\frac{R C s}{(1+R C s)}$ \\
\hline RC passa-baixa & $\frac{1}{(1+R C s)}$ \\
\hline
\end{tabular}

Tabela A.1: Funções de transferência dos circuitos estudados.

No caso em que o sinal é do tipo tensão ou corrente elétrica, a medida em $d B$ é definida como:

$$
\operatorname{Medida}(d B)=20 \log \left(\frac{\text { Sinal }}{\text { Referência }}\right)
$$

As Figuras A.8 e A.9 exibem os gráficos de Bode dos circuitos da tabela A.1.

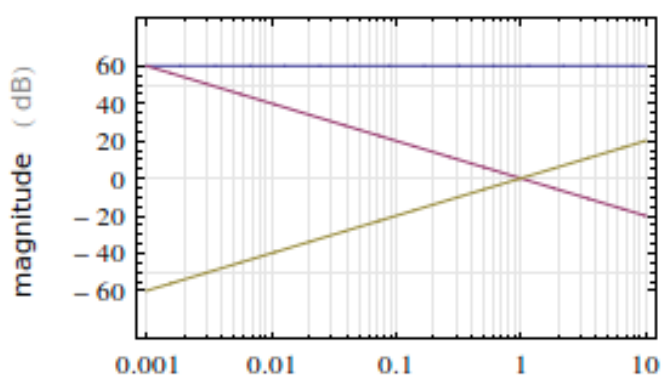

(a)

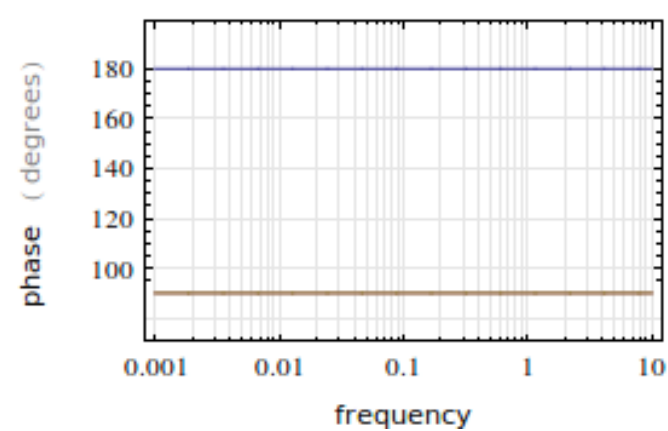

(b)

Figura A.8: Em (a), os gráficos de Bode das funções de transferência dos três circuitos ilustrados na Figura A.6 para os seguintes valores: $R=1 M \Omega, r=$ $1 K \Omega, C=1 \mu F$. Curva azul refere-se ao proporcional, a rosa ao integrador e a amarela ao diferenciador. Em (b), são vistos os respectivos gráficos da fase, onde mesmo padrão de cores é obedecido. 


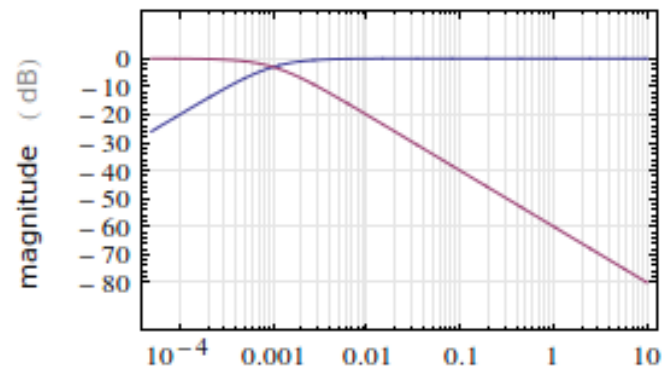

(a)

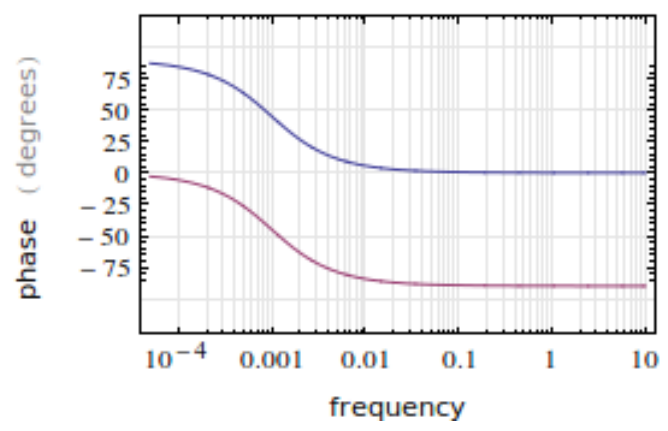

(b)

Figura A.9: Em (a), os gráficos de Bode das funções de transferência dos filtros RC passa alta e baixa, previamente estudados, ilustrados na Figura A.1 para os mesmos valores $R$ e $C$ da Figura A.8: $R=1 M \Omega$ e $C=1 \mu F$. Curva azul refere-se ao passa alta, a rosa ao passa baixa. Em (b), são vistos os respectivos gráficos da fase, onde mesmo padrão de cores é obedecido. 


\section{Apêndice B}

\section{Amplificação e segunda filtragem do sinal}

Vejamos mais detalhadamente de que forma ocorre a etapa de amplificação e a segunda filtragem do sinal vista no circuito simplificado da Figura 3.6. Trataremos somente do caso $i_{H F}$ por ser o sinal onde se encontra a informação quântica do feixe detectado na forma de ruído. Para não tornar a abordagem repetitiva omitimos o tratamento do caso $i_{D C}$, entretanto, o procedimento com esse sinal é completamente análogo.

O sinal $i_{H F}$ é muito pouco intenso para poder ser medido pelo nosso método de medida via computador, assim, é essencial que esse sinal passe por uma etapa de amplificação. Só para ter uma ideia da sua ordem de grandeza, segundo a referência [18], um sinal HF típico é da ordem de $10^{-7}$ do sinal DC.

A etapa de amplificação e filtragem ocorre por meio de um circuito eletrônico [22] um pouco mais complexo mostrado na Figura B.1. Nela podemos ver que a corrente $i_{H F}$ se divide em duas, $i_{1}$ e $i_{2}$, e que o sinal de saída é uma tensão elétrica, $V_{S}$. Esse processo em que a corrente $i_{H F}$ transforma-se em um sinal de tensão é conhecido como amplificação de transimpedância e acontece por conta das características do amplificador operacional anteriormente abordadas.

Observando-se o circuito da Figura B.1, vemos que:

$$
i_{H F}=C_{1} \frac{d V_{1}}{d t}
$$

lembrando que:

$$
i_{H F}=i_{1}+i_{2}
$$

Verificamos que:

$$
i_{1}=-\frac{V_{S}}{R_{3}} e,
$$




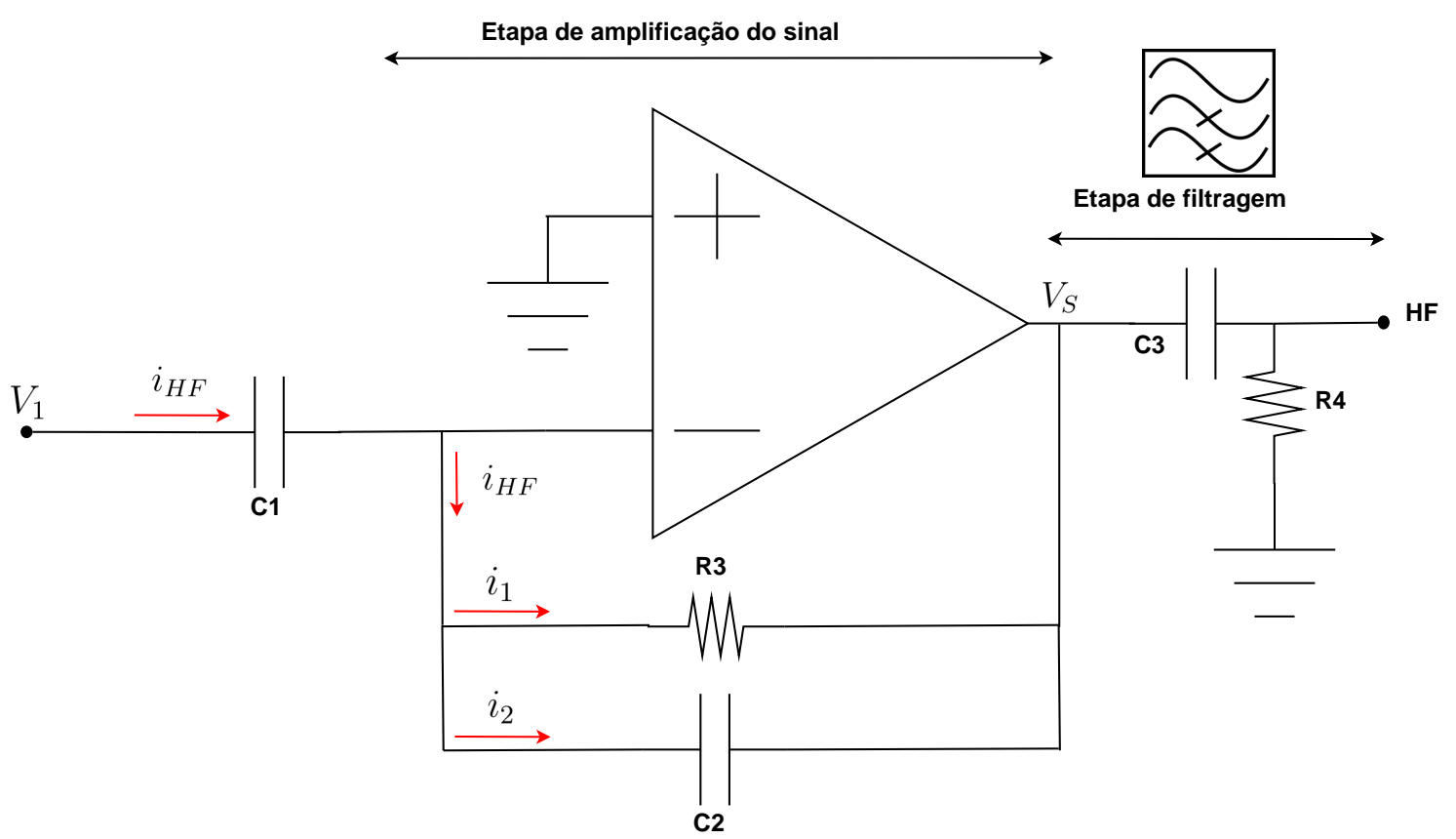

Figura B.1: Circuito de amplificação e filtragem do sinal $H F$. O sinal de entrada $i_{H F}$ é inicialmente amplificado e transformado em tensão de saída $V_{S}$. Após a amplificação, o sinal ainda passa por um segundo processo de filtragem eletrônica feita novamente por um circuito do tipo RC passa alta. 


$$
i_{2}=-C_{2} \frac{d V_{S}}{d t}
$$

Assim, substituindo-se as equações (B.3) e (B.3) na equação (B.2), temos, finalmente a equação diferencial que governa a tensão de saída, $V_{S}$, como função da tensão de entrada, $V_{1}$ :

$$
C_{1} \frac{d V_{1}}{d t}=-\frac{V_{S}}{R_{3}}-C_{2} \frac{d V_{S}}{d t}
$$

Como esse circuito é do tipo LIT, então usaremos a técnica descrita na seção A para simplificar a solução de (B.5). Para isso, façamos:

$$
V_{1}=e^{s t}
$$

e

$$
V_{S}=H(s) e^{s t}
$$

Das equações B.5,B.6 e B.7, calculamos a função de transferência $H(s)$ do sistema:

$$
H(s)=\frac{R_{3} C_{1} s}{\left(1+R_{3} C_{2} s\right)}
$$

De posse da função $H(s)$, podemos fazer o gráfico de Bode para os valores de $R_{3}, C_{1}$ e $C_{2}$ da nossa montagem atual. A Figura B.2 exibe esse gráfico. Para

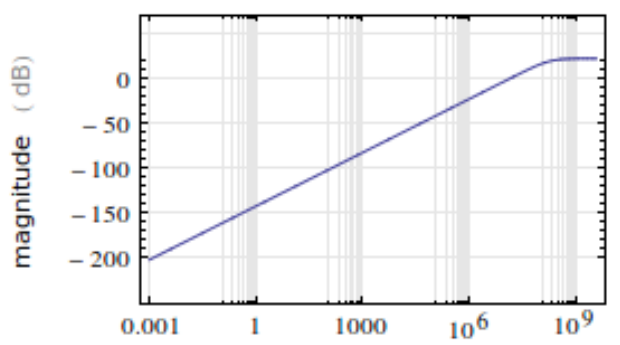

(a)

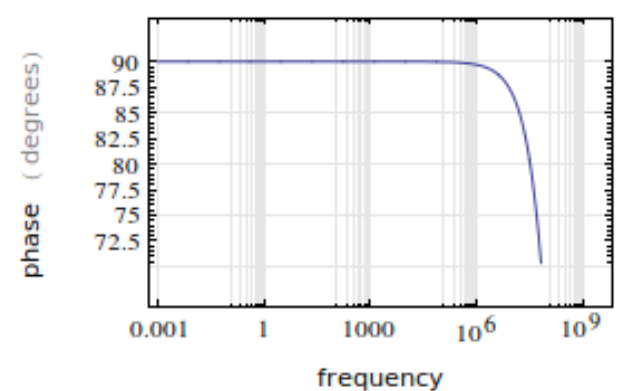

(b)

Figura B.2: Em (a), o gráfico de Bode da função de transferência do circuito, ilustrado na figura B.1, responsável pela amplificação do sinal HF. Valores de $R_{3}, C_{1}$ e $C_{2}: 2,7 k \Omega, 27 p F$ e $2 p F$ respectivamente. Em (b), é visto o gráfico da fase.

finalizar essa etapa, o sinal amplificado passa ainda por mais uma segunda filtragem realizada por mais um filtro RC passa alta, conforme visualizado na Figura B.1. Os valores nominais de $R_{4}, C_{3}$ são $100 \Omega$ e $150 p F$ respectivamente, o que fornece uma frequência de corte de $11 \mathrm{MHz}$. 


\section{Referências Bibliográficas}

[1] A. S. Villar, L. S. Cruz, K. N. Cassemiro, M. Martinelli, and P. Nussenzveig, Generation of Bright Two-Color Continuous Variable Entanglement, Phys. Rev. Lett. 95, 243603 (2005).

[2] A. S. Villar, M. Martinelli, C. Fabre, and P. Nussenzveig, Direct Production of Tripartite Pump-Signal-Idler Entanglement in the AboveThreshold Optical Parametric Oscillator, Phys. Rev. Lett. 97, 140504 (2006).

[3] A. S. Villar, K. N. Cassemiro, K. Dechoum, A. Z. Khoury, M. Martinelli, P. Nussenzveig, Entanglement in the above-threshold optical parametric oscillator, J. Opt. Soc. Am. B 24, 249 (2007).

[4] K. N. Cassemiro, A. S. Villar, P. Valente, M. Martinelli, and P. Nussenzveig, Experimental observation of three-color optical quantum correlations, Opt. Lett. 32, 695 (2007).

[5] Marcelo Martinelli, Compressão de ruído quântico e efeitos transversos em osciladores paramétricos óticos, Tese de doutorado (Universidade de São Paulo) (2002).

[6] A. S. Coelho, F. A. S. Barbosa, K. N. Cassemiro, A. S. Villar, M. Martinelli, P. Nussenzveig, Three-color entanglement, Science, 326 (2009).

[7] A. Furusawa, J. L. Sørensen, S. L. Braunstein, C. A. Fuchs, H. J. Kimble, E. S. Polzik, Unconditional quantum teleportation, Science 282,706 (1998).

[8] A. S. Villar, The conversion of phase to amplitude fluctuations of a light beam by an optical cavity, Am. J. Phys.76 (10) (2008).

[9] A. F. R. de Toledo Piza, Mecânica Quântica, 2. ed, São Paulo, Editora da Universidade de São Paulo, 2009. 
[10] C. C. Gerry, P. L. Knight, Introductory quantum optics, Cambridge University Press, Cambridge, United Kingdom (2005).

[11] Rodney Loudon, Quantum Theory of light, Oxford University Press (2000).

[12] M. O. Scully and M. S. Zubairy, Quantum Optics, Cambridge University Press, Cambridge, United Kingdom (1997).

[13] Mark Fox, Quantum Optics an Introduction, Oxford Master Series in Atomic, Optical and Laser Physics (2006).

[14] Alessandro S. Villar, Emaranhamento multicor entre feixes intensos de luz, Tese de doutorado (Universidade de São Paulo) (2008).

[15] H. M. Nussenzveig, Curso de Física Básica 3-Eletromagnetismo, 2. ed, São Paulo, Editora Edgard Blücher Ltda, 2001.

[16] L. Q. Orsini, A. F. Kohn, J. C. T. B. Moraes, Sistemas e sinais I, Apostila usada na disciplina PTC 2307 da Escola Politécnica da USP, 2013.

[17] G. F. Franklin, J. D. Powell, A. Emami-Naeini, Sistemas de controle para engenharia, tradução: Fernando de Oliveira Souza, revisão técnica: Antonio Pertence Júnior, 6. ed, São Paulo, Bookman Editora Ltda, 2013.

[18] H.-A. Bachor, A Guide to Experiments in Quantum Optics, Weinheim - Federal Republic of Germany: Wiley-VCH, 1998.

[19] High Speed InGaAs Photodiodes, Datasheet da Epitaxx, Optoeletronic Devices.

[20] Jerald Graeme, Photodiode Amplifiers- OP AMP Solutions, McGrawHill, 1996.

[21] Gilbert Grynberg, Alain Aspect, and Claude Fabre, Introduction to Quantum Optics from the Semi-classical Approach to Quantized Light, Cambridge University Press, Cambridge, United Kingdom (2010).

[22] M. Martinelli, User's Guide to Quadrant Photodetectors and Demodulation Circuit, Apostila confeccionada para o Laboratório KastlerBrossel, 2001. 
[23] L. Mandel, E. Wolf, Optical coherence and quantum optics, Cambridge University Press, 1995.

[24] S. T. Thornton e J. B. Marion, Classical Dynamics of particles and systems, 2. ed, Thomson Brooks/Cole, 2004.

[25] H. M. Nussenzveig, Curso de Física Básica 1-Mecânica, 3. ed, São Paulo, Editora Edgard Blücher Ltda, 2000.

[26] D.F. Walls e G. J. Milburn, Quantum Optics, Springer-Verlag, 1994.

[27] B. James, Probabilidade: um curso em nível intermediário, Projeto Euclides- Associação Instituto Nacional de Matemática Pura e Aplicada (IMPA), 2006.

[28] F. A. S. Barbosa, A. S. Coelho, K. N. Cassemiro, P. Nussenzveig, C. Fabre, A. S. Villar and M. Martinelli, Quantum state reconstruction of spectral field modes: Homodyne and resonator detection schemes, Physical Review A 88, 052113 (2013).

[29] F. A. S. Barbosa, A. S. Coelho, K. N. Cassemiro, P. Nussenzveig, C. Fabre, M. Martinelli and A. S. Villar, Beyond Spectral Homodyne Detection: Complete Quantum Measurement of Spectral Modes of Light, Phys. Rev. Lett. 111, 200402 (2013).

[30] A. V. Oppenheim e A. S. Willsky com S. Hamid, Sinais e Sistemas, tradução: Daniel Vieira, Rogério Bettoni, revisão técnica: Márcio Eisencraft, Maria D. Miranda, 2. ed, São Paulo, Pearson Prentice Hall, 2010.

[31] G. B. Arfken e H. J. Weber, Mathematical Methods for Physicists, 6. ed, Elsevier Academic Press, 2005.

[32] A. Peres, How the no-cloning theorem got its name, Fortschritte der Physik 51(45): 458-461(2002).

[33] A. Furusawa, P. Van Loock, Quantum teleportation and entanglementA hybrid approach to optical quantum information processing, WileyVCH Verlag GmbH and Co. KGaA (2011).

[34] C.H. Bennett, G. Brassard, C. Crépeau, R. Jozsa, A. Peres and W. $\mathrm{K}$. Wootters, Teleporting an unknown quantum state via dual classical and Einstein-Podolsky-Rosen channels., Physical Review Letters 70, 13 (1993). 
[35] N. Herbert, FLASH-A superluminal communicator based upon a new kind of quantum measurement, Foundations of Physics 12, 12 (1982).

[36] W. K. Wootters, W. H. Zurek, A single quantum cannot be cloned. Nature 299, 802 (1982).

[37] D. Dieks, Communication by EPR devices Phys. Lett. A 92, 271 (1982).

[38] Michael A. Nielsen, Isaac L. Chuang, Quantum Computation and Quantum Information, Springer-Verlag (2000).

[39] D. Bouwmeester, Jian-Wei Pan, K. Mattle, M. Eibl, H. Weinfurter and A. Zeilinger, Experimental quantum teleportation, Nature 390, 575 (1997).

[40] L. Vaidman, Teleportation of quantum states, Physical Review A 49, 1473 (1994).

[41] N. Takei, T. Aoki, S. Koike, K. Yoshino, K. Wakui, H. Yonezawa, T. Hiraoka, J. Mizuno, M. Takeoka, M. Ban, and A. Furusawa, Experimental demonstration of quantum teleportation of a squeezed state, Physical Review A 72, 042304 (2005).

[42] J. Pan, D. Bouwmeester, H. Weinfurter and A. Zeilinger, Experimental Entanglement Swapping: Entangling Photons That Never Interacted, Physical Review Letters 80, 3891 (1998).

[43] N. Takei, H. Yonezawa, T. Aoki, and A. Furusawa, High-Fidelity Teleportation beyond the No-Cloning Limit and Entanglement Swapping for Continuous Variables, Physical Review Letters 94, 220502 (2005).

[44] S. Takeda, T. Mizuta, M. Fuwa, P. Van Loock and A. Furusawa, Experimental Demonstration of Quantum Teleportation of Broadband Squeezing, Physical Review Letters 99, 110503 (2007).

[45] S. Takeda, T. Mizuta, M. Fuwa, P. Van Loock and A. Furusawa, Deterministic quantum teleportation of photonic quantum bits by a hybrid technique, Nature 500, 315 (2013).

[46] I. Marcikic, H. de Riedmatten, W.Tittel, H. Zbinden and N. Gisin Long-distance teleportation of qubits at telecommunication wavelengths, Nature 421, 509 (2003). 
[47] J. Yin, J.Ren, H. Lu, Y. Cao, H. Yong, Y. Wu, C. Liu, S. Liao, F. Zhou, Y. Jiang, X. Cai, P. Xu, G. Pan, J. Jia, Y. Huang, H. Yin, J. Wang, Y. Chen, C. Peng and J. Pan, Quantum teleportation and entanglement distribution over 100-kilometre free-space channels, Nature 488, 185 (2012).

[48] Ma XS1, T. Herbst, T. Scheidl, D. Wang, S. Kropatschek, W. Naylor, B. Wittmann, A. Mech, J. Kofler, E. Anisimova, V. Makarov, T. Jennewein, R. Ursin, A. Zeilinger, Quantum teleportation over 143 kilometres using active feed-forward, Nature 489, 269 (2012).

[49] P. Van Loock, S.L. Braunstein, H. J. Kimble, Broadband teleportation, Physical Review A 62, 022309 (2000).

[50] S.L. Braunstein, Christopher A. Fuchs and H. J. Kimble, Criteria for continuous-variable quantum teleportation, Journal of Modern Optics 47, 267 (2000).

[51] K. Hammerer, M.M. Wolf, E. S. Polzik and J.I. Cirac, Quantum benchmark for storage and transmission of coherent states, Phys. Rev. Lett. 94, 150503 (2005).

[52] S.L. Braunstein, H.J. Kimble, A posteriori teleportation, Nature394, 27 (1998).

[53] S.L. Braunstein, P. Van Loock, Quantum information with continuous variables, Reviews of Modern Physics 77, 513 (2005).

[54] W. P. Bowen, N. Treps, B. C. Buchler, R. Schnabel, T. C. Ralph, H.-A. Bachor, T. Symul, and P. K. Lam, Experimental investigation of continuous-variable quantum teleportation, Physical Review A 67, 032302 (2003).

[55] T. C. Zhang, K. W. Goh, C. W. Chou, P. Lodahl, and H. J. Kimble, Quantum teleportation of light beams, Physical Review A 67, 033802 (2003).

[56] M. Yukawa, H. Benichi and A. Furusawa, High-Fidelity continuousvariables quantum teleportation toward multistep quantum operations, Physical Review A 77, 022314 (2008).

[57] H. J. Kimble, The quantum internet, Nature 453, 1023 (2008).

[58] M. D. Reid e P. D. Drummond, Quantum correlations of phase in nondegenerate parametric oscillation, Phys. Rev. Lett. 60, 2731 (1988). 
[59] L. M. Duan, G. Giedke, J.I. Cirac e P. Zoller, Inseparability criterion for continuous variable systems, Phys. Rev. Lett. 84, 2722 (2000).

[60] J. E. S. César, A. S. Coelho, K. N. Cassemiro, A.S. Villar, M. Lassen, P. Nussenzveig, M. Martinelli, Extra phase noise from thermal fluctuations in nonlinear optical crystals, Physical Review A, 79, 063816 (2009).

[61] Ling-An Wu, H. J. Kimble, J. L. Hall, and Huifa Wu, Generation of Squeezed States by Parametric Down Conversion, Phys. Rev. Lett. 57, 2520 (1986).

[62] T. Golubeva, Yu. Golubev, C. Fabre e N. Treps, Quantum state of an injected TROPO above threshold: purity, Glauber function and photon number distribution, Eur. Phys. J. D. 46, 179 (2008).

[63] P. van Loock e A. Furusawa, Detecting genuine multipartite continuousvariable entanglement, Physical Review A, 67, 052315 (2003).

[64] A. Yariv, P. Yeh, Photonics Optical Eletronics in Modern Communications, Oxford University Press, Cambridge (2007).

[65] Felippe Alexandre Silva Barbosa, Robustez do emaranhamento em variáveis contínuas e fotodetecção de feixes intensos no dominio espectral, Tese de doutorado (Universidade de São Paulo) (2013).

[66] A. S. Villar, M. Martinelli, P. Nussenzveig, Testing the entanglement of intense beams produced by a non-degenerate optical parametric oscillator, Opt. Commun. 242, 551 (2004).

[67] R. W. Boyd, Nonlinear Optics, 3. ed, Academic Press, 2003.

[68] Igor Konieczniak, Teletransporte de informação quântica entre campos de cores distintas, Dissertação de mestrado( Universidade de São Paulo) (2013).

[69] Eric D. Black, An introduction to Pound-Drever-Hall laser frequency stabilization, Am. J. Phys.69 (1) (2001).

[70] Katiúscia Nadyne Cassemiro, Correlações quânticas multicolores no oscilador paramétrico ótico, Tese de doutorado (Universidade de São Paulo) (2008). 
[71] Jônatas Eduardo da Silva César, Excesso de ruído no oscilador paramétrico ótico, Dissertação de mestrado( Universidade de São Paulo) (2008).

[72] Antônio Sales Oliveira Coelho, Emaranhamento tripartite no oscilador paramétrico ótico, Dissertação de mestrado( Universidade de São Paulo) (2009).

[73] V.G.Dmitriev, G.G. Gurzadyan, D.N. Nikogosyan, Handbook of Nonlinear Optical Crystal, 3. ed, Springer, 1999.

[74] B. Willke, N. Uehara, E. K. Gustafson, R. L. Byer, P. J. King, S. U. Seel e R. L. Savage, Jr. Spatial and temporal filtering of a 10-W Nd:YAG laser with a Fabry-Perot ring-cavity premode cleaner, Optics Letters, 23 (1998).

[75] A. Einstein, B. Podolsky, and N. Rosen, Can Quantum-Mechanical Description of Physical Reality Be Considered Complete? Phys. Rev. 47, 777-780 (1935).

[76] Ulf Leonhardt, Essential Quantum Optics from Quantum Measurement to Black Holes, Cambridge University Press, Cambridge, United King$\operatorname{dom}(2010)$.

[77] Celso Jorge Villas-Bôas, Teletransporte e engenharia de estados quânticos, Dissertação de mestrado( Universidade Federal de São Carlos) (2000).

[78] T. Ide, H.F.Hofmann, T. Kobayashi and A. Furusawa, Continuousvariable teleportation of single-photon states, Physical Review A 65, 012313 (2001).

[79] S.L. Braunstein, H.J. Kimble, Teleportation of continuous quantum variables, Physical Review Letters 80, 4 (1998).

[80] R. Simon, Peres-Horodecki separability criterion for continuous variable systems, Phys. Rev. Lett. 84, 2726 (2000).

[81] P. van Loock e S. L. Braunstein, Multipartite entanglement for continuous variables: a quantum teleportation network, Phys. Rev. Lett. 84, 3482 (2000).

[82] T. Debuisschert, A. Sizmann, E. Giacobino e C. Fabre, Type II continuous-wave optical parametric oscillators: oscillation and 
frequency-tuning characteristics, Journal of the Optical Society of America $\mathrm{B}, 10$ (1993). 